University of Louisville ThinkIR: The University of Louisville's Institutional Repository

Electronic Theses and Dissertations

$5-2016$

\title{
Identification of host factors required for Yersinia pestis macrophage intracellular survival and their impact on vacuole maturation, acidification and trafficking.
}

Michael Graylin Connor

University of Louisville

Follow this and additional works at: https://ir.library.louisville.edu/etd

Part of the Bacteriology Commons, Cell Biology Commons, Pathogenic Microbiology Commons, and the Systems Biology Commons

\section{Recommended Citation}

Connor, Michael Graylin, "Identification of host factors required for Yersinia pestis macrophage intracellular survival and their impact on vacuole maturation, acidification and trafficking." (2016). Electronic Theses and Dissertations. Paper 2378.

https://doi.org/10.18297/etd/2378

This Doctoral Dissertation is brought to you for free and open access by ThinkIR: The University of Louisville's Institutional Repository. It has been accepted for inclusion in Electronic Theses and Dissertations by an authorized administrator of ThinkIR: The University of Louisville's Institutional Repository. This title appears here courtesy of the author, who has retained all other copyrights. For more information, please contact thinkir@louisville.edu. 


\title{
IDENTIFICATION OF HOST FACTORS REQUIRED FOR YERSINIA PESTIS MACROPHAGE INTRACELLULAR SURVIVAL AND THEIR IMPACT ON VACUOLE MATURATION, ACIDIFICATION AND TRAFFICKING
}

\author{
Dissertation by: \\ Michael Graylin Connor \\ B.S., Lees-McRae College, 2010 \\ M.S., University of Louisville, 2012 \\ A Dissertation \\ Submitted to the Faculty of the \\ School of Medicine of the University of Louisville \\ In partial fulfillment of the requirements for the degree of: \\ Doctor of Philosophy in Microbiology and Immunology \\ Department of Microbiology and Immunology \\ University of Louisville School of Medicine \\ Louisville, KY
}

May 2016 
Copyright 2016 by Michael Graylin Connor

All rights reserved 



\title{
IDENTIFICATION OF HOST FACTORS REQUIRED FOR YERSINIA PESTIS MACROPHAGE INTRACELLULAR SURVIVAL AND THEIR IMPACT ON VACUOLE MATURATION, ACIDIFICATION AND TRAFFICKING
}

\author{
Dissertation by: \\ Michael Graylin Connor \\ B.S., Lees-McRae College, 2010 \\ M.S., University of Louisville, 2012
}

A Dissertation Approved on

April 1, 2016

by the following Dissertation Committee:

\begin{tabular}{c}
\hline $\begin{array}{c}\text { Dissertation Director } \\
\text { Matthew B. Lawrenz }\end{array}$ \\
\hline Donghoon Chung \\
\hline Yousef Abu-kwaik \\
\hline Micah Worley \\
\hline Jill Suttles
\end{tabular}

Jill Suttles 


\section{DEDICATION}

Over my life I have been challenged by my family, friends and coaches to pursue the highest caliber of competition and life pursuits. From these experiences I have learned dedication, work ethic and perseverance. Of the greatest experiences, I was taught failure is but a measure of will, work, and resilience. The works to follow are a testament to those people throughout my life that have witnessed both my triumphs and failures with equal passion to inspire me to accomplish my dreams. 


\section{ACKNOWLEDGEMENTS}

First off, I would like to thank Matt Lawrenz for allowing me the opportunity to study plague in his lab, and dealing with my excited personality. I genuinely appreciate Matt's mentorship and the opportunities he has given me to develop as a scientist. Matt was not only an excellent scientific mentor, but a great friend over the years and I am humbled to consider him as such. The Lawrenz lab members Jarrod Pennington, Tiva VanCleave, and Amanda Pulsifer are great friends and my adoptive family. Finally, Marlene Steffen for making the research resources at my disposal work; even at odd hours and in the snow.

I would like to thank my committee members, especially Hoon and Yousef for their conversations. I also would like to thank Tom Mitchell, Carolyn Casella, Jim Graham, Rob Hancock, Chris Price, Jeremy Camp, Jared and Cortney "Mushill", Nikki Warner, and Maria Gutierrez. Your friendships, conversations, and time have been some of the greatest experiences of my life.

Finally, I would like to thank my family. Through everyone's endless patience, support and the occasional push, I have accomplished what began as merely a thought fifteen years ago. 


\section{ABSTRACT \\ YERSINIA PESTIS EXPLOITS HOST ENDOCYTIC RECYCLING AND VESICULAR TRAFFICKING FOR INTRACELLULAR SURVIVAL}

Michael G. Connor

April 1, 2016

$Y$. pestis is a facultative intracellular pathogen and the causative agent of plague. This bacterium, while most noted or the Black Death during the European $14^{\text {th }}$ century, is not a historic pathogen but a re-emerging pandemic with both domestic and global impact. Y. pestis is capable of colonizing the macrophage, and actively subverts phagolysosome maturation to establish a replicative niche known as the Yersinia containing vacuole (YCV). The exploited host factors required to support the $\mathrm{YCV}$ are unknown. Here we identified a comprehensive list of host factors required for $Y$. pestis survival through a genome-wide RNAi high-throughput screen. We further identify that avoidance of the phagolysosome requires early recruitment of Rab1b and Rab4a on the YCV. Finally, we show that during intracellular infection Y. pestis sequesters Rab11b to the YCV to stall host cell recycling and support bacterial replication after 8 hours post-infection. These data identify the first host factors required for $Y$. pestis survival within the macrophage, avoidance of phagolysosome maturation, and a novel role for exploiting the host recycling pathway for bacterial replication. 


\section{TABLE OF CONTENTS}

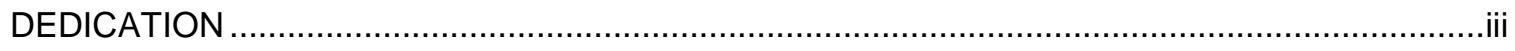

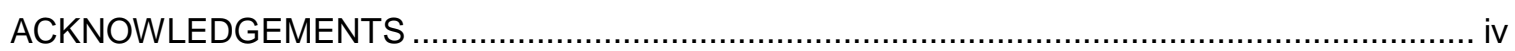

ABSTRACT …

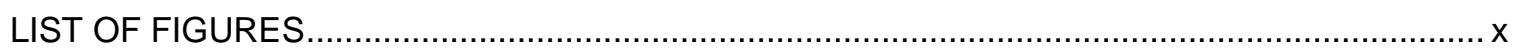

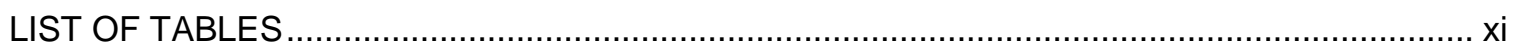

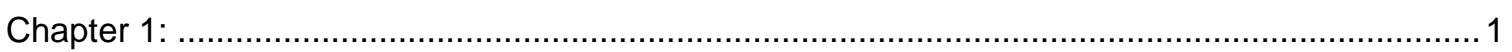

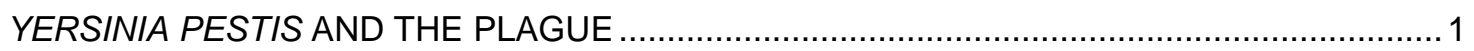

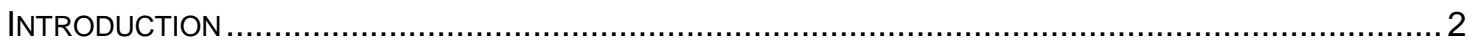

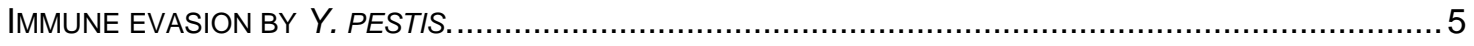

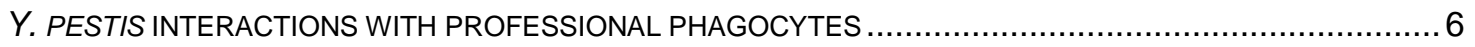

SUBVERSION OF THE PHAGOLYSOSOME BY OTHER PATHOGENS ............................................... 8

Rab GTPases Modulation by Mycobacterium tuberculosis ............................................ 9

Rab GTPase Modulation by Legionella pneumophilia ..................................................... 9

RNAI SCREENS IDENTIFY NOVEL PATHOGENESIS MECHANISMS .............................................. 10

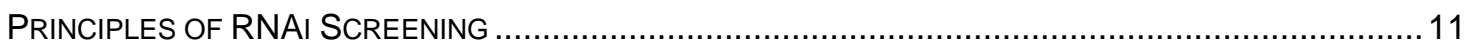

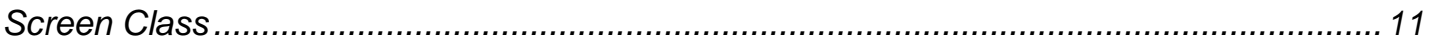

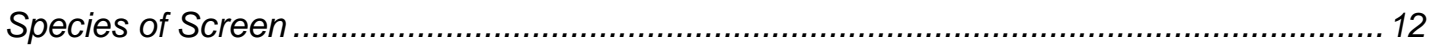

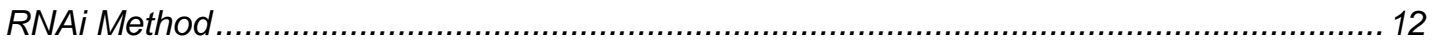

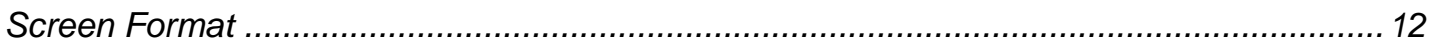

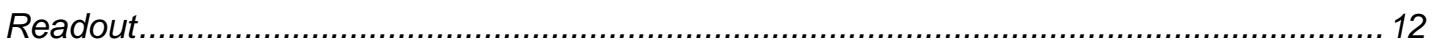

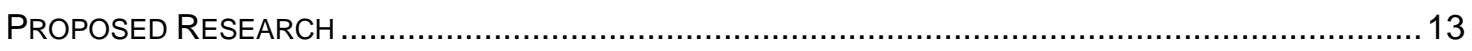

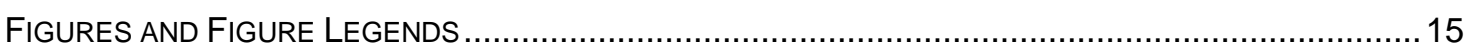

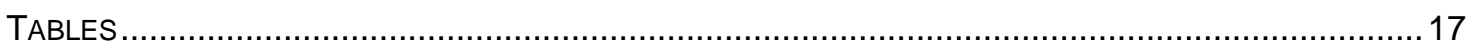

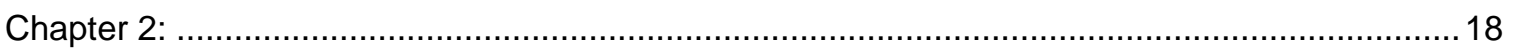

DEVELOPMENT OF BIOLUMINESCENT BIOREPORTERS FOR IN VITRO AND IN VIVO

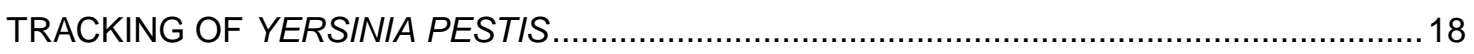

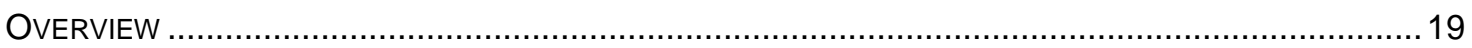

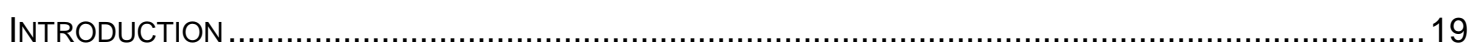

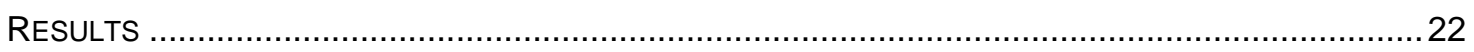

Construction of a chromosomal luciferase reporter system in Y. pestis ...............................22

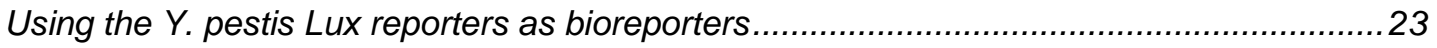

Differentiation between bacterial phenotypes in vitro using Y. pestis Lux bioreporters ........24

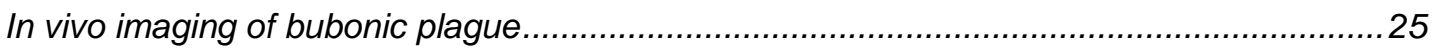

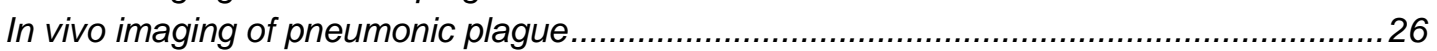

Differences in phenotypes can be detected in vivo using the LuX PcysZK $_{\text {bioreporter ...............26 }}$

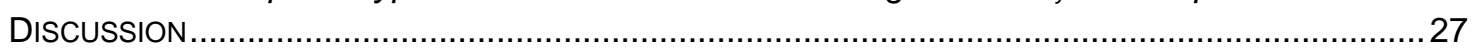

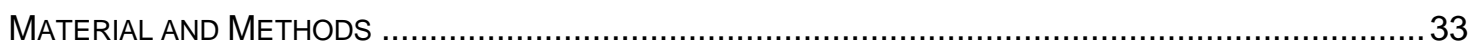

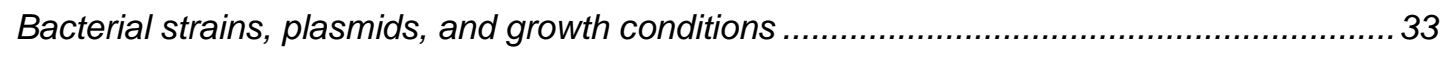

Survival of $Y$. pestis in the presence of antimicrobial compounds ........................................ 34 


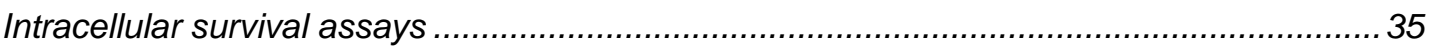

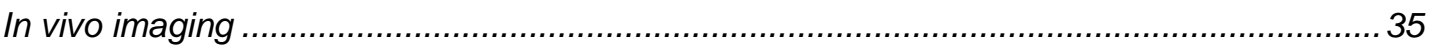

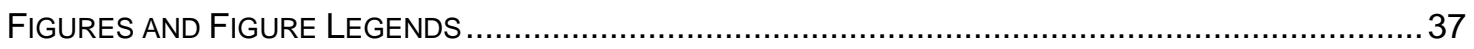

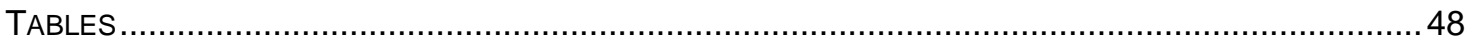

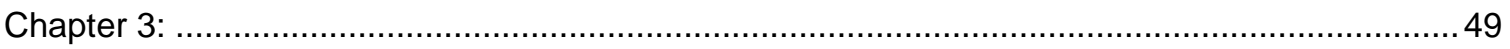

YERSINIA PESTIS REQUIRES HOST RAB1B FOR SURVIVAL IN MACROPHAGES ........... 49

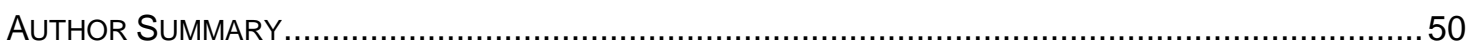

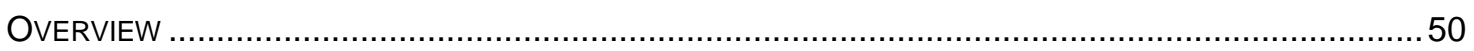

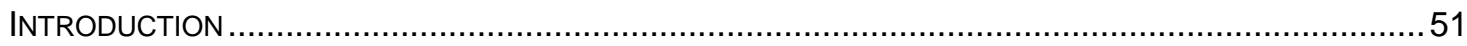

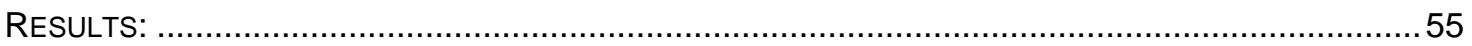

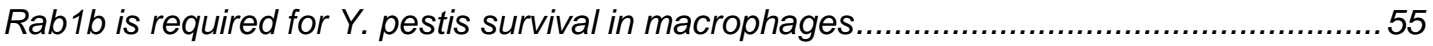

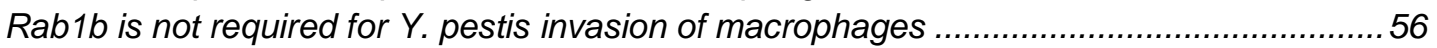

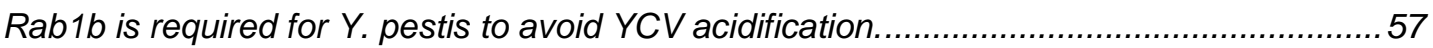

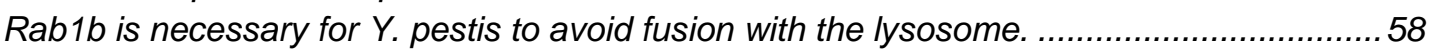

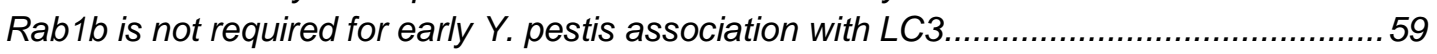

Rab1b is recruited to the YCV during macrophage infection.............................................59

Disruption of the secretory pathway does not alter Y. pestis survival or inhibition of YCV

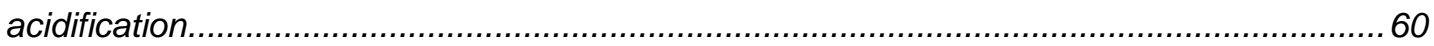

Rab1b inhibition results in increased acidification of the Legionella containing vacuole....... 61

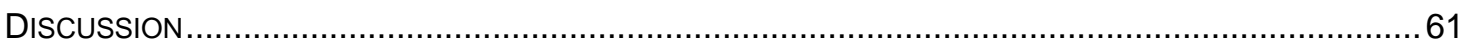

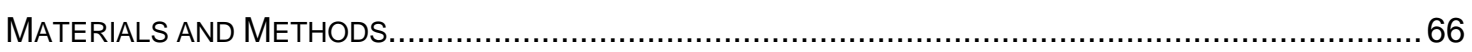

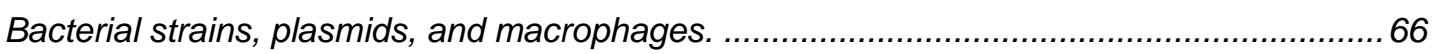

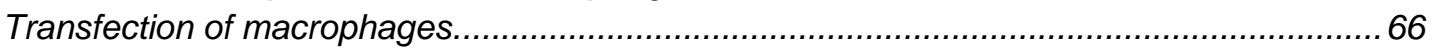

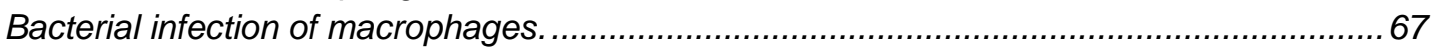

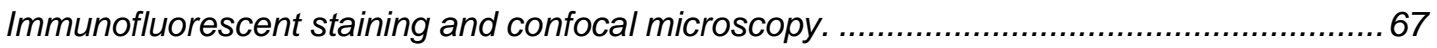

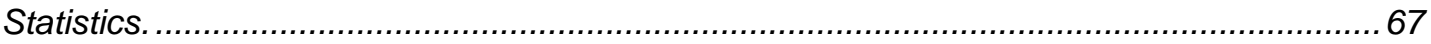

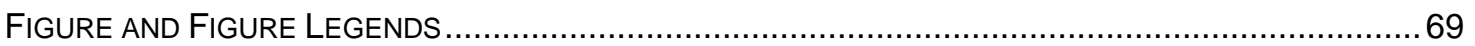

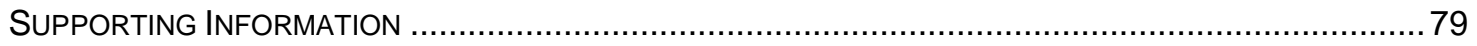

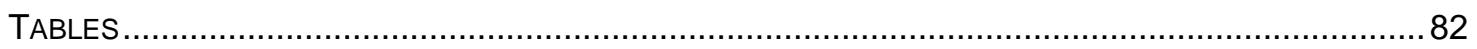

Chapter 4:

\section{GENOME-WIDE RNAI HIGH-THROUGHPUT SCREEN IDENTIFIES THAT YERSINIA} PESTIS EXPLOITS THE HOST ENDOCYTIC RECYCLING PATHWAY FOR

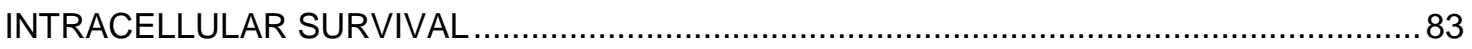

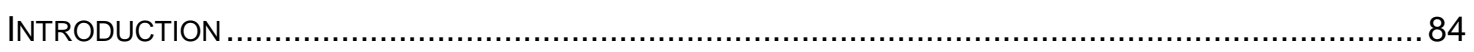

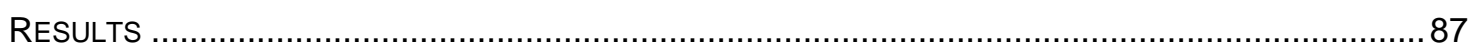

Development of a high throughput assay to monitor the impact of host gene RNAi on Y.

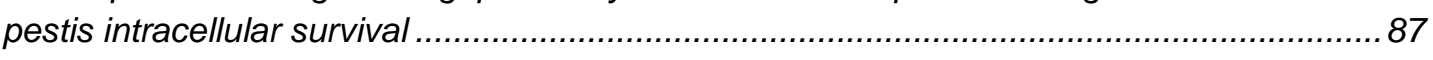

Genome-wide siRNA screen identifies host cell signal transduction, transport and

localization pathways are required for $Y$. pestis intracellular survival................................... 88

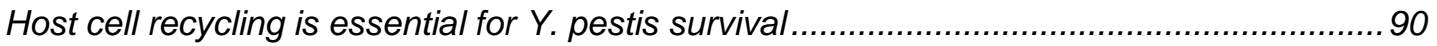

$Y$. pestis requires Rab4a of the recycling pathway to avoid YCV acidification ...................... 91

The Yersinia containing vacuole acquires recycling endosome markers ............................. 92

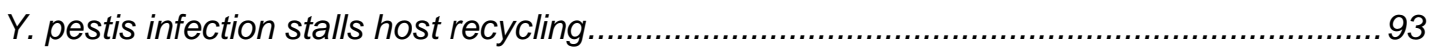

Stalling host cell recycling is required for Y. pestis replication ........................................... 94

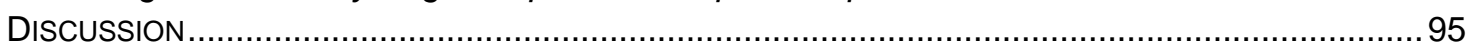

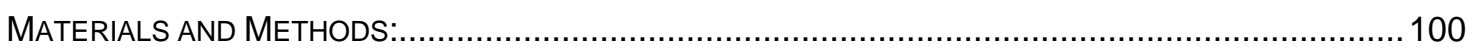


Bacterial strains, plasmids, siRNA and transfection of macrophages. .............................. 100

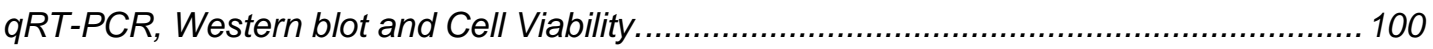

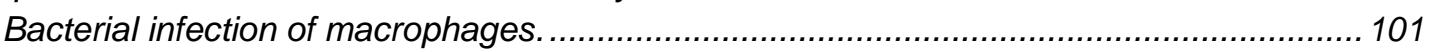

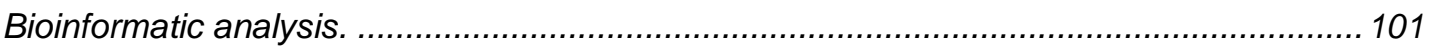

TfR recycling and Acidification assay, Immunofluroescent staining, and confocal microscopy

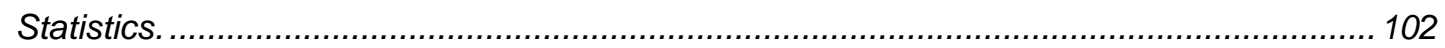

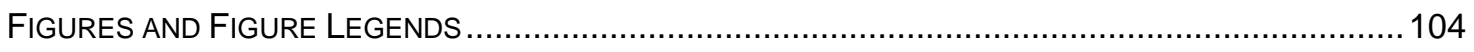

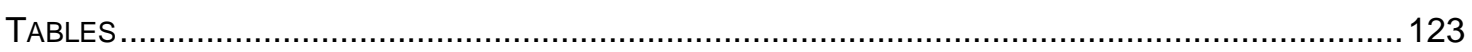

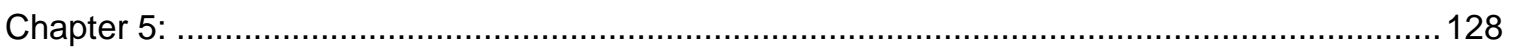

GENOME-WIDE RNAI HIGH-THROUGHPUT SCREEN IDENTIFIES POTENTIAL GENES

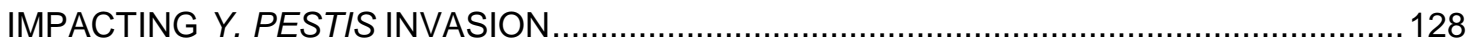

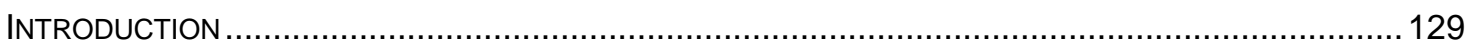

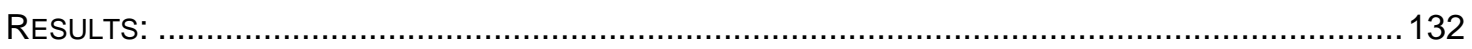

Elevated RLU at 20 min post-infection indicates a defect in Y. pestis invasion. ................. 132

Identifying invasion dataset from the Genome-wide RNAi HTS ....................................... 133

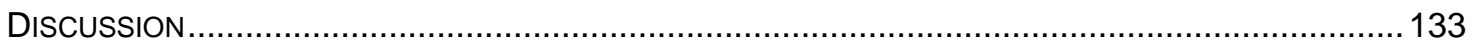

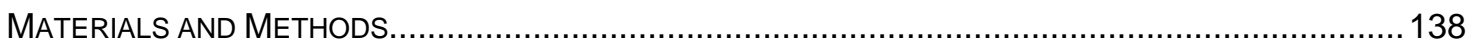

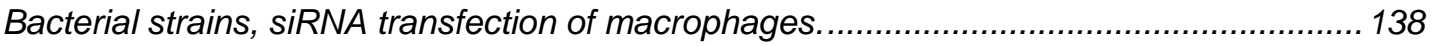

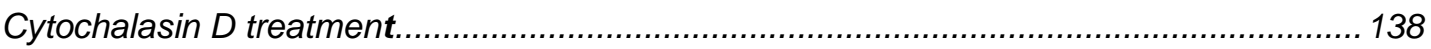

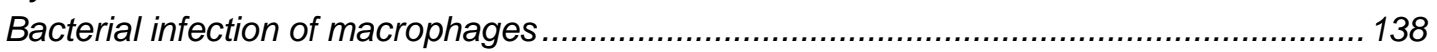

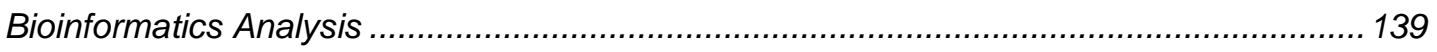

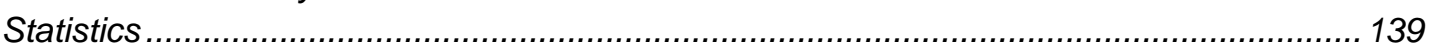

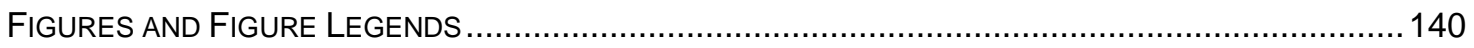

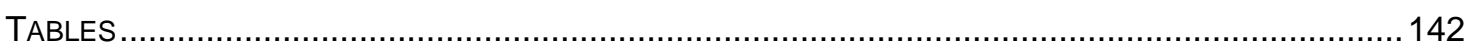

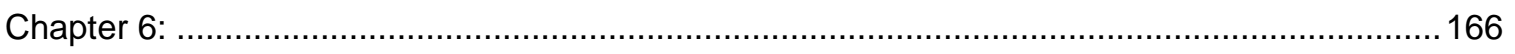

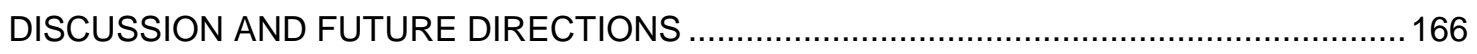

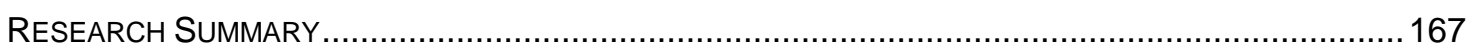

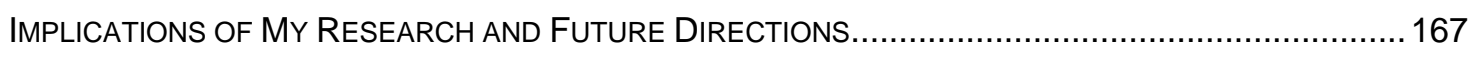

Developing new pathway analysis techniques for RNAi screens through mapping

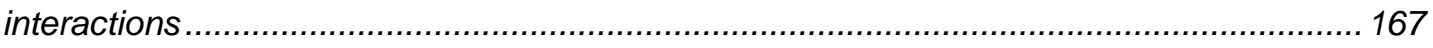

Are Rab1b and Rab4a only required to avoid YCV acidification? .................................... 168

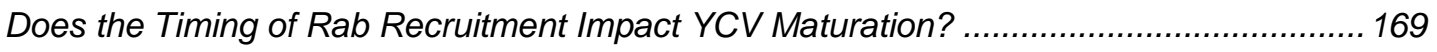

How does Y. pestis recruit Rab GTPases to the YCV? .................................................. 170

How do $Y$. pestis interactions with the host cell recycling pathway contribute to intracellular

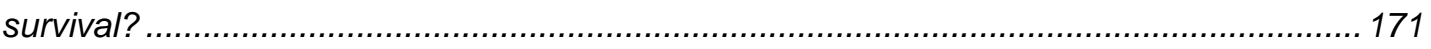

Extending our Rab11b studies suggests a universal mechanism for intracellular pathogens

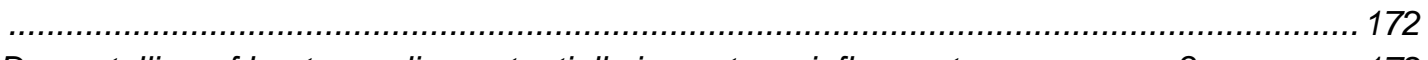

Does stalling of host recycling potentially impact pro-inflammatory responses? ................. 172

Does $Y$. pestis use eukaryotic receptors to target the recycling pathway? ........................174

Is spatial location within the cell important for intracellular survival? ................................. 175

Potential Biological Impact of Y. pestis Low Calcium Response in Macrophages ............... 176

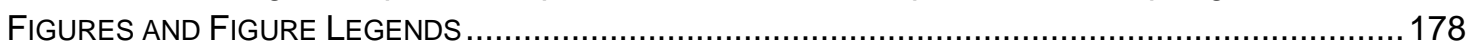

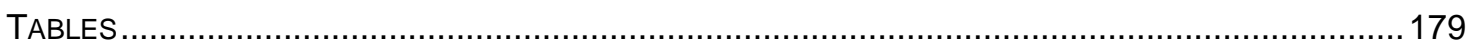

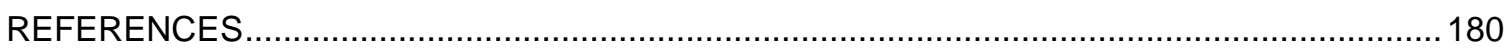




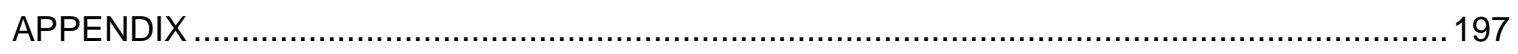

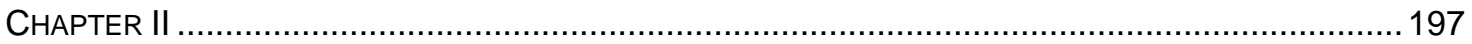

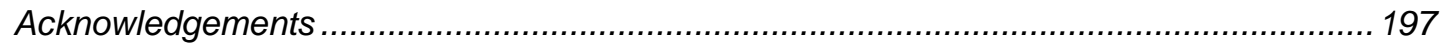

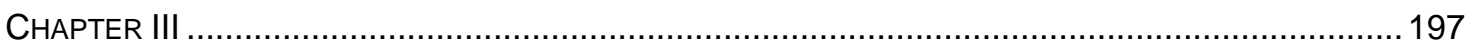

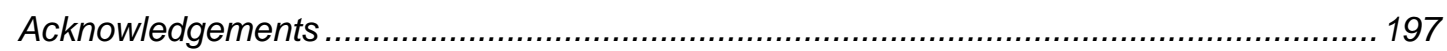

DEPARTMENT OF MICROBIOLOGY AND IMMUNOLOGY CHAIRMAN'S CITATION............198

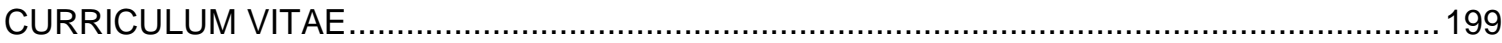




\section{LIST OF FIGURES}

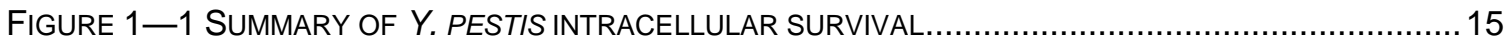

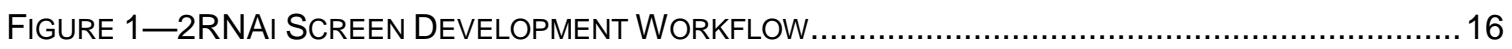

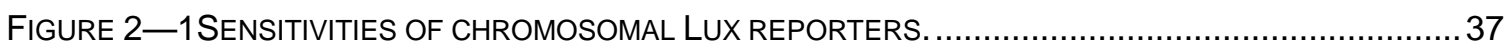

FIGURE 2-2CORRELATION BETWEEN BIOLUMINESCENCE AND BACTERIAL NUMBER ........................... 38

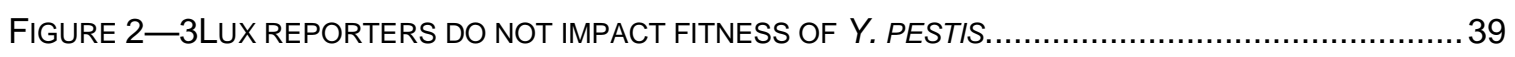

FIGURE 2-4USE OF LUXPTOLC TO MONITOR SURVIVAL OF Y. PESTIS IN THE PRESENCE OF

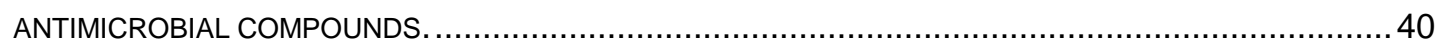

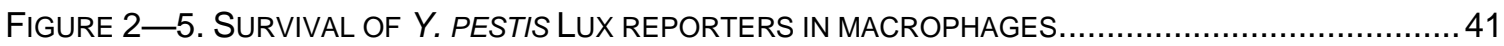

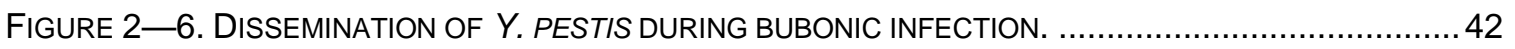

FIGURE 2-7. CONTINUED BIOLUMINESCENCE FROM INOCULATION SITE. ...................................... 44

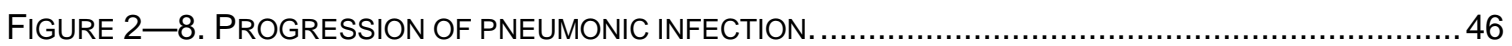

FIGURE 2-9. EXTENDED IMAGING OF ANIMALS INTRANASALLY INFECTED WITH $\triangle$ PLA. ....................... 47

FIGURE 3-1. RAB1B KNOCKDOWN INHIBITS THE SURVIVAL OF Y. PESTIS WITHIN MACROPHAGES. ........ 69

FIGURE 3-2. RAB1B KNOCKDOWN DOES NOT IMPACT Y. PESTIS INVASION OF MACROPHAGES..............71

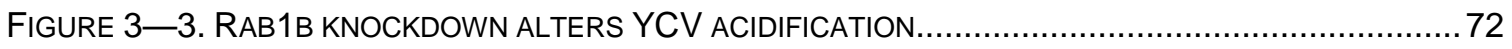

FIGURE 3-4. RAB1B KNOCKDOWN INCREASES YCV ASSOCIATION WITH LAMP1 .............................. 74

FIGURE 3-5. RAB1B KNOCKDOWN DOES NOT AFFECT YCV ASSOCIATION WITH LC3. ........................ 75

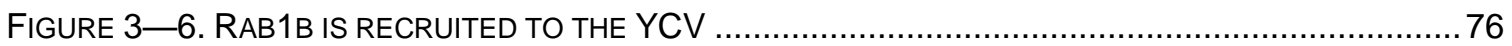

FIGURE 3-7. INHIBITION OF THE SECRETORY PATHWAY DOES NOT INHIBIT Y. PESTIS INTRACELLULAR

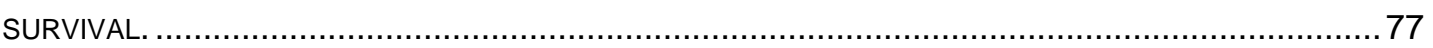

FIGURE 3-8. KNOCKDOWN OF RAB1B INCREASES L. PNEUMOPHILA LCV ACIDIFICATION. .................. 78

FIGURE 3-9. S1 TRANSFECTION WITH RAB1B SIRNA IN MACROPHAGES ........................................... 79

FIGURE 3-10. S2 GROWTH AT $37^{\circ} \mathrm{C}$ DOES NOT ALTER INTRACELLULAR SURVIVAL OF Y. PESTIS. ......... 80

FIGURE 3-11. S3 RAB1B KNOCKDOWN INHIBITS THE SURVIVAL OF ENTERIC YERSINIA WITHIN

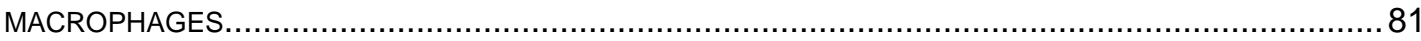

FIGURE 4-1. RNAI-BASED ASSAY TO IDENTIFY HOST FACTORS REQUIRED FOR Y. PESTIS INTRACELLULAR

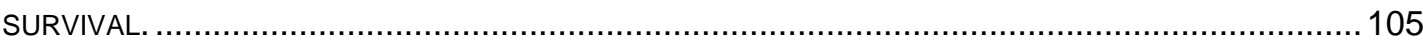

FIGURE 4-2. IDENTIFICATION OF HOST FACTORS REQUIRED FOR Y. PESTIS INTRACELLULAR SURVIVAL. 106

FIGURE 4-3. CLUEGO CLUSTERING OF VALIDATED GENES IMPACTING Y. PESTIS INTRACELLULAR

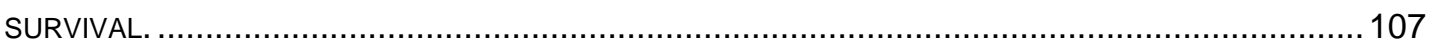

FIGURE 4-4. IDENTIFICATION OF RAB GTPASE INTERACTIONS WITHIN PRIMARY SCREEN HITS............ 109

FIGURE 4-5. HOST RECYCLING INHIBITS Y. PESTIS INTRACELLULAR SURVIVAL ................................111

FIGURE 4-6. RNAI INHIBITION OF RAB4A AND RAB11B DOES NOT IMPACT THE PHAGOLYSOSOME .......112

FIGURE 4-7. RAB4A OF THE RECYCLING PATHWAY IS ESSENTIAL FOR Y. PESTIS TO AVOID THE PHAGOLYSOSOME 114

FIGURE 4-8. Y. PESTIS ACQUIRES RECYCLING ENDOSOME MARKERS RAB4A AND RAB11B.................115

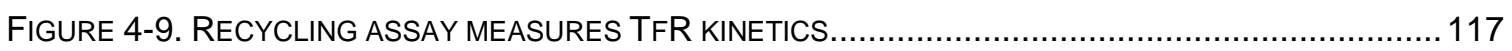

FIGURE 4-10. Y. PESTIS INFECTION STALLS HOST CELL RECYCLING.............................................. 119

FIGURE 4-11. ANT-TFR ANTIBODY DOES NOT IMPACT Y. PESTIS INTRACELLULAR GROWTH.................120

FIGURE 4-12. STALLING HOST CELL RECYCLING IS REQUIRED FOR Y. PESTIS REPLICATION................. 122

FIGURE 5-1. ELEVATED RLU IS CONSISTENT AND PROMOTER INDEPENDENT. ................................... 140

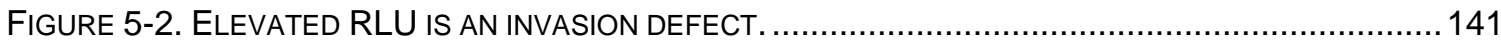

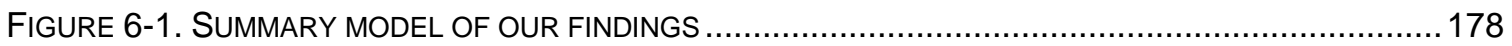




\section{LIST OF TABLES}

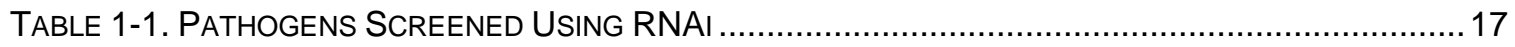

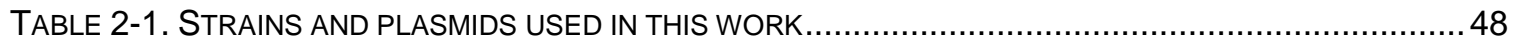

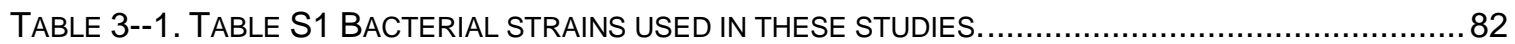

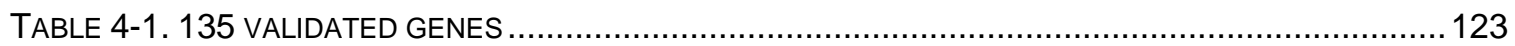

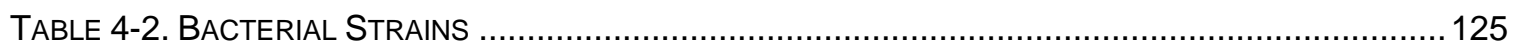

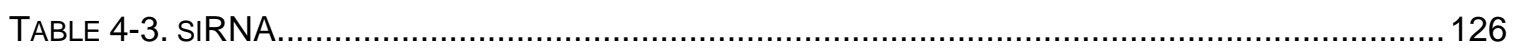

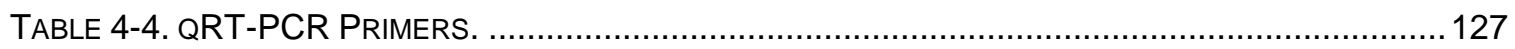

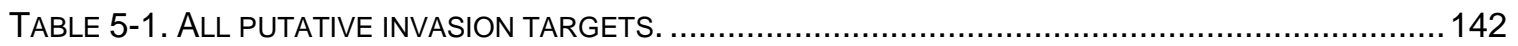

TABLE 5-2. PANTHER IDENTIFIED RECEPTOR/RECEPTOR ACTIVITY HITS. .................................... 163

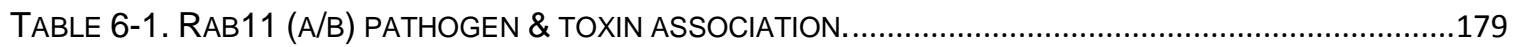


CHAPTER 1:

YERSINIA PESTIS AND THE PLAGUE 
$\underline{\text { Introduction }}$

Yersinia pestis is the etiological agent of the disease known as plague. Y. pestis has caused three major plague pandemics throughout human history. The three pandemics are the "Justinian Plague", the "Black Death", and the "Modern Pandemic". Of the three, the most noted is the Black Death of the $14^{\text {th }}$ century. This outbreak killed one third of the European population, and took over 100 years for the population to recover [1-3]. Y. pestis is a zoonotic pathogen, cycling between the rodent (reservoir host) and the flea transmission vector [1]. The bacterium is endemic throughout rodent populations around the world, including the western United States [4, 5]. Incidence of human plague has been increasing, suggesting that plague is a re-emerging pathogen, and not a historic disease [5, 6]. Y. pestis has been used as a biological weapon and is considered by the U.S. federal government as a high risk for future use as a bioterrorism agent ( $Y$. pestis is a Tier 1 Category A Select Agent) [7].

Human plague manifests in one of three forms: bubonic, septicemic, or pneumonic plague [811]. Bubonic plague is the most common and naturally occurring form of disease. During bubonic plague, bacteria are transmitted to humans through an infectious bite from a flea. From the site of inoculation $Y$. pestis traffics to the draining lymph node. Here the bacteria colonize and replicate to high titer. Infected lymph nodes swell into the hallmark "bubo", a fist-sized lump that resembles a cyst [1]. Bubonic plague is $40-60 \%$ lethal between $5-10$ days post exposure without antibiotic treatment. Untreated bubonic plague can progress to septicemic plague as the bacteria disseminate throughout the host lymphatics and enter the bloodstream [12, 13]. During a septicemic infection the bacteria rapidly spread throughout the host colonizing the spleen, liver and lungs [11]. Lung colonization results in development of pneumonic plague [1], where the bacteria can be transmitted person to person via aerosolized bacteria. Person to person transmission results in primary pneumonic plague and death can occur within 72 hours. Pneumonic and septicemic plagues are $100 \%$ lethal at the onset of symptoms $[1,14]$. In all three forms of disease the minimum infectious dose is believed to be $1-100$ bacteria $[6,15]$.

The genus Yersinia includes Y. pestis, Y. pseudotuberculosis and Y. enterocolitica [16-19]. In the genus, $Y$. pestis is the only species that causes an acute highly fatal infection in the absence 
of timely diagnosis and treatment $[14,20]$. Out of the three species $Y$. pestis is the only vectorborne (flea) pathogen $[1,19]$. Y. pestis began its pathogenic evolution from Y. pseudotuberculosis through the acquisition of two additional plasmids (pMT1 \& pPCP) and genomic rearrangement approximately 1,000 to 20,000 years ago $[16,18]$. There are three biovars of $Y$. pestis, Antiqua, Orientalis and Medievalis $[1,19]$. Of these biovars only Orientalis strains are widespread. Each biovar was once believed to represent pandemic outbreaks of plague throughout human history; with Antiqua representing the Justinian Plague, Medievalis the Black Death (14 ${ }^{\text {th }}$ century), and Orientalis the current pandemic - which began in China [21]. However, given the recent sequence data from victims of the Black Death, and Justinian plagues show that an Orientalis strain is the culprit of all plague outbreaks throughout human history [21-23]. The Y. pestis strain "Colorado 92" (CO92) is a clinical isolate representing the Orientalis biovar [9].

By comparing the genomic sequences of $Y$. pestis across the genus, it was determined that the bacterium is currently undergoing genome reduction and the evolutionary niche it now occupies is potentially filled $[16,18]$. The genome of $Y$. pestis has exchanged genes for various enteric adhesion, invasion and dissemination effectors in favor of genes to maintain transmission between the flea and mammal, such as plasminogen activator (pla) and murine toxin (ymt) $[1,16,18,19]$. However, Y. pestis still maintains the pCD1 encoded Ysc type three secretion system (T3SS), which is conserved across the genus $[1,16,18,19]$. Two of the major genes lost in $Y$. pestis are $\operatorname{yad} A$ and invasin. The $y a d A$ gene is an adhesion that interacts with the extracellular matrix to promote attachment of $Y$. pseudotuberculosis and Y. enterocolitica to the host cell [24-26]. Invasin is a highly efficient molecule that binds $\beta 1$-intergrins to facilitate uptake of both $Y$. pseudotuberculosis and $Y$. enterocolitica by the host through "outside-in" signaling [26-29]. Even though $Y$. pestis lost these conserved factors the bacterium acquired additional virulence factors that contribute heavily to the organism's life cycle. To highlight this, the Plasminogen activator, pla, is protease that is capable of cleaving C3 complement and degrading T3SS effector Yersinia outer membrane proteins (Yops). These functions allow for Y. pestis dissemination throughout the host, and dampening of Yop activation of innate immune responses [30-34]. Pla is encoded on pPCP $[1,16,18,19,35,36]$. The Murine toxin, $y m t$, is required for transmission from the flea vector to in 
mice, and resides on pMT1 $[1,16,18,19,37,38]$. Mutants of $y m t$ result in a 10-fold attenuation in $\mathrm{LD}_{50}$ when mice are intraperitoneally challenged [39-42]. Together, this highlights that $Y$. pestis is a young bacterium of $Y$. pseudotuberculosis descent that is undergoing genomic flux to further streamline its genome to adapt to both the flea and mammalian host.

Plague is not a historical pathogen, but rather a re-emerging disease of great health concern domestically and globally. Globally, plague naturally occurs in the rodent population on every inhabited continent except Australia [5]. The World Health Organization estimates $~ 2,000$ to 4,000 cases of human plague occur annually $[5,6,14]$. A recent review by Butler T et al., 2013, ranked the top twelve countries by human plague cases; China and the United States are seven and eleven, respectively [5]. In both cases the countries have high populations, modern health care, and extensive urbanization. The highly developed nature of these countries highlights the potential for a devastating plague outbreak, and stresses the necessity to understand early pathogenesis to enhance our preventative measures.

To date, there is no Federal Drug Administration (FDA) approved vaccine for Y. pestis [5, $6,14,19,20,43]$. There are three vaccine candidates that target LcrV, the tip of the pCD1 encoded type-three secretion system, and/or the F1 capsule, encoded by caf1 on the PMT1 plasmid. The first two vaccines are subunit vaccines using F1 and LcrV independently and the third is a recombinant fusion protein of $\mathrm{rF} 1 / \mathrm{LcrV}[19,20,43,44]$. These vaccine candidates are currently being comprehensively tested for their efficacy in both bubonic and pneumonic plague in multiple mammalian models. While these vaccines have previously been tested in various animal models, the robustness of lasting $T$ and $B$ cell responses remain undetermined (for review see $[20,43,44]$ ). Vaccine development is slow, in part, because of the $Y$. pestis life cycle. A major concern with current vaccines is isolation of $F 1$ and $\mathrm{pCD} 1$ negative strains naturally form the environment $[1,19$, 43]. These strains spontaneously lose the capsule and the T3SS. Additionally, capsule negative strains still remain lethal in the mammalian host $[1,19]$.

Y. pestis is naturally maintained through reoccurring transmission cycles between the flea vector and rodents $[1,45]$. Humans become infected with $Y$. pestis by their contact with infected 
animals and are accidental hosts in the bacterium's life cycle [1, 8, 9, 11]. Human plague progression is rapid, between 3-10 days, and outpaces the adaptive immune response (see reviews $[1,5,13,14])$. The swift pathogenesis is a hallmark of plague and highlights the critical role innate immunity plays in controlling disease. The natural progression of disease begins with the inoculation of the bacteria from an infected flea bite into the sub-dermis of the host $[1,42,46$ 48]. From this initial site of infection, $Y$. pestis disseminates to the draining lymph node and throughout the lymphatic system [49]. During this process, Y. pestis rapidly suppresses the immune system, turns on several temperature dependent virulence factors, and replicates within the host.

Y. pestis has several well-characterized, mammalian-specific, antiphagocytic virulence factors, like the T3SS, secreted Yop effectors, lipopolysaccharide (LPS), and Caf1 capsule (see review [1]). Interestingly, at initial colonization of the mammalian host these virulence factors are down regulated [1]. Without these antiphagocytic tools $Y$. pestis is easily engulfed by macrophages and neutrophils during initial colonization [49-52]. Neutrophils are readily capable of killing the bacteria, however uptake of $Y$. pestis by a macrophage results in the survival of the bacterium [51, 53-62]. Y. pestis rapidly subverts the innate immune defenses within these cells and establishes a replicative niche termed the Yersinia containing vacuole (YCV) [54, 55, 57, 59].

Immune evasion by $Y$. pestis.

$Y$. pestis uses several virulence determinants to establish infection $[1,33,60,63]$. The best studied of these are the Yersinia outer membrane proteins (Yops), which are secreted by the T3SS $[32,33,63]$. The T3SS forms a channel in the membrane of host cells that the effector Yops (Yop $\mathrm{O}, \mathrm{H}, \mathrm{M}, \mathrm{T}, \mathrm{J}$ and $\mathrm{E})$ are translocated through [33]. Once in the cytosol, the Yops interact with host signaling pathways to prevent phagocytosis and induction of a pro-inflammatory response [32]. $Y$. pestis has been shown to selectively target macrophages, neutrophils and dendritic cells for Yop intoxication $[64,65]$. Bacterial targeting of neutrophils in particular has been demonstrated to be dependent on the bacterial recognition of the host complement receptor 3 [66]. However, the T3SS of $Y$. pestis is temperature dependent and not actively expressed during initial transmission from the flea vector $\left(26^{\circ} \mathrm{C}\right)$ into a mammalian host $\left(37^{\circ} \mathrm{C}\right)$ [1]. 
A second important virulence factor for $Y$. pestis to evade the immune response is LPS $[1,67$, 68]. LPS is normally recognized by TLR4 (toll-like receptor 4), a pathogen recognition receptor, found on phagocytic cells, such as dendritic cells and macrophages. LPS recognition by TLR4 triggers signaling through the TRIF and MyD88 adaptor complexes initiating pro-inflammatory innate immune responses [69-71]. This signaling cascade activates the transcription factor NFk $\beta$ and initiates production and secretion of pro-inflammatory cytokines, such as IL-1 $\beta$ and IL-18 [6971]. However, during plague infection, Y. pestis alters the structure of its LPS and expresses a tetra-acylated form of LPS that does not activate TLR4 $[67,68]$. Furthermore, data from studies with human TLR4 indicate that $Y$. pestis LPS antagonizes TLR4 signaling, actively dampening the pro-inflammatory response, though the exact mechanism remains unclear $[67,68]$. Here again, the LPS modification is temperature dependent and not present at the time of transmission.

Lastly, Y. pestis has an atypical capsule encoded by the caf operon [1,72-74]. This capsule is comprised mainly of helical proteins that are loosely attached to the outer membrane of the bacterium [1, 72-74]. Caf1 is an antiphagocytic virulence factor which prevents phagocytosis by macrophages and monocytes $[75,76]$. However, studies have demonstrated that capsule mutants are no less virulent than their wildtype counterparts, and at best display only a modest increase in $\mathrm{LD}_{50}$ in mice (summary of strain LD 50 table $\left.2[1] ;[39,77]\right)$. Furthermore, caf1 expression is regulated by temperature and active only at $37^{\circ} \mathrm{C}[78,79]$.

All three of these virulence factors are specialized for the mammalian host and required for $Y$. pestis to avoid phagocytosis by innate immune cells. However, they are not expressed in the flea vector, and are temperature regulated (flea $26^{\circ} \mathrm{C}$ to mammal at $37^{\circ} \mathrm{C}$ ) [1]. Because of this $Y$. pestis during colonization from the flea vector is highly susceptible to uptake by innate immune phagocytes.

\section{$\underline{Y . p e s t i s ~ i n t e r a c t i o n s ~ w i t h ~ p r o f e s s i o n a l ~ p h a g o c y t e s ~}$}

During colonization of the mammal Y. pestis is inoculated into the dermis, sub-dermis or directly into the blood stream via a capillary during a flea bite $[1,42,46]$. At the site of infection there is an influx of neutrophils and macrophages [51]. These professional phagocytes are the innate immune 
system's first responders to infection, and are tasked with controlling infection and priming an immune response. At the site of colonization $Y$. pestis interacts with both neutrophils and macrophages. However, neutrophils are better capable of killing the bacteria at this transitional stage in the bacteria's early pathogenesis $[53,58,80,81]$. In contrast, when a macrophage or monocyte phagocytizes $Y$. pestis, the bacterium prevents killing within these cells and is capable of establishing an intracellular niche $[51,54,55,57,59,82,83]$. In further support of this, $Y$. pestis infected rodents and nonhuman primates show bacteria association with macrophages, but to a lesser extent neutrophils from within the same infected host $[49,53,58,84]$.

Y. pestis taken up by macrophages remain within a phagosomal compartment, but the bacteria inhibit the normal maturation of this phagosome to survive within the macrophage $[57,59,60,62$, $63,83,85,86]$. While the mechanisms are not well understood, $Y$. pestis is able to actively inhibit the acidification of this YCV [59]. The YCV does not acidify and the $\mathrm{pH}$ remains between $\mathrm{pH} 6.5-$ 7.5 throughout intracellular survival $[59,87]$. In contrast to live bacteria, paraformaldehyde killed Y. pestis is rapidly degraded in an acidified YCVs $[59,87]$. Studies have colocalized Rab7 and Cathepsin D, markers for stages of phagolysosome maturation, to $\sim 30 \%$ of all nascent $\mathrm{YCVs}$, indicating that the majority of the bacteria are not in a mature phagolysosome [86, 87]. In contrast, Rab5 and EEA1, markers for the early endosome have not been colocalized to the YCV, either due to lack of these markers on the YCV or the biogenesis of the YCV is too rapid. Approximately eight hours post infection, the phagosome is further modified, and expands into a spacious vacuole in which $Y$. pestis is able to replicate [59]. At this point in YCV biogenesis, the spacious vacuole is clearly observed in light and electron micrographs $[55,59,62,86]$. Studies to characterize the spacious YCV have indicated this compartment acquires LC3-II, a marker of autophagosomes, as early as four hours post-infection, and autophagy potentially contributes to the metabolism of the bacteria $[59,88,89]$. Studies by Ligeon et al., using Y. pseudotuberculosis, show the YCV acquires VAMP3 within 30 mins post-infection and is exchanged for VAMP7 within 3 to 24 hours [89]. They further demonstrated VAMP3 and 7 dynamics are required for YCV LC3-II single/double membrane formation [89]. Additional works submit that the YCV is a phagolysosome [55]. However, unpublished data by Bliska and colleagues indicate the spacious phagolysosome potentially retains 
Lamp1, a lysosomal marker, and/or LC3-II [59, 87]. In either case, the spacious phagolysosome or autophagosome have been both identified, but whether $Y$. pestis requires autophagy for replication remains to be determined.

Several in vivo studies indicate that intracellular survival in macrophages is important for colonization of the mammalian host $[52,62,90]$. Ye et al. demonstrated selective depletion of Gr1+ macrophage and dendritic cells in transgenic MaFIA mice lessens the severity of plague infection [52]. St. John and colleagues further highlight the importance of these innate cells, as chemical inhibition impacts the ability of $Y$. pestis to disseminate from the draining lymph node in a Sphingosine-1-phosphate dependent manner [49]. Additionally, macrophages from canines (a species relatively resistant to plague infection) are better equipped to kill intracellular $Y$. pestis than rodent macrophages (the natural reservoir for plague) [62, 91]. Finally, in vivo infection studies with $Y$. pestis phoP mutants, which are defective for intracellular survival, have reported extended times to death for the phoP mutant compared to wildtype $Y$. pestis [90]. These data suggest intracellular survival of $Y$. pestis also contributes to early immune evasion and pathogenesis.

Biogenesis of the YCV highlights the importance of exploited host-pathogen interactions to facilitate survival of $Y$. pestis within the macrophage. The current model of $Y$. pestis intracellular survival within the macrophage based on all peer-reviewed publications is summarized in Fig. 1-1

\section{Subversion of the Phagolysosome by other pathogens}

Phagolysosome maturation is a well-documented cellular process that requires subsequent recruitment and dissociation of Rab GTPases to coordinated vesicle trafficking and ultimately fusion with the lysosome [92-94]. Rab5, Rab7, and Rab9 are key contributors to the phagosome maturation pathway [94-101]. Initially, Rab5 is recruited to the early endosome and is exchanged with Rab7 on the intermediate phagosome [94, 97-101]. From here, Rab7 is required for the recruitment of Rab9, which completes the fusion of the maturating phagosome with the lysosome [94, 98-101]. At this point, the mature phagolysosome has Cathepsin D and vATPase markers and is actively acidifying to $\mathrm{pH} 4.0$ in order to degrade the vacuole contents [95]. Subtle 
disruptions in the recruitment of the Rab proteins can stall or even terminate trafficking of these endocytic vesicles and many intracellular pathogens hijack this process [102, 103].

Vesicular trafficking has become an area with increased research efforts in the context of pathogenesis (for review see [93, 102-104]). Pathogens such as Mycobacterium tuberculosis and Legionella pneumophilia have developed mechanisms to modulate host Rab GTPase machinery and prevent their destruction within the phagolysosome $[93,102,103]$.

\section{$\underline{\text { Rab GTPases Modulation by Mycobacterium tuberculosis }}$}

Mycobacterium tuberculosis (M. tb) is an intracellular pathogen that causes tuberculosis in humans [105]. The bacterium has developed the ability to exploit host trafficking to prevent degradation in the phagolysosome. Studies dissecting the recruitment and retention of host Rab GTPases to the Mycobacteria containing vacuole (MCV) show the bacterium arrests phagolysosome maturation through disruption of the sequence/order of Rab GTPases [93, 106110]. Briefly, once M. tuberculosis is engulfed, the MCV acquires 22 different Rab GTPases [108]. Of these Rabs, differential recruitment of Rab22a and Rab14 to the early endosome stalls the conversion of Rab5 to Rab7, preventing the maturation of the phagolysosome [107, 109, 110]. Further investigation by Seto and colleagues showed Rab7, Rab20, and Rab39 regulated the acidification of the MCV [108]. In contrast, Rab7, Rab20, Rab22b, Rab32, Rab34, Rab38 and Rab43 impacted the recruitment of cathepsin D to these phagosomes [108]. Of note, Seto and colleagues' work highlights speculation that $M$. tuberculosis does not actively target host Rab GTPases, but rather acquires Rab GTPases that normally modulate the process of phagolysosome maturation as a byproduct of how M. tuberculosis is trafficked upon uptake [108]. In support of this, recruitment of Rab22a, a Rab GTPase normally found on recycling endocytic vesicles, is associated and retained on the MCV $[93,106]$.

\section{$\underline{\text { Rab GTPase Modulation by Legionella pneumophilia }}$}

Legionella pneumophilia is a facultative intracellular pathogen and the causative agent of Legionnaire's disease [111, 112]. Legionella has a type 4 secretion system (T4SS) and translocates over 300 effector proteins to modulate the host cell upon engulfment $[111,112]$. The bacterium 
establishes a Legionella-containing vacuole (LCV) within the host cell and remodels this niche into a hospitable environment for replication. One of the best studied host targets for Legionella's bacterial effectors is the host Rab1 GTPase (further detailed in the discussion of Chapter 3). Briefly, L. pneumophilia secretes several effectors that actively modulate the activity of host Rab1 on the LCV [113-126]. This process relies on bacterial modification of the host Rab GTPases' ability to hydrolyze GTP to GDP through cycling of bacterial effectors that, 1) increase Rab1 hydrolysis, 2) AMPylation of Rab1, 3) de-AMPylation of Rab1, and 4) ubiquitination of Rab1 [115-117, 121-129]. The outcome of these modifications on Rab1, results in L. pneumophilia modulation of LCV acidification and blocking of phagolysosome maturation.

\section{$\underline{\text { RNAi Screens Identify Novel Pathogenesis Mechanisms }}$}

To date, there have been multiple genome-wide RNAi high-throughput screens to identify novel targets and pathways exploited by various bacterial and viral pathogens (Table 1-1; [130-142]). This tool has been exceptionally useful for bacterial and viral pathogens that are naturally difficult to perform traditional genetic screens in, such as Coxiella burnetii, West Nile Virus, and Mycobacterium tuberculosis $[131,136,137,139]$. This method is amenable to suitable surrogate infection models and completely rests on the impact of a host gene upon the survival of the pathogen in question. The first genome-wide RNAi screen by Agaisse et al., demonstrated the power of RNAi screen approaches to identify host requirements for survival by Listeria monocytogenes and Mycobacterium fortuitum [141]. Their results compared L. monocytogenes to M. fortuitum to identify individual host components required for both vacuole escape and pathogencontaining vacuole (PCV) maturation. They utilized the advanced tools for Drosophila cell RNAi and network/gene homologies to identify 160 targets specifically impacting L. monocytogenes survival and 91 targets that impacted both organisms' intracellular survival [141]. A major finding from their data was the identification of several Rab GTPases, such as Rab1, 5, 7, and 35, and cellular trafficking components, such as Cop $\beta 1$, that altered survival. These data eventually lead to discoveries of autophagy modulation and the exploitation of host genes to arrest phagosome maturation by various pathogens. While Agaisse et al. pioneered the use of RNAi screens to identify novel genes, it was Kumar et al., who truly established the power of bioinformatic analysis 
of these types of data. Using M. tuberculosis they developed a complex screening system to validate primary screen hits in subsequent secondary and tertiary screens through matching phenotypes and library deconvolution [136]. From their stringent screening process they applied GO ontology and network clustering criteria for the 329 validated hits. Their results demonstrated modular clustering of required host factors for M. tuberculosis that was isolate dependent [136]. Furthermore, they show enrichment for biological networks, such as "Metabolism" and "Immune and Inflammation", that inhibit and regulate survival of $M$. tuberculosis from the same datasets [136]. A highlight from their studies was bioinformatic identification and biological validation of interacting components with the autophagy cascade that dynamically regulated $M$. tuberculosis survival through modulation of IFNy host responses [136]. Overall, genome-wide screening has successfully identified valuable networks exploited by other pathogens. However, development of an RNAi platform requires meticulous optimization and validation before it is implemented in the screening of a pathogen.

\section{Principles of RNAi Screening}

In order to complete an RNAi screen, one must develop and optimize a screening platform to specifically address their hypothesis. Fundamentally, the process of development can be broken down into five parts, 1) class of screen, 2) species of screen or cell type, 3) RNAi method, 4) screen format, and 5) readout (Fig. 1-2; [143, 144]).

\section{$\underline{\text { Screen Class }}$}

In developing a screening platform to address your initial hypothesis one must either select a genome-wide or a functional category class of host targets (Fig. 1-2). A genome-wide screen uses a library comprised of targets from the known coding sequences of the species genome (e.g., Drosophila, Mouse or Human). A functional category class screen is a small subset of host targets and can be a class of protein, such as Kinases or Rab GTPases, or a known pathway, such as JAK/STAT or Ubiquitin. 


\section{Species of Screen}

The next stage is to select the species you will conduct the RNAi in, Drosophila, Mouse or Human (Fig. 1-2). This selection is primarily driven by the ability to achieve knockdown of the target transcript using your chosen RNAi method [144].

\section{$\underline{\text { RNAi Method }}$}

The RNAi delivery method can either be long dsRNA, esiRNA, siRNA, or shRNA (Fig 1$2 ;[143,144])$. Long dsRNA use the host machinery by Dicer to cleave the molecule into pools of siRNA [143]. This method of delivery works best for $C$. elegans and Drosophila, because the dsRNA does not activate the type-I interferon response as it would in mammalian cells $[143,145]$. The synthetic esiRNA and siRNA are developed to mimic the sequences after Dicer processing of the molecule, and are used mainly for mammalian cells and immortal cell lines [143, 146]. Finally, shRNA are designed to mimic the pre-microRNA substrate of Dicer and are expressed via Retroviral or Lentiviral vectors in transduced cells $[143,147]$. The use of shRNA has been shown effective for primary mammalian cells, such as human macrophages [143]. Ultimately, the optimal degree of knockdown to achieve is $70-95 \%$ of the target [143, 144].

\section{Screen Format}

Screening formats are either arrayed or pooled [143]. In an arrayed setup the user screens individual RNAi's with the ability to deconvolute individual results via statistical analysis of their readout [143]. This arrayed approach provides individual data for each of the RNAi's being tested, but can be cost, time and labor prohibitive at the genome-wide level. In a pooled approach, the user combines the individual RNAi's (usually 3-6) and screens them for an effect, then statistically analyzes the results and chooses a subset of targets to further evaluate [143, 144]. A pooled approach lends itself to rapid and cost effective genome-wide screening.

\section{$\underline{\text { Readout }}$}

The final step to platform development is generating a 'readout'. The readout encompasses both the phenotypic reporter system as well as the technique/equipment used to discern the phenotype [143]. The two common phenotypic reporters are fluorescent and 
luminescent. While there are several means to analyze a high-throughput screen (HTS), the most common are either high-content imaging or plate reader based [143, 144, 148]. In both instances, the equipment requires optimization much like the rest of the platform and will briefly be summarized. High-content imaging requires the user to determine the autofocus, image quality minimums, segmentation, threshold, and regions-of-interest $(\mathrm{ROI})$ of the image using software analysis tools [148]. From here, the user can quantify a range of phenotypes based on the amount of fluorescent reporter being imaged. A basic example of this for RNAi pathogen screens is number of bacteria or bacterial total intensity per cell $[133,135,141]$. To date, there has not been a multiplexed approach to determine both pathogen survival and relationship to either an overexpressed protein or antibody stained protein. As for a screen using a plate reader there is less to optimize, as the user is mainly concerned with dynamic range of the reporter's intensity (fluorescent or luminescent) and the separation of controls. Here, the optimization of dynamic range is key to determine the limit of detection for the phenotypic response. As for the separation of controls, the user will account for the phenotypes using a Z' factor per plate, Z' score, or B score $[149,150]$. These statistical approaches are based on the separation of a positive and negative control being $3 \sigma$ (standard deviations) apart. For Z' factor in particular, a score $>0.5$ is highly robust, $<0.5$ to $>0.3$ is acceptable, and $<0.03$ is unacceptable [149].

\section{Proposed Research}

$Y$. pestis is a facultative intracellular pathogen capable of surviving within macrophages. Macrophages are innate immune cells normally capable of killing bacteria, however increasing data show $Y$. pestis prevents this process and survives within these host cells. Survival within macrophages is a potential protective niche for $Y$. pestis during transition from the flea $\left(26^{\circ} \mathrm{C}\right)$ to mammalian $\left(37^{\circ} \mathrm{C}\right)$ host. During this transition the bacteria is not expressing temperature dependent mammalian specific virulence factors, such as the T3SS, and is easily killed by neutrophils. $Y$. pestis $\triangle p h o P$ mutants are defective for intracellular survival and exhibit a higher $\mathrm{LD}_{50}$ with an extended time to death [56, 83, 90, 151]. The role of macrophages during pathogenesis is further emphasized by findings that $Y$. pestis predominately associates with 
macrophages and not neutrophils in infected rodents and nonhuman primates $[49,53,58,84]$. Depletion of macrophages prior to infection with Y. pestis dampens bacterial burden [52].

Y. pestis survives within macrophages in a phagosomal compartment known as the YCV. Y. pestis actively subverts the phagolysosome maturation by inhibiting acidification of the YCV. After eight hours post-infection, the YCV expands in conjunction with bacterial replication $[54,55$, $57,59,61,62,152,153]$. While the fate of $Y$. pestis within the macrophage is known, the mechanisms exploited by $Y$. pestis to inhibit phagolysosome maturation and establish the YCV have not been defined.

The use of genome-wide RNAi screens for comprehensive identification of required factors exploited by various intracellular pathogens for survival suggests the successful adaptation of this technology to $Y$. pestis. With this, we hypothesize that $Y$. pestis actively exploits host pathways/factors to avoid YCV acidification and support YCV biogenesis. To address this, we:

1) Developed a bioluminescent system capable of monitoring bacterial burden within macrophage cells.

2) Optimized, established, and completed a genome-wide RNAi screen in the biologically relevant murine macrophage cell line (RAW264.7 cells).

3) Performed bioinformatic analysis for identified genes and validation of phenotypes from bioinformatic networks.

4) Identified host factors impacting $Y$. pestis intracellular survival, altering YCV biogenesis and avoidance of the phagolysosome.

The following chapters represent the first steps toward comprehensive identification of required host factors and the mechanisms necessary to support $Y$. pestis survival within the macrophage. 
Figures and Figure Legends

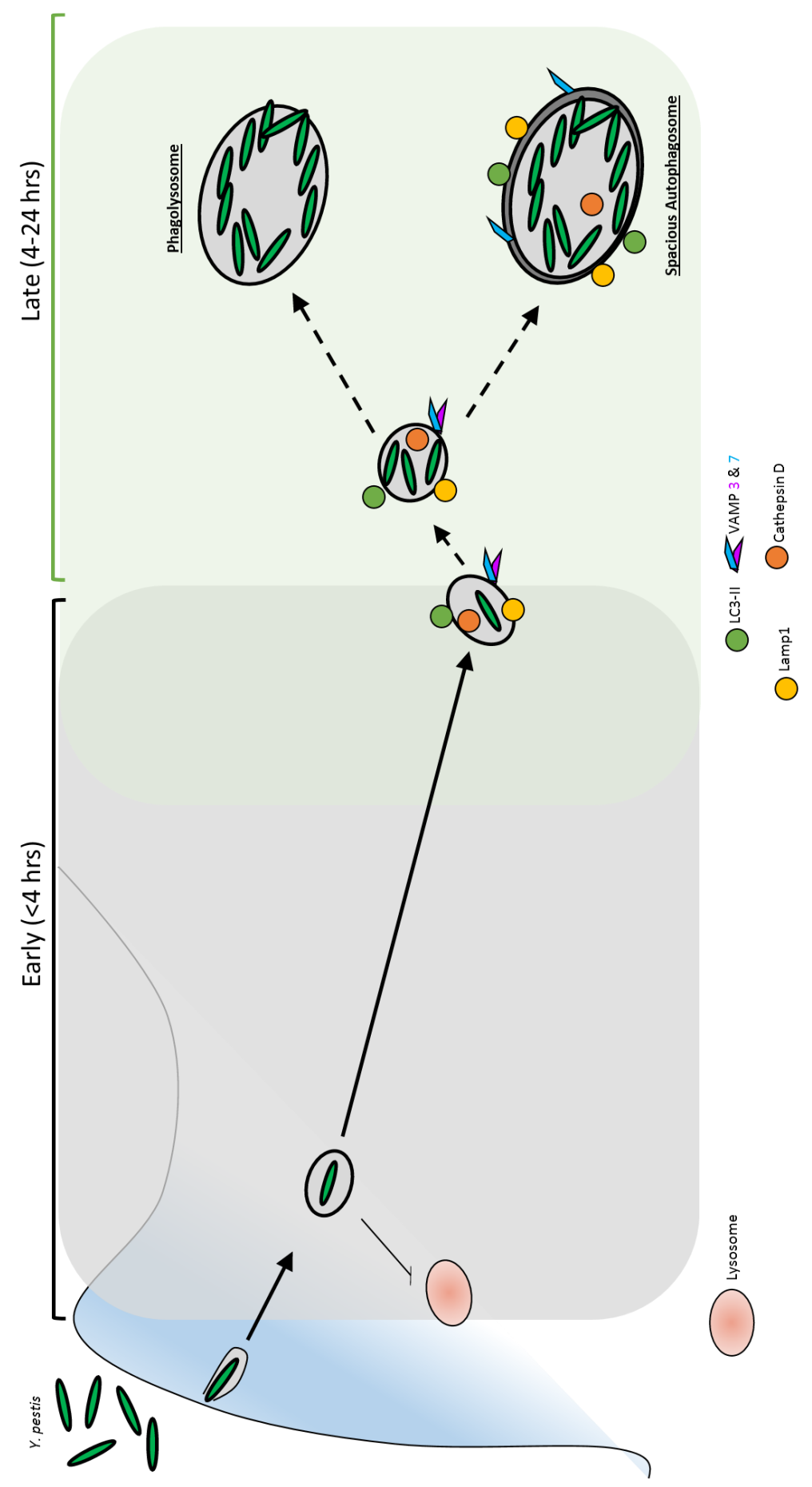

Figure 1-1 Summary of $Y$. pestis intracellular survival 


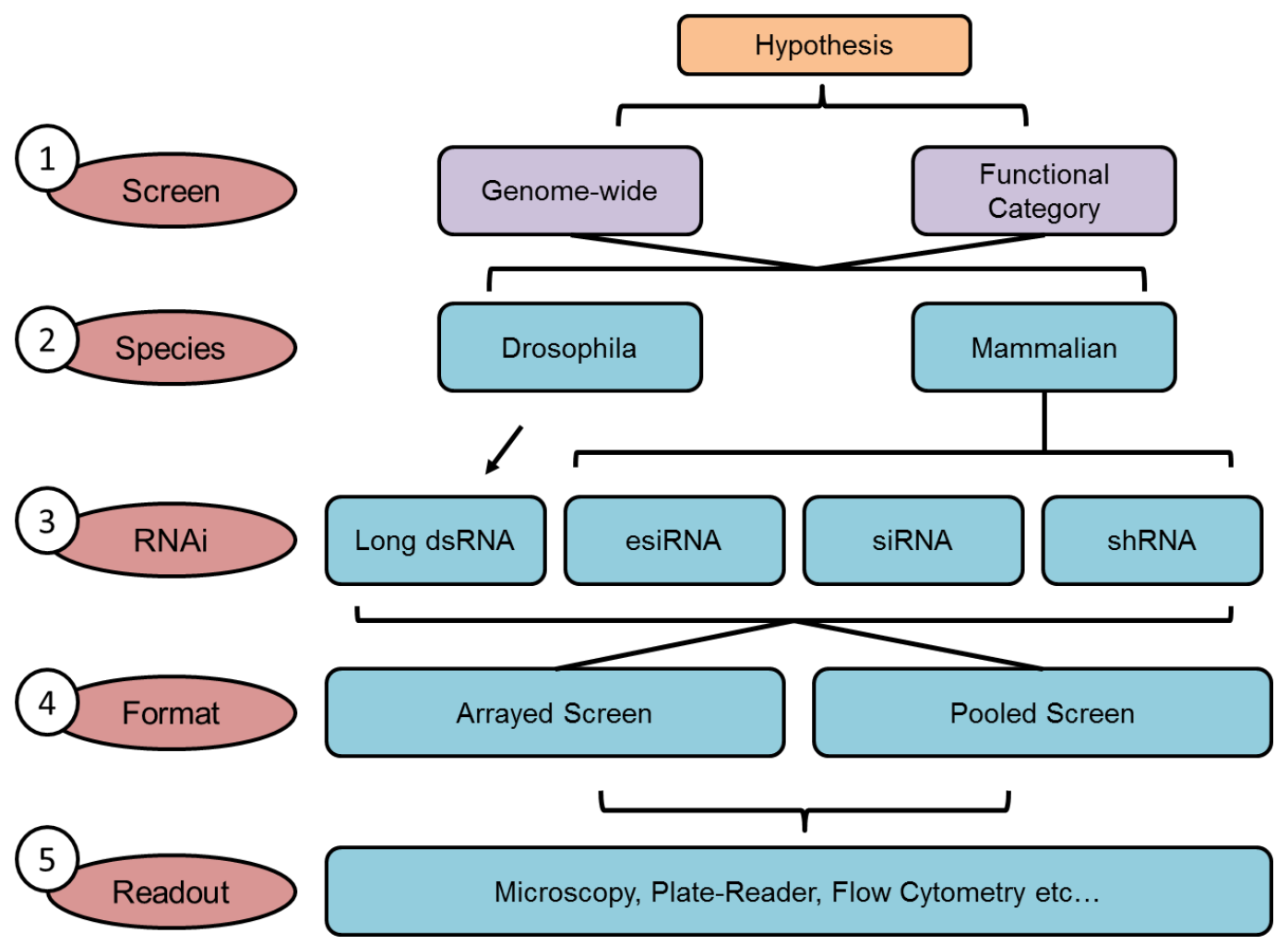

Figure 1-2RNAi Screen Development Workflow 
$\underline{\text { Tables }}$

Table 1-1. Pathogens Screened Using RNAi

\begin{tabular}{|c|c|c|c|}
\hline Pathogen & Cell Type & Year & Reference \\
\hline L. monocytogenes & HeLa CCL-2 & 2015 & $\begin{array}{l}\text { Kuhbacher } \\
{[130]}\end{array}$ \\
\hline C. burnetti & HeLa & 2013 & $\begin{array}{l}\text { McDonough } \\
\text { [131] }\end{array}$ \\
\hline Chikungunya virus & U-2 OS & 2013 & Ooi [132] \\
\hline $\begin{array}{l}\text { Eastern Equine } \\
\text { Encephalitis virus }\end{array}$ & U-2 OS & 2013 & Ooi [132] \\
\hline S. typhmurium & MCF-7 & 2012 & $\begin{array}{l}\text { Thornbroug } \\
\mathrm{h}[133]\end{array}$ \\
\hline F. tularensis & $\begin{array}{l}\text { Human Monocyte-like THP-1; } \\
\text { HMDMs; Human Alveolar } \\
\text { Macrophages }\end{array}$ & 2012 & Zhou [134] \\
\hline F. tularensis & Drosophila S2R+ & 2010 & $\begin{array}{l}\text { Akimana } \\
{[135]}\end{array}$ \\
\hline M. tuberculosis & Human Monocyte-like THP-1 & 2010 & Kumar [136] \\
\hline M. tuberculosis & Murine Macrophage-like J774.1 & 2010 & $\begin{array}{l}\text { Jayaswal } \\
{[137]}\end{array}$ \\
\hline B. melitensis & Drosophila SL2 & 2008 & Qin [138] \\
\hline West Nile virus & HeLa & 2008 & $\begin{array}{l}\text { Krishnan } \\
\text { [139] }\end{array}$ \\
\hline C. caviae & Drosophila SL2 & 2007 & Derre [140] \\
\hline L. monocytogenes & Drosophila SL2 & 2005 & $\begin{array}{l}\text { Agaisse } \\
\text { [141] }\end{array}$ \\
\hline M. fortuitum & Drosophila SL2 & 2005 & $\begin{array}{l}\text { Agaisse } \\
\text { [141] }\end{array}$ \\
\hline M. fortuitum & Drosophila SL2 & 2005 & Philips [142] \\
\hline
\end{tabular}


CHAPTER 2:

DEVELOPMENT OF BIOLUMINESCENT BIOREPORTERS FOR IN VITRO AND IN VIVO TRACKING OF YERSINIA PESTIS ${ }^{1}$

\footnotetext{
${ }^{1}$ Sun Y, Connor MG, Pennington JM, Lawrenz MB. Development of bioluminescent bioreporters for in vitro and in vivo tracking of Yersinia pestis. PloS one. 2012;7(10):e47123. PubMed PMID: 23071730.
} 


\section{Overview}

Yersinia pestis causes an acute infection known as the plague. Conventional techniques to enumerate Y. pestis can be labor intensive and do not lend themselves to high throughput assays. In contrast, bioluminescent bioreporters produce light that can be detected using plate readers or optical imaging platforms to monitor bacterial populations as a function of luminescence. Here we describe the development of two Y. pestis chromosomal-based luxCDABE bioreporters, LuXPtolc and LuXPcyszK. These bioreporters use constitutive promoters to drive expression of luxCDABE that allow for sensitive detection of bacteria via bioluminescence in vitro. Importantly, both bioreporters demonstrate a direct correlation between bacterial numbers and bioluminescence, which allows for bioluminescence to be used to compare bacterial numbers. We demonstrate the use of these bioreporters to test antimicrobial inhibitors (LuXPtolC) and monitor intracellular survival (LuXPtolC and LuXP $\left.{ }_{\text {cysZK }}\right)$ in vitro. Furthermore, we show that $Y$. pestis infection of the mouse model can be monitored using whole animal optical imaging in real time. Using optical imaging we observed $Y$. pestis dissemination and differentiated between virulence phenotypes in live animals via bioluminescence. Finally, we demonstrate that whole animal optical imaging can identify unexpected colonization patterns in mutant-infected animals.

\section{$\underline{\text { Introduction }}$}

Bioreporters are engineered microbes that produce a detectable signal that can be used to monitor cell populations or responses to environmental stimuli. The bacterial luxCDABE operon, which produces light through bioluminescence, has been adapted for use as a bioreporter in many species of bacteria [154]. Unlike eukaryotic luciferase systems, the luxCDABE operon produces both the luciferase enzyme and the substrates required for light production, removing the requirement for supplemental exogenous substrates for luminescence [155]. By replacing the native luxCDABE promoter with a promoter from a gene of interest, researchers can monitor changes in gene expression as a function of bioluminescence. lux $C D A B E$ reporters driven by constitutive promoters, in which bacterial density directly correlates to luminescence, provide a system to monitor bacterial growth. Furthermore, because bioluminescence is only produced by viable bacteria, bacterial survival can also be monitored with a luxCDABE reporter [155]. The ease 
of detecting bioluminescent signal from I $u x C D A B E$ without the addition of substrates or inactivation of the bacterium makes this an ideal reporter for real time monitoring of bacteria and high throughput biology technologies.

Yersinia pestis causes the acute infection known as the plague. Human plague can manifest as three different forms. Bubonic plague arises in individuals who have been fed upon by an infected flea. The bacteria are regurgitated into the bite site by the flea and rapidly colonize the proximal lymph nodes. In these tissues, Y. pestis evades the immune system and replicates to high numbers. Without treatment, the bacteria can eventually colonize the bloodstream, leading to the development of septicemic plague. Cases of primary septicemic plague can also arise if $Y$. pestis is directly inoculated into the blood by the flea. From the blood, Y. pestis disseminates to other tissues in the host. Colonization of the lungs results in the development of pneumonia (called secondary pneumonic plague). Pneumonic plague patients can directly transmit $Y$. pestis to naïve individuals via contaminated aerosols, resulting in primary pneumonic plague [156]. Direct aerosol transmission of $Y$. pestis has also raised concerns about the potential use of plague as a biological weapon [14].

Several examples of the use of bioreporters in Yersinia have been reported. Two independent high throughput screens for inhibitors of the Yersinia type III secretion system have used bioluminescent bioreporters. The first screen monitored changes in yop $E$ transcription with a PyopE::/uxAB reporter [157], while the second used a lux operon driven by a constitutive promoter to monitor bacterial growth [158]. Other groups have engineered luxCDABE reporters to be under the transcription control of promoters of virulence genes to monitor expression patterns of these genes [159-161]. In addition to these in vitro assays, a limited number of studies in Yersinia using bioluminescent reporters for optical imaging of whole animals have been reported. Trcek et al. developed an inducible luxCDABE reporter in Y. enterocolitica to monitor oral and IV infection [162]. The authors observed luminescent signal from the abdomen of live animals during oral infection, but due to the nature of the gastrointestinal tract, specific tissue localization required necropsy. However, whole animal imaging revealed unexpected colonization of the cervical lymph nodes that 
has been overlooked using conventional models. In Y. pseudotuberculosis, Thorslund et al. were able to differentiate infection by wild type (WT) or mutant bacteria using the pCD1-Xen4 reporter [163]. More recently, Nham et al. infected animals subcutaneously with WT Y. pestis harboring a plasmid-based luciferase reporter and demonstrated that bioluminescence could be used to localize bacteria to lymph nodes via whole animal imaging. They were also able to use bioluminescence to monitor the development of systemic disease [164].

Whole animal optical imaging has also been used to study pneumonic infection by several Select Agent pathogens. Independently, two groups demonstrated that experimental melioidosis could be visualized in the mouse model $[165,166]$. Furthermore, Warawa et al. were able to visualize both upper and lower respiratory tract colonization, differentiate between colonization patterns of mutant bacteria, and show that luminescence detection from the thoracic cavity strongly correlated to bacterial numbers in the lung. Bina et al. developed a plasmid-based luxCDABE bioreporter in Francisella tularensis[167]. Using this system, they demonstrated that the volume of the bacterial suspension administered to mice could affect whether the bacteria were delivered to the lung [168]. These studies demonstrate the potential for use of bioluminescent-based optical imaging to monitor pneumonic plague.

Several animal models of human plague have been characterized to study $Y$. pestis pathogenesis and develop potential therapeutics [169]. Conventional models to study microbial pathogenesis use separate groups of animals to determine the survival of animals (e.g., LD 50 and/or time to death analysis) or dissemination rate of the pathogen (by enumerating bacteria from specific tissues of subsets of animals sacrificed at various time points). In contrast, optical imaging models allow for temporal and spatial analysis of the infection and survival data to be acquired from the same animal. Potential advantages of optical imagining models are: 1) smaller number of animals required for studies, 2) ability to follow the course of the disease in the same animal over time, and 3) potential to identify unexpected dissemination routes.

Here we describe the development of two chromosomally-based luxCDABE reporters for use in $Y$. pestis. We demonstrate that these reporters can serve as sensitive bioreporters to monitor $Y$. 
pestis growth and survival under different conditions during in vitro growth. We also demonstrate that both bubonic and pneumonic plague infection can be monitored in live animals using these reporters via optical imaging. Finally, we show that the luxCDABE bioreporter can be used to compare and differentiate virulence phenotypes in animals without the need to sacrifice animals.

\section{$\underline{\text { Results }}$}

\section{Construction of a chromosomal luciferase reporter system in Y. pestis}

Our preliminary data demonstrated that in Yersinia a luxCDABE based-reporter was $>200$-fold more sensitive than equivalent fluorescent reporters using dsRED or EGFP (data not shown). Therefore, we developed a bioreporter using the lux operon in Y. pestis. Using a Tn7-based system, we integrated the entire luxCDABE operon driven by the EM7 promoter from pGEN-luxCDABE into the $Y$. pestis chromosome $[170,171]$. Integration of the reporter into the chromosome greatly reduced the amount of bioluminescence produced per bacterium compared to $Y$. pestis with pGENluxCDABE (likely due to a decrease in copy number), resulting in an average limit of detection of $2.84 \times 10^{5} \mathrm{CFU}$ (range $=1.30 \times 10^{4}$ to $6.23 \times 10^{6} \mathrm{CFU}$ ) for the chromosomal reporter (Fig. $2-1 \mathrm{~A}$ and B). To increase the sensitivity, we replaced the EM7 promoter with one of two different promoters. We selected the tolC promoter from Burkholderia pseudomallei, which was used in a similar reporter in B. pseudomallei[165], and the cysZK promoter from Y. pestis, which was identified as a strong constitutive $Y$. pestis promoter [172]. Expression of the luciferase operon from the tolC promoter increased the chromosomal reporter sensitivity by $\sim 100$-fold (average limit of detection $=$ $2.5 \times 10^{3} \mathrm{CFU}$, range $=1.09 \times 10^{3}$ to $\left.5.86 \times 10^{3} \mathrm{CFU}\right)$ and approached the sensitivity of pGENluxCDABE (Fig. 2-1C). The cysZK promoter further increased the sensitivity by an additional 10fold, establishing an average limit of detection of $3.06 \times 10^{2} \mathrm{CFU}$ (range $=1.08 \times 10^{2}$ to $5.76 \times 10^{2} \mathrm{CFU}$ ) (Fig. 2-1D). As reported by Bland et al., we also observed increased expression of PcysZK at $37^{\circ} \mathrm{C}$, but importantly, the LuXPcyszkstrain maintained a direct correlation between bacterial numbers (CFU) and light production (RLU) during continuous growth at both temperatures (Fig. 2-2). LuXPtolC activity did not appear to be influenced by temperature and maintained a strong direct correlation between CFU and RLU at both temperatures (Fig. 2-2). 
To ensure that expression of the lux operon did not affect growth of $Y$. pestis, we determined the growth rate of the $Y$. pestis reporter strains in vitro (Fig. 2-3A). No significant differences were observed between WT Y. pestis (no reporter) or strains carrying the three chromosomal reporters. We further examined whether the Lux reporters impacted fitness of $Y$. pestis in the macrophage model. As seen in broth culture, the Lux reporters did not negatively impact the survival/replication of $Y$. pestis in macrophages, and we observed similar levels of replication by the reporter strains in RAW264.7 macrophages as WT Y. pestis without a reporter (Fig. 2-3B). Together these data demonstrate that integration of the lux operon driven by either PtolC or PcysZK generated a sensitive luciferase reporter that does not appear to impact $Y$. pestis growth and whose light production directly correlates to bacterial number.

\section{Using the Y. pestis Lux reporters as bioreporters}

Due to the requirement for a constant supply of $\mathrm{O}_{2}, \mathrm{FMNH}_{2}$, and aldehydes for the Lux system to produce light, bioluminescence only occurs in actively growing bacteria [155]. This property, in conjunction with the direct correlation between bioluminescence and bacterial numbers for the LuXPtolc and LuXPcysZK reporters, suggests that these reporters can be used to monitor $Y$. pestis survival in real time. To test this hypothesis, we incubated $Y$. pestis LuXPtolc with decreasing concentrations of a chemical disinfectant (MicroChem-Plus), and then monitored bacterial survival as a function of bioluminescence (Fig. 2-4A). At 6 mins post-exposure, samples were harvested, washed and plated to determine if bioluminescence readings correlated with bacterial numbers (Fig. 2-4B). Within 2 mins of exposure to MicroChem-Plus at concentrations $\geq 0.05 \%$, we were unable to detect bioluminescence from the $Y$. pestis cultures. This correlated with viable bacteria, as at these concentrations, viable bacteria were below the level of detection of the LuXPtolc reporter. At levels of MicroChem-Plus $<0.05 \%$ we observed a dose dependent reduction in bioluminescence that directly correlated to the number of bacteria recovered after six mins of incubation.

To further demonstrate that bioluminescence can differentiate bacteria survival, $Y$. pestis LuXPtolc was incubated in 96-well plates with increasing concentrations of carbenicillin or gentamicin. Plates were incubated for $12 \mathrm{hrs}$ at $26^{\circ} \mathrm{C}$, and bioluminescence was detected every hr. 
These readings indicated a dose dependent bacterial growth inhibition, with lower bioluminescence readings observed as antibiotic concentrations increased (Fig. 2-4C and E). To confirm that bioluminescence readings correlated with bacterial numbers, a subset of samples was harvested at 4, 8, and $12 \mathrm{hrs}$ and bacterial CFUs were determined by conventional enumeration (Fig. 2-4D and F). As seen for bioluminescence, we also observed a dose dependent response in bacterial CFU. Together these data demonstrate that bioluminescencecan be used to monitor changes in bacterial survival.

\section{Differentiation between bacterial phenotypes in vitro using Y. pestis Lux bioreporters}

To further demonstrate that the $Y$. pestis Lux bioreporters can be used to monitor bacterial numbers in a biological system, we infected macrophages with WT Y. pestis pCD1(-) or a mutant defective in macrophage survival $(\Delta p h o P)$ carrying our reporter constructs. RAW264.7 macrophages were infected with the reporter strains and extracellular bacteria were killed with gentamicin. At several time points post-infection, bioluminescence was measured using a plate reader. In addition, at $1.5,8$, and $24 \mathrm{hrs}$ post-infection, samples were also harvested to determine bacterial numbers by conventional bacterial enumeration techniques. CFU data demonstrated that all three of the WT Y. pestis reporter strains survived within the macrophages, but the $\triangle p h o P$ mutant strains were attenuated and bacterial numbers differed from WT by approximately two orders of magnitude over the course of the assay (Fig. 2-5A-C). The sensitivity of the bioluminescence signal produced by the LuXPtolc and LuXPcysZK reporter strains allowed for easy differentiation between WT and $\Delta$ phoP phenotypes (Fig. 2-5E-F). In contrast, the lower sensitivity of the LuXPEM7 reporter made it more difficult to differentiate the $\Delta$ phoP phenotype (Fig. 2-5D). While RLU data from the WT LuXPEM7 strain correlated with CFU data, the bioluminescent signal of the $\triangle$ phoP LuXPEM7 strain quickly dropped below the limit of detection of the reporter, resulting in a loss of correlation between bacterial CFU and RLU for this assay. These data demonstrate that the LuXPtolcand LuXPcyszK bioreporters can be used to monitor changes in bacterial populations in biological systems in vitro. 
In vivo imaging of bubonic plague

The high sensitivity of the LuXPcyszK bioreporter that we observed in vitro suggested that it could also be used to monitor plague infection in vivo. Bubonic plague is the most common form of human plague and results from flea transmission. In the laboratory, bubonic plague can be modeled by intradermal or subcutaneous inoculation of mice with $Y$. pestis. After inoculation, the bacteria disseminate to the draining lymph node. Eventually the bacteria enter into the bloodstream to cause a systemic infection. To determine if the LuXPcyszK bioreporter could be used to monitor bubonic infection, specifically lymph node colonization, mice were challenged with the WT CO92 LuXPcyszK strain, and infection was monitored using whole animal optical imaging (Fig. 2-6). Mice were inoculated at the base of the tail with approximately $200-400$ CFU of the bioreporter strain. The sensitivity of the bioreporter strain allowed us to detect bioluminescent signal from the inoculation site as early as $8 \mathrm{hrs}$ post-inoculation. Furthermore, signal increased over time at the inoculation site, indicating that $Y$. pestis survives and replicates at the inoculation site over the course of the infection (Fig. 2-7A).

Previous work has defined the lymphatic drainage basin for the base of the tail to be the subiliac (also referred to as the inguinal) and the axillary lymph nodes (LN) [173, 174]. We began to detect luminescent signal from the subiliac LN starting between 48 and 72 hrs post-inoculation (Fig. 2-6A, white arrows). Approximately 8-15 hrs after first detection in the subiliac LN, signal began to be detected in the axillary LN, indicating bacterial dissemination to these nodes (Fig. 2-6A, red arrows). For both lymph nodes, the bioluminescent signal continued to increase in the tissues over the course of the infection, indicating bacterial proliferation. By $72 \mathrm{hrs}$ post-inoculation, we began to detect bioluminescence from other regions, indicating systemic infection. The animals succumbed to infection by $96 \mathrm{hrs}$ post-inoculation.

To further demonstrate that our bioreporter can be used to monitor bubonic plague dissemination, an additional group of mice was infected in the footpad with the WT CO92 LuXPcyszK strain. Previous work has demonstrated that dyes can disseminate from this site via two different drainage basins in mice $[173,174]$. The first basin drains to the popliteal $L N$, followed by the sciatic 
and renal LNs. Alternatively, drainage to the same basin as from the base of the tail can occur. In these studies we observed $Y$. pestis disseminating only through the former drainage basin from the footpad (Fig. 2-6B). Bioluminescent signal was first detected in the popliteal LN at about $72 \mathrm{hrs}$ post-inoculation. Signal was detected $24 \mathrm{hrs}$ later from regions corresponding to the sciatic and renal LNs. At this time we also were able to detect signal from the spleen. Together these data demonstrate that lymph node colonization and dissemination of $Y$. pestis can be tracked in live animals via optical imaging using the LuXPcyszK bioreporter.

\section{In vivo imaging of pneumonic plague}

Primary pneumonic plague occurs when aerosols containing $Y$. pestis are inhaled by a naïve individual. This form of disease can also be modeled in the mouse using the intranasal route of infection [175]. To determine if the LuXPcyszk bioreporter can be used to monitor pneumonic infection, we challenged mice intranasally with the WT CO92 LuXPcyszK strain and followed the progression of pneumonic plague by optical imaging. Bioluminescent signal could be detected from the thoracic cavity of all mice as early as $24 \mathrm{hrs}$ post-inoculation and increased throughout the course of infection (Fig. 2-8A and B). To demonstrate that the bioluminescence signal directly correlated with bacterial numbers, lungs were harvested from a subset of animals after the 24,48 , and 72 hrs imaging sessions. The tissues were imaged and bacterial numbers in the lungs were determined. Bioluminescent signal from imaging of the thoracic cavity directly correlated to lung CFU (Fig. 2-8C; $R^{2}=0.8323$ ). The significance of the correlation increased further when comparing signal directly from harvested lungs to CFU (Fig. 2-8D; $R^{2}=0.9684$ ). Animals infected with the LuXPcysZK Strain succumbed to infection between 60 and $80 \mathrm{hrs}$ post-infection, a similar time to death as seen for $Y$. pestis without a reporter $[175,176]$.

\section{Differences in phenotypes can be detected in vivo using the LuX ${ }_{\text {PcyszK }}$ bioreporter}

To demonstrate that whole animal imaging using the LuXPcyszK bioreporter can differentiate between virulence phenotypes, we transferred the reporter into a $Y$. pestis $\Delta$ pla mutant. Pla is required for the development of bubonic plague, and a pla mutant is unable to disseminate from the inoculation site to the draining LN [177-179]. In the bubonic model, we observed bioluminescent 
signal from the inoculation site of $Y$. pestis $\Delta$ pla LuXPcyszK infected animals as early as 8 hrs postinfection (Fig. 2-7A). Signal increased at the inoculation site at a rate comparable to WT infected animals until 36 hrs post-infection. After 36 hrs, signal from WT infected animals continued to increase, but the signal from $\Delta$ pla infected animals plateaued, remaining about 1-2 logs lower than WT signal for the remainder of the experiment. No signal was observed from the draining LN from $\Delta$ pla infected animals (Fig. 2-7B), supporting previous data that the mutant is unable to disseminate to the LN after intradermal infection [179]. However, one $\Delta$ pla infected animal $(n=9)$ appeared to develop primary septicemic plague, as no signal was detected from the lymph nodes prior to systemic signal (data not shown).

In the model for pneumonic plague, the $\Delta$ pla mutant colonizes the lungs but is unable to proliferate in these tissues [176]. As expected, we observed low levels of bioluminescence from the thoracic cavity of mice infected intranasally with $Y$. pestis $\Delta$ pla LuX $\mathrm{P}_{c y s Z K}$, correlating with low levels of bacteria in these tissues (Fig. 2-8). Importantly, compared to WT infected mice, luminescence from the $\Delta$ pla infected animals was significantly lower at all time points, except at the $72 \mathrm{hr}$ time point when there were not enough WT animals to calculate significance (Fig. 2-8B). While the $\Delta$ pla mutant does not proliferate within the lungs during pneumonic infection, the $L_{50}$ of the mutant is similar to WT Y. pestis, likely due to thedevelopment of septicemic plague [176]. The sensitivity of the LuXPcyszk bioreporter allowed us to observe the development of septicemic plague in $\Delta$ pla infected animals (Fig. 2-9A). Furthermore, as we monitored the $\Delta$ pla infected animals, we also observed that a subset of animals developed bioluminescent signal near the ears which we did not observe in WT infected animals (Fig. 2-9). Together these data demonstrate that whole animal imaging with the LuX $\mathrm{P} c y s z K_{\mathrm{K}}$ bioreporter can differentiate between bacterial phenotypes during both bubonic and pneumonic plague infection.

\section{Discussion}

The bacterial luxCDABE operon produces a bioluminescent signal that can be used as a bioreporter to monitor bacterial numbers in real time. We developed two luxCDABE reporters for use in $Y$. pestis to monitor bacterial survival. We demonstrated that these reporters can be used to 
monitor bacterial numbers in the presence of antimicrobial compounds, during intracellular infection, and in animal models for plague infection. Unlike plasmid-based systems previously used in Yersinia spp. [160, 162-164], these reporters are integrated into the chromosome. A chromosomal-based system has several characteristics that may be advantageous for future applications. First, integration of the reporter into the chromosome does not require antibiotic selection for maintenance and will likely be more stable than a plasmid-based system. Second, while plasmid reporters may be maintained without antibiotics for a period of time, especially with integrated toxin-anti-toxin maintenance mechanisms [164], [180], the plasmid still confers resistance for the selectable marker carried by the plasmid. Consequently, that marker is not available for further use (for example, to maintain other plasmids). The chromosomal reporters described here were engineered using a system that allows for the antibiotic marker to be removed after integration [171]. Therefore, the marker (in this case Kan) can be reused in downstream applications.

One advantage of a plasmid-based reporter system is that plasmids are often maintained at increased copy numbers compared to the chromosome, which can increase the sensitivity of the reporter. In fact, we observed a dramatic decrease in sensitivity when we moved the luxCDABE operon from a plasmid to the chromosome. To overcome this problem we removed the promoter from the original construct and replaced it with a promoter we hypothesized would increase the expression of the lux operon. We chose two different promoters to test. The first promoter was from B. pseudomallei (PtolC) and had been used to successfully develop a similar chromosomal reporter for this bacterium [165]. This promoter increased the sensitivity to the levels of the original plasmidbased reporter. The second promoter was originally identified by Bland et al. as being a strong constitutive promoter in Y. pestis (PcysZK) [172]. This promoter further increased the sensitivity to a level approximately 10 -fold higher than the LuXPtolc or pGEN-luxCDABE. Importantly, we saw no deleterious impact of increased luxCDABE expression from our reporters on $Y$. pestis fitness during growth in vitro, in cell culture, or in the animal models. Therefore, we successfully engineered a chromosomal luciferase reporter that is 10 -fold more sensitive than a widely used plasmid-based reporter, without attenuating growth of $Y$. pestis. 
For both the LuXPtolc and LuXPcysZK reporters we observed a direct correlation between bioluminescence and $Y$. pestis numbers. This characteristic is important and demonstrates that bioluminescence readings from these reporters can be used to quantify bacterial numbers. Furthermore, the sensitivity of the reporter and easy detection methods allow these bioreporters to be used in large scale formats. For example, we demonstrated that we could use the Luxptolc bioreporter to monitor bacterial growth in a 96-well format in the presence of antimicrobial compounds. Using this format we were able to easily determine the MIC for both carbenicillin and gentamicin. Furthermore, because we could monitor the bacteria in real time, we were also able to observe differences in growth patterns of $Y$. pestis in these two antibiotics. For example, $Y$. pestis incubated in inhibitory concentrations of carbenicillin $(12.5$ and $25 \mu \mathrm{g} / \mathrm{ml})$ did not begin to decrease in bioluminescence until after 8 hrs into the assay, indicating that while bacterial growth might be inhibited, the bacteria were not killed by the antibiotic until after that time (Fig. 2-4C). In contrast, bioluminescence signal from bacteria incubated with inhibitory concentrations of gentamicin $(2,4$, and $8 \mu \mathrm{g} / \mathrm{ml}$ ) steadily decreased over the course of the assay, suggesting that bacterial death occurred much earlier (Fig. 2-4E). These hypotheses are supported by the CFU data that demonstrated that bacterial numbers did not begin to decrease in the carbenicillin samples until between 8 and 12 hrs, compared to between 4 and 8 hrs in gentamicin samples (Fig. 2-4D and F). These phenotypes can be explained by the mechanisms of action of the two antibiotics. Gentamicin blocks protein synthesis and quickly inhibits bacterial growth, whereas carbenicillin targets the bacterial peptidoglycan, which over time weakens the cell wall, leading to osmotic lysis, but allows for a short period of proliferation. The sensitivity and correlation between bioluminescence and bacterial numbers indicate that the bioreporters can be used to monitor $Y$. pestis survival in high throughput screens for new anti- $Y$. pestis compounds.

While we saw a consistent correlation between bioluminescence and bacterial CFU in all of the assays we reported, macrophages infected with $\triangle p h O P$ LuXPcyszK demonstrated a decrease in bioluminescence between 8 and 24 hrs without a significant difference in CFU between these two time points. The same phenotype was not observed in the WT LuXPcyszK strain or in the LuXPtolC strains, all of which maintained correlation between RLU and CFU (Fig. 2-5). These observations 
demonstrate that depending on the specific experimental assay, one bioreporter may more accurately represent bacterial numbers than the other. Furthermore, while the $Y$. pestis LuX $\mathrm{P}_{\text {cys } Z K}$ bioreporter was more sensitive than the Luxptolc bioreporter in our initial studies (Fig. 2-1), sensitivities of the bioreporters may change under different experimental conditions. For example, we observed that LuXPcyszK is more active at $37^{\circ} \mathrm{C}$ than $26^{\circ} \mathrm{C}$. Therefore, optimization and validation of the bioreporters must be performed for each new assay as it is being developed.

Nham et al. recently reported the use of a plasmid-based bioluminescent bioreporter to follow the progression of bubonic plague in mice [164]. Using this bioreporter they demonstrated that spread of $Y$. pestis to the draining lymph nodes could be visualized in live animals via optical imaging. Furthermore, the authors were able to identify spread to the liver and spleen during disseminated (septicemic) plague. Similarly, we demonstrate here that the LuXPcyszkbioreporter could be used in optical imaging of bubonic infection. The sensitivity of the LuXPcyszkbioreporter allowed detection of bacteria at the inoculation site as early as $8 \mathrm{hrs}$ post-infection, and we observed distinct dissemination patterns of $Y$. pestis LuXPcyszk from two different inoculation sites that followed the predicted lymphatic drainage basins. As the infection progressed, we were able to identify the transition to systemic infection when bioluminescence was detected from the spleen. Eventually bioluminescence was detected from more peripheral sites, such as the feet and tail, demonstrating that bacterial concentrations reached levels in the blood that could be detected by optical imaging prior to the animals succumbing to infection.

Our data also demonstrate that WT bacteria are not cleared from the inoculation site over the course of the infection, and continuous increase of bioluminescence at the site indicates that the bacteria proliferate. It is still unclear whether secondary septicemic plague initiates from bacteria disseminating from the lymph nodes or the inoculation site, but our data suggest that viable bacteria remain at the inoculation site as a possible reservoir for septicemic spread. Interestingly, while the $\Delta$ pla mutant did not appear to proliferate to WT levels at the inoculation site, we continued to detect bioluminescent signal from this site for as long as 14 days post-inoculation (unpublished data). These data indicate that the mutant can survive at the inoculation site for an extended period of 
time, but survival at this site was not sufficient to lead to septicemic plague. However, one of the nine animals infected with the $\Delta$ pla mutant developed septicemic plague during our studies. The lack of detectable signal from the draining lymph nodes suggests that the bacteria disseminated into the bloodstream without first colonizing the lymph nodes. A similar rate of septicemic infection by the $\Delta$ pla mutant was previously reported by Sebbane et al. [179]. While these data may suggest septicemic plague arises from the inoculation site, we agree with Sebbane et al. that it is more likely that sepsis resulted from direct inoculation of the bacteria into the bloodstream during the infection and not from escape from the inoculation site. Additional studies are needed to further understand the dissemination of $Y$. pestis into the bloodstream.

In addition to bubonic infection, we also demonstrate that the LuX $\mathrm{P} c y s z K_{\mathrm{Z}}$ bioreporter is sensitive enough to monitor infection of deeper tissues colonized during pneumonic plague. Importantly, through enumeration of bacterial CFU in the lungs, we demonstrated that bioluminescence from the thoracic cavity directly correlates to bacterial numbers in the lungs. This correlation supports the use of bioluminescence to estimate bacterial burden in the lungs. Furthermore, we were easily able to differentiate between WT and $\Delta$ pla infected animals, suggesting that this bioreporter can be used to differentiate between mutant phenotypes in the animal. The ability to monitor the entire progression of plague in an individual animal via optical imaging allows for dissemination kinetics and survival data to be obtained from the same group of animals, resulting in smaller number of animals per experiment. Furthermore, optical imaging of plague with the LuX $\mathrm{P}_{c y s z K}$ bioreporter may benefit therapeutic research, as it will allow researchers to observe the resolution of an established infection after treatment is initiated.

Optical imaging with the LuXPcyszkbioreporter will also allow researchers to identify unexpected dissemination patterns that might be missed in conventional models. For example, in a subset of animals intranasally infected with the $\Delta$ pla mutant, we observed bioluminescence from a region near the ears, which we did not observe in WT infected animals. The precise tissues infected in these animals have yet to be identified, but colonization of tissues in this region would not have been detected without the whole animal imaging data. These data raise the possibility that systemic 
infection by the $\Delta$ pla mutant may arise from colonization of the upper respiratory tract as opposed to dissemination directly from the lungs. However, additional experiments to determine the frequency of this phenotype, correlation to systemic infection, and identity of infected tissues are required to test this hypothesis. 


\section{Material and Methods}

\section{Bacterial strains, plasmids, and growth conditions}

The bacterial strains and plasmids used in this study are listed in Table 2-1. E. coli was grown in Luria-Bertani (LB) broth at $37^{\circ} \mathrm{C}$. Y. pestis was grown in Brain Heart Infusion (BHI) broth at 26 or $37^{\circ} \mathrm{C}$ (with $2.5 \mathrm{mM} \mathrm{CaCl}_{2}$ ). When appropriate, antibiotics were used at the following concentrations: kanamycin, $50 \mu \mathrm{g} \mathrm{ml}^{-1}$ (E. coli), $25 \mu \mathrm{g} \mathrm{ml}^{-1}$ (Yersinia); carbenicillin, $50 \mu \mathrm{g} \mathrm{ml}^{-1}$.

The $Y$. pestis phoP mutant was generated using lambda red recombinase as previously described [181]. Briefly, regions flanking the phoP gene were amplified by PCR with primers DNA418 (5'-GAT TTC TAC ACC GTC GTG GG-3') and DNA419 (5'-GAA GCA GCT CCA GCC TAC AC CAT ACA CCA ATC CTT GAT AAA ACG TTA AC-3')for the 5' fragment and primers DNA420 (5'-GGT CGA CGG ATC CCC GGA ATAG ACA CTA TGC TCA GAA AAA ATA ATA AAC CC-3') and DNA421 (5'-GGT GAG TTG AGG TAA ACG AGA G-3') for the 3' region. The resulting products were gel purified and combined with a kan cassette flanked by FRT sites via overlapping extension PCR using primers DNA418 and DNA421. The resulting fragment was transformed into YPA035 expressing lambda red recombinase, followed by excision of the kan cassette, to generate YPA047.

The chromosomal luxCDABE reporters (Lux) were generated by first amplifying the lux operon, including the EM7 promoter, from pGEN-luxCDABE by PCR using primers DNA398 (5'-G GAG CTCCTC TGT CAT TTT CTG AAA CTC TTC ATG CTG-3') and DNA399 (5'-G GAG CTCCCG CAT CAA CTA TCA AAC GCT TCG-3') (engineered Sacl restriction sites are underlined) [170]. The PCR product and pUC18r6k-mini-Tn7(kanEW) (a derivative of pUC18r6k-mini-Tn7 [171]in which the original kan cassette was replaced with the kan cassette from pKD13) were digested with Sacl and ligated together to generate pLOU027. The EM7 promoter was subsequently removed from pLOU027 by digesting the plasmid with $\mathrm{Kpnl}$, which excised the promoter. The tolC promoter was amplified by PCR using primers DNA408 (5'-G GGT ACCGCC ACT CAT CGC AGT GTG-3') and DNA409 (5'-G GGT ACCAGG ATC GTC AAA AAC CGA TAT AAG ACG-3') and the cysZK promoter using primers DNA406 (5'-G GGT ACC ACT CTC GCC AAT ATT ATT GCG G-3') and 
DNA407 (5'-G GGT ACCCGC CAA AAT ACG TCC GTT G-3') (engineered Kpnl restriction sites are underlined). PCR products were digested with Kpnl and ligated into Kpnl-digested pLOU027. Proper orientation of the promoters was confirmed by DNA sequencing. Reporters were integrated into the $Y$. pestis chromosome through site specific transposition as described previously to generate the LuXPEM7, LuXPtolC, and LuXPcyszK bioreporter strains [171]. The antibiotic resistance cassette was excised from MBLYP-043 and MBLYP-045 as described previously [181].

To compare the sensitivity of the reporters, reporter strains YPA022, YPA038, YPA039, and YPA040 were inoculated in $\mathrm{BHI}$ broth in triplicate and grown for $15 \mathrm{hrs}$ at $26^{\circ} \mathrm{C}$. Serial 10 -fold dilutions of the cultures were made in sterile 1 X PBS, and the bacterial concentration of the dilutions was determined by enumerating on $\mathrm{BHI}$ agar. $100 \mu \mathrm{l}$ aliquots were also transferred to a 96 -well white plate and bioluminescence for each dilution was determined using a Synergy HT plate reader (BioTek, Winooski, VT) (1 sec read, sensitivity of 135). Linear regression analysis of the log transformed data was used to calculate the trend line, $\mathrm{R}^{2}$ values, and limit of detection.

To determine growth profiles and correlation between CFU and bioluminescence, YPA035, YPA038, YPA039, and YPA040 were grown for $15 \mathrm{hrs}$ in $\mathrm{BHI}$ at $26^{\circ} \mathrm{C}$. Bacteria were diluted into fresh medium to a concentration of 0.03 to $0.05 \mathrm{OD}_{600} / \mathrm{ml}$ and grown for $12 \mathrm{hrs}$ at either 26 or $37^{\circ} \mathrm{C}$. Samples were harvested at various time points during growth to determine $\mathrm{OD}_{600}$, bioluminescence using a Synergy HT plate reader (1 sec read, sensitivity of 135), and bacterial numbers by serial dilution and enumeration on $\mathrm{BH}$ agar. Linear regression analysis of the log transformed data was used to calculate the trend line and $R^{2}$ values. To compare expression between 26 and $37^{\circ} \mathrm{C}, \mathrm{RLU}$ per CFU was determined for each sample over the entire growth curve. Statistical significance was determined using the Mann-Whitney $t$ test with a two-tailed nonparametric analysis.

\section{Survival of $Y$. pestis in the presence of antimicrobial compounds}

To monitor survival of $Y$. pestis in antimicrobial compounds, YPA039 was grown for $15 \mathrm{hrs}$ at $26^{\circ} \mathrm{C}$. The $\mathrm{OD}_{600}$ of the culture was determined and bacteria were diluted to $1 \mathrm{OD}_{600} / \mathrm{ml}$. Bacteria were further diluted 100 -fold in $\mathrm{BHI}$ to a final concentration of $\sim 10^{6} \mathrm{CFU} / \mathrm{ml} .100 \mu \mathrm{l}$ of bacteria were added to wells of a white 96-well plate. Bioluminescence for each well was determined with a 
Synergy HT plate reader (1 sec read, sensitivity of 135) to establish a baseline and then $100 \mu \mathrm{l}$ of indicated dilutions of MicroChem-Plus (National Chemical Laboratories, Philadelphia, PA) or antibiotics (diluted in $\mathrm{BHI}$ ) were added to each well. For MicroChem-Plus, the first reading was taken 2.5 mins after addition and every 1.3 mins thereafter until 14 mins. At 6 mins, a subset of samples was harvested, washed once with 1X PBS, and 10-fold serial dilutions of bacteria were spot plated on $\mathrm{BHI}$ agar. For antibiotics, the first reading was taken 10 mins after addition of antibiotics and every $\mathrm{hr}$ thereafter for $15 \mathrm{hrs}$. Plates were incubated at $26^{\circ} \mathrm{C}$ in the plate reader between reads. Samples were blanked against $\mathrm{BHI}$ only wells. At 4, 8, and $12 \mathrm{hrs}, 100 \mu \mathrm{l}$ of bacteria were harvested from each concentration and 10-fold serial dilutions were spot plated on $\mathrm{BHI}$ agar to determine CFU.

\section{Intracellular survival assays}

RAW264.7 macrophages (ATCC, Manassas, VA) were seeded into white 96-well tissue culture plates and infected with $10^{6} \mathrm{CFU}(\mathrm{MOI}=10)$ of the $Y$. pestis reporter strains YPA035, YPA038, YPA039, YPA040, YPA073, YPA048, or YPA049, as described previously [182]. Extracellular bacteria were killed by incubation with gentamicin $(16 \mu \mathrm{g} / \mathrm{ml})$ for $1 \mathrm{hr}$, followed by three washes

with 1 X PBS. Medium was replaced with DMEM+10\% FBS containing $2 \mu \mathrm{g} / \mathrm{ml}$ gentamicin and plates were incubated at $37^{\circ} \mathrm{C}$ with $5 \% \mathrm{CO}_{2}$ for $24 \mathrm{hrs}$. Bioluminescence was determined at various time points using a Synergy HT plate reader (1 sec reading, sensitivity of 135). For CFU determinations, cells were lysed with $1 \%$ Triton 100 and bacteria were enumerated by serial dilution and plating on $\mathrm{BHI}$ agar.

$\underline{\text { In vivo imaging }}$

All animal studies were approved by the University of Louisville Institutional Animal Care and Use Committee (protocol 10-117). Five-to 7-week-old female B6(Cg)-Tyrc-2J/J (albino C57BI/6) mice (The Jackson Laboratory,Bar Harbor, ME) were maintained in the ABSL-3 vivarium with sterilized food and water adlibitum at the University of Louisville's Center for Predictive Medicine Regional Biocontainment Laboratory and imaging was performed in conjunction with the Center for Predictive Medicine BIO-Imaging Core. Hair was removed with clippers on the dorsal and ventral 
sides of the mice two days prior to infection. Mice were anesthetized using a ketamine-xylene mixture for infections and isoflurane for imaging. Mice were infected with MBLYP-043 (WT) or MBLYP-045 ( $\Delta$ pla). For bubonic studies, mice were infected via injection of 200-400 CFU at the base of the tail or in the hind foot. For pneumonic infections, mice were infected via intranasal infection of $10^{4}-10^{5} \mathrm{CFU}$. Beginning after infection, mice were monitored for disease symptoms twice daily and moribund mice were euthanized. For imaging, mice were anesthetized and images were taken using the IVIS Spectrum imaging system (Caliper Life Sciences, Hopkinton, MA). Average radiance (photons $/ \mathrm{sec} / \mathrm{cm}^{2}$ ) was calculated for regions of interest of infected animals and similar regions were analyzed from uninfected animals or tissues to determine background luminescence (used as the limit of detection). Statistical significance was determined using the Mann-Whitney $t$ test with a two-tailed nonparametric analysis. 


\section{Figures and Figure Legends}

A

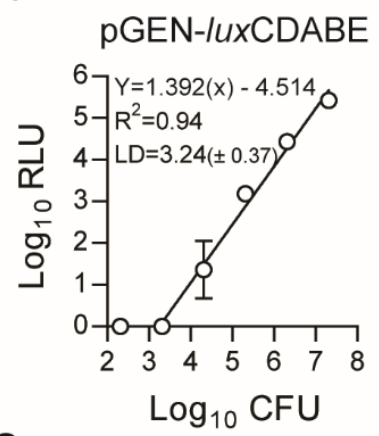

C

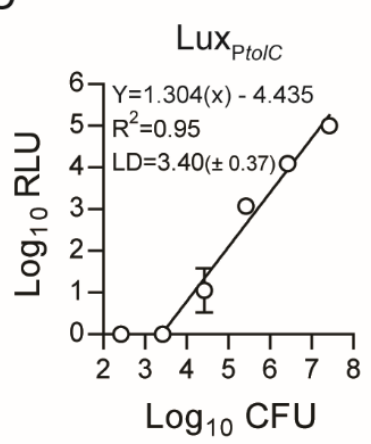

B

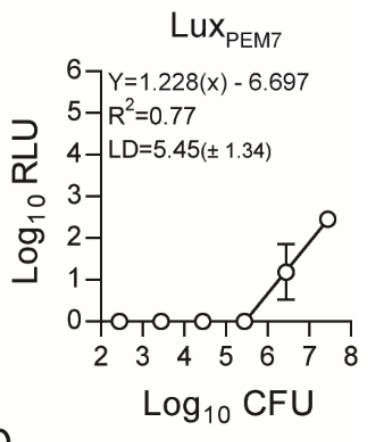

D

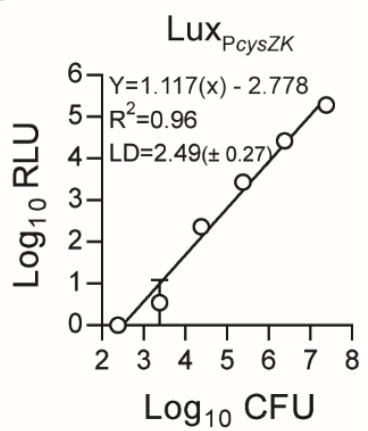

Figure 2-1Sensitivities of chromosomal Lux reporters.

The luxCDABE operon driven by different promoters was integrated into the $Y$. pestis chromosome using Tn7 transposition. Sensitivities of the Lux reporters were determined by making serial dilutions of the $Y$. pestis Lux strains (grown for $15 \mathrm{hrs)} \mathrm{and} \mathrm{determining} \mathrm{the} \mathrm{number} \mathrm{of} \mathrm{bacteria}$ (CFU) and bioluminescence (RLU) in each dilution $(n=3)$. Linear regression analysis of the Log transformed data was used to calculate the trend line, $\mathrm{R}^{2}$ values, and the limit of detection [LD=Log ${ }_{10} \mathrm{CFU}$ ( \pm standard deviation)]. (A) pGEN-luxCDABE, (B) LuXPEM7, (C) LuXPtolc, (D) LuXPcysZK. 
A

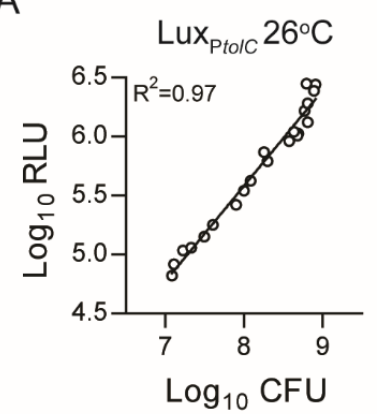

C

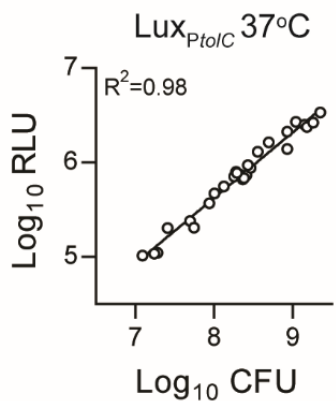

E

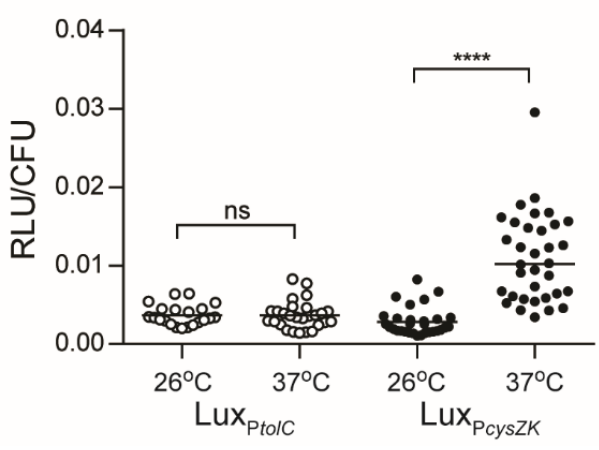

B

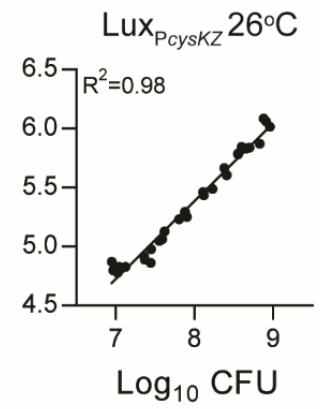

D

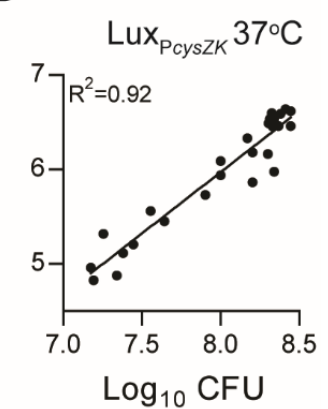

Figure 2-2Correlation between bioluminescence and bacterial number.

Y. pestis LuXPtolc and LuXPcyszkwere diluted in $\mathrm{BHI}$ broth $(\mathrm{n}=3)$ and grown at $26^{\circ} \mathrm{C}(\mathrm{A}$ and $\mathrm{B})$ or $37^{\circ} \mathrm{C}$ (C and D) for $12 \mathrm{hrs.} \mathrm{Samples} \mathrm{were} \mathrm{harvested} \mathrm{at} \mathrm{multiple} \mathrm{time} \mathrm{points} \mathrm{during} \mathrm{growth} \mathrm{to} \mathrm{determine}$ bioluminescence (RLU) and bacterial numbers (CFU). Linear regression analysis of the Log transformed data was used to calculate the trend line and $R^{2}$ values. (E) To determine if

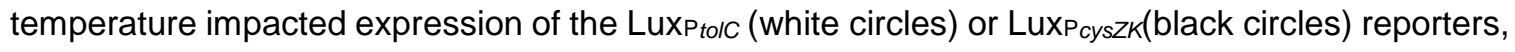
we calculated the RLU/CFU for each sample in A-D and compared the ratios. Black bars represent median values and statistical significance was determined using the Mann-Whitney $t$ test with a two-tailed nonparametric analysis $\left({ }^{* * * *}=p<0.0001, n s=\right.$ not significantly different) 

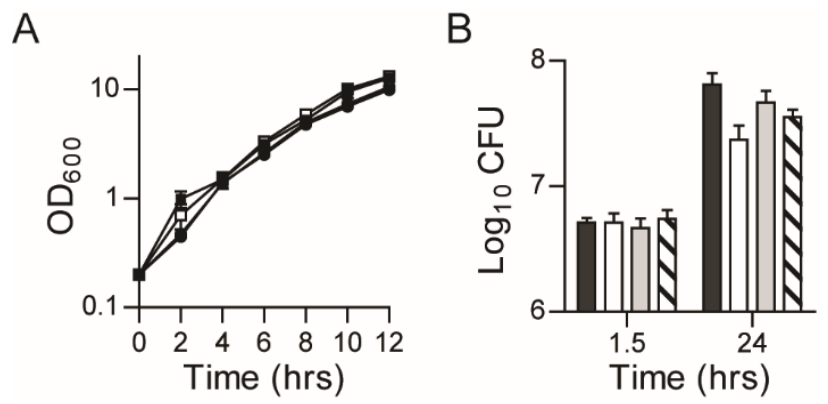

Figure 2-3Lux reporters do not impact fitness of $Y$. pestis.

To determine if carriage of the Lux reporters impacted $Y$. pestis fitness, (A) growth of the $Y$. pestis Lux bioreporter strains $(n=3)$, and (B) survival in macrophages $(n=3)$ were compared to WT $Y$. pestis without a Lux reporter. WT (no reporter) $=\bullet$ or black bar; LuXPEM7 $=\circ$ or white bar; LuXPtolc $=\square$ or gray bar; LuXPcysZK $=\mathbf{\square}$ or hatched bar. 
A
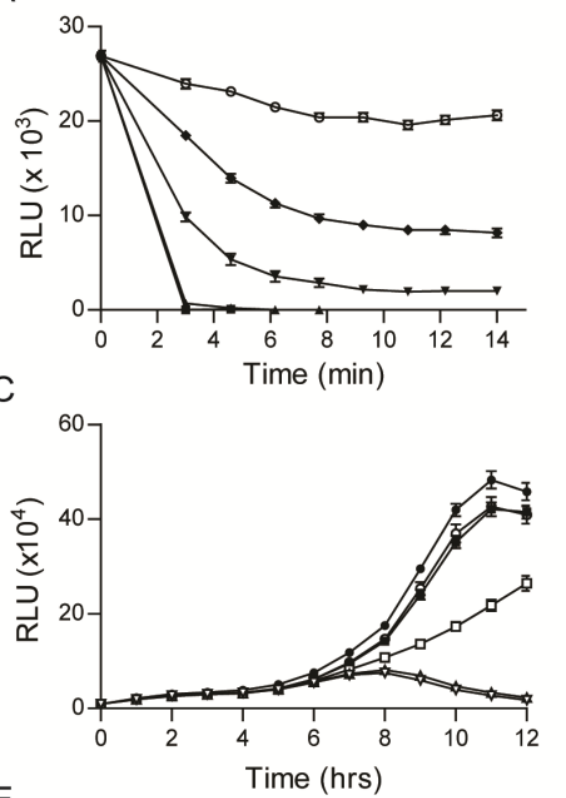

$\mathrm{E}$

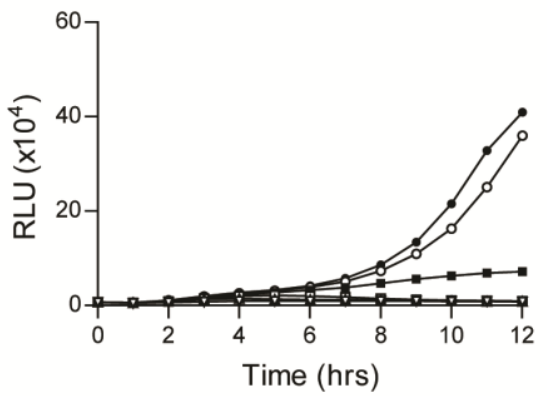

B

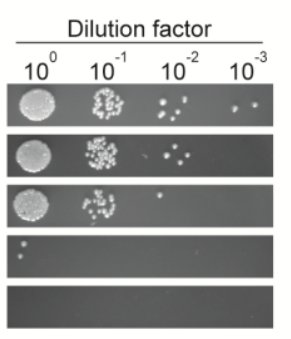

D

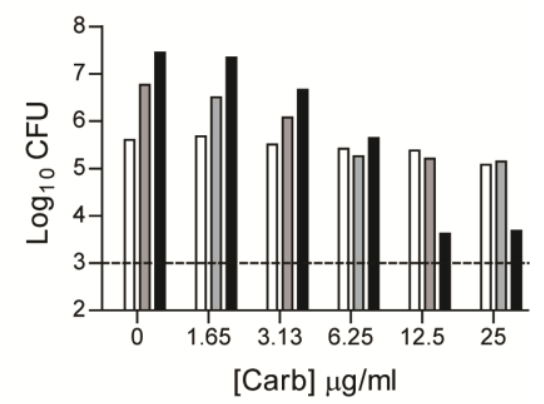

$\mathrm{F}$

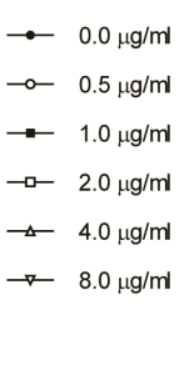

$\rightarrow 0.00 \mu \mathrm{g} / \mathrm{m}$

$\multimap 1.65 \mu \mathrm{g} / \mathrm{ml}$

$\because-3.13 \mu \mathrm{g} / \mathrm{ml}$

$\rightarrow-6.25 \mu \mathrm{g} / \mathrm{m}$

$\rightarrow-12.5 \mu \mathrm{g} / \mathrm{ml}$

$\rightarrow 25.0 \mu \mathrm{g} / \mathrm{ml}$

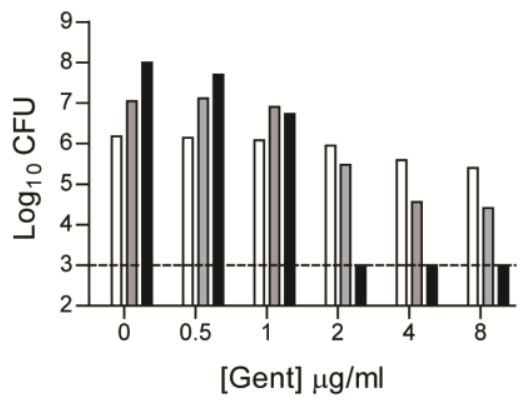

Figure 2-4Use of LuxPtolC to monitor survival of $Y$. pestis in the presence of antimicrobial compounds.

Y. pestis LuXPtolcwas incubated with increasing concentrations of antimicrobials $(n=9)$ in a 96-well format and bacterial survival was monitored by measuring bioluminescence. (A) Bioluminescence readings (RLU) from Y. pestis LuXPtolcincubated with MicroChem-Plus for 14 mins. (B) At 6 mins during incubation with MicroChem-Plus, bacteria were harvested from a subset of wells, washed, serially diluted, and spot plated on agar to determine bacterial CFU. (C) Bioluminescence readings (RLU) from Y. pestis LuXPtolcincubated with carbenicillin for 12 hrs. (D) At 4 (white), 8 (gray), and 12 (black) hrs during incubation with carbenicillin bacteria were harvested from a subset of wells to determine bacterial CFU. (E) Bioluminescence readings (RLU) from Y. pestis LuXPtoldincubated with gentamicin for 12 hrs. (F) At 4 (white), 8 (gray), and 12 (black) hrs during incubation with gentamicin, bacteria were harvested from a subset of wells to determine bacterial CFU. For D and $\mathrm{F}$, the dotted line represents the limit of detection. 

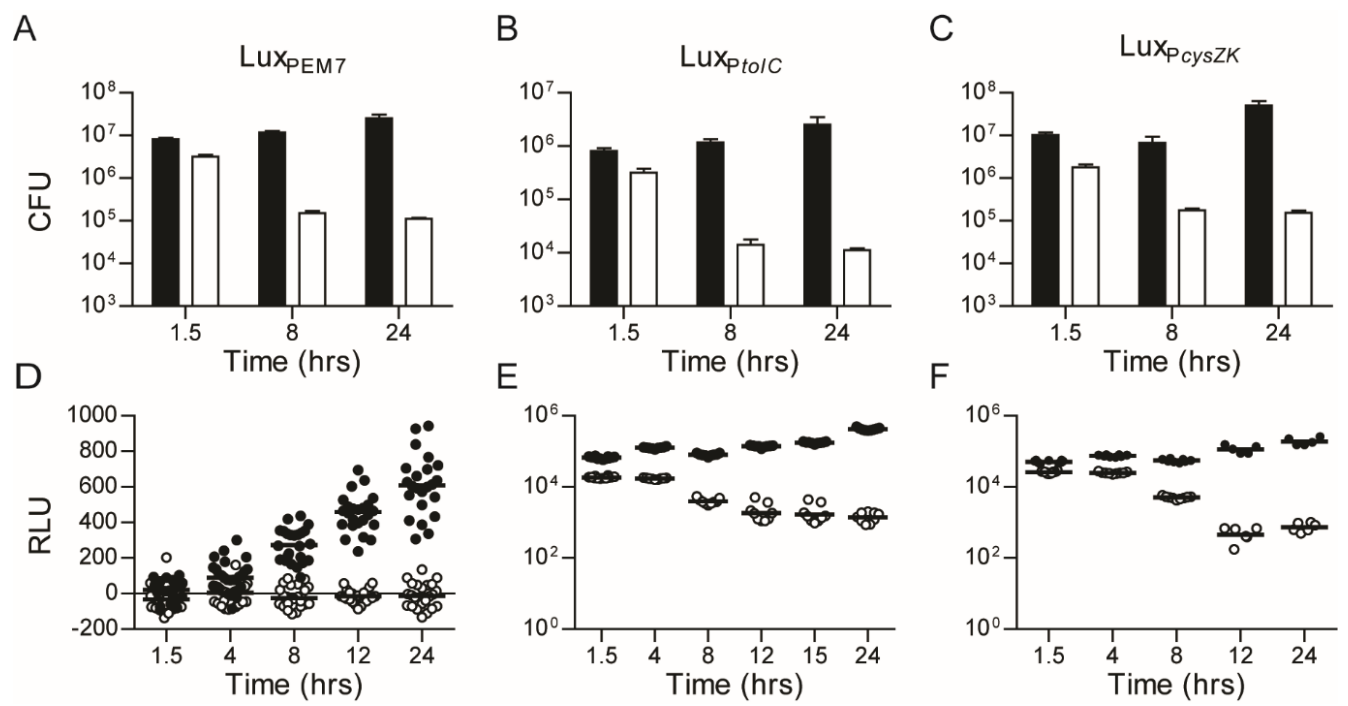

Figure 2-5. Survival of $Y$. pestis Lux reporters in macrophages.

RAW264.7 macrophages were infected with Y. pestis Lux reporter strains, extracellular bacteria killed by gentamicin, and bacterial survival monitored by CFU determination (A-C) or bioluminescence (D-F). Data from WT Y. pestis is represented by black symbols and from an attenuated $\triangle$ phoP mutant by white symbols. (A and D) are strains with the LuXPEM7 reporter $(\mathrm{n}=3$ for CFU, $n=24$ for $\mathrm{RLU})$, (B and $E$ ) are strains with the LuXPtoldreporter ( $n=3$ for $C F U, n=12$ for $R L U)$, and ( $C$ and $F$ ) are strains with the LuXPcyszkreporter ( $n=3$ for CFU, $n=12$ for RLU). 
A

Base of Tail
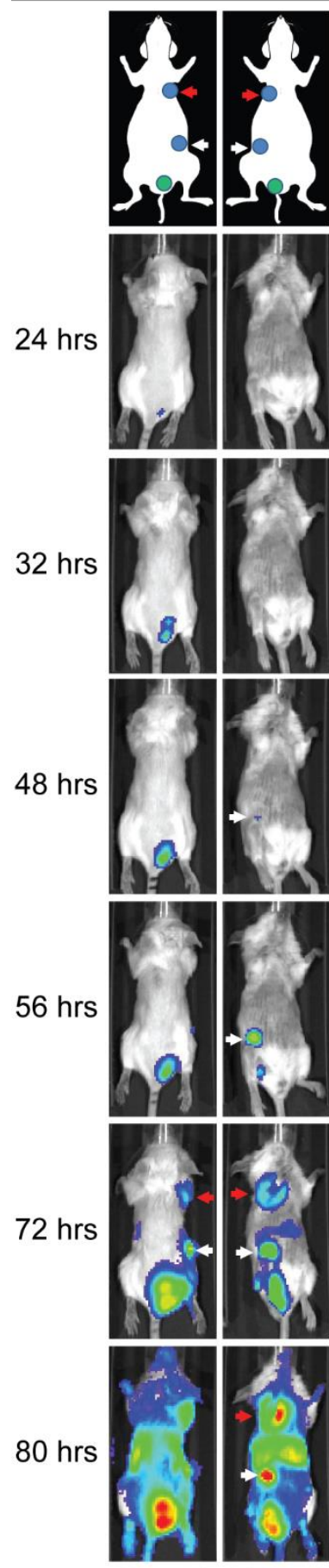

Dorsal Ventral
B

\section{Footpad}

$72 \mathrm{hrs}$

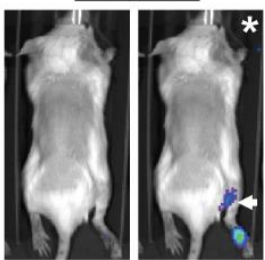

$80 \mathrm{hrs}$

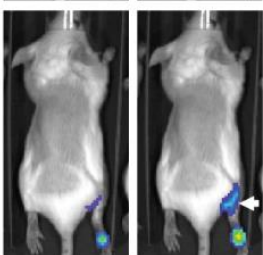

$96 \mathrm{hrs}$

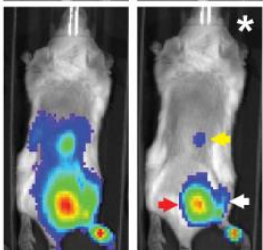

$104 \mathrm{hrs}$
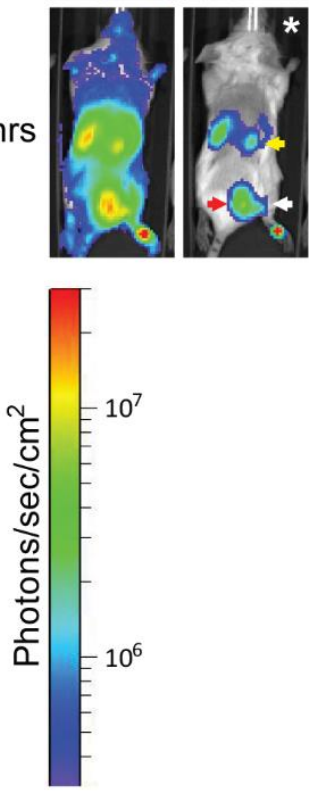

Figure 2-6. Dissemination of $Y$. pestis during bubonic infection.

Mice were infected with $\sim 200 \mathrm{CFU}$ of Y. pestis LuXPcyszK subcutaneously at the base of the tail (A) or in the footpad (B) and imaged using an IVIS Spectrum. The lymph node drainage basin for each 
inoculation site is diagrammed above the images [173, 174]. Location of the inoculation site is shown as a green circle, lymph nodes as blue circles, and the spleen as a red oval. For (A), the white arrow denotes the subiliac LN and the red arrow the axillary LN. For (B), the white arrow denotes the popliteal LN, the red arrow the sciatic LN, and the yellow arrow the renal LN. All images were adjusted to the radiance scale shown, except for the images in (B) marked with * in upper right corners. For these each image was adjusted to a different radiance to allow for visualization of specific tissues. 


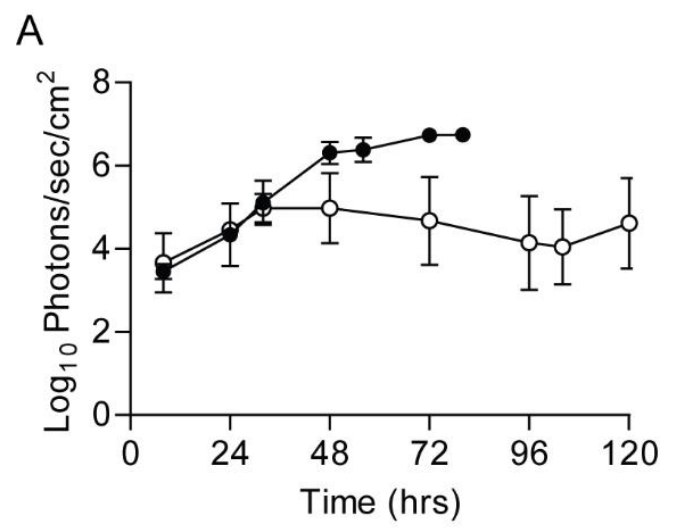

B

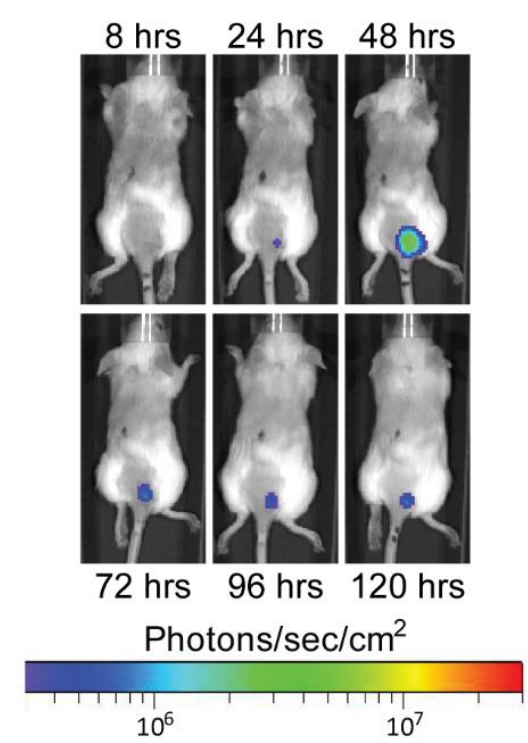

Figure 2-7. Continued bioluminescence from inoculation site.

Mice were infected with $\sim 200$ CFU of WT $(n=5)$ or $\Delta p l a(n=5)$ Y. pestis LuXPcysZK subcutaneously at the base of the tail and imaged using an IVIS Spectrum. (A) The average bioluminescence detected from the inoculation site was determined over the course of the infection. Black and white symbols represent animals infected with WT or $\Delta$ plaY. pestis, respectively. (B) Sequential images from a representative animal infected with $\Delta$ plaY. pestis LuXPcyszK. 

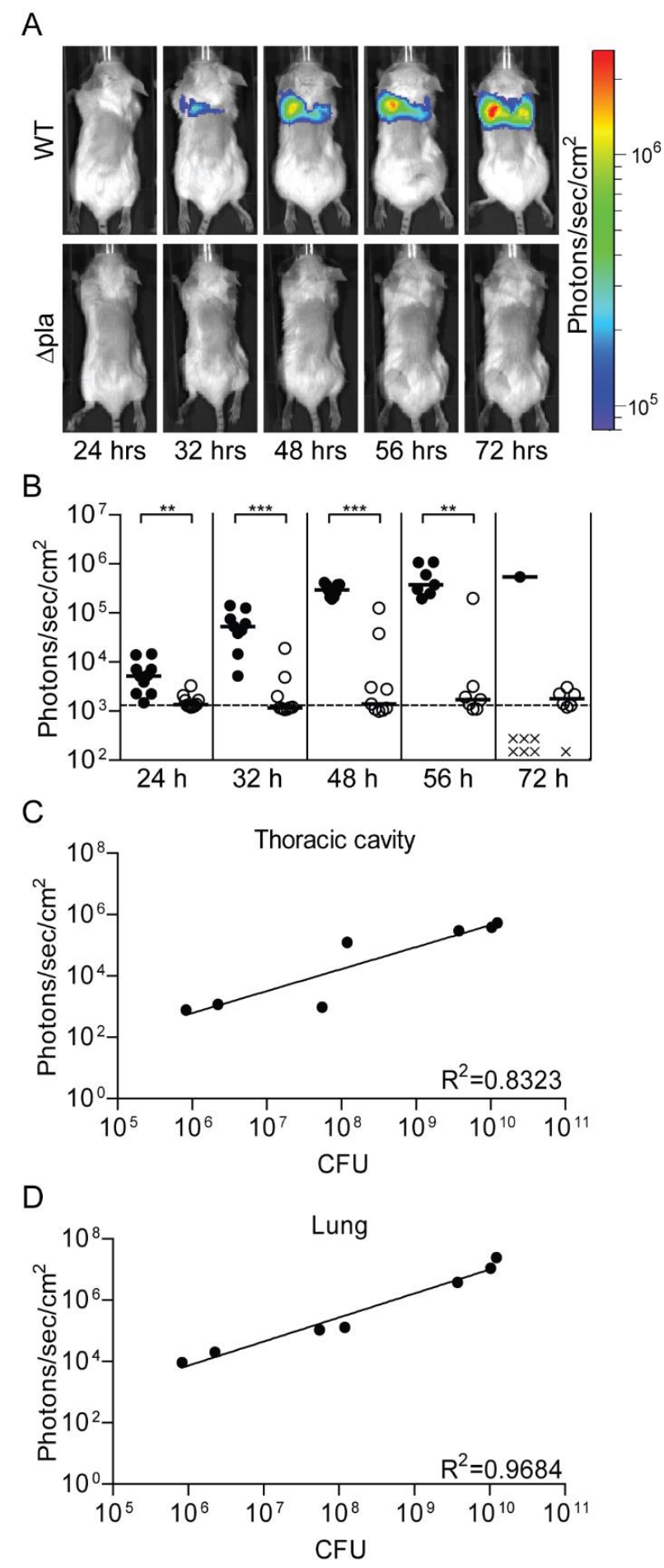
Figure 2-8. Progression of pneumonic infection.

Mice were infected with $5 \times 10^{4}-1 \times 10^{5}$ CFU of $Y$. pestis LuXPcyszK intranasally and imaged using an IVIS Spectrum. (A) Sequential images from representative animals. (B) For each animal, average bioluminescence was calculated for the thoracic cavity using the ROI tool in Living Image 3.2 software package. Black and white symbols represent animals infected with WT or $\Delta$ plaY. pestis, respectively. Dotted line represents the limit of detection based on images from uninfected animals. ${ }^{* *}=p<0.005,{ }^{* * *}=p<0.001$. At various time points, lungs were harvested from a subset of animals to determine bacterial loads (CFU) and compared to bioluminescence from the thoracic cavity (C) or from the lungs ex vivo (D). 


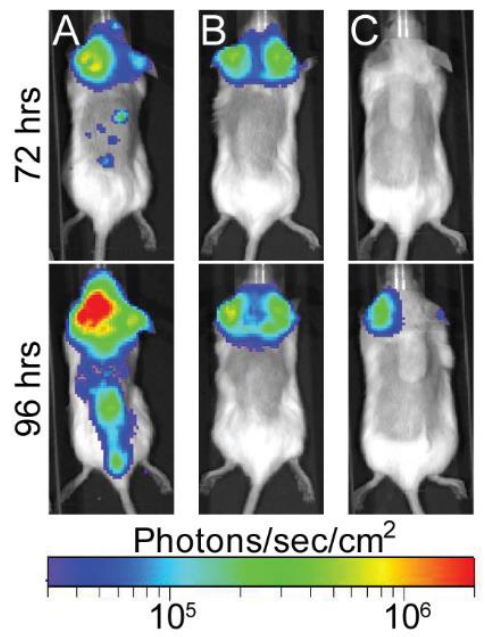

Figure 2-9. Extended imaging of animals intranasally infected with $\Delta$ pla.

$30 \%$ of animals infected intranasally with the $\Delta$ pla mutant in Figure 6 developed bioluminescence signal from regions corresponding to the head. A, B, and C represent three individual animals. Animal A also represents an example of a $\Delta$ pla infected animal that developed septicemic plague. 
$\underline{\text { Tables }}$

Table 2-1. Strains and plasmids used in this work

\begin{tabular}{|c|c|c|}
\hline Bacterial Strains & & \\
\hline MBLYP-001 & Y. pestis CO92; one passage from YP003-1 & [9] \\
\hline MBLYP-043 & MBLYP-001 with LuXPcysZK reporter & This work \\
\hline MBLYP-010 & Y. pestis CO92 $\Delta$ pla; one passage from YP102 & [176] \\
\hline MBLYP-045 & MBLYP-010 with LuXPcyszK reporter & This work \\
\hline YPA035 & MBLYP-001 pCD1(-) & This work \\
\hline YPA038 & YPA035 with LUXPEM7 reporter & This work \\
\hline YPA039 & YPA035 with LuXPtolc reporter & This work \\
\hline YPA040 & YPA035 with LuXPcyszK reporter & This work \\
\hline YPA047 & YPA035 $\Delta$ phoP & This work \\
\hline YPA073 & YPA047 with LUXPEM7 reporter & This work \\
\hline YPA048 & YPA047 with LuXPtolc reporter & This work \\
\hline YPA049 & YPA047 with LuXPcysZK reporter & This work \\
\hline YPA022 & YPA035 with pGEN-luxCDABE plasmid & This work \\
\hline Plasmids & & \\
\hline pGEN-IuxCDABE & Lux operon with EM7 promoter & [170] \\
\hline $\begin{array}{l}\text { pUC18r6k-mini- } \\
\text { Tn7(kanEW) }\end{array}$ & pUC18r6k-mini-Tn7 w/ modified Kan cassette & [171] \\
\hline pLOU027 & pUC18r6k-mini-Tn7(kanEW):: LUXPЕM7 & This work \\
\hline pLOU034 & pUC18r6k-mini-Tn7(kanEW):: LuXPtolc & This work \\
\hline pLOU037 & pUC18r6k-mini-Tn7(kanEW):: LuXPcyszk & This work \\
\hline pTNS2 & Tn7 transposase helper plasmid & [171] \\
\hline pSKIPPY & $\begin{array}{l}\text { pLH29 w/ Cat cassette replaced with Kan } \\
\text { cassette }\end{array}$ & [183] \\
\hline
\end{tabular}


CHAPTER 3:

\section{YERSINIA PESTIS REQUIRES HOST RAB1B FOR SURVIVAL IN MACROPHAGES ${ }^{2}$}

${ }^{2}$ Connor MG, Pulsifer AR, Price CT, Abu Kwaik Y, Lawrenz MB. Yersinia pestis Requires Host Rab1b for Survival in Macrophages. PLoS pathogens. 2015;11(10):e1005241. doi: 10.1371/journal.ppat.1005241. 


\section{Author Summary}

Yersinia pestis is the bacterial agent that causes the human disease known as plague. While often considered a historic disease, $Y$. pestis is endemic in rodent populations on several continents and the World Health Organization considers plague to be a reemerging disease. Much of the success of this pathogen comes from its ability to evade clearance by the innate immune system of its host. One weapon in the $Y$. pestis arsenal is its ability to resist killing when engulfed by macrophages. Upon invasion of macrophages, $Y$. pestis actively manipulates the cell to generate a protective vacuolar compartment, called the Yersinia containing vacuole (YCV) that allows the bacterium to evade the normal pathogen killing mechanisms of the macrophage. Here we demonstrate that the host protein Rab1b is recruited to the YCV and is required for $Y$. pestis to inhibit both the acidification and normal maturation of the phagosome to establish a protective niche within the cell. Rab1b is the first protein, either from the host or $Y$. pestis, shown to contribute to the biogenesis of the YCV. Furthermore, our data suggest a previously unknown impact of Rab1b recruitment in the phagosome maturation pathway.

\section{$\underline{\text { Overview }}$}

Yersinia pestis is a facultative intracellular pathogen that causes the disease known as plague. During infection of macrophages $Y$. pestis actively evades the normal phagosomal maturation pathway to establish a replicative niche within the cell. However, the mechanisms used by $Y$. pestis to subvert killing by the macrophage are unknown. Host Rab GTPases are central mediators of vesicular trafficking and are commonly targeted by bacterial pathogens to alter phagosome maturation and killing by macrophages. Here we demonstrate for the first time that host Rab1b is required for $Y$. pestis to effectively evade killing by macrophages. We also show that Rab1b is specifically recruited to the Yersinia containing vacuole (YCV) and that $Y$. pestis is unable to subvert YCV acidification when Rab1b expression is knocked down in macrophages. Furthermore, Rab1b knockdown also altered the frequency of association between the YCV with the lysosomal marker Lamp1, suggesting that Rab1b recruitment to the YCV directly inhibits phagosome maturation. Finally, we show that Rab1b knockdown also impacts the $\mathrm{pH}$ of the Legionella pneumophila containing vacuole, another pathogen that recruits Rab1b to its vacuole. 
Together these data identify a novel role for Rab1b in the subversion of phagosome maturation by intracellular pathogens and suggest that recruitment of Rab1b to the pathogen containing vacuole may be a conserved mechanism to control vacuole $\mathrm{pH}$.

$\underline{\text { Introduction }}$

Yersinia pestis is a facultative intracellular pathogen and causative agent of the disease known as plague. There have been three human plague pandemics in history; the most notable being the Black Death in the $14^{\text {th }}$ century $[1,5]$. Y. pestis can infect humans either through the bite of an infected flea or inhalation of contaminated aerosols. Flea inoculation can lead to the development of bubonic plague, a form of plague highlighted by bacterial dissemination to, and replication within, lymph nodes [1]. Inhalation of $Y$. pestis contaminated aerosols can result in rapid colonization of the lungs and development of pneumonic plague [1]. Both forms of plague are associated with acute disease progression and high mortality rates in the absence of timely antibiotic treatment. Furthermore, the potential for person-to-person transmission and use as a biological weapon in the absence of a vaccine highlights the risks associated with this pathogen [14].

During its natural life cycle, Y. pestis cycles between two different hosts, the mammal and the flea. The bacterium requires different virulence factors to colonize each host, and coordinates the expression of these factors accordingly [1]. Y. pestis has several well characterized antiphagocytic mammalian virulence factors, such as the Ysc type three secretion system (T3SS), secreted Yop effectors and the Caf1 capsule [1]. However, these virulence factors are down regulated in the flea vector and at the time of initial colonization of the mammalian host [1]. During this transitional period, $Y$. pestis is highly susceptible to phagocytosis by macrophages and neutrophils [51, 54]. Initial colonization of $Y$. pestis induces a rapid and early influx of neutrophils to the site of infection $[51,184]$. Upon phagocytosis by neutrophils, $Y$. pestis is readily killed by these professional phagocytes $[53,58,80]$. However, $Y$. pestis has demonstrated an increased ability to survive phagocytosis by monocytes and macrophages [ $51,54,57,59,82]$. Upon entry into the macrophage, $Y$. pestis actively circumvents the natural maturation of the phagolysosome by remodeling the phagosome into a hospitable replicative niche called the Yersinia containing 
vacuole (YCV) $[55,57,59,83,86]$. In vitro studies have highlighted three key characteristics of the biogenesis of the YCV. First, Y. pestis is able to actively inhibit the normal acidification of the phagosome and maintain a pH between 6.5 - 7.5 within the YCV throughout the course of intracellular infection [59]. Second, a significant portion of YCVs appear to become autophagosomes, which is highlighted by colocalization with LC3-II and the presence of double membranes surrounding the bacteria $[59,88]$. While the contribution of autophagy to intracellular survival is unclear, data indicates that autophagy contributes to the metabolism of intracellular bacteria [88, 89]. Finally, approximately eight hours after phagocytosis, the tight fitting vacuolar membrane of the YCV begins toexpand in size to form a spacious vacuolar compartment that can be observed by both light and electron microscopy $[54,59,62,86]$. Bacterial replication within the YCV usually coincides with spacious vacuole formation. Importantly, while the fate of $Y$. pestis in the macrophage has been characterized, the mechanisms used to generate the YCV and avoid macrophage killing have not been defined.

The ability of $Y$. pestis to survive within macrophages also appears to impact virulence of the bacterium. In vivo, intracellular $Y$. pestis are recovered from macrophages isolated from both infected nonhuman primates and rodents, but rarely from neutrophils isolated from the same animals $[49,58,84]$. Ye and colleagues further showed lower bacterial burdens in transgenic MaFIA mice selectively depleted of macrophage/dendritic cell populations, suggesting that macrophages are required to establish acute infection [52]. Y. pestis phoPQ mutants, which are defective for intracellular survival, are also attenuated during subcutaneous infection of BALB/C (75-fold change in $\mathrm{LD}_{50}$ ) and Swiss Webster mice (no change in $\mathrm{LD}_{50}$ but a significant delay in time to death for mutant infected animals) [56, 90]. Moreover, macrophages isolated from canines, a species that are relatively resistant to plague [91], are significantly more capable in killing Y. pestis than macrophages isolated from laboratory mice, a species highly susceptible to plague, suggesting that the ability of macrophages to kill $Y$. pestis may contribute to resistance to infection [62]. Together, these data highlight the importance of $Y$. pestis survival within the macrophage during pathogenesis 
Rab GTPases are the largest member of the Ras Superfamily of small guanine triphosphatases and are central mediators of vesicle trafficking within eukaryotic cells [102, 185]. These GTPases mediate vesicle trafficking by cycling through active GTP-bound and inactive GDPbound conformations $[102,185]$. When bound to GTP, the Rab protein integrates into specific vesicle membranes to mediate the trafficking of that vesicle through interactions with other trafficking proteins. Hydrolysis of the bound GTP to GDP results in extraction of the Rab from the membrane. While approximately 60 different Rab proteins have been identified, the contributions of only a few Rabs to specific vesicle trafficking steps have been experimentally described. For example, Rab5, Rab7, and Rab9 have been well studied as key mediators of important steps in the phagosome maturation process [94-96, 98, 100, 101]. Rab5 is recruited to the early endosome/phagosome and is required for phagocytosis [94-96, 98, 100, 101]. Following phagocytosis, Rab5 disassociates from the early endosome and Rab7 is recruited to the endosome to facilitate recruitment of Rab9 and subsequent fusion with the lysosome [94-96, 98, 100, 101]. A single disruption in the recruitment of a Rab protein to the maturing vesicle can stall and even terminate trafficking of that particular endocytic vesicle to its intended destination.

Due to the central role of Rab proteins for endosome sorting and phagosome maturation, many intracellular pathogens target Rab proteins to subvert these processes (see [102] for review). A classic example of Rab manipulation is seen in Mycobacterium infection of macrophages. $M$. avium and M. tuberculosis alter the normal distribution of Rab5 and Rab7 on their vacuole retention of Rab5 and exclusion of Rab7 - to inhibit phagosomal fusion with the lysosome and subsequent killing of the bacteria $[93,107,109,110,186,187]$. More recently, Rab1 has emerged as a common target required for the intracellular survival of many pathogens $[107,115,117,118$, 188-195]. Rab1 has two isoforms, Rab1a and Rab1b, which share $92 \%$ amino acid similarity and are thought to be functionally redundant $[196,197]$. Both isoforms have been shown to be involved in ER-to-Golgi trafficking [191, 198]. More recently Rab1a has been associated with proper endosome sorting during receptor mediated endocytosis and Rab1b has also been linked to autophagosome formation [192, 199-202]. Several pathogen containing vacuoles (PCVs) have been shown to associate with Rab1, and this recruitment is essential for subsequent survival of the 
pathogens contained within the PCV $[115,117,118,188-193,203]$. Coxiella burnetii requires Rab1 for the Coxiella replicative vacuole (CRV) to expand in both Chinese hamster ovary (CHO) and RAW264.7 macrophage cells [188]. This expansion is significantly hindered in the presence of a GTP restricted form of Rab1 [188]. Similarly, Anaplasma phagocytophilum also recruits Rab1 directly to the Anaplasma containing vacuole (APV) and it has been speculated that recruitment of Rab1 to the APV allows the bacteria to hijack endocytic trafficking [191]. Perhaps the best studied subversion of Rab1 by a pathogen comes from Legionella pneumophila. Rab1 has been shown to accumulate on the L. pneumophila containing vacuole (LCV) as early as $10 \mathrm{~min}$ after bacterial uptake and Rab1 knockdown has been shown to inhibit $L$. pneumophila intracellular replication $[115,118,189]$. Furthermore, several L. pneumophila secreted effectors have been identified that specifically target and modify Rab1 to alter its localization $[115,118,120,121,124,128,189,193$, $203,204]$. In contrast to the requirement of Rab1 for the survival of these intracellular pathogens that exist within vacuoles, Shigella flexneri, which replicates in the host cytoplasm, is hindered by Rab1 [190]. Inactivation of Rab1 by $S$. flexneri is critical for bacterial survival and is mediated by the VirA/EspG secreted effector family [190]. Together, these studies suggest a distinct role for host Rab1 GTPases for intracellular survival of pathogens that replicate within vacuolar compartments.

Since Rab1 appears to be targeted by several pathogens that reside within vacuoles in order to survive intracellularly, we investigated the role of Rab1 in the survival of $Y$. pestis within macrophages. We demonstrate that siRNA knockdown of Rab1b in macrophages infected with $Y$. pestis significantly increases YCV acidification and association with the lysosomal marker Lamp1, resulting in decreased intracellular survival of $Y$. pestis. Furthermore, we show Rab1b is recruited to the YCV, suggesting a direct interaction with Rab1b is required for proper YCV maturation. Importantly, Rab1b is the first host protein to be identified that is required by $Y$. pestis to alter phagosome maturation and YCV acidification and impact the ability of this pathogen to survive within the eukaryotic cell. Finally, we also demonstrate for the first time that Rab1b recruitment to the L. pneumophila containing vacuole also impacts vacuole $\mathrm{pH}$, suggesting a conserved mechanism for the recruitment of Rab1b to pathogen containing vacuoles. 
Results:

\section{$\underline{\text { Rab1b is required for } Y \text {. pestis survival in macrophages }}$}

Since $Y$. pestis exists within a vacuolar compartment within macrophages [54, 55, 57], and Rab1 has been linked to survival of several other intracellular pathogens that exist within vacuoles $[117,118,188,189,191,192,194]$, we sought to determine if Rab1 is required for $Y$. pestis intracellular survival. Toward this goal, we initially screened whether either isoform of Rab1 is required for $Y$. pestis to survive in macrophages. RAW264.7 macrophages were transfected with either Rab1a or Rab1b specific siRNAs (pool of 3 siRNAs targeting each gene). $48 \mathrm{~h}$ after transfection, macrophages were infected with Y. pestis CO92 $\mathrm{pCD} 1^{(-)}$LuXPtolc, which contains a bioluminescent bioreporter to monitor $Y$. pestis numbers [151]. Extracellular bacteria were killed with gentamicin, and intracellular bacterial survival was monitored via bioluminescent signal (Fig 31A). While no change in $Y$. pestis bioluminescence was observed in Rab1a siRNA treated cells compared to scrambled siRNA treated controls, we observed a significant decrease in bioluminescence in Rab1b siRNA treated cells, indicating that Rab1b, but not Rab1a, is required for $Y$. pestis survival within macrophages.

To confirm Rab1b is required for Y. pestis intracellular survival, RAW264.7 macrophages were transfected with a single Rab1b siRNA optimized for Rab1b knockdown and cell viability (Fig 3-S1) and infected with Y. pestis CO92 pCD1(-) LuXPtolC $48 \mathrm{~h}$ post-transfection. As a positive control, we also infected macrophages transfected with Cop $\beta 1$ siRNA. Cop $\beta 1$ is a component of the cotamer complex and has been shown to alter both invasion and survival of other intracellular pathogens [141, 205]. As expected, Cop $\beta 1$ knockdown resulted in a significant decrease in intracellular Y. pestis CO92 pCD1(-) LuXPtolc bioluminescence at $10 \mathrm{~h}$ post-infection as compared to scramble siRNA treated cells (Fig 3-1B; $p \leq 0.0001)$. Rab1b knockdown also resulted in a significant decrease in bioluminescent signal; Y. pestis CO92 pCD1(-) LuXPtolc bioluminescence was $~ 50 \%$ less in Rab1b siRNA treated cells (Fig 1B; $p \leq 0.0001)$. To confirm that Y. pestis CO92 pCD1(-) LuXPtolc bioluminescence accurately represents viable intracellular bacteria, cells were lysed and bacterial numbers were determined by conventional serial dilution enumeration (Fig 3-1C). Conventional enumeration supported our bioluminescent data and demonstrated a significant 
decrease in viable intracellular colony forming units (CFU) in Rab1b siRNA treated cells $(p \leq 0.001)$. No differences in survival were observed if bacteria were grown at $37^{\circ} \mathrm{C}$ prior to infection (Fig 3S2). Importantly, the direct correlation between bioluminescent signal and bacterial enumeration support the use of bioluminescent data to monitor intracellular $Y$. pestis numbers.

To confirm that the pCD1 encoded Ysc type three secretion system (T3SS) does not impact Rab1b mediated $Y$. pestis survival, Rab1b transfected cells were also infected with $Y$. pestis KIM D-19 LuXPtolc, which contains the PCD1 plasmid and the Ysc T3SS, and bacterial survival was monitored by bioluminescence and conventional bacterial enumeration (Fig 3-1E and F). As observed for $Y$. pestis CO92 pCD1(-) LuXPtolc, we observed an $\sim 50 \%$ decrease in Y. pestis KIM D19 LuXPtolC survival in Rab1b siRNA treated cells $(p \leq 0.001)$. We also monitored $Y$. pestis intracellular bioluminescence temporally over the course of the infection to determine how early during infection $Y$. pestis intracellular survival was impacted by Rab1b knockdown. This analysis revealed that intracellular bacterial numbers for both strains were significantly decreased in Rab1b treated cells as early as $2 \mathrm{~h}$ post-infection, which is the earliest time point we can monitor after gentamicin removal (Fig 3-1D and G; $p \leq 0.001$ ). Finally, to determine if the Rab1b impact on intracellular survival is conserved in the Yersinia genus, transfected macrophages were infected with Y. pseudotuberculosis and Y. enterocolitica. As observed for Y. pestis, both enteric species were attenuated in survival when Rab1b was knocked down (Fig 3-S1). Together these data demonstrate that Rab1b is required for Yersinia intracellular survival, which is independent of the Ysc T3SS, and bacterial survival is impacted by Rab1b very early during the infection process.

\section{$\underline{\text { Rab1b is not required for } Y \text {. pestis invasion of macrophages }}$}

We observed a difference in Y. pestis intracellular numbers in Rab1b siRNA treated cells within $2 \mathrm{~h}$ of macrophage infection (Fig 3-1D and G). The difference in recovered bacteria at this early time point could be due to an inability of $Y$. pestis to avoid phaogolysomal killing in the absence of Rab1b. However, Rab1b may also be required for efficient phagocytosis and the difference in $Y$. pestis numbers at $2 \mathrm{~h}$ post-infection could be a result of less bacteria gaining entry into the macrophages prior to gentamicin treatment. Because phagolysosome fusion and bacterial killing can occur within 120 minutes of phagocytosis [94, 102], we could not rely on the conventional 
gentamicin protection assay, which requires a $1 \mathrm{~h}$ incubation period, to differentiate between invasion and bacterial killing in Rab1b siRNA treated cells. Therefore, we used a differential staining procedure to specifically label extracellular $Y$. pestis and determine if Rab1b knockdown impacted Y. pestis invasion of macrophages by confocal microscopy. Rab1b siRNA transfected RAW264.7

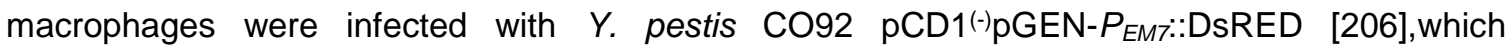
constitutively expresses the DsRED fluorescent protein. At 20 and 80 min post-infection, cells and total bacteria were fixed with paraformaldehyde. Extracellular bacteria were then specifically labeled with anti-Y. pestis polyclonal antibody and Alexa Fluor 488 anti-rabbit secondary antibody (Fig 3-2A). As a positive control, macrophages were treated with Cop $\beta 1$ siRNA, which has been previously shown to be required for efficient phagocytosis [141]. As expected, cells treated with Cop $\beta 1$ had significantly less intracellular $Y$. pestis than scrambled siRNA treated macrophages at both 20 and 80 min post-infection (Fig 3-2B and C; $p \leq 0.001$ ). Conversely, we observed no difference in the proportion of intracellular $Y$. pestis in Rab1b siRNA treated cells compared to scrambled siRNA treated cells. These data demonstrate that Rab1b is not required for phagocytosis of $Y$. pestis and suggest that the differences in intracellular bacterial numbers in Rab1b siRNA treated cells is due to a decreased ability of $Y$. pestis to avoid macrophage killing in the absence of Rab1b.

\section{Rab1b is required for Y. pestis to avoid YCV acidification.}

A hallmark characteristic of $Y$. pestis infection of the macrophage is that the bacterium is able to rapidly subvert normal acidification of the YCV [59]. Because acidification is one of the earliest steps in phagosome maturation and is required for both efficient lysosomal fusion and degradation of phagolysosomal contents [94], we next investigated whether Rab1b is required for $Y$. pestis to avoid YCV acidification. RAW264.7 macrophages were transfected with Rab1b siRNA and then treated with Lysotracker Red DND-99 prior to infection with Y. pestis CO92 pCD1(-) pGEN222, which constitutively expresses EGFP. Lysotracker Red DND-99 fluorescence is $\mathrm{pH}$ dependent (fluoresces below $\mathrm{pH}$ 5.5), and therefore, allows for identification of acidified vacuoles. As Y. pestis inhibition of YCV acidification is an active process, untransfected cells were infected with paraformaldehyde killed Y. pestis CO92 pCD1(-) pGEN222 to serve as a positive control for YCV 
acidification. As previously reported for untransfected macrophages [59], Y. pestis CO92 pCD1(-) pGEN222 efficiently avoided YCV acidification in scramble siRNA treated macrophages, with $<25 \%$ of $Y$. pestis found within acidified vacuoles by 80 min post-infection (Fig 3-3). This was significantly lower than paraformaldehyde killed $Y$. pestis, which were already within acidified vacuoles $>80 \%$ of the time by 20 min post-infection ( $p \leq 0.01$ ). The ability of $Y$. pestis to inhibit YCV acidification was greatly attenuated in Rab1b knocked down cells, where $~ 70 \%$ of the bacteria were observed within acidified vacuoles within 20 min post-infection ( $p \leq 0.01$ ). Furthermore, Y. pestis remained within acidified vacuoles in Rab1b siRNA treated macrophages at 80 min post-infection. These data demonstrate that $Y$. pestis requires the host Rab1b GTPase to inhibit or avoid YCV acidification.

\section{Rab1b is necessary for $Y$. pestis to avoid fusion with the lysosome.}

Acidification of the phagosome precedes or coincides with fusion to lysosomes and degradation of foreign particles such as bacteria [94]. As Rab1b knockdown resulted in increased acidification of the YCV, we next determined if Rab1b is required for $Y$. pestis to avoid fusion with lysosomes. RAW264.7 macrophages were transfected with Rab1b siRNA and infected with live or

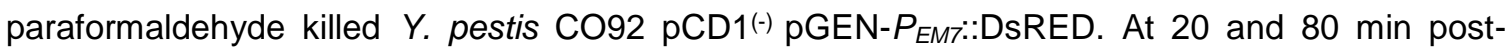
infection, cells were washed, fixed with paraformaldehyde, and stained with anti-Lamp1 antibody, a marker for lysosomal fusion (Fig 3-4A). In scrambled siRNA treated cells, we observed minimal association of live $Y$. pestis with Lamp1 (<25\%) at 20 and 80 min post-infection, indicating limited association between the YCV and lysosomes at these time points (Fig 3-4B and C). As observed for YCV acidification, there was a significant increase in the association between Lamp1 and paraformaldehyde killed $Y$. pestis ( $>60 \%$ ), supporting an active avoidance of lysosomal fusion by Y. pestis during macrophage infection (Fig 3-4B and C; p 50.001). Rab1b knockdown also significantly altered Lamp1 association with the YCV compared to scramble siRNA (Fig 3-4B and C; $p \leq 0.001$ and $p \leq 0.01$, respectively). At 20 min post-infection, Lamp1 associated with $\sim 55 \%$ of YCVs in Rab1b siRNA treated cells, and was maintained at this elevated level at 80 min postinfection. These data indicate that Rab1b is required not only for $Y$. pestis to inhibit $Y C V$ acidification but also to avoid lysosomal fusion. Importantly, the $~ 2$-fold increase in association with Lamp1 
directly correlates to a similar 2-fold decrease in $Y$. pestis survival in Rab1b siRNA treated macrophages (Fig 3-1).

\section{$\underline{\text { Rab1b is not required for early Y. pestis association with LC3 }}$}

Autophagy has been linked to both $Y$. pestis and Y. pseudotuberculosis intracellular infection and may be required for sustained bacterial metabolism within cells $[59,88]$. Furthermore, studies have shown a recruitment of LC3, a marker for autophagosomes, to the YCV during $Y$. pseudotuberculosis infection of HeLa cells and BMDMs [88, 89]. Recently, Huang and colleagues demonstrated a potential role for Rab1b in autophagy and intracellular survival of Salmonella enterica Typhimurium [192]. Given the link of Rab1b to autophagy and autophagy to Yersinia intracellular infection, we next investigated if knockdown of Rab1b impacted early association of LC3 to the YCV during macrophage infection. RAW264.7 macrophages were transfected with Rab1b siRNA and infected with live or paraformaldehyde killed Y. pestis CO92 pCD1(-) pGEN$P_{E M 7:: D s R E D} 20$ and 80 min post-infection cells were washed, fixed with paraformaldehyde, and stained with anti-LC3 antibody (Fig 3-5A). In contrast to reported infection of epithelial cells with $Y$. pseudotuberculosis [89], we observed a very low incidence in the association between live or killed Y. pestis with LC3 during early stages of macrophage infection (Fig 3-5B and C) and this association was not significantly altered in Rab1b siRNA treated cells ( $20 \%$ association in all samples). These data support previous data that LC3 association with the YCV is lower in macrophages than epithelial cells $[88,89]$ and demonstrate that Rab1b knockdown does not alter YCV-LC3 association during the early stages of $Y$. pestis infection when we observe changes in YCV maturation and intracellular survival of the bacteria.

\section{Rab1b is recruited to the YCV during macrophage infection.}

Rab GTPases mediate vesicular trafficking through direct interactions with vesicle membranes (see $[102,185]$ for review). Therefore, we next sought to determine whether Rab1b is recruited to the YCV during $Y$. pestis infection. Because Rab interactions with membranes are transient, we transfected RAW264.7 macrophages with a GFP-labelled, constitutively active form of Rab1b [eGFP-Rab1b(CA)]. eGFP-Rab1b(CA) contains a mutation in the GTP binding domain that inhibits 
the hydrolysis of GTP, resulting in retention of the protein in the membrane in which the Rab GTPase is recruited [118, 191, 207, 208]. Twenty-four hours after transfection, macrophages were infected with either live or PFA killed $Y$. pestis CO92 pCD1 ${ }^{(-)}$pGEN::mCherry or E. coli $\mathrm{K} 12$ pGEN::mCherry, which constitutively express the mCherry fluorescent protein. Cells were washed and fixed with paraformaldehyde at 20 and 80 min post-infection and analyzed by confocal microscopy to determine localization of eGFP-Rab1b(CA) (Fig 3-6). Less than $25 \%$ of E. coli or PFA killed Y. pestis, which traffic to acidified vacuoles, colocalized with eGFP-Rab1b(CA) at 20 min post-infection (Fig 3-6B). Furthermore, we observed no significant change in colocalization at 80 min post-infection. However, in cells infected with live $Y$. pestis, we observed a significant increase in eGFP-Rab1b(CA) localization to the YCV at both time points (Fig 3-6B and C; 57\%; $p \leq 0.05)$. These data demonstrate that while Rab1b is minimally associated with phagosomes containing E. coli or dead Y. pestis, the GTPase is associated with the YCV containing live Y. pestis at a significantly higher frequency, suggesting that Rab1b recruitment or retention to the YCV specifically contributes to $Y$. pestis survival.

\section{Disruption of the secretory pathway does not alter Y. pestis survival or inhibition of YCV acidification}

Rab1b has an important role in mediating ER-to-Golgi trafficking [209, 210]. While Rab1b appears to be directly recruited to the YCV, it is also possible that the effect of Rab1b knockdown on $Y$. pestis survival is due to changes in Golgi trafficking. To determine if Golgi trafficking, specifically secretory trafficking, is required for $Y$. pestis to inhibit YCV acidification, we treated RAW264.7 macrophages with Brefeldin A (BFA), which blocks Golgi trafficking independent of Rab1b by targeting Arf1. BFA-treated macrophages were infected with $Y$. pestis CO92 pCD1(-) LuXPtolc for 20 min, extracellular bacteria were killed with gentamicin, and intracellular bacteria bioluminescence was monitored at 2 and $10 \mathrm{~h}$ post infection (Fig 3-7A and B, respectively). At both time points there was no significant difference in the survival of $Y$. pestis between untreated macrophages or cells treated with increasing concentrations of BFA. Macrophages treated with 10 $\mu \mathrm{M}$ BFA were also incubated with Lysotracker Red DND-99 and subsequently infected with $Y$. pestis CO92 $\mathrm{pCD1}^{(-)}$pGEN222 to determine if inhibition of the secretory pathway altered YCV 
acidification. As a control, a separate group of cells were infected with paraformaldehyde killed $Y$. pestis CO92 $\mathrm{pCD} 1{ }^{(-)}$pGEN222. In agreement with the intracellular bacterial survival, there was no significant difference between YCV acidification in BFA-treated macrophages at 20 or 80 min postinfection compared to untreated cells (Fig 3-7C and D). Furthermore, BFA treatment did not alter the acidification of phagosomes containing paraformaldehyde killed $Y$. pestis. Together these data demonstrate that $Y$. pestis avoidance of the phagolysosome is independent of retrograde endocytic trafficking and suggests that Rab1b impacts YCV maturation independent of its function in Golgi trafficking.

\section{$\underline{\text { Rab1b inhibition results in increased acidification of the Legionella containing vacuole }}$}

Previous studies with L. pneumophila demonstrate the cyclic recruitment and release of Rab1b on the LCV within 2 hours post-infection [117]. The release of Rab1b from the nascent LCV coincides with the transition of the LCV from a neutral to acidic $\mathrm{pH}[211,212]$. Given that $Y$. pestis recruits Rab1b to the YCV to prevent vacuole acidification, we hypothesized that $L$. pneumophila recruitment of Rab1b may also result in arrest of LCV acidification. To test this hypothesis, we transfected RAW264.7 macrophage cells with siRNA targeting Rab1b and treated transfected cells with Lysotracker Red DND-99 prior to infection with L. pneumophila to monitor LCV acidification. As previously reported, we observed that the majority of LCVs did not colocalize with Lysotracker in scramble siRNA treated macrophage (only $30 \%$ of L. pneumophila was found in acidified compartments by 80 min post-infection; Fig 3-8). In contrast, we observed a significant increase in Lysotracker colocalization in macrophages treated with siRNA targeting Rab1b at both 20 and 80 min post-infection (Fig 3-8B and $\mathrm{C} ; \mathrm{p} \leq 0.01$ and $\mathrm{P} \leq 0.001$, respectively). These data demonstrate that like Y. pestis, L. pneumophila requires Rab1b to inhibit LCV acidification during early stages of macrophage infection.

\section{Discussion}

Rab proteins are central mediators in vesicular trafficking within the cell. As such, intracellular pathogens often target these GTPases to subvert the normal phagosome maturation pathway and survive within host cells (see $[102,103]$ for reviews). Rab1 was one of the first identified members 
of this family and has been extensively studied for its role in Golgi trafficking in yeast, Drosophila, and mammalian cells (see $[210,213,214]$ for reviews). More recently, both isoforms of Rab1 have been linked to intracellular infection by several pathogens. Chlamydial species [194], L. pneumophila [118, 189], A. phagocytophilum[191], Coxiella burneti[188], and S. enterica Typhimurium[192] have been shown to recruit Rab1 to the PCV. Furthermore, inhibition of Rab1 by either RNAi or expression of dominant negative Rab1 constructs indicate that Rab1 function is required for the survival/growth of L. pneumophila [189], C. burnetii [188], S. enterica Typhimurium [133, 192], and Brucella melitensis [138]. Our data demonstrate for the first time that $Y$. pestis also belongs to this group. Specifically, we have demonstrated that $Y$. pestis recruits Rab1b to the YCV during infection of macrophages and that this GTPase is required for intracellular survival. Interestingly, Rab1 has only been shown to be required for the survival of pathogens that exist within vacuolar compartments, suggesting a role(s) for Rab1 in subverting normal phagosome maturation and generation of a protective PCV. In fact, functional Rab1 has been shown to be detrimental to the survival of the cytoplasmic pathogen Shigella flexnerii through its interaction with the autophagy system within the host cell [190]. However, S. flexnerii has also evolved to target Rab1, through the VirG secreted effector protein, and inactivate the GTPase to inhibit macroautophagy during infection [190].

While Rab1 has been linked to the survival of several intracellular pathogens, the role Rab1 plays in the maturation of individual PCVs is less well understood. In C. burnetii, Rab1 has been shown to be required for the massive expansion of the Coxiella replicative vacuole (CRV) [188]. This requires the acquisition of new membrane in order for the CRV to grow, and Rab1 recruitment to the vacuole may mediate the interception of vesicles (and their membranes) from the secretory pathway. This hypothesis is supported by studies showing that treatment with BFA, which independently inhibits the secretory pathway, also inhibits the expansion of the CRV [188]. Studies from A. phagocytophilum and Chlamydial species, which also form a large replicative vacuole, also suggest that Rab1 recruitment is important for formation of a spacious vacuolar compartment [191, 194]. Therefore, a common goal of bacteria that recruit Rab1 to their PCV may be to subvert the secretory pathway in order to remodel the PCV. Furthermore, Rab1b has also been linked to 
autophagy [192], which is also associated with the replication of both $C$. burnetii and $A$. phagocytophilum $[215,216]$. It is possible that in addition to the secretory pathway, Rab1 recruitment may also contribute to the recruitment of autophagsomal membranes to these PCV, though this has yet to be demonstrated. Since the YCV also expands late during infection (though not to the degree of these former pathogens) to form a spacious vacuole $[54,59,62]$, it is possible that Rab1b may contribute to YCV expansion. However, we have not observed changes in spacious YCV formation in Rab1b siRNA treated macrophages. Furthermore, our data also suggest that early association with the autophagosome marker LC3 does not appear to protect YCV from acidification, as we observed no difference in YCV-LC3 association in Rab1b siRNA treated cells. More importantly, our data with $Y$. pestis reveal a potential new benefit of Rab1 recruitment to the PCV, which is to avoid phagosomal acidification and subsequent fusion to the lysosome. While it is currently unclear how Rab1b inhibits YCV acidification, it appears to be independent from its contributions to the secretory pathway, as BFA treatment did not result in similar changes to YCV acidification. Importantly, while knockdown of Rab1B does not alter the expression of Rab 5, 7 or 9, which are required for phagosome maturation (Fig 3-S1A), it is possible that recruitment and retention of Rab1b to the early phagosome inhibits interactions with these Rabs (and/or Rab effector proteins) to inhibit normal phagosome maturation. Rab1 has also been linked to endosomal sorting through direct interactions with the kinesin Kifc1, which in turn affects directional vesicular motility within the cell $[201,202]$. Thus, Rab1b recruitment may alter early sorting of the YCV to avoid acidification and lysosomal fusion. Studies to better characterize the early YCV, including differences in Rab composition and vATPase recruitment as compared to the normal phagosome are ongoing and will provide further insight into these mechanisms. Rab1 recruitment to the YCV also occurs significantly earlier than reported for $C$. burnetii ( $\leq 20$ min vs. $>12 \mathrm{~h}$, respectively) [188], suggesting that timing of recruitment may indicate which function, inhibition of phagosome maturation or membrane acquisition, is contributing to pathogenesis of various pathogens. It should be noted that $C$. burnetii requires passage through an acidified vacuole to induce the expression of important virulence factors and subsequent intracellular survival [217]. Therefore, our observations that early acquisition of Rab1 inhibits PCV acidification may explain why Rab1 recruitment is 
delayed during C. burnetii infection. In contrast to C. burnetii, L. pneumophila, which inhibits LCV acidification early during infection[211, 212, 218], recruits Rab1 in a similar time frame as seen during $Y$. pestis infection (within $10 \mathrm{~min}$ ) [118]. In support of our hypothesis that early recruitment of Rab1b is a mechanism for pathogens to inhibit phagosome acidification, we demonstrated that knockdown of Rab1b decreased the ability of L. pneumophila to inhibit LCV acidification (Fig 3-8). Interestingly, L. pneumophila appears to control both recruitment and later release of Rab1 from the LCV (discussed below). The timing of Rab1 modification by L. pneumophila coincides with a transition from a neutral to an acidic LCV [211, 212], suggesting that Rab1 inhibition of acidification may be an active process that is reversible upon removal of Rab1 from the vacuolar membrane.

Phagosome acidification has been shown to be a key step in phagosome maturation. Acidification of the phagosome is believed to work in concert with Rab5, Rab7 and Rab9 to mediate phagosome maturation and ultimately fusion with lysosomes [94, 95]. Initially, the early phagosome, highlighted by association with Rab5, is slightly acidic ( pH 6.0). As the phagosome matures, the $\mathrm{pH}$ decreases and Rab7 replaces Rab5 on the phagosome. Rab7 subsequently recruits more vATPase complexes, resulting in further acidification of the phagosome and recruitment of Rab9. By the time Rab9 mediates lysosomal fusion, the $\mathrm{pH}$ of the phagosome is approaching 4.0, which is the optimal $\mathrm{pH}$ to activate hydrolases and proteases delivered to the phagosome by the lysosome. Several lines of evidence indicate that acidification of the phagosome is required in order for efficient lysosomal fusion and function to occur [100, 101, 219-221], which suggest that inhibition of acidification could influence proper lysosomal fusion to the PCV. In line with these hypotheses, we observed a direct correlation between increased YCV acidification with increased Lamp1 association, and subsequent decreased Y. pestis survival, in Rab1b siRNA treated cells. This direct correlation makes it difficult to separate the impact of acidification directly on $Y$. pestis survival (acidic killing) from lysosomal fusion, but further supports the importance of inhibiting YCV acidification as mechanism for Y. pestis intracellular survival [59].

While Rab1 is important for the intracellular survival of several pathogens, bacterial virulence factors that target Rab1 have only been identified for Chlamydia [194] and L. pneumophila $[116,117,119,122,125,129,193,222,223]$. In the case of $L$. pneumophila, multiple Dot/lcm 
secreted factors have been shown to target Rab1 and modify the protein to manipulate localization to the LCV; cycling the host Rab1 between active (anchored to the LCV) and inactive states. The effectors DrrA/SidM, SidD and LepB work in concert to first recruit Rab1 to LCV, and then later remove it $[113,115,120,121,193]$. L. pneumophila also manipulates Rab1 independent of recruitment to the LCV through the action of SidC/SdcA, LidA and AnkX $[116,127,129,223]$. The redundancy in Rab1 targeting proteins indicates that Rab1 manipulation by L. pneumophila is extremely important for the intracellular survival of this pathogen. For $Y$. pestis, we have yet to define the virulence factors that mediate Rab1b recruitment to the YCV. However, we have shown that $Y$. pestis does not require the pCD1 plasmid (including the Ysc T3SS) or the high pathogenicity island (pgm locus) to recruit Rab1b and inhibit YCV acidification. These findings are in agreement with previous work that has shown both of these genetic elements are dispensable for intracellular survival [49, 53-55]. Therefore, virulence factors encoded elsewhere in the genome are mediating both Rab1b interactions and intracellular survival. While the PhoPQ two component regulator has been shown to contribute to intracellular survival, likely through the regulation of other genes [56, $83,86,90]$, we speculate that these genes do not regulate survival through Rab1b because phoPQ mutants still inhibit YCV acidification during infection [86]. However, defining Rab1b recruitment to the phoPQ mutant YCV is needed to confirm this hypothesis. Studies to specifically identify $Y$. pestis factors involved in Rab1b recruitment to the YCV are ongoing.

In summary, we have shown here for the first time that recruitment of Rab1b to the PCV directly correlates to the ability of a pathogen to inhibit acidification of the vacuole. These findings indicate a novel function for Rab1b in inhibiting phagosome maturation and suggest that other pathogens may use a similar strategy to modify the maturation of the PCV. Furthermore, in the context of $Y$. pestis infection, Rab1b is the first factor, either host or bacterial, identified that directly impacts acidification of the YCV. Future studies to define how Rab1b impacts phagosome acidification and to identify additional host factors that contribute YCV biogenesis will be important for us to understand how this pathogen evades killing by macrophages. 


\section{Materials and Methods}

Bacterial strains, plasmids, and macrophages. All bacterial strains used in this study are listed in Table 3-S1 in the Supplementary Information. Y. pestis CO92 [9] pCD1(-) and KIM D-19(pgm $\left.{ }^{(-)}\right)(B E I$ Resources) were cultivated at $26^{\circ} \mathrm{C}$ in Brain Heart Infusion (BHI) broth (Difco). When needed, carbenicillin was used at $50 \mu \mathrm{g} / \mathrm{mL}$. Bioluminescent derivatives were generated using the LuXPtolc bioreporter as described previously [151]. To generate fluorescent bacterial strains, Y. pestis and

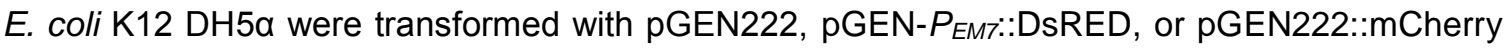
[206]. E. coli was cultivated at $37^{\circ} \mathrm{C}$ in Luria-Bertani (LB) broth (Difco) supplemented with $50 \mu \mathrm{g} / \mathrm{mL}$ carbenicillin. L. pneumophila AA100, a clinical isolate containing pMIP-GFP, was grown on BCYE agar plates for 3 days at $37^{\circ} \mathrm{C}$ prior to macrophage infection [224-226]. The pGEN222::mCherry plasmid was generated by replacing the EGFP gene from pGEN222 with the mCherry gene using Gibson Cloning [227]. Constitutive active EGFP-Rab1b was generated by site directed mutagenesis of pEGFP-Rab1b [108] using primers 5'- TGG AAC GGT TCC GGA C-3' and 5'- GGC CCG CTG TGT CC-3' to mutate the Glutamine at residue 67 to a Leucine as previously described [108]. RAW264.7 macrophages were obtained from ATCC and cultured in DMEM, $100 \mathrm{mM}$ glucose $+10 \%$ FBS (Hyclone).

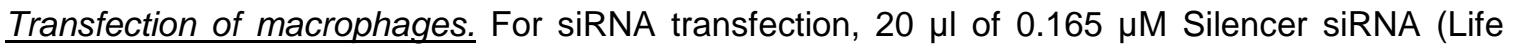
Technologies) diluted in Opti-MEM (Life Technologies) was mixed with $10 \mu \mathrm{l}$ of $0.03 \%(\mathrm{v} / \mathrm{v})$ Lipofectamine RNAiMax/Opti-MEM (Life Technologies) as described by the manufacturer. $30 \mu \mathrm{l}$ of the siRNA-Lipofectamine complex was added to each well of a white flat-bottom 96-well plate (Greiner), incubated at room temperature for $10 \mathrm{~min}$, and then $1 \times 10^{4}$ RAW264.7 macrophages suspended in $80 \mu \mathrm{l}$ of DMEM+10\% FBS were added. Cells were incubated for $48 \mathrm{~h}$ at $37^{\circ} \mathrm{C}$ with $5 \% \mathrm{CO}_{2}$. For 24-well plates used for microscopy, all reagents were increased by 4 -fold. For plasmid transfection, $4 \mu \mathrm{g}$ of plasmid was transfected into $4.4 \times 10^{5}$ RAW264.7 macrophages using Lipofectamine 2000 (Life Technologies) or $0.5 \mu \mathrm{g}$ of plasmid with JetPrime (Polyplus) as described by the manufacturers. Luminescence was monitored with a Synergy 4 plate reader (BioTek) (1 sec read with sensitivity set at 150$)$. 
Bacterial infection of macrophages. Macrophages were infected with $Y$. pestis strains as previously described [57, 151]. Briefly, bacteria were grown at $26^{\circ} \mathrm{C}$ in $\mathrm{BHI}$, washed in PBS, and diluted appropriately in prewarmed DMEM+10\%FBS. Bacteria were added to macrophages and the infection was synchronized by centrifugation. After $20 \mathrm{~min}$, extracellular bacteria were killed with gentamicin $(16 \mu \mathrm{g} / \mathrm{mL})$. One hour after gentamicin treatment, the medium was replaced with DMEM $+10 \%$ FBS containing $2 \mu \mathrm{g} / \mathrm{mL}$ gentamicin. Intracellular $Y$. pestis numbers were determined by bioluminescence using a Synergy HT plate reader (Biotek) or conventional bacterial enumeration as described previously [151]. For L. pneumophila, bacteria were swabbed directly from plates and diluted appropriately in prewarmed DMEM $+10 \%$ FBS. Bacteria were added to macrophages and the infection was synchronized by centrifugation. At 20 minutes and 80 minutes post-infection cell monolayers were washed three times with PBS and fixed as described below [225, 226]. All MOls were confirmed by conventional enumeration of the inoculum at the time of infection. For vacuole acidification experiments, $75 \mathrm{nM}$ Lysotracker Red DND-99 (Life Technologies) was added to the cells $1 \mathrm{~h}$ prior to fixation. Brefeldin A (Sigma) was added to cells $2 \mathrm{~h}$ prior to $Y$. pestis infection and maintained throughout the infection.

Immunofluorescent staining and confocal microscopy. For confocal microscopy, cells were fixed to coverslips with $4 \%$ paraformaldehyde for $30 \mathrm{~min}$. For indirect immunofluorescent staining, fixed cells were blocked with $3 \%$ BSA overnight and incubated with rabbit anti- $Y$. pestis serum $(1: 1,000)$, anti-Lamp1 (0.8ug/ul; Abcam ab24170), or anti-MAP-LC3a/ß (1:200; Santa Cruz sc-16756) antibodies for $1 \mathrm{~h}$. Unbound primary antibodies were removed by washing and anti-rabbit Alexa Fluor 488 secondary antibody (1:4000; Life Technologies) was added for $1 \mathrm{~h}$. All coverslips were mounted with Prolong Gold with DAPI (Life Technologies) and imaged on an Olympus FV100 laser or Zeiss LSM 710 laser confocal microscope. Colocalization of Lysotracker Red DND-99 or proteins to the YCV was determined using the Coloc function in the Imaris image analysis software (BitPlane).

Statistics. All data are shown as mean and standard deviation of three to six biological replicates and each experiment was repeated three times to confirm the phenotypes. For microscopy, at least 
50 vacuoles per biological replicate were analyzed. $p$-values were calculated by one-way ANOVA (or t-test for L. pneumophila experiments) using GraphPad Prism software. 

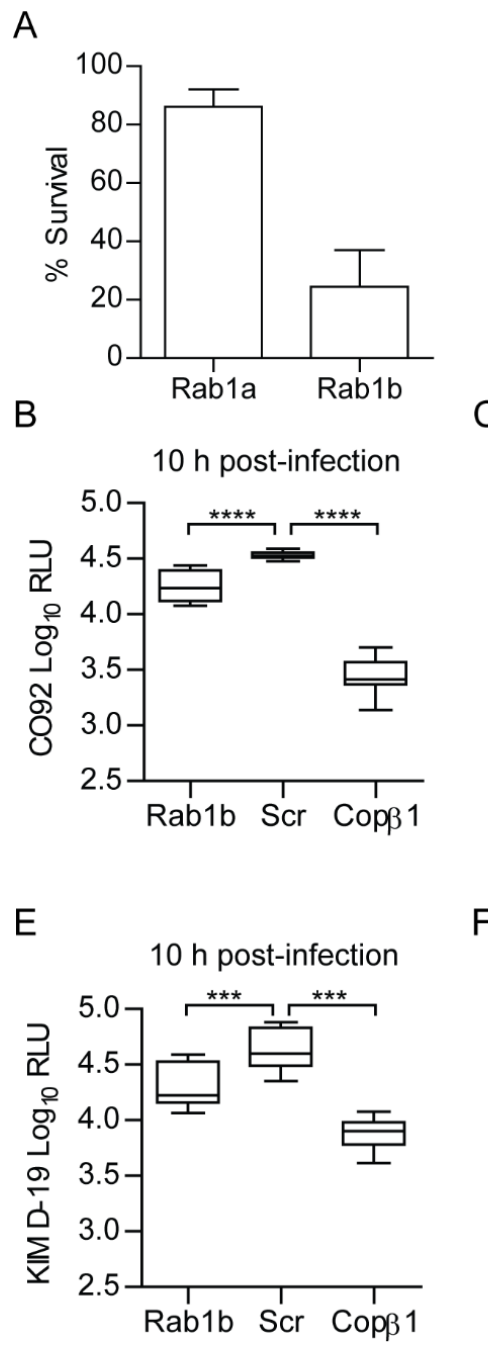

C

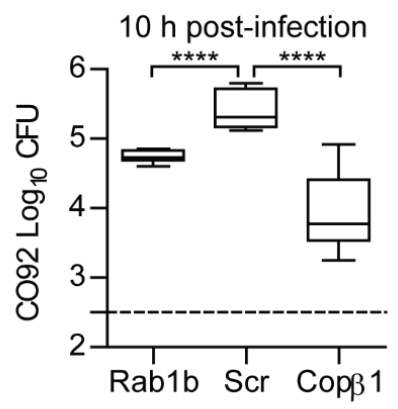

$\mathrm{F}$

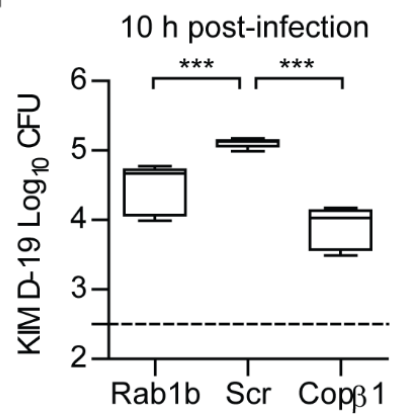

D

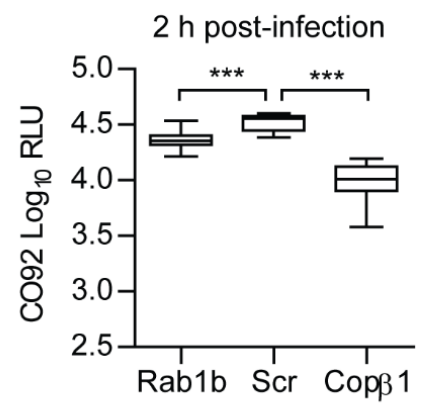

G

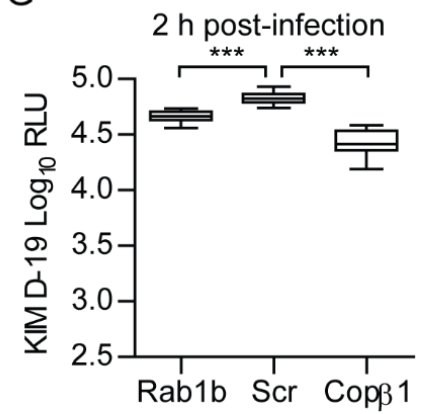

Figure 3-1. Rab1b knockdown inhibits the survival of $Y$. pestis within macrophages.

RAW264.7 macrophages were reverse transfected with Rab1a, Rab1b, scrambled (Scr), or Cop $\beta 1$ siRNA. $48 \mathrm{~h}$ after transfection cells were infected with $Y$. pestis (MOI 10). (A) Percent survival of intracellular CO92 pCD1 ${ }^{(-)}$LuXPtolc in Rab1a or Rab1b siRNA treated macrophages as compared to Scr siRNA treated macrophages. (B) Bioluminescence of intracellular bacteria from macrophages infected for $10 \mathrm{~h}$ with Y. pestis CO92 pCD1(-) LuXPtolC. (C) Conventional enumeration of intracellular bacteria from macrophages infected for $10 \mathrm{~h}$ with Y. pestis CO92 pCD1(-) LuXPtolc. 
(D) Bioluminescence of intracellular bacteria from macrophages infected for $2 \mathrm{~h}$ with Y. pestis CO92 pCD1 ${ }^{(-)}$LuXPtolc. (E) Bioluminescence of intracellular bacteria from macrophages infected for $10 \mathrm{~h}$ with Y. pestis KIM D-19 LuXPtolc. (F) Conventional enumeration of intracellular bacteria from macrophages infected for $10 \mathrm{~h}$ with Y. pestis KIM D-19 LuXPtolc. (G) Bioluminescence of intracellular bacteria from macrophages infected for $2 \mathrm{~h}$ with Y. pestis KIM D-19 LuXPtolc. The limit of detection for conventional enumeration is denoted by the dotted line. RLU = Relative Light Units; CFU = Colony Forming Units. ${ }^{* * *}=p<0.001,{ }^{* * *}=p<0.0001$. 
A

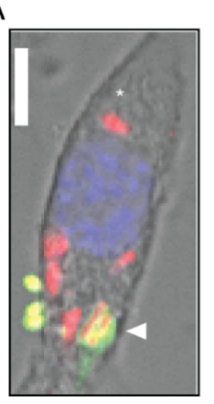

B

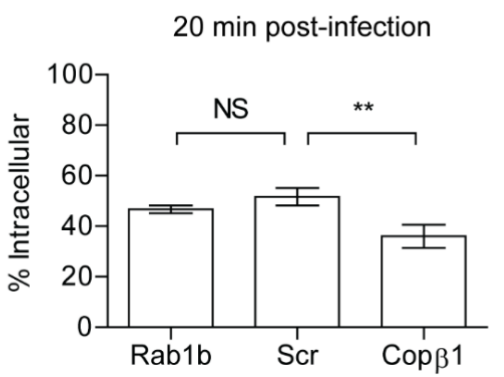

C

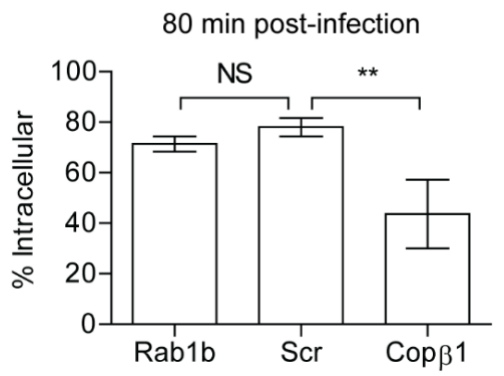

Figure 3-2. Rab1b knockdown does not impact Y. pestis invasion of macrophages.

RAW264.7 macrophages were reverse transfected with Rab1b, scrambled (Scr), or Copß1 siRNA. $48 \mathrm{~h}$ after transfection cells were infected with $Y$. pestis CO92 pCD1(-)pGEN- $P_{E M 7:: D s R E D(M O I}$ 7.5). 20 or 80 min post-infection cells and bacteria were fixed with paraformaldehyde and extracellular bacteria were stained by indirect immunofluorescence with anti- $Y$. pestis antibody. (A) Representative image showing differential staining of intracellular (red) and extracellular (green or yellow) bacteria. Scale bar is $5 \mu \mathrm{m}$. Asterisk denotes intracellular $Y$. pestis. (B and C) Percentage of intracellular bacteria calculated at 20 and 80 min post-infection, respectively. ${ }^{* \star}=p<0.01, n s=$ not significant. 
A
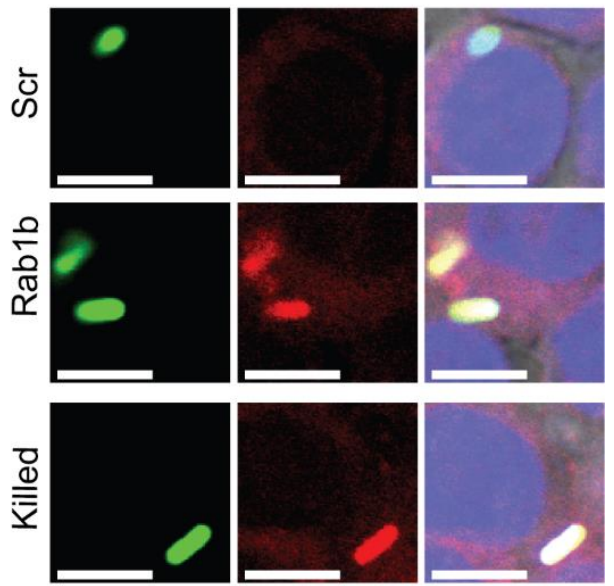

Y. pestis
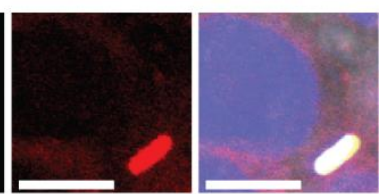

LysoT

Merge +

DAPI

B

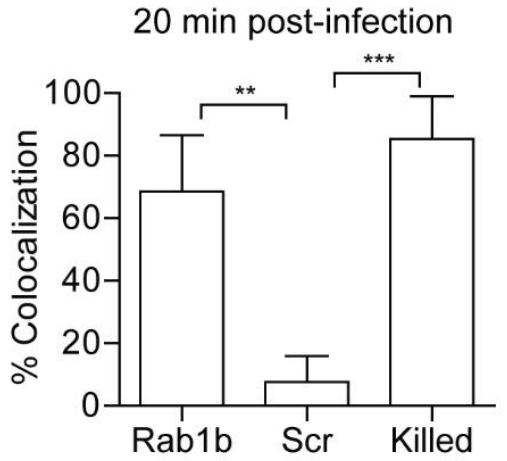

C

80 min post-infection

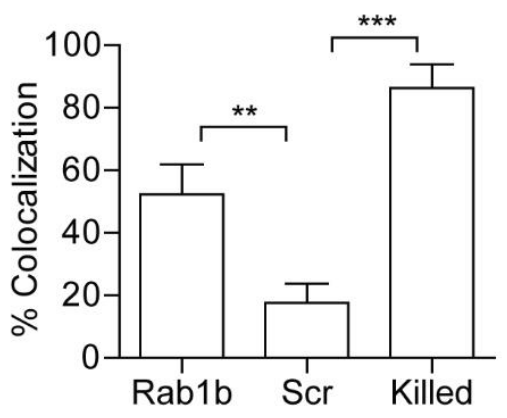

Figure 3-3. Rab1b knockdown alters YCV acidification.

RAW264.7 macrophage cells were reverse transfected with scrambled (Scr), Rab1b or Cop $\beta 1$ siRNA. $48 \mathrm{~h}$ after transfection cells were incubated with Lysotracker Red DND-99 for $1 \mathrm{~h}$ and then infected with live or paraformaldehyde-killed $Y$. pestis CO92 pCD1(-) pGEN222 expressing EGFP (MOI 7.5). Colocalization of Lysotracker Red DND-99 and Y. pestis CO92 pCD1(-) pGEN222 was 
determined by confocal microscopy. (A) Representative images showing colocalization of Lysotracker Red DND-99 and Y. pestis. Scale bar is $5 \mu$ m. (B) Percent of YCVs that colocalized with Lysotracker Red DND-99 at 20 min post-infection. (C) Percent of YCVs that colocalized with Lysotracker Red DND-99 at 80 min post-infection. ${ }^{* *}=p<0.01,{ }^{* * *}=p<0.001$. 
A

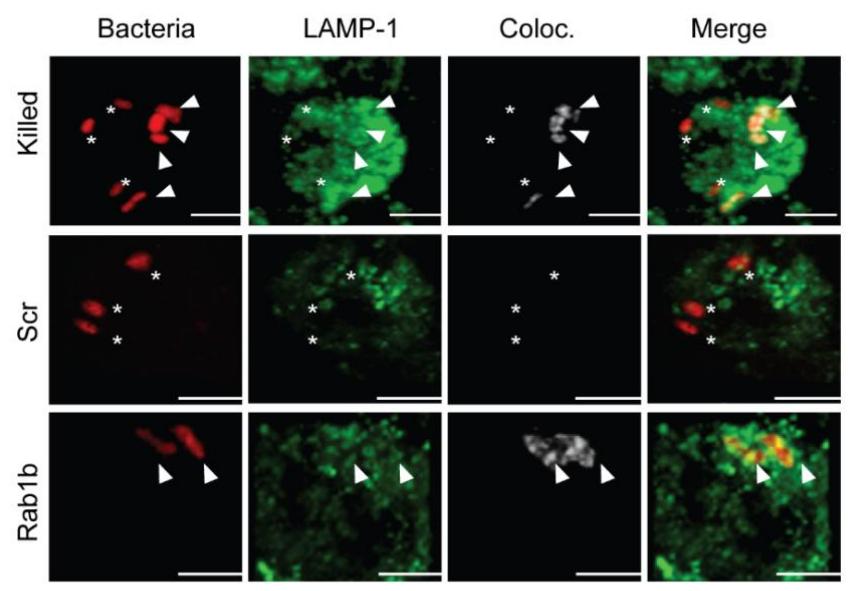

B

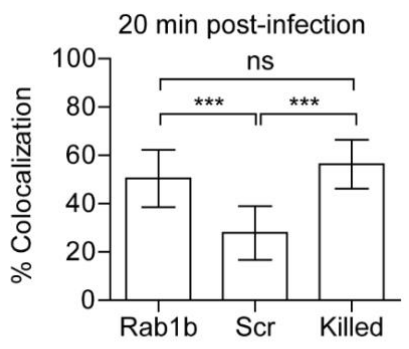

C

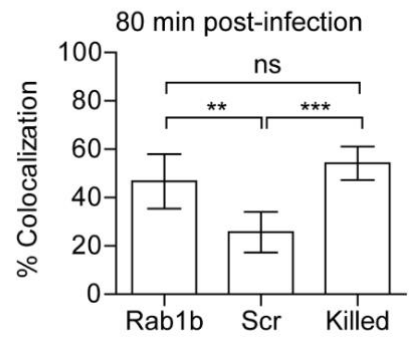

Figure 3-4. Rab1b knockdown increases YCV association with Lamp1.

RAW264.7 macrophage cells were reverse transfected with either scrambled (Scr) or Rab1b siRNA. $48 \mathrm{~h}$ after transfection cells were infected with live or paraformaldehyde-killed $Y$. pestis CO92 pCD1(-)pGEN-EM7::DsRED(MOI 3). Cells were stained for Lamp1 and colocalization was determined by confocal microscopy. (A) Representative images showing bacterial colocalization with Lamp1 at 20 min post-infection. Colocalization channel was defined using Imaris software. Asterisks denote bacteria not colocalized with Lamp1; arrowheads denote bacteria colocalized with Lamp1. Scale bar is $5 \mu \mathrm{m}$. (B) Percent of YCVs that colocalized with Lamp1 at 20 min post-infection. (C) Percent of YCVs that colocalized with Lamp1 at 80 min post-infection. ${ }^{* *}=p<0.01,{ }^{* *}=p<0.001$. 


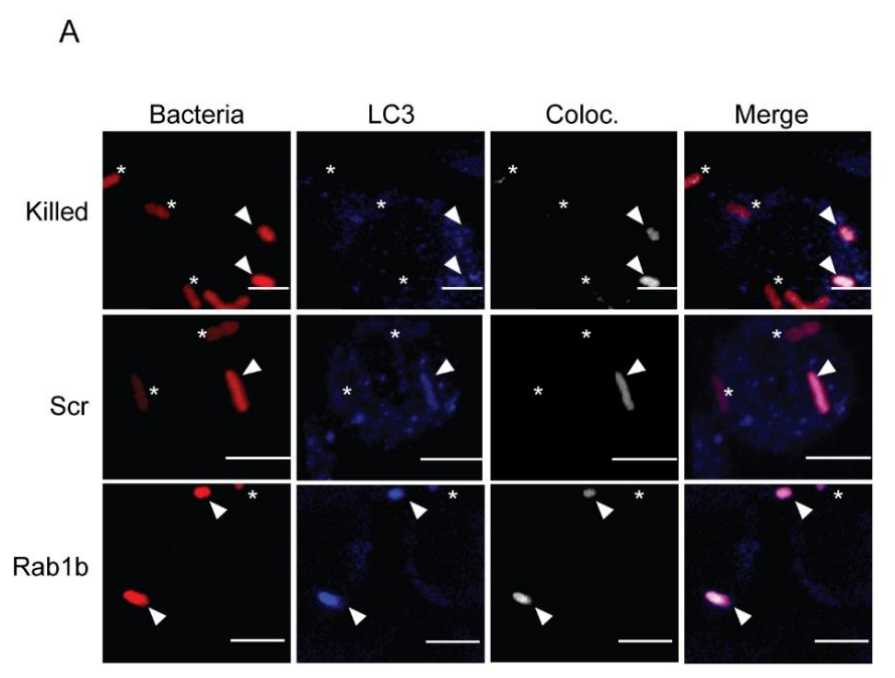

B

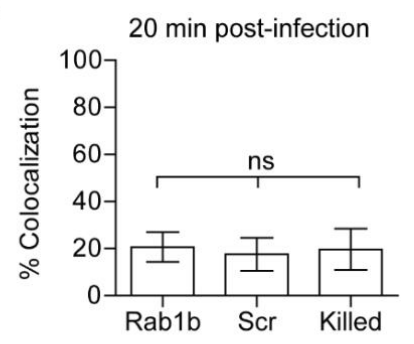

C

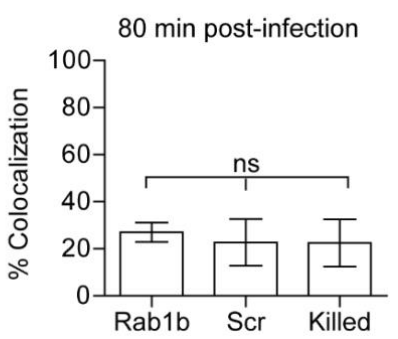

Figure 3-5. Rab1b knockdown does not affect YCV association with LC3.

RAW264.7 macrophage cells were reverse transfected with either scrambled (Scr) or Rab1b siRNA. $48 \mathrm{~h}$ after transfection cells were infected with live or paraformaldehyde killed $Y$. pestis

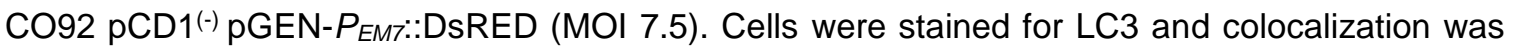
determined by confocal microscopy. (A) Representative images showing bacterial colocalization with LC3 at 20 min post infection. The colocalization channel was defined using Imaris software. Asterisks denote bacteria not colocalized with LC3; arrowheads denote bacteria colocalized with LC3. Scale bar is $5 \mu \mathrm{m}$. (B) Percent of YCVs that colocalized with LC3 at 20 min post-infection. (C) Percent of YCVs that colocalized with LC3 at 80 min post-infection. ns $=$ not significant. 


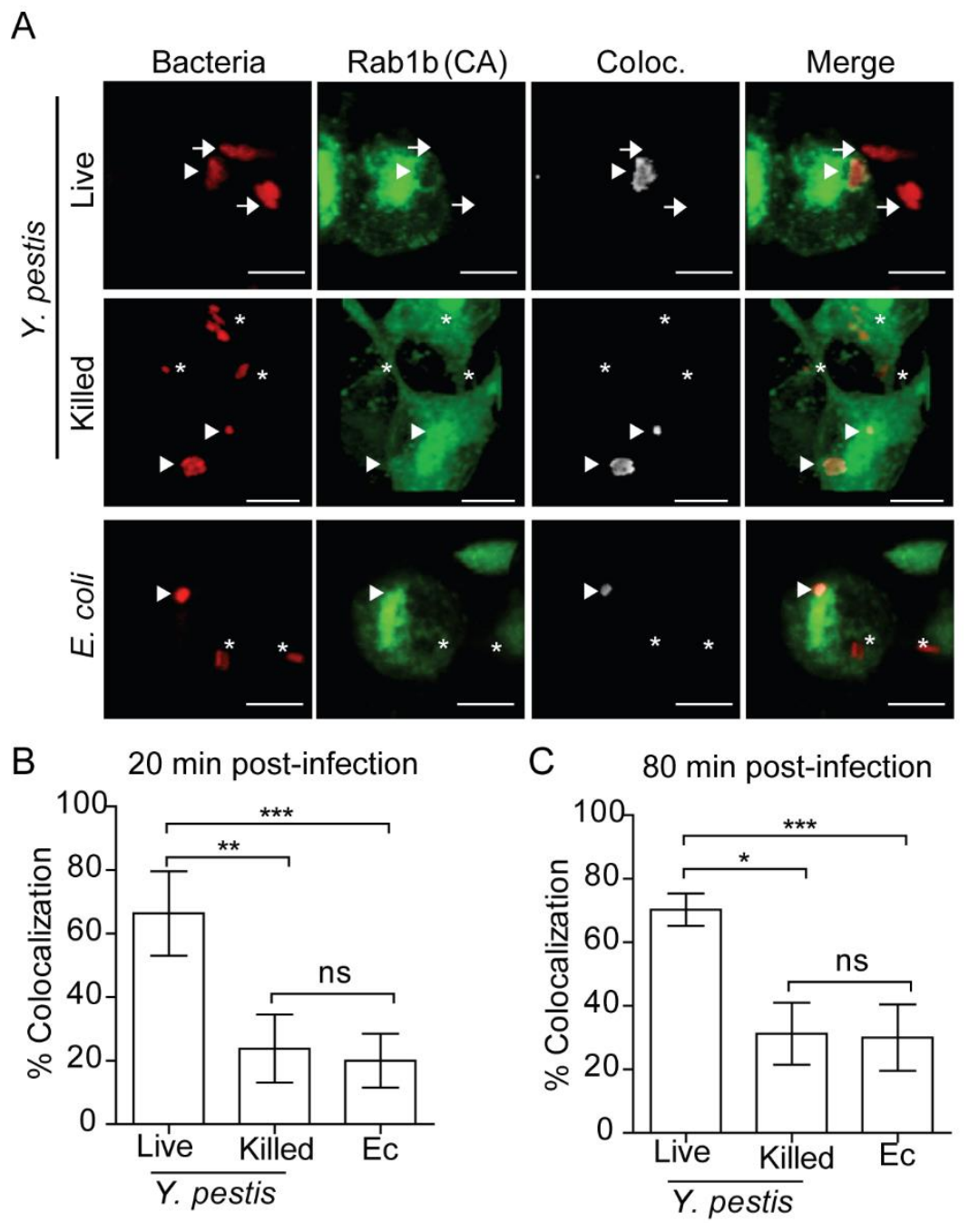

Figure 3-6. Rab1b is recruited to the YCV

RAW264.7 macrophages were transiently transfected with pEGFP-Rab1B(CA). $24 \mathrm{~h}$ after transfection cells were infected with either live or paraformaldehyde killed Y. pestis pMCherry (MOI 7.5) or E. coli pMCherry (MOI 20). Colocalization of EGFP-Rab1b(CA) and bacteria was determined by confocal microscopy. (A) Representative images showing bacterial colocalization with EGFP-Rab1b(CA). Colocalization channel was defined using Imaris software. Asterisks denote bacteria not colocalized with EGFP-Rab1b(CA); arrowheads denote bacteria colocalized with EGFP-Rab1b(CA); arrows denote bacteria in untransfected cells. Scale bar is $5 \mu \mathrm{m}$. (B and C) Percent of bacteria colocalized with EGFP-Rab1B(CA) at 20 and 80 min post-infection. ${ }^{*}=p<0.05$; ${ }^{* *}=p<0.01 ;{ }^{* * *}=p<0.001$ 
A

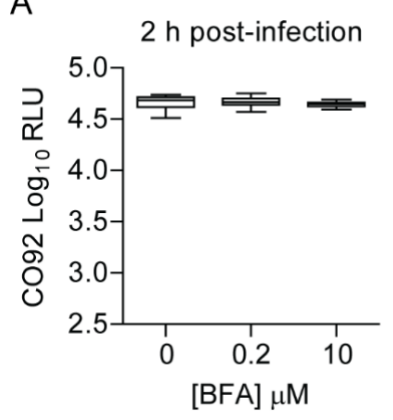

C

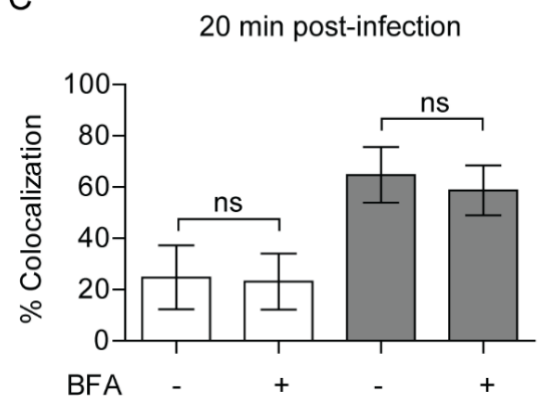

B

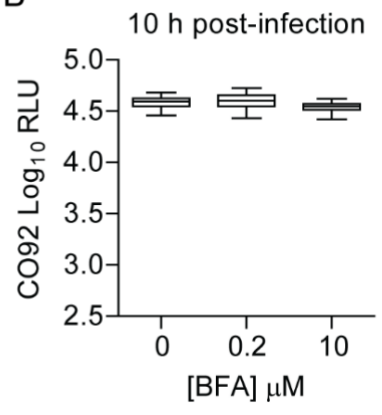

$\mathrm{D}$

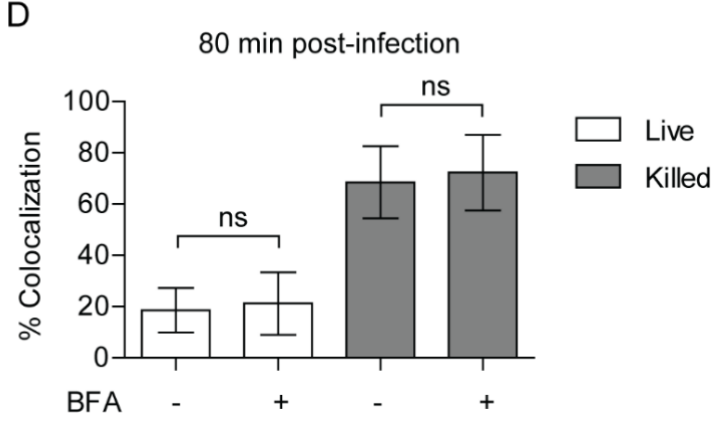

Figure 3-7. Inhibition of the secretory pathway does not inhibit $Y$. pestis intracellular survival.

RAW264.7 macrophages were treated with $0,0.2$ or $10 \mu \mathrm{M}$ BFA prior to infection with $Y$. pestis CO92 pCD1(-) LuXPtolc (MOI 10). Extracellular bacteria were killed with gentamicin and intracellular bacterial numbers were monitored at (A) $2 \mathrm{~h}$ and (B) $10 \mathrm{~h}$ post-infection by bioluminescence. To determine if BFA treatment impacted the ability of $Y$. pestis to inhibit YCV acidification, macrophages treated with $10 \mu \mathrm{M}$ BFA were incubated with Lysotracker Red DND-99 prior to infection with live or paraformaldehyde killed Y. pestis CO92 pCD1(-) pGEN222 (MOI 3). Bacterial Colocalization with Lysotracker Red DND-99 was determined by confocal microscopy at (C) 20 and (D) 80 min post-infection. $\mathrm{ns}=$ not significant. 
A

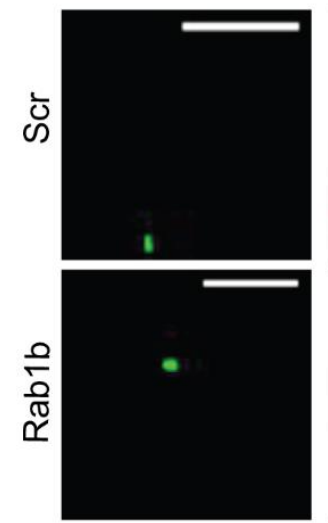

L. pneumophila
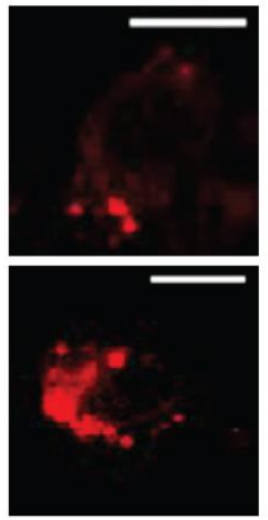

LysoT
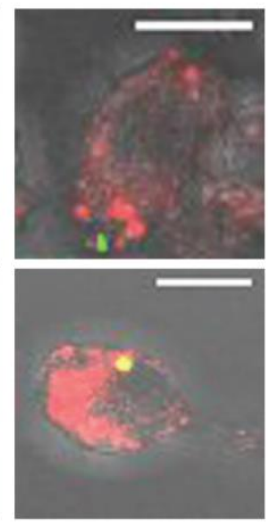

Merge

B 20 min post-infection

C 80 min post-infection
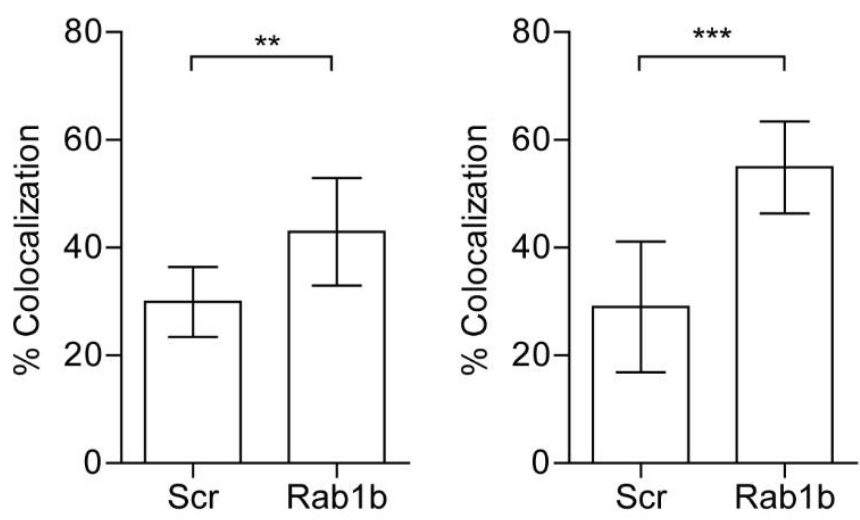

\section{Figure 3-8. Knockdown of Rab1b Increases L. pneumophila LCV acidification.}

RAW264.7 macrophage cells were reverse transfected with either scrambled (Scr) or Rab1b siRNA. $48 \mathrm{~h}$ after transfection cells were incubated with Lysotracker Red DND-99 for $1 \mathrm{~h}$, and infected with L. pneumophila pMIP-GFP (MOI 10). Coverslips were fixed and colocalization of Lysotracker was determined by confocal microscopy. (A) Representative images showing colocalization of Lysotracker with L. pneumophila. Scale bar is $5 \mu \mathrm{m}$. (B) Percent of LCVs that colocalized with Lysotracker at 20 min post-infection. (C) Percent of LCVs that colocalized with Lysotracker Red DND-99 at 80 min post-infection. ${ }^{* *}=p<0.05,{ }^{* * *}=p<0.001$. 

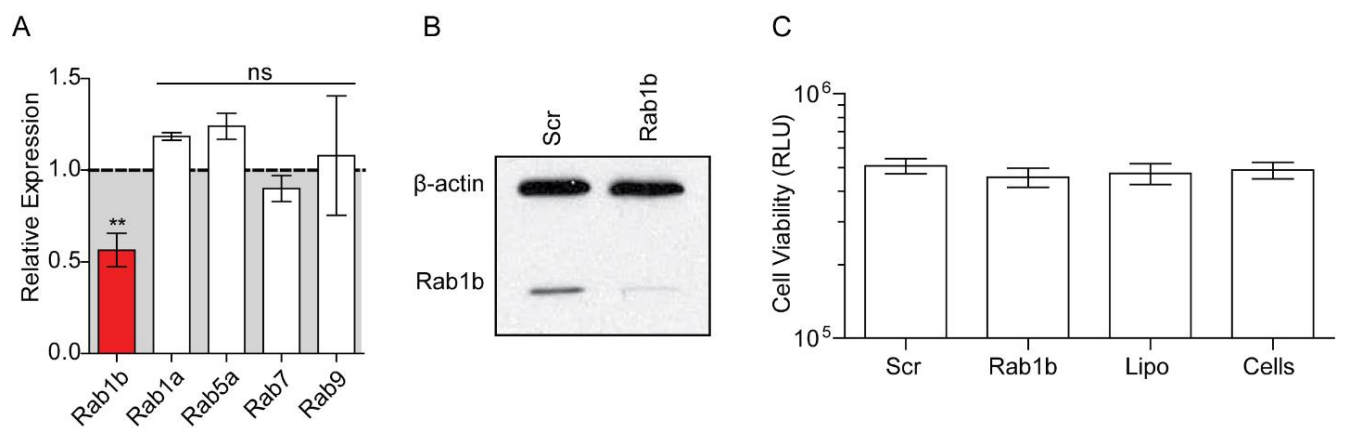

\section{Figure 3-9. S1 Transfection with Rab1b siRNA in macrophages}

RAW264.7 macrophages were reverse transfected with either scrambled (Scr) or Rab1b siRNA and incubated for 48 h. (A) Total RNA was isolated from transfected cells $(n=5)$ and Rab1b, Rab1a, Rab5a, Rab7 and Rab9 transcript levels were determined by qRT-PCR (Rab1b primers: 5'TGTCCTTTGTGCTGTCTCTTG -3' and 5'- TCATCCTTTTCCATCTTCCCC -3'; Rab1a primers: 5'CCTGCCTTCTCCTTAGGTTTG -3' and 5'- TCGAAATCTTTCCTGGCCTG -3'; Rab5a primers: 5'TGGTCAAGAACGGTATCATAGC -3' and 5'- GCCTTTGAAGTTCTTTAACCCAG -3'; Rab7 primers: 5'- AATAGGAGCGGACTTTCTGAC -3' and 5'- CATCAAACACCAGAACACAGC -3'); Rab9 primers: 5'- CACGGAAGATAGGTCAGAACAC -3' and 5'- CCCTTTAATGCCATCAACAGC -3'); GapDH primers: 5'- AATGGTGAAGGTCGGTGTG -3' and 5'- ACAAGCTTCCCATTCTCGG 3'). Relative expression was calculated using the $\Delta \Delta$ Ct method [228]. Only Rab1b levels were significantly altered in Rab1b siRNA-treated cells compared to scramble treated cells ${ }^{* *}=p<0.01$; Student's T-test). (B) Whole cell lysates were harvested from transfected cells and Rab1b protein levels (anti-Rab1b(G-20); Santa Cruz sc-599) were determined by Western blot. $\beta$-actin (anti- $\beta$ Actin; Abcam ab8227) represents loading control. (C) Cell viability of Rab1b siRNA transfected cells was determined using Cell Titer-Glo as described by the manufacturer (Promega). No significant difference in viability was observed between Rab1b siRNA treated cells and scramble siRNA-treated (Scr), untransfected macrophages (Cells), or macrophages treated with Lipofectamine without siRNA (Lipo). RLU = Relative Light Units. 
A

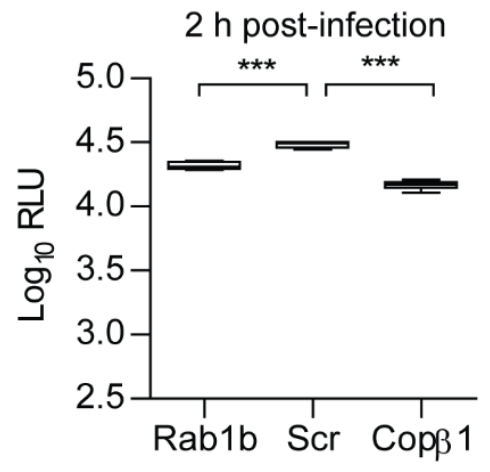

B

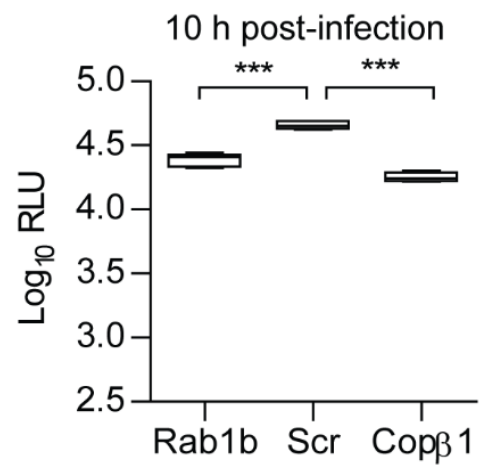

Figure 3-10. S2 Growth at $37^{\circ} \mathrm{C}$ does not alter intracellular survival of $Y$. pestis.

RAW264.7 macrophages were reverse transfected with Rab1b, scrambled (Scr), or Cop $\beta 1$ siRNA. $48 \mathrm{~h}$ after transfection cells were infected with Y. pestis CO92 pCD1(-) LuXPtolC $(\mathrm{MOI} 10)$ grown for $3 \mathrm{~h}$ at $37^{\circ} \mathrm{C}$ prior to infection. (A) Bioluminescence of intracellular bacteria from macrophages infected for $2 \mathrm{~h}$. (B) Bioluminescence of intracellular bacteria from macrophages infected for $10 \mathrm{~h}$. $R L U=$ relative light units. ${ }^{* * *}=p<0.001$ 
A

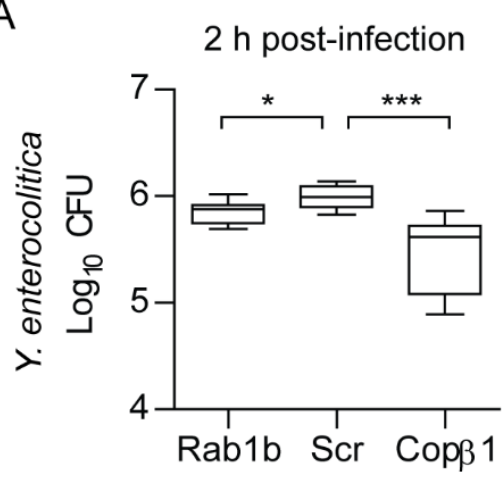

C

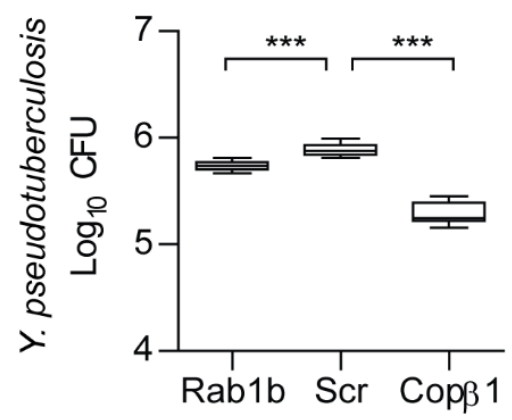

B

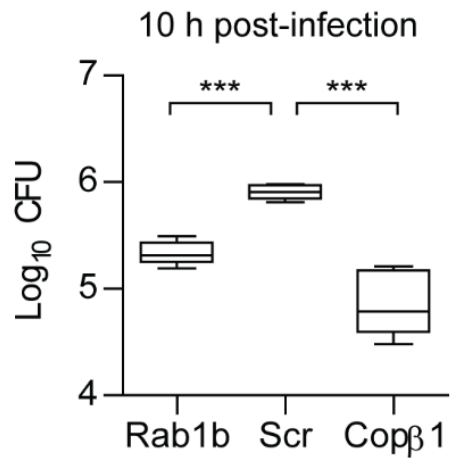

D

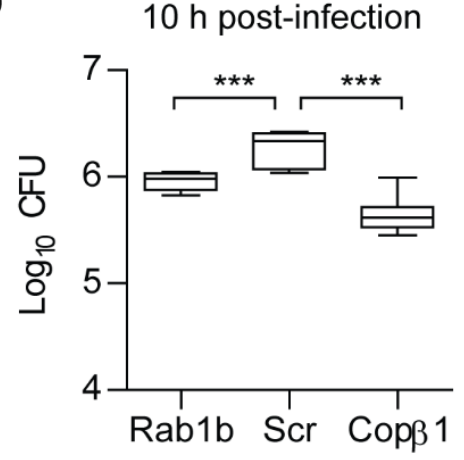

Figure 3-11. S3 Rab1b knockdown inhibits the survival of enteric Yersinia within macrophages.

RAW264.7 macrophages were reverse transfected with Rab1b, scrambled (Scr), or Cop $\beta 1$ siRNA. $48 \mathrm{~h}$ after transfection cells were infected with pYV cured $Y$. enterocolitica 8081 [229] or $Y$. pseudotuberculosis IP32952 [17](MOI 10). Extracellular bacteria were killed with gentamicin and at 2 and $10 \mathrm{~h}$ post infection intracellular bacteria were determined by conventional enumeration. (A) Intracellular Y. enterocolitica at $2 \mathrm{~h}$ post infection. (B) Intracellular $Y$. enterocolitica at $10 \mathrm{~h}$ post infection. (C) Intracellular Y. pseudotuberculosis at $2 \mathrm{~h}$ post-infection (D) Intracellular $Y$. pseudotuberculosis at $10 \mathrm{~h}$ post-infection. The limit of detection for conventional enumeration is $2.5 \log _{10}$ CFU. CFU $=$ colony forming units. ${ }^{*}=p<0.05,{ }^{* * *}=p<0.001$ 
$\underline{\text { Tables }}$

Table 3--1. Table S1 Bacterial strains used in these studies.

Table S1. Bacterial Strains

Bacterial Strains

Y. pestis CO92 pCD1(-)

References/sources

Y. pestis KIM D-19 pgm(') pCD1 $^{(+)}$

[9]

Y. pseudotuberculosis IP32593 pYV(-)

BEI Resources

Y. enterocolitica 8081 pYV(-)

E. coli DH5a

L. pneumophila AA100

[17]

[229]

New England Biolabs

[224] 
CHAPTER 4:

GENOME-WIDE RNAI HIGH-THROUGHPUT SCREEN IDENTIFIES THAT YERSINIA PESTIS EXPLOITS THE HOST ENDOCYTIC RECYCLING PATHWAY FOR INTRACELLULAR SURVIVAL 
$\underline{\text { Introduction }}$

Yersinia pestis is a facultative intracellular pathogen that causes the human disease known as plague $[1,5]$. There have been three major plague pandemics, the most noted being the Black Death during the $14^{\text {th }}$ century [1]. Recent epidemiological data demonstrates that $Y$. pestis is endemic in many countries, including the United States, and have led to the World Health Organization classifying plague as a re-emerging disease [1, 5]. In 2015, the Centers for Disease Control and Prevention (CDC) reported 15 human plague cases in the United States with three fatalities [6]. There are three forms of human plague: bubonic, pneumonic, and septicemic plague. Each form of plague results in an acute infection but is highlighted by the tissues primarily colonized by $Y$. pestis. Bubonic plague is the most common form of human plague and arises after a bite from a $Y$. pestis infected flea. The bacteria rapidly disseminate from the inoculation site through the lymphatic system and colonize the draining lymph node [1, 49, 184]. Eventually the bacteria enter and replicate in the bloodstream, leading to the formation of septicemic plague $[1,6]$. In rare cases, $Y$. pestis can be directly inoculated into the blood by a flea or from the bite of an infected animals, resulting in primary septicemic plague without colonization of the lymphatic system [1]. From the blood, Y. pestis is distributed throughout the body and colonizes other tissues such as the spleen, liver and lungs. Colonization of the lungs leads to the development of secondary pneumonic plague and the potential for patients to aerosolize $Y$. pestis by coughing and person-to-person transmission. Inhalation of infected aerosols by naïve individuals can result in colonization of the lungs by $Y$. pestis and the development of primary pneumonic plague. All three forms of plague are very rapid infections with high mortality rates in the absence of early antibiotic treatment. $[1,230]$. Furthermore, the ability for aerosol transmission of the $Y$. pestis raises the potential for this bacterium to be used a biological weapon [14].

In the environment, $Y$. pestis is maintained through a zoonotic transmission cycle between rodents and fleas $[1,5]$. The ability of $Y$. pestis in exist in these two very different hosts is a result of the acquisition virulence factors required for the mammalian host and transmission factors required for flea colonization $[1,42,48,231]$. The bacterium regulates these two groups of factors accordingly to ensure expression of appropriate factors only when required $[1,41,42,48,231$, 
232]. For example, important antiphagocytic factors expressed during mammalian infection, like the Ysc type three secretion system (T3SS), secreted Yop effectors, and the Caf1 capsule, are not required for flea infection and are thus repressed in the flea vector[1]. However, the repression of these antiphagocytic factors in the flea means that during the initial colonization of the mammalian host during bubonic plague, $Y$. pestis is not expressing important virulence factors. Therefore, there is a transition period when the bacteria are highly susceptible to recognition and phagocytosis by macrophages and neutrophils immediately upon flea transmission [51, 54]. This susceptibility is highlighted by intravital microscopy of the infection site by Shannon et al. [233]. Following flea transmission of $Y$. pestis to the dermis of the ear, polymorphonuculear leukocytes (PMNs) are rapidly recruited to the infection site and appear to phagocytosis the bacteria. To a lesser degree, host macrophages are also recruited and engulf bacteria. Interestingly, infected macrophages appeared to migrate away from the infection site. Importantly, growing evidence suggests that these two phagocytes have very different abilities to kill $Y$. pestis [51, 53, 58, 80-82, 233]. Specifically, neutrophils appear to be much more efficient at killing phagocytosed bacteria than macrophages $[53,58,80]$. Moreover, several studies suggest that $Y$. pestis actively inhibits killing by both mouse and human macrophages [54, 55, 57, 59, 82, 83, 86, 152].

Intracellular $Y$. pestis have been isolated from macrophages in both rodent and nonhuman primate models of plague and from plague infected patients $[49,58,84,234,235]$. These findings suggest $Y$. pestis intracellular survival may contribute to virulence. Supporting this hypothesis, Ye at el. showed that animals selectively depleted of macrophage/dendritic cell populations exhibited delayed dissemination and subsequently lower bacterial burdens during plague infection [52]. Moreover, the $Y$. pestis phoPQ mutant, which is defective for survival within macrophages $[56,83$, $86,90]$, has a 75 -fold attenuation in subcutaneous infection of BALB/c mice and a significant delay in the development of lethal disease in Swiss Webster mice [56, 90]. In contrast, studies with canine macrophages, a species relatively resistant to plague [62], demonstrated that $Y$. pestis is defective in intracellular survival in these cells compared to murine macrophages [62, 91]. These data, combined with studies showing the sensitivity of $Y$. pestis to PMN phagocytic killing $[53,58$, 
80], suggest that $Y$. pestis infection of macrophages may provide an intracellular niche to avoid killing by PMNs during early stages on infection.

Upon phagocytosis by macrophages, Y. pestis quickly and actively inhibits the normal maturation process of the phagosome $[54,55,57,59,83,86,152]$. A hallmark of this process is the inhibition of phagosome acidification by $Y$. pestis $[55,59]$. The bacterium remains within this phagosome throughout the course of the intracellular infection, eventually remodeling it into a compartment called the Yersinia containing vacuole (YCV). In addition to maintaining a neutral pH, a subset of the YCVs eventually mature into autophagosome-like compartments, acquiring both LC3-II and double membranes [88, 89]. During late infection, the YCV begins to expand from a tight fitting vacuole to a spacious vacuole, which coincides with bacterial replication $[55,59,62,86$, 88, 89]. While autophagy has been noted during $Y$. pestis infection, the extent to which it impacts intracellular survival is not fully understood [59]. However, studies with Y. pseudotuberculosis indicate autophagy is important for intracellular bacterial metabolism [88, 89].

Recently we identified the first host factor required by $Y$. pestis for the YCV biogenesis process [61]. We showed that the Rab GTPase Rab1b, which normally mediates ER-Golgi trafficking $[209,210]$, is rapidly recruited to the YCV and is required for $Y$. pestis to inhibit vacuole acidification and phagosome maturation within macrophages. While Rab1 has been shown to be recruited to the vacuole of several intracellular pathogens $[61,118,188,189,191,192,194]$, these studies were the first to link recruitment to avoidance of the phagosome acidification maturation. Furthermore, inhibition of pathogen-containing vacuole (PCV) acidification by Rab1 recruitment may be a conserved virulence mechanism for intracellular pathogens as RNAi of Rab1 during Legionella pneumophila infection also resulted in LCV acidification [61]. These data demonstrate that $Y$. pestis actively targets host factors to subvert macrophage phagosomal maturation and/or to generate a protective replicative niche within the macrophage. Here we attempt to identify additional host factors required for $Y$. pestis intracellular survival by developing an RNAi-based, high throughput assay to monitor the impact of host factors on $Y$. pestis survival in macrophages. Using this assay, we performed a whole genome screen and identified 135 genes contributing to Y. pestis intracellular survival. Network analysis of these genes revealed enrichment for factors 
involved in host cell recycling pathway. Based on these findings, we demonstrate that $Y$. pestis actively remodels the YCV to resemble host recycling endosomes. Finally, we demonstrate that $Y$. pestis infection also disrupts normal host cell recycling, likely through sequestration of Rab11.

$\underline{\text { Results }}$

Development of a high throughput assay to monitor the impact of host gene RNAi on Y. pestis intracellular survival

RNAi has been used to identify host factors required for intracellular survival of several pathogens [130-133, 135-142]. However, most of these screens have used Drosophila or HeLa cell lines, which may not present the same hurdles to intracellular survival as a pathogen may encounter during infection of macrophages. As macrophages are specifically infected during $Y$. pestis infection, our first goal was to select a macrophage cell line that was amendable to Lipofectamine-mediated transfection/RNAi needed for high-throughput screening. Towards this end, we tested siRNA transfection and knockdown in several human and mouse macrophage cell lines. While robust RNAi was observed in mouse macrophages, we were unable to reproducibly knockdown gene expression in human cell lines (data not shown). Based on these results, we chose RAW264.7 mouse macrophages for further optimization. Using a combination of siRNAs targeting genes of variable expression levels, we optimized Lipofectamine/siRNA concentrations and the transfection time to consistently achieve $>70 \%$ knockdown of targets (Fig. 4-1A). We also confirmed the transcript knockdown correlated with decreased protein levels for GAPDH and COPB1 (Fig. 4-1B). Next, we established whether we could monitor changes $Y$. pestis intracellular survival in siRNA-treated macrophages. Macrophages were transfected with siRNAs targeting Rab2A and Cop $\beta 1$, genes shown to impact the intracellular survival of multiple pathogens [130, 135, 138, 140, 141] and infected with $Y$. pestis CO92 pCD1(-)LuXPtolC [151]. This bioluminescent bioreporter can differentiate as little as a 2-fold difference in macrophage intracellular burdens (Fig. 4-1C) and bioluminescence directly correlates to intracellular bacterial numbers (Fig. 4-1D; $R^{2}=$ 0.89). Transfection with these two siRNAs significantly inhibited $Y$. pestis intracellular survival compared scrambled siRNA treated macrophages (Fig. 4-1E), demonstrating the utility of this 
approach to identify host factors required for $Y$. pestis intracellular survival. Finally, using Cop $\beta 1$ siRNA as a positive control, we determined the robustness of our assay by establishing a Z' factor. $Z^{\prime}$ factors between 0.5 and 1 represent highly reproducible assays that are amenable to high throughput analysis [143, 144, 149]. At 2 and 10 hours post infection, we calculated a $Z^{\prime}$ factor of 0.61 and 0.83 , respectively, indicating a highly robust assay (Fig. 4-1F). Together these results indicate a robust assay that can be used for high throughput screening of host factors required for Y. pestis intracellular survival (Fig. 4-1G).

\section{Genome-wide siRNA screen identifies host cell signal transduction, transport and localization}

\section{pathways are required for $Y$. pestis intracellular survival.}

To identify host factors required for Y. pestis intracellular survival, RAW264.7 macrophages were reverse transfected with siRNAs targeting 17,370 genes, representing the whole mouse genome, and infected with Y. pestis CO92 pCD1(-)LuXPtolc. Each plate also contained control wells transfected with scrambled or COP $\beta 1$ siRNAs. Bioluminescence was measured at 2 and $10 \mathrm{~h}$ postinfection and $Z^{\prime}$ factors were calculated from the control wells (the average $Z^{\prime}$ factor for the screen at 2 and $10 \mathrm{~h}$ were 0.57 and 0.66 , respectively). Plates with $Z^{\prime \prime}$ factor less than 0.3 were repeated. At $12 \mathrm{~h}$ post-infection cell viability was also determined for 14,203 of the genes. Bioluminescence was normalized for each plate based on control wells and changes in $Y$. pestis intracellular survival were ranked by normalized scores (Fig 4-2A). 302 siRNAs that inhibited bacterial growth and 39 siRNAs that promoted bacterial growth were selected for secondary validation based on selection criteria outlined in the Materials and Methods. 23 additional siRNAs associated with autophagy were also chosen for further validation. For secondary validation, RAW264.7 macrophages were transfected with siRNAs targeting the 364 primary screen hits and subsequently infected with two

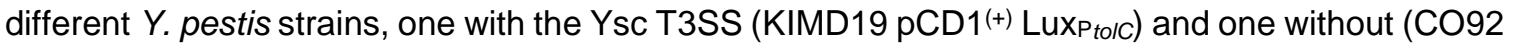
pCD1 ${ }^{(-)}$LuXPtolC). Bioluminescence was measured at 2 and $10 \mathrm{~h}$ post-infection and normalized scores from each strain were compared (Fig 4-2B). A direct correlation was observed between the two strains $\left(r_{s}=0.87\right)$ supporting previous studies that have shown that the T3SS is dispensable for intracellular survival $[54,61,83,151]$. From the primary hits, we confirmed that 135 siRNAs showed 
$\geq 40 \%$ inhibition of $Y$. pestis intracellular survival and 7 siRNAs showed a hypervirulent phenotype with $\geq 20 \%$ more growth than scrambled controls (Table 4-1).

To identify specific host pathways that may have been enriched for by our screen, we used a combination of Gene Ontology (GO) clustering and network analyses on our validated data set. ClueGO, a third party Cytoscape app, clusters genes under their GO term in a non-redundant manner, while preserving the highest enrichment of the parent GO term by applying a two sided hypergeometric test and kappa score to generate significance testing (for review see [236, 237]). Using all GO evidence codes, a minimum kappa score of 0.4 , and a p-value threshold of 0.05 , we observed substantial clustering within the validated dataset (Fig. 4-3A). Of the five enriched groups, the largest clustering enrichment was under Rab protein signal transduction ( $p V \leq 0.001$; Fig. 43B). Additional enrichment included vesicle-mediated transport, vacuole organization, vesicle docking and mitochondrion organization (Fig. 4-3B). Under the parent GO Term clusters, detailed GO terms significantly focused on host trafficking networks, transport and localization were common themes (Fig. 4-3C).

Based on the significant enrichment for Rab GTPase signal transduction in the GO ontologies, we next determined the degree to which the nine validated Rab GTPases (1b, 2b, 3d, $4 a, 19,20,23,30, \& 40 b)$ interacted within our validated screen hits. Using the GTPases as focal points, we generated a map of direct interactions from these Rab GTPases within our validated screen hits. These interactions were mapped using a combination of STRING and BioGRID databases to create a Circos plot with known interactions (gray), activators (green), and inhibitors (red) (Fig 4-4A). The Circos plot showed 8\% (11 genes) of the validated hits directly interact with these validated Rab GTPases. Next, we sought to identify the degree to which the validated Rab GTPases interacted within the validated and primary hit interactome. To do this, we identified direct interactions between all validated genes, and then expanded this analysis to include direct interactions with all primary screen hits using the STRING and BioGRID databases. These data were used to generate a map of the interactome using Cytoscape (Fig. 4-4B). This interactome showed that $59 \%$ of the genes identified in the primary screen and $28 \%$ of validated genes directly interacted with one another and that several of the Rab GTPases were part of this interactome. 
Together this data suggest that specific Rab-mediated host traffic pathways are targeted by $Y$. pestis in order to subvert phagosome maturation and intracellular survival. Host cell recycling is essential for $Y$. pestis survival

\section{Host cell recycling is essential for Y. pestis survival}

Rab GTPases are well studied regulators of cellular trafficking and endocytic pathways [185]. Our initial bioinformatic analysis of validated genes emphasized Rab-mediated endocytic trafficking as essential for $Y$. pestis intracellular survival. The Rab GTPases from our validated dataset could be categorized into three trafficking pathways: 1) host cell recycling (Rab4a, 20, 23, \& 30); 2) retrograde trafficking (Rab1b \& 2b); and 3) the secretory pathway (Rab40b) [99, 101, 102, 185, 238, 239]. As there appeared to be significant enrichment for Rab proteins involved in host cell recycling, we focused on this pathway for further analysis.

Rab4a, Rab11b and Myo5b are well characterized contributors to host cell recycling [238]. While Rab4a was a validated gene in our screen, the other two genes did not pass our primary screen criteria ( $\geq 60 \%$ inhibition of $Y$. pestis survival; $<50$ cytotoxicity) - Rab11b only inhibited $Y$. pestis survival by $50 \%$ in the primary screen and Myo5B was cytotoxic (upon subsequent analysis, only one of the three Myo5B siRNAs used in the primary screen was cytotoxic; data not shown). However, because of the importance of these proteins in the recycling pathway, we chose to independently verify the contribution of Rab4a, Rab11b and Myo5b on $Y$. pestis intracellular survival. Toward this end, RAW264.7 macrophages were transfected with single siRNAs targeting each of the three genes. Transfection with each siRNA resulted in $>60 \%$ knockdown on each gene target (Fig. 4-5A) with no significant loss in cell viability (Fig 4-5B). Subsequent infection with $Y$. pestis CO92 pCD1(-)LuXPtolC confirmed that knockdown of all three genes impacted the ability of $Y$. pestis to survive within the macrophage (Fig. 4-5C-F). Knockdown of Rab4a had the largest impact, inhibiting $Y$. pestis survival by $40 \%$ at $2 \mathrm{~h}$ (Fig. 4-5C; $\mathrm{p}<0.001$ ) and $>80 \%$ at $10 \mathrm{~h}$ (Fig. 4-5D; $\mathrm{p}<0.001$ ). Interestingly, knockdown of Rab11b and Myo5B had no significant impact on $Y$. pestis survival at $2 \mathrm{~h}$ (Fig. 4-5C), but attenuated Y. pestis by $>40 \%$ at $10 \mathrm{~h}$ (Fig. 4-5D; $\mathrm{p}<0.001$ ). Bioluminescence data was confirmed at $10 \mathrm{~h}$ by conventional bacterial enumeration (Fig. 4-5E). Importantly, knockdown of Rab4a or Rab11b did not alter the expression of Rab GTPases involved 
with phagolysosome maturation (Fig. 4-6A \& B). Together these data indicate that $Y$. pestis requires the host recycling pathway to avoid killing by macrophages.

\section{$\underline{\text { Y. pestis requires Rab4a of the recycling pathway to avoid YCV acidification }}$}

The decreased Y. pestis bioluminescence observed at $2 \mathrm{~h}$ for the Rab4a RNAi-treated cells could be attributed to either decreased bacterial uptake or early survival in the phagosome. To differentiate between these two possibilities, RAW264.7 macrophages transfected with siRNA targeting Rab4a or Rab11b were infected with Y. pestis-GFP. 20 min post-infection, extracellular bacteria were differential stained and quantified by confocal microscopy (Fig. 4-7A \& B). The ratio of extracellular/total bacteria was compared to scrambled and Copß1 siRNA treated macrophages. RNAi inhibition of Copß1 has been previously shown to prevent RAW264.7 macrophage uptake of Y. pestis [61]. Using Cop $\beta 1$ as a positive control, we show $>35 \%$ inhibition of $Y$. pestis invasion (Fig. 4-7B). The data shows there is no significant difference between Rab4a or Rab11b treated cells in comparison to the negative control (Fig. 4-7B). These together suggested that Rab4a inhibition of $Y$. pestis survival was due to events impacting early YCV biogenesis post-invasion.

Similar to Rab4a, we have previously shown that RNAi of Rab1b resulted in significant reduction in $Y$. pestis intracellular survival at $2 \mathrm{~h}$ post-infection, which directly correlated to an increase in the frequency of YCV acidification [61]. Therefore, we next determined the impact of Rab4a, Rab11b and Myo5b RNAi on YCV acidification. To monitor YCV acidification, transfected macrophages were pulsed with Lysotracker Red DND-99 prior to infection with Y. pestis CO92 pCD1(-) pGEN222::EGFP. Lysotracker Red DND-99 is a fluorescent probe which accumulates in acidified vacuoles $(<\mathrm{pH} 5.5)$. As a positive control, macrophages were also infected with paraformaldehyde killed Y. pestis CO92 pCD1(-) pGEN222::EGFP [59, 61]. As previously shown, Y. pestis actively avoided YCV acidification with $<20 \%$ of YCVs colocalized with Lysotracker Red DND-99 by 80 min post-infection (Fig. 4-7E) [61]. In contrast, YCV containing paraformaldehyde killed $Y$. pestis is acidified as $>80 \%$ of YCVs were acidified by 80 mins post infection (Fig. 4-7E). As indicated by our RLU data, Rab4a knockdown increased YCV acidification to $55 \%$ by 20 min post-infection, and $>70 \%$ by 80 min post-infection ( $p \vee \leq 0.001$; Fig. 4-7D \&E). Our data shows that Rab4a and Rab11b are not required for $Y$. pestis invasion. However, Rab4a is required $Y$. pestis 
to avoid early YCV acidification. In contrast, Rab11b and Myo5b impact YCV acidification later in the process of intracellular survival as inhibition of these host factors impact acidification to a larger degree at 80 mins post-infection.

Interestingly, while there was a statistically significant increase in YCV acidification with the loss of Rab11b at 20 mins post infection (10\%; Fig. 4-7D) this did not reflect the $2 \mathrm{~h} \mathrm{RLU}$ data (Fig. 5C). Moreover, by 80 mins post infection the loss of Rab11b caused a $>40 \%$ of YCVs to acidify (Fig. 4-7D) which parallels our RLU data at 10 hours post infection (Fig. 4-5D). Finally, the loss of Myo5b had no effect on YCV acidification at 20 mins post infection, but by 80 mins post infection increased YCV acidification by $>40 \%$ (Fig. 4-7D), which again reflected bioluminescence data at 10 hours post infection (Fig. 4-5D). In summary, our findings demonstrate that Rab4a, Rab11b, and Myo5b are required for efficient avoidance of $\mathrm{YCV}$ acidification within the first 80 mins of infection. Furthermore, these data suggest that $Y$. pestis is actively exploiting the recycling endocytic pathway for YCV biogenesis.

\section{$\underline{\text { The Yersinia containing vacuole acquires recycling endosome markers }}$}

Rab GTPases exert their function through coordinated and sequential direct interactions on the vesicle membrane [102, 185]. Recycling endosome maturation/trafficking is initiated by Rab4a for fast recycling back to the plasma membrane on the early endosome [238]. In contrast, Rab4a is exchanged for Rab11b for the maturation/trafficking of slow recycling vesicles that reside spatially close to the Golgi-ERGIC space [238]. Therefore we postulated that $Y$. pestis hijacks the host endocytic recycling pathway by recruiting Rab4a to the early YCV, and then exchanges Rab4a for Rab11b in order to remodel the YCV as a slow recycling endosome. To determine if Rab4a or Rab11b are recruited to the YCV, RAW264.7 macrophages were transfected with plasmids expressing Rab4a or Rab11b fused to EGFP [108] followed by infection with either Y. pestis CO92 pCD1(-) pGEN::mCherry, paraformaldehyde killed Y. pestis CO92 pCD1(-) pGEN::mCherry, or $E$. coli pGEN::mCherry. Association with Rab4a or Rab11b was determined by confocal microscopy (Fig. 4-8A). Rab4a localized to paraformaldehyde killed $Y$. pestis and E. coli at $50 \%$ and $45 \%$ respectively by 20 mins post infection (Fig. 4-8B). In contrast, live $75 \%$ of live $Y$. pestis bacteria colocalized with Rab4a at 20 mins post-infection (Fig. 4-8B). The localization of Rab4a, at 20 mins 
post infection to both paraformaldehyde killed $Y$. pestis and E. coli associate and could reflect the nature of Rab4a on the early sorting endosome [238].

However, by 80 mins post infection there is substantial loss of Rab4a to both paraformaldehyde killed Y. pestis and E. coli, 30\% and 20\% colocalization respectively. In contrast, live $Y$. pestis still retains $65 \%$ colocalization. Of note, is the significant drop in Rab4a colocalization with live $Y$. pestis by 80 mins post infection. In respect to Rab11b, by 20 mins post-infection $75 \%$ of live YCVs colocalized with Rab11b in contrast to $20 \%$ for paraformaldehyde killed $Y$. pestis and E. coli (Fig. 4-8C). The association of Rab11b with live Y. pestis significantly increases to $85 \%$ ( $p \leq 0.05$ ) by 80 mins post-infection, whereas paraformaldehyde killed $Y$. pestis and E. coli remained at $20 \%$ (Fig. 4-8C). These data taken together indicate that $Y$. pestis is actively recruits, and potential retains, Rab4a and Rab11b to the YCV during early stages of infection.

\section{Y. pestis infection stalls host recycling}

Our data has shown that Rab4a and Rab11b, and indirectly host cell recycling, are required for $Y$. pestis to actively subvert the maturation of the phagosome. Recruitment of these proteins to the YCV also indicates that $Y$. pestis remodels its phagosome to resemble a recycling endosome. Because of these links to host cell recycling, we next tested whether infection with $Y$. pestis has an impact on host cell recycling of the infected macrophage. Toward this end, we monitored transferrin receptor (TfR) recycling using differential antibody labelling as a measure of global host cell recycling. As a positive control for this assay, Rab4a, which is required for efficient recycling of TfR [240-242], was knockdown by RNAi and compared to scramble siRNA transfected macrophages. In scramble control samples, intracellular TfR peaked at 10 min post-antibody labelling of the TfR and recycling was observed at 20 mins, with a decrease in the intracellular receptor (Fig. 4-9C). In contrast, while intracellular TfR also peaked at 10 min post-antibody labelling in Rab4a siRNA treated cells, the steady state level of intracellular receptor remained significantly elevated as compared the scrambled control ( $\mathrm{pV} \leq 0.001$; Fig. 4-9C). Importantly, TfR intensity per cell remained significantly ( $p \vee \leq 0.001$ ) elevated in the Rab4a siRNA treated cells even at $24 \mathrm{~h}$, indicating continued inhibition of recycling. 
To determine the impact of $Y$. pestis infection on recycling, RAW264.7 macrophages were treated with anti-TfR antibody and then infected with $Y$. pestis CO92 pCD1(-)pGEN::dsRed, paraformaldehyde killed Y. pestis CO92 pCD1(-)pGEN::dsRed, or E. coli K12 pGEN::dsRed. At 2 and $24 \mathrm{~h}$ post-infection, cells were fixed and intracellular TfR intensity per cell per field was determined by microscopy (Fig.4-10A). Unlike paraformaldehyde killed bacteria, infection with live Y. pestis stalled host recycling as early as $2 \mathrm{~h}$ post-infection, resulting in significantly higher intracellular TfR intensity per cell per field as compared to uninfected cells, and continued to impact recycling as long as $24 \mathrm{~h}$ post-infection (Fig. 4-10). Furthermore, we observed a dose response, with greater MOls of $Y$. pestis resulting in increased intracellular TfR intensity per cell per field. Importantly, infection with 10-fold higher numbers of $E$. coli K12 did not result in a significant change in TfR retention. Next, we confirmed these results in primary macrophages. As observed for RAW264.7 macrophages, infection of human monocyte derived macrophages (HMDMs) with $Y$. pestis resulted in increased intracellular TfR intensity per cell per field, while infection with paraformaldehyde killed Y. pestis or E. coli had no impact on recycling (Fig.4-10 C,D,F \& G). Finally, HMDMs were also infected with Salmonella enterica Typhimurium to determine if infection with another intracellular pathogen impacted the host cell recycling pathway. In contrast to $Y$. pestis, S. enterica Typhimurium had no impact on TfR recycling (Fig. 4-10H \& I). Importantly, Y. pestis intracellular growth was also not impacted by the presence of the TfR antibody (Fig.4-11). Together these data demonstrate that $Y$. pestis actively inhibits host cell recycling (vs. killed Y. pestis), inhibition is not just a response by macrophages to bacteria (vs. E. coli K12), and inhibition is pathogen specific (vs. S. enterica Typhimurium).

\section{Stalling host cell recycling is required for $Y$. pestis replication}

Y. pestis infection stalls host recycling as early as $2 \mathrm{~h}$ post-infection. Since Rab11b is recruited to the $\mathrm{YCV}$, it is possible that $Y$. pestis infection stalls host cell recycling through sequestration of Rab11b on the YCV. If this is true then overexpression of Rab11b may be able to restore host cell recycling. To test this hypothesis, RAW264.7 macrophages transfected with a plasmid overexpressing wildtype Rab11b-EGFP [108] were infected with Y. pestis CO92 pCD1(-) pGEN::mCherry and TfR recycling was monitored. At $2 \mathrm{~h}$ post-infection untransfected cells showed 
a >2 fold increase in both TfR intensity and endosomes per cell over Rab11b transfected cells (Fig. $12 \mathrm{~A} \& \mathrm{~B})$. By $24 \mathrm{~h}$ post-infection Rab11b transfected cells have a 2 fold increase in TfR intensity (Fig. 4-12C), and have a significantly lower level of endosomes per cell ( $p \vee \leq 0.001$; Fig. 4-12D). In contrast, untransfected cells have a significantly higher TfR intensity per cell ( $p \vee \leq 0.01$; Fig. 4$12 \mathrm{C}$ ), but have a $>2$ fold increase in endosomes per cell (Fig. 4-12D). Overexpression of Rab11b restored host cell recycling and significantly decreased the amount of TfR endosomes per cell (pV $\leq 0.001$; vs untransfected). Our data showed Rab11b loss significantly impacted Y. pestis survival at $10 \mathrm{~h}$ post-infection ( $\mathrm{p} \leq 0.001$; Fig. 4-5D), and through accumulation of Rab11b Y. pestis stalled host cell recycling. We therefore, hypothesized that Rab11b overexpression would impact bacterial burden in Rab11b transfected cells. To test this, we quantified bacteria number per cell in Rab11b transfected vs untransfected cells. Overexpression of Rab11b prevents bacterial replication in RAW264.7 macrophages at $24 \mathrm{~h}$ (Fig. 4-12 E \& F). At $2 \mathrm{~h}$ post-infection there are equal numbers of bacteria between transfected and untransfected cells (Fig. 4-12F). However, at $24 \mathrm{~h}$ postinfection there $>4$ fold increase in bacteria in untransfected cells (Fig. 4-12H). These data demonstrate that $Y$. pestis infection disrupts host cell recycling and this is required for bacteria replication.

\section{Discussion}

Y. pestis is easily phagocytosed by monocytes/macrophages during the initial transition from the flea to the mammalian host [54]. Once engulfed $Y$. pestis resides in a Yersinia-containing vacuole (YCV) that is remodeled by the bacterium to avoid normal phagolysosome maturation and generate a cellular compartment for replication [54, 55, 59]. While paraformaldehyde-killed Y. pestis is trafficked to an acidified compartment, and live $Y$. pestis is not $[56,59,61,83,86]$. These observations indicate $Y$. pestis actively modulates host-pathogen interactions during infection to avoid YCV acidification and establish a replicative niche within the macrophage. While the fate of Y. pestis within the host macrophage is well studied, the mechanisms and cellular processes necessary to support $Y$. pestis intracellular infection are largely unknown.

To better understand the cellular processes required for $Y$. pestis intracellular survival, we designed and conducted the first ever RNAi genome-wide screen to identify essential host 
processes necessary for survival of $Y$. pestis inside macrophages. From our screen we identified 135 host targets required by $Y$. pestis during infection of the macrophage. These gene targets enriched for three key cellular processes: vesicular trafficking, transport, and localization within the cell. The ontologies of these cellular processes provide the evidence that $Y$. pestis specifically targets host vesicular trafficking during intracellular infection. Analysis of these GO ontologies revealed overlap between the processes which highlighted the essential role of the host recycling pathway for the dynamic maturation of the YCV. Furthermore, mapping the interactions between the validated and primary screen hits showed enrichment focused on recycling Rab GTPases.

Following up on these findings, we demonstrate that $Y$. pestis recruits recycling markers Rab4a and Rab11b to the YCV to resemble/enter the recycling pathway. RNAi of Rab4a, Rab11b, and Myo5b increased YCV acidification, but two distinct phenotypes were observed. Rab4a knockdown increased YCV acidification within 20 mins post-infection, which was sustained out to 80 mins, whereas, inhibition of Rab11b and Myo5B did not have a dramatic impact on YCV acidification until 80 mins post-infection, and the impact was not to the same extent as Rab4a. From this data, we postulate that Rab4a and Rab1b (identified to be required for rapid inhibition of YCV acidification in a previous study [61]) are recruited to the YCV specifically to arrest YCV acidification and avoid phagolysosome maturation.

Conversely, Rab11b while recruited early to the YCV, plays a dominant role in bacterial replication and is not specifically required to avoid YCV acidification. Rapid recycling via Rab4a occurs off of the early endosome prior to trafficking into a slow recycling arm, which requires Rab11b [238]. Rab11b moves recycling endosomes into the slow recycling pathway through additional trafficking components, such as Myo5b, Rab11fip proteins and Arf6 [238, 243, 244]. Our findings suggest that $Y$. pestis is trafficked into a slow endocytic path as RNAi inhibition of Rab11b and Myo5b have no impact on bacterial survival within $2 \mathrm{~h}$ of $Y$. pestis infection of the macrophage. However, by 10 h post-infection both Rab11b and Myo5b attenuate bacterial burden by $>50 \%$. Additional analysis of the limited, but growing literature on recycling endosome maturation, we identified additional genes that regulated Rab11b also inhibited Y. pestis survival. Known Rab11b interactors are: EHD1, Rab11fip2, 3, \& 5, and Arf1 [238]. Two of these components, (EHD1 \& 
Arf1), inhibited $Y$. pestis survival by $\sim 30 \%$ at $10 \mathrm{~h}$ during primary screening, but did not make cutoff criteria for inclusion in the validation screens. We believe Rab11b and Myo5b are essential for latter stages of YCV biogenesis, potentially through exploitation of the slow endocytic recycling arm. Furthermore, our novel finding that overexpression of Rab11b prevents $Y$. pestis replication suggests that targeting Rab11b is a major regulator separating intracellular $Y$. pestis survival from replication within a spacious YCV.

Intriguingly, inhibition of Rab11 isoforms have been shown to impact the survival of other pathogens that replicate in expanded independent intracellular compartments, such as Anaplasma phagocytophilum and Chlamydia spp [191, 194]. A. phagocytophilum recruits Rab11a to the Anaplasma phagocytophilum-occupied vacuole (ApV) within $5 \mathrm{~h}$ post-infection ( $>43 \%$ of $\mathrm{ApVs}$ ), and this is maintained out to $24 \mathrm{~h}$ post-infection [191]. Inhibition of Rab11a, using a dominant negative form which cannot tether to the $\mathrm{ApV}$, they attenuate $A$. phagocytophilum intracellular survival by $>10 \%$ at $5 \mathrm{~h}$ post-infection in HL-60 cells [191]. RNAi knockdown of Rab11a in HeLa and HEp-2 cells, prevents Chlamydia trachomatis reversion from the metabolically active reticulate body into the inert infectious elementary body by blocking the bacteria's ability to disrupt the Golgi [245-247]. Excitingly, when we re-visited the Coxiella burnetii genome-wide RNAi screen data (another pathogen residing in a pathogen containing vacuole termed the CCV) we noticed that inhibition of Rab11b also prevented bacterial replication [131]. While to our knowledge the role of Rab11b in C. burnetii has not been fully defined, one suspects it is also recruited to the CCV. This is suggestive that exploitation of Rab11 isoforms is potentially a specific requirement of pathogens that expand their containing vacuoles during bacterial replication. Additional, studies to define the role of Rab11 in vacuole expansion would shed substantial light on how these pathogens acquire the machinery to support vacuole expansion.

Moreover, we show $Y$. pestis infection stalls the host cell recycling by sequestration of Rab11b, making this the first identification of any pathogen to arrest endocytic recycling traffic. While studies have shown various pathogens manipulate host Rab GTPase machinery for survival (for review [102]), no one has demonstrated a living bacterium capable of stalling an entire trafficking pathway. Recently, studies using Anthrax toxin demonstrate disruption of host recycling 
endosomes was directly attributed to the toxins impact on Rab11b and Sec15 exocyst assembly [248]. However, through overexpression of Rab11b they were able to restore wildtype Drosophila wing structure and normal recycling [248]. Within this context, we were intrigued that during our screen Arf6 RNAi, a well-studied Rab4 effector and a negative regulator of rapid recycling [249253], had a slight hypervirulent phenotype at $10 \mathrm{~h}$ post-infection This observation could be suggestive, that inhibition of rapid recycling forces cellular compensation by the slow recycling arm and Rab11b through an increase in slow recycling endosomes. Due to this, there is less total free unbound Rab11b available for the cell to manage trafficking of these recycling endosomes, which in turn lowers the threshold needed to be sequestered to stall efficient recycling within the cell, and thusly, a hypervirulent phenotype for $Y$. pestis as more bacteria are capable of establishing a replicative niche. Studies with Anthrax toxin and our $Y$. pestis infection data suggest there are quantifiable finite protein levels for each Rab GTPase within the cell, and disruption/retention of a specific Rab GTPase dilutes the unbound pool available for cellular processes. If true, this would provide a novel insight into general intracellular pathogenesis, as a pathogenic organism, whether it be virus, parasite or bacterial, target host cell Rab GTPases for survival and actively force the infected cell to compensate to the benefit of the pathogen. Through this compensation the infectious agent could create a diversion hiding their presence to the innate host defenses.

Looking deeper into a mechanistic explanation for overexpression of Rab11b inhibition of Y. pestis replication we noticed recent findings linking Rab11b to autophagy [254]. Szatmari and colleagues, demonstrate that Rab11b interacts with Hook, a negative regulator of endosome maturation, to facilitate crosstalk between recycling endosomes and induction of autophagy [254]. This peaked our interested as studies have noted that latter stages ( $>8 \mathrm{~h}$ post-infection) of $Y$. pestis replication occurs within a spacious YCV that resembles an autophagosome as it acquires both LC3-II and double membranes [88, 89]. Though the significance of autophagy to $Y$. pestis survival/replication during infection is unknown, findings in $Y$. pseudotuberculosis, the closet ancestral relative within the Yersinia genus, suggest that autophagy is required for bacterial replication $[88,89]$. Ligeon et al., demonstrate $Y$. pseudotuberculosis acquires VAMP3 within 30 mins post-infection and is exchanged for VAMP7 within 3 to 24 hours, and these VAMP3 and 7 
dynamics are required for YCV LC3-II single/double membrane formation [89]. Studies by Moreau and colleagues further show that $Y$. pseudotuberculosis replicates in autophagosomes, and induction of autophagy with rapamycin significantly increased metabolically active bacteria [88]. Additionally, these studies show that Atg4b C74A (enzymatically inactive Atg4b) prevented LC3 recruitment to $Y$. pseudotuberculosis YCV [88]. In this context, our lab observed macrophage infection using phoPQ mutants of $Y$. pestis, compensated for bacterial number, survive the first 8$10 \mathrm{~h}$ post-infection, have no significant difference in YCV acidification, and the decline in bacterial burden coincides with wildtype replication (MC \& ARP unpublished data). Transcriptional studies of $\triangle p h o P Q$ mutants indicate the stress response regulator controls $188 Y$. pestis genes [86]. The majority of these differentially regulated genes are putative or hypothetical, but appear to be involved with Y. pestis' ability to acquire/sense nutrient deprivation and deal with antimicrobial peptides [86]. These studies further conclude that that phoPQ does not impact trafficking of the YCV but adaption of the YCV to a replicated niche [86]. In either case, the link between Rab11b and autophagy induction is exciting as we show $Y$. pestis stalls host recycling and restoration of this process through overexpression of Rab11b prevents bacterial replication. Perhaps, Y. pestis stalls host cell recycling to facilitate entry into autophagosomes, or that autophagy triggers mammalian specific virulence factors for $Y$. pestis. In this sense, one would hypothesize that overexpression of Rab11b would also prevent $Y$. pseudotuberculosis replication as well - provided the end result of stalling host recycling is to induce autophagy within infected cells.

In summary, we completed a genome-wide RNAi HTS to identify required host factors to support $Y$. pestis intracellular survival. From our data, we have demonstrated for the first time that Y. pestis remodels the YCV to resemble a recycling endocytic vacuole, and through the sequestration of Rab11b stalls host recycling. We further show that overexpression of Rab11b prevents bacterial replication. Our overexpression data demonstrates for the first time an exploited host protein that differentially separates an intracellular pathogen's survival from replication. Our future goals include defining the role of Rab11b and inhibition of host cell recycling in the context of autophagy and defining the mechanisms used by $Y$. pestis to generate the YCV. 


\section{Materials and Methods:}

Bacterial strains, plasmids, siRNA and transfection of macrophages. All bacterial strains listed in table 4-1. Y. pestis CO92 [9] pCD1(-) and KIM D-19(pgm $\left.{ }^{(-)}\right)$(BEI Resources NR-4681) were cultivated at $26^{\circ} \mathrm{C}$ in Brain Heart Infusion (BHI) broth (Difco). E.coli and S. typhimurium was cultivated at $37^{\circ} \mathrm{C}$ in Luria-Bertani (LB) broth (Difco). Growth media supplemented with Carbenicillin at $50 \mu \mathrm{g} / \mathrm{mL}$ when needed. Bioluminescent derivatives were generated using the LuXPtolc bioreporter as described previously [151]. Y. pestis and E. coli fluorescent derivatives bacterial strains are described previously [61]. S. enterica Typhimurium pGEN-PEM7::DsRED was generated using Eppendorf protocol \# 4308 915.532. RAW264.7 macrophages were obtained from ATCC and cultured in DMEM, $100 \mathrm{mM}$ glucose $+10 \%$ FBS (Hyclone). For 96-well RNAi highthroughput screening, RAW264.7 macrophages were forward transfected using Tecan liquid handling robotics (Tecan) to pool 3 siRNAs from the Silencer siRNA Mouse Genome Library v3 (Ambion) at $[1 \mu \mathrm{M}]$ final concentration in $20 \mu \mathrm{l}$ Opti-MEM, and then mixed with $10 \mu \mathrm{l}$ of $0.03 \%(\mathrm{v} / \mathrm{v})$ Lipofectamine RNAiMax/Opti-MEM (Life Technologies) as described by the manufacturer. $30 \mu \mathrm{l}$ of the siRNA-Lipofectamine complex was added to each well of a white flat-bottom 96-well plate (Greiner), incubated at room temperature for $10 \mathrm{~min}$, prior to addition of $1 \times 10^{4}$ RAW264.7 macrophages suspended in $80 \mu \mathrm{l}$ of DMEM $+10 \%$ FBS. 24-well plates reagents were increased 4-fold previously described [61]. For plasmid transfection, 4.4 x 105 RAW264.7 macrophages were transfected using $0.5 \mu \mathrm{g}$ of plasmid with JetPrime (Polyplus) as described by the manufacturers. All single siRNAs used are in Table 4-2.

gRT-PCR, Western blot and Cell Viability. Rab GTPase expression, total RNA from $1.6 \times 10^{5}$ transfected cells was isolated as previously described [61] and qRT-PCR was performed using SybrGreen (Life Technologies) with primers in Table 4-3. Relative expression was calculated using $\Delta \Delta \mathrm{Ct}$ method [228]. Western blot was performed as previously described [61]. For AlamarBlue (Life Technologies) cell viability, $10 \mu \mathrm{l}$ reagent was added directly to macrophages and incubated at $37^{\circ} \mathrm{C} 5 \% \mathrm{CO}_{2}$ for $2 \mathrm{hrs}$, then read using Synergy 4 (ex: 560: Em: 600). 
Bacterial infection of macrophages. Macrophages were infected with $Y$. pestis as previously described $[59,61,151]$. In brief, bacteria cultivated overnight at $26^{\circ} \mathrm{C}$ in $\mathrm{BHI}$, washed in PBS, and diluted in prewarmed DMEM+10\%FBS. Bacteria were added to macrophages and infection synchronized by a $200 x g$ centrifugation for 5 mins. 20 mins post infection extracellular bacteria were killed with gentamicin $(16 \mu \mathrm{g} / \mathrm{mL})$ for one hour, and then media was replaced with DMEM + $10 \%$ FBS containing $2 \mu \mathrm{g} / \mathrm{mL}$ gentamicin for the duration of the experiment. Intracellular $Y$. pestis was quantified using a Synergy HT or Synergy 4 plate reader (Biotek; $1 \mathrm{sec}$ read with sensitivity set at 150 ) or conventional bacterial enumeration as described previously [151]. S. enterica Typhimurium infection of macrophages was done with 200xg centrifugation for 10 mins. 60 mins post infection extracellular bacteria were killed with gentamicin $(100 \mu \mathrm{g} / \mathrm{mL})$ for one hour, and then media was replaced with DMEM $+10 \%$ FBS containing $10 \mu \mathrm{g} / \mathrm{mL}$ gentamicin for the duration of the experiment.

Bioinformatic analysis. Validated and primary screen hits were stored with both Entrez Gene and MGI identifiers. A script was made to pull interacting partners from all experimental evidence codes from BioGRID [255] and STRING [256] databases using MGI and Entrez Gene identifiers. The script stored interactors for the input datasets, validated (135 genes) and primary hits (364 genes), as follows: 1) direct interactions within each individual dataset, and 2) direct interactions from validated to primary hits. These interactions were stored and imported into Cytoscape (v3.30; [257]) to generate interaction maps. For GO Ontology clustering, the validated (135) hits were imported by Entrez Gene identifier to Cytoscape plugins ClueGO [237] and CluePedia [236]. Genes were clustered using all GO evidence codes, a minimum kappa score of 0.4 and a $p$-value threshold of 0.05 . To construct Circos plots, individual gene GO ontologies for validated datasets were generated using Entrez Gene identifier in PANTHER [258]. Circos plots were then overlaid with screening score data and interactions previous stored from BioGRID and STRING databases.

\section{$\underline{T f R}$ recycling and Acidification assay, Immunofluroescent staining, and confocal microscopy}

For confocal microscopy, cells were fixed to coverslips with $2.5 \%$ paraformaldehyde for 30 min. Indirect immunofluorescent staining, fixed cells were blocked with 3\% BSA overnight and incubated with rabbit anti- $Y$. pestis serum $(1: 1,000)$. Unbound primary antibodies were removed by washing 
and anti-rabbit Alexa Fluor 594 secondary antibody (1:2000; Life Technologies). For TfR recycling assay using siRNA transfection, $1.6 \times 10^{5}$ were transfected as described above in a 24 -well plate (Greiner) and incubated either for $48 \mathrm{~h}$ at $37^{\circ} \mathrm{C} 5 \% \mathrm{CO}_{2}$. Prior to kinetic assay using siRNA transfection, cells were washed three times in cell culture grade PBS (Hyclone), left in cell culture PBS and placed on ice for 30 mins. Media was aspirated and replaced with $37^{\circ} \mathrm{C}$ pre-warmed DMEM, $100 \mathrm{mM}$ glucose $+10 \%$ FBS (Hyclone) with anti-TfR (1/1000 dilution; Abcam ab84036). Cells were moved to $37^{\circ} \mathrm{C}$ waterbath. At desired time points coverslips were removed and placed in acidic stripping buffer (HBSS $+50 \mathrm{mM}$ glycine $+150 \mathrm{mM} \mathrm{NaCl}+0.2 \% \mathrm{BSA} \mathrm{pH} 4$ ) on ice. Coverslips were washed thrice with acidic stripping buffer and fixed with $2.5 \%$ paraformaldehyde for $15 \mathrm{~min}$ at room temperature. Cells were rocked in permeabilization buffer $(0.5 \%$ Tween $20+$ $3 \% \mathrm{BSA})$ overnight at $4^{\circ} \mathrm{C}$ prior to $1 \mathrm{~h}$ incubation with staining buffer $(0.5 \%$ Tween $20+3 \% \mathrm{BSA}+$ anti-rabbit 2,000 Alexa Flour 488). For TfR recycling during bacterial infection, $1 \times 10^{5}$ RAW264.7 macrophages were seeded in a 24-well plate (Greiner) and incubated overnight at $37^{\circ} \mathrm{C} 5 \% \mathrm{CO}_{2}$. Macrophage media was replaced with $37^{\circ} \mathrm{C}$ pre-warmed DMEM, $100 \mathrm{mM}$ glucose $+10 \%$ FBS (Hyclone) with anti-TfR (1/1,000 dilution; Abcam ab84036). Bacterial strains were diluted to desired MOls in DMEM, $100 \mathrm{mM}$ glucose $+10 \%$ FBS (Hyclone) and added to macrophages. After $1 \mathrm{~h}$ gentamicin killing media was replaced with appropriate gentamicin maintenance doses with 1/1,000 anti-TfR antibody. Coverslips were removed at 2 and $24 \mathrm{~h}$ post-infection, placed in acidic stripping buffer and processed as described above. Lysotracker Red DND-99 (Life Technologies) acidification experiments were done as previously described [61]. For confocal microscopy, cells were fixed to coverslips and imaged as previously described [61]. All Coverslips were mounted with Prolong Gold with DAPI (Life Technologies),

and imaged on a Zeiss LSM 710 laser confocal microscope. Colocalization of Lysotracker Red DND-99 or proteins to the YCV was determined using the Coloc function in Imaris image analysis software (BitPlane). Quantification of TfR recycling was done in Fiji [259].

Statistics. Unless otherwise noted in corresponding figure legend, all data are shown as mean and standard error margin (SEM) of one representative experiment. All experiments were repeated three to six for biological trend and each experiment was repeated three times to confirm the 
phenotypes. For microscopy, at least 50 YCVs or 25 fields per biological replicate and experiment were analyzed. P-values calculated by one-way ANOVA or Student's T-Test, with appropriate posthoc testing when necessary using GraphPad Prism software 
Figures and Figure Legends

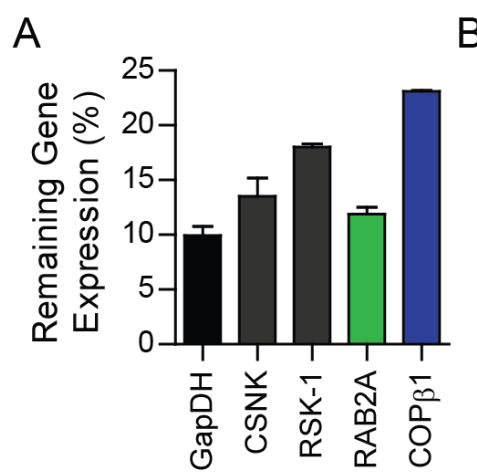

B

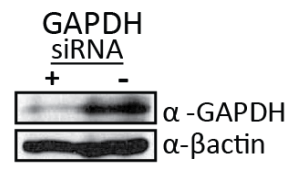

C

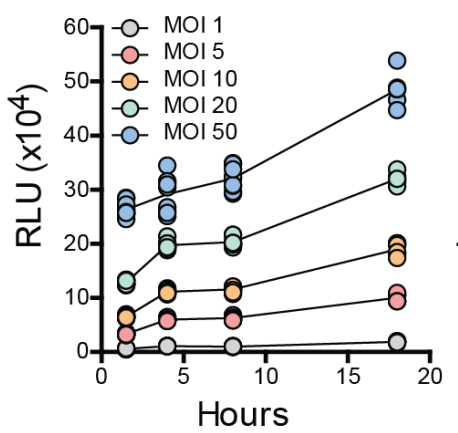

D

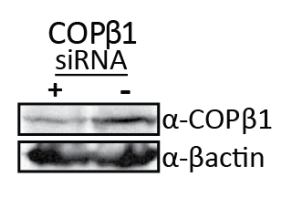

E
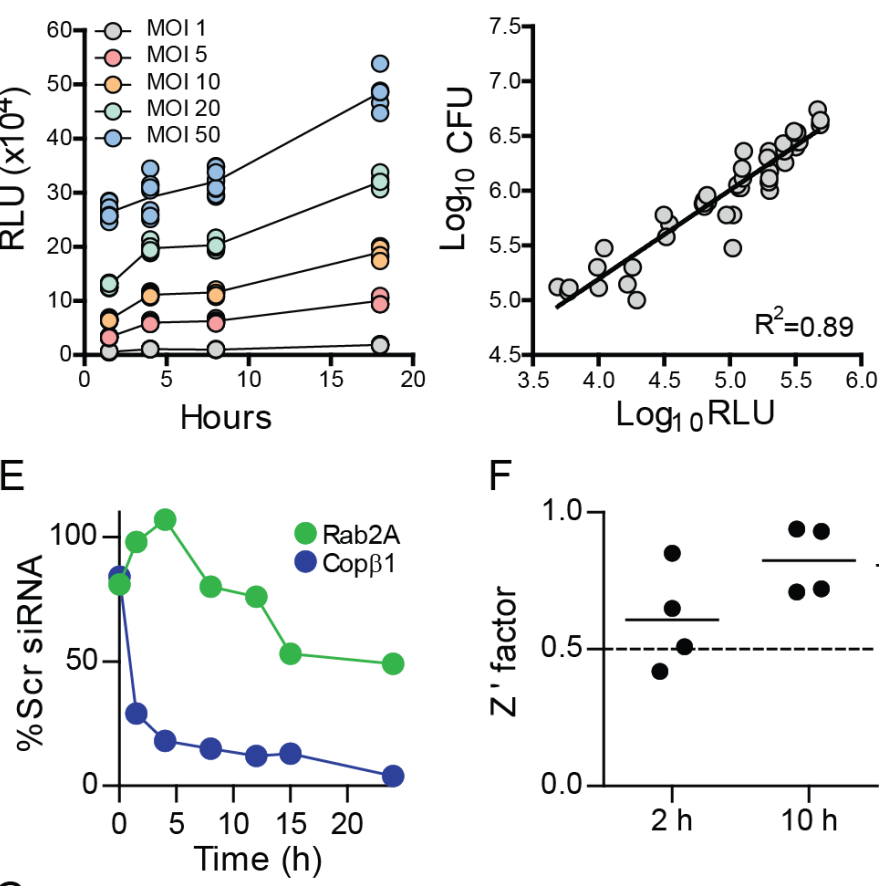

$G$

Overview of High Throughput Assay

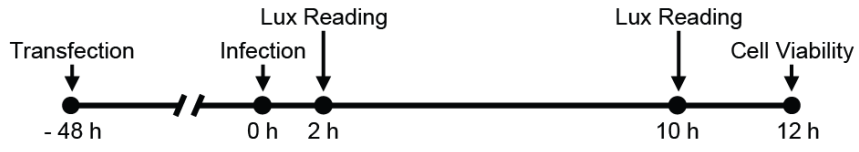


Figure 4-1. RNAi-based assay to identify host factors required for $Y$. pestis intracellular survival.

(A and B) To determine if reproducible RNAi could be achieved in RAW264.7 macrophages, cells were reverse transfected with siRNAs targeting indicated genes. $48 \mathrm{~h}$ post-transfection, cells $(n=3)$ were harvested for (A) RNA isolation and qRT-PCR (data represents the level of gene expression compared to scrambled siRNA control) or (B) protein isolation for Western blot analysis (Bactin was used as a loading control). (C and D) To demonstrate that the Y. pestis CO92 pCD1(-) LuXPtolC bioreporter accurately represents intracellular bacterial numbers, RAW264.7 macrophages were infected with Y. pestis CO92 pCD1(-) LuXPtolc at indicated MOIs $(n=12)$ and extracellular bacteria were killed with gentamycin. $(C)$ Bioluminescence $(R L U)$ of intracellular bacteria was determined at $1,4,8$ and $18 \mathrm{~h}$ post-infection. (D) At $18 \mathrm{~h}$, cells from each $\mathrm{MOI}(\mathrm{n}=3)$ were lysed and bacterial numbers (CFU) were determined and compared to $18 \mathrm{~h}$ bioluminescence (RLU). (E) To demonstrate that RNAi targeting specific genes could impact $Y$. pestis intracellular survival, RAW 264.7 macrophages were transfected with siRNAs targeting Rab2A or COP $\beta 1.48 \mathrm{~h}$ posttransfection macrophages were infected with Y. pestis CO92 pCD1(-) LuXPtolc (MOI 10). Extracellular bacteria were killed with gentamycin and intracellular bacterial bioluminescence monitored over time. Data is represented as percent RLU of scramble siRNA (scr) control. (E) To demonstrate robustness of the assay, RAW264.7 macrophages $(n=48)$ were reverse transfected with either scrambled siRNA (negative control) or siRNA targeting Copß1 (positive control). $48 \mathrm{~h}$ posttransfection macrophages were infected with $Y$. pestis CO92 $\mathrm{pCD} 1^{(-)}$LuXPtolc (MOI 10). Extracellular bacteria were killed with gentamycin and intracellular bacterial bioluminescence was determined at 2 and $10 \mathrm{~h}$ post-infection. Z' factor from 4 independent experiments are shown (bar = mean). (G) Overview of optimized high throughput assay for RNAi screening. 
A Primary Screen $(17,370$ total genes)

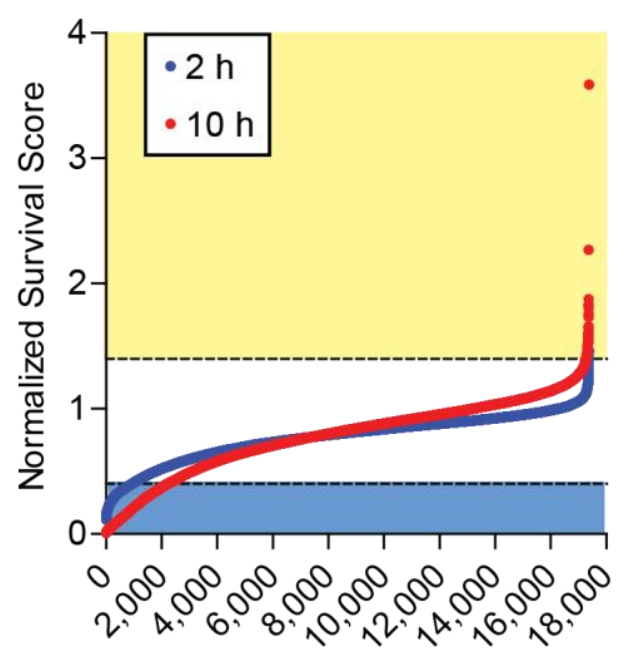

B

Secondary Screen (364 total genes)

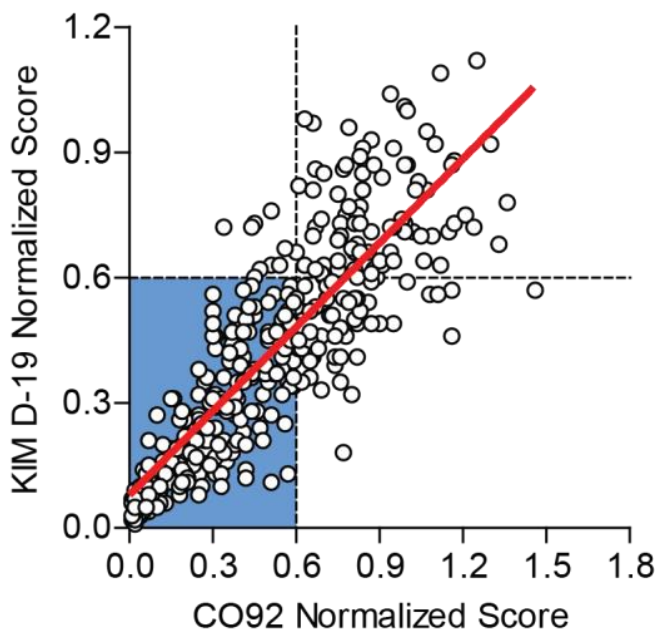

Figure 4-2. Identification of host factors required for Y. pestis intracellular survival

To identify host factors required for $Y$. pestis to survive in macrophages, RAW264.7 macrophages were reverse transfected with siRNAs for $48 \mathrm{~h}$. (A) Transfected cells were infected with Y. pestis CO92 $\mathrm{pCD} 1^{(-)}$LuXPtolc $(\mathrm{MOI} 10)$ and intracellular bacteria bioluminescence (RLU) determined at 2 (blue) and 10 (red) h post-infection. RLU values were normalized to controls [(gene RLU / avg. positive control RLU) / (avg. negative control RLU/ avg. positive control RLU)] and ranked from lowest to highest. Normalized scores $\leq 0.5$ are highlighted in blue and $\geq 1.4$ in yellow. (B) For secondary validation, transfected cells were infected with Y. pestis CO92 pCD1(-) LuXPtolC or KIMD19 pCD1(+) LuXPtolc (MOI 10) and intracellular bacteria bioluminescence (RLU) determined at $10 \mathrm{~h}$ post-infection. RLU values were normalized to controls and compared to each other. The red line represents the line of best fit (linear regression). Normalized scores $\leq 0.6$ are highlighted in blue. 


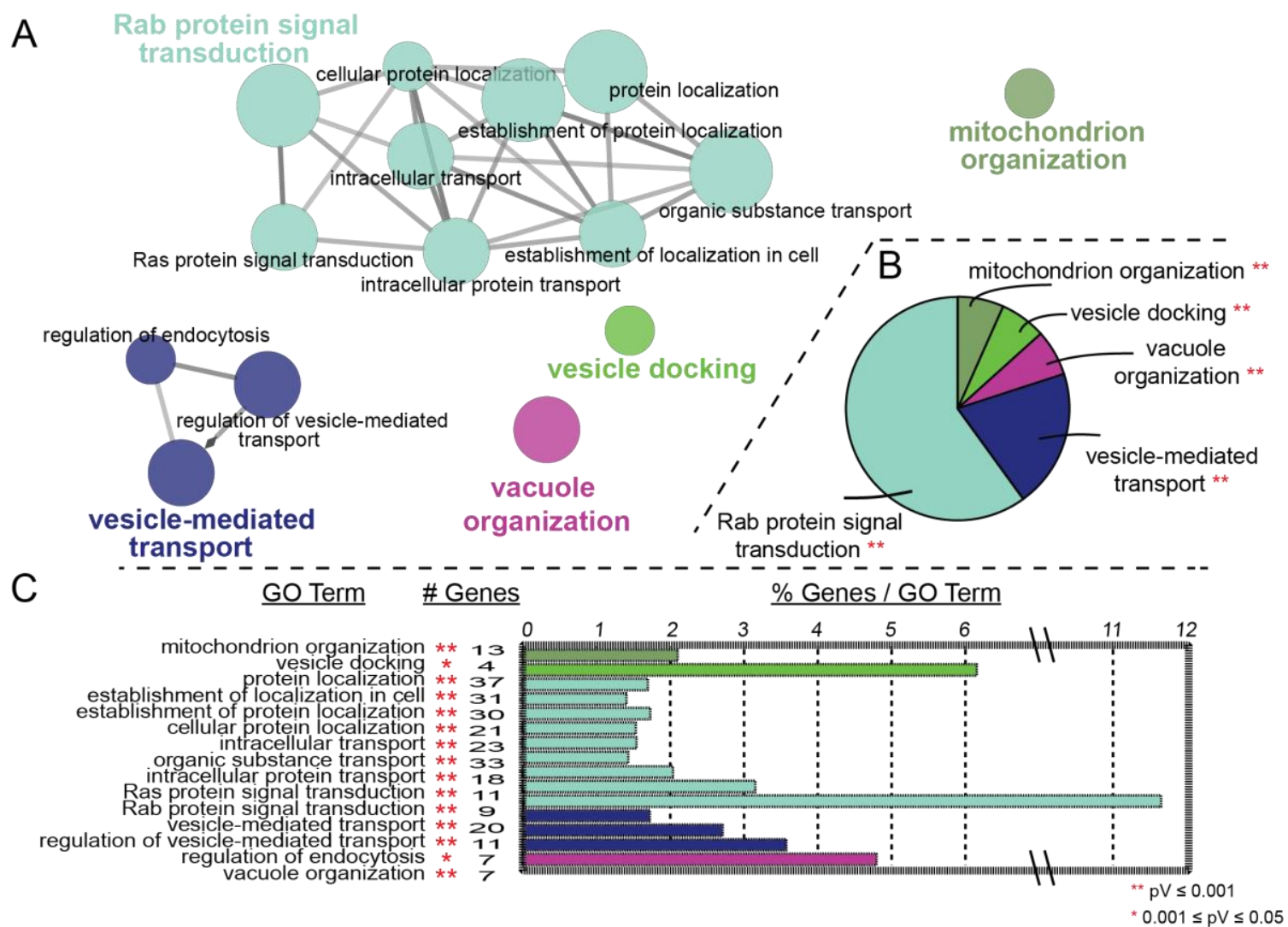

Figure 4-3. ClueGO clustering of validated genes impacting Y. pestis intracellular survival.

(A) Cytoscape generated layout for GO term node clusters. Clusters are color coded by highest order parent ontology. (B) Pie chart representing the percent parent ontology represented as a whole within our validated genes. (C) Detailed ontologies within the parent clusters broken down by the number of genes and the percent representation of those genes within the detailed term. 
A

Rab GTPase direct interactions within validated hits

B

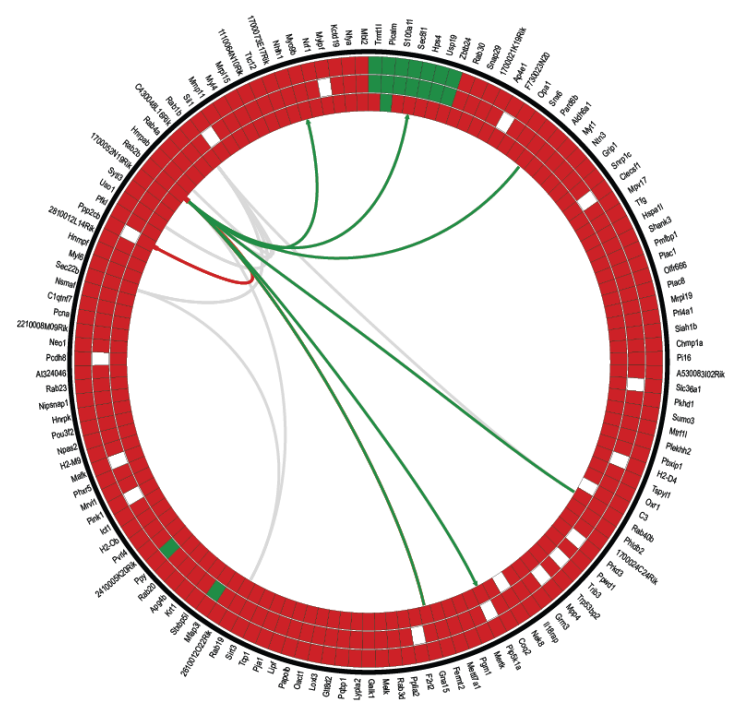

Direct interactions between validated and primary hits

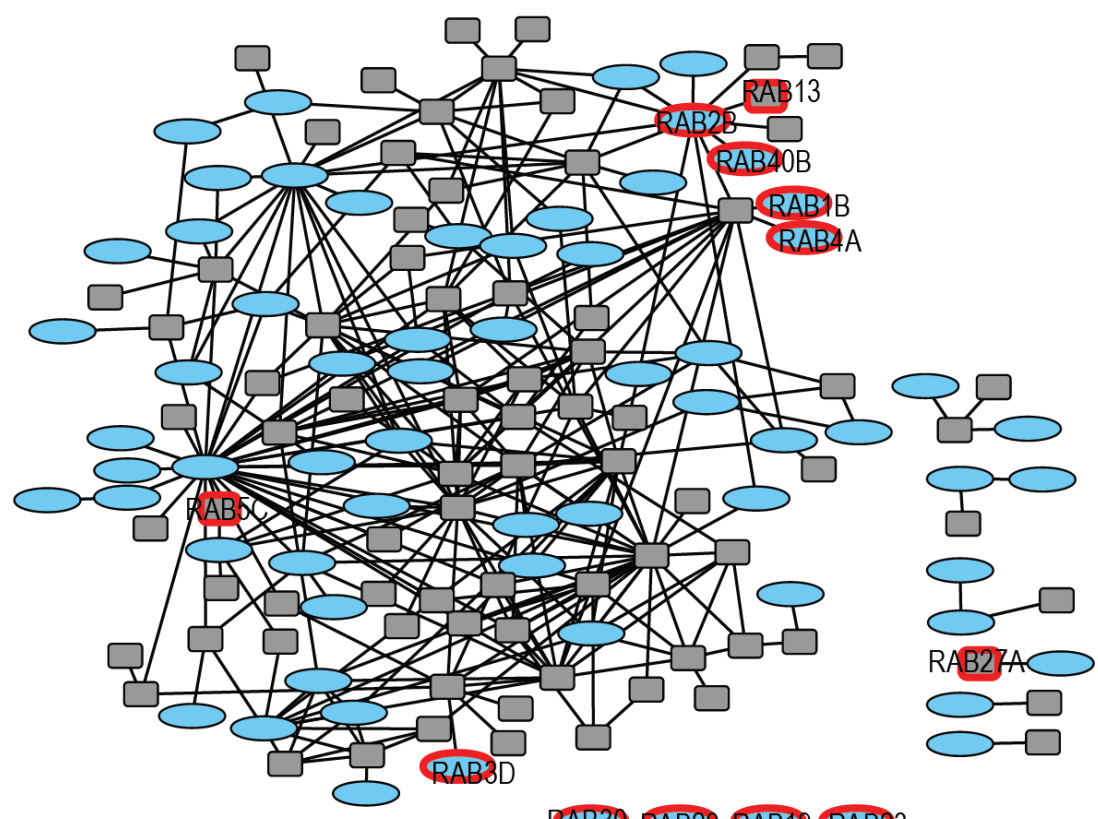

RAB30 RAB20 RAB10 RAB23

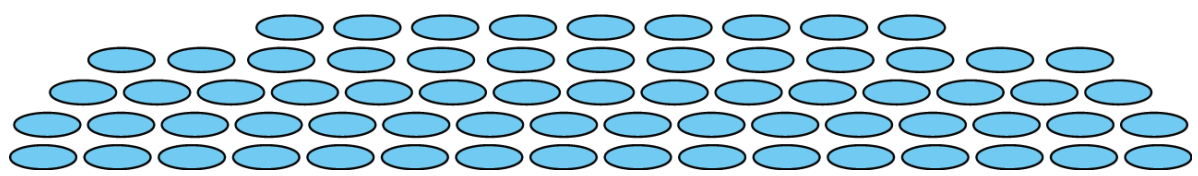


Figure 4-4. Identification of Rab GTPase interactions within primary screen hits.

(A) To determine the Rab GTPases network interactions, we pulled all interactions from the Rab GTPases to the validated screen hits $(n=135)$. The resulting interactions are displayed as a Circos plot as follows. Lines: Gray (interaction), Green (activator) and Red (inhibitor). Each square represents if the target gene met cutoff criteria for inhibition (red) or hypersurvival (green) in the primary (outside ring), CO92 secondary (middle ring) or KIM-D19 secondary (inside ring) screens. Gene symbols are on the outside. (B) Interactome of direct interactions between primary and validated hits. Gray squares are primary hits, blue ovals are validated genes. Rab GTPases are highlighted with red boarder and labeled 
A $\quad$ QRT-PCR Relative Expression B siRNA Cytotoxicity
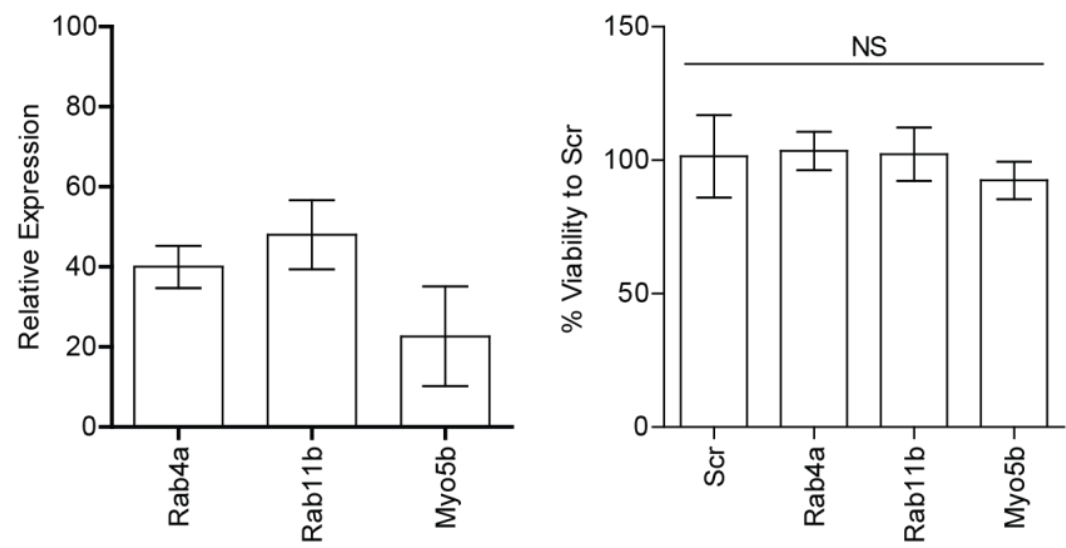

C

$2 \mathrm{hr}$ RLU post-infection

D

$10 \mathrm{hr}$ RLU post-infection
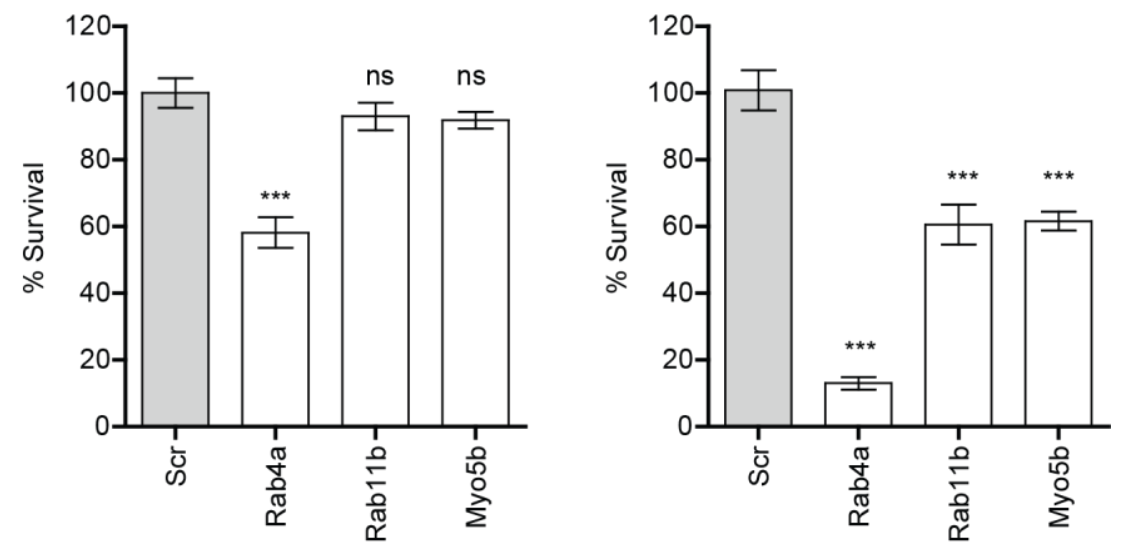

E $\quad 10 \mathrm{hr}$ CFU post-infection

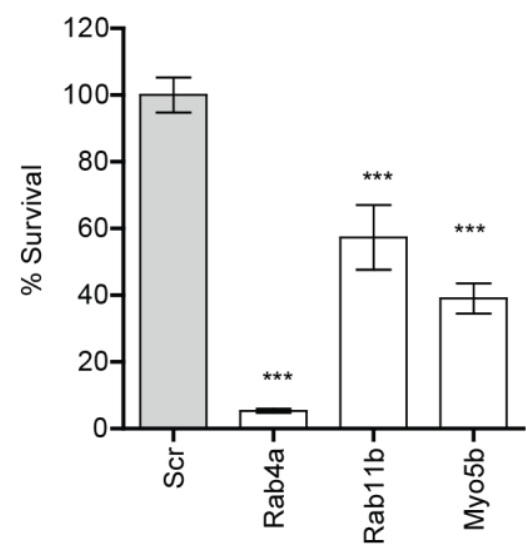




\section{Figure 4-5. Host recycling inhibits $Y$. pestis intracellular survival}

RAW 264.7 macrophages were transfected with a single siRNA targeting Rab4a, Rab11b or Myo5B. At 48 h post-transfection (A) RNA samples were harvested for qRT-PCR ( $n=9)$ or (B) cell viability was determined $(n=5)$. To determine the impact of RNAi on $Y$. pestis survival, transfected RAW264.7 macrophages $(n=8)$ were infected with Y. pestis CO92 pCD1(-) LuXPtolc $(\mathrm{MOI} 10)$ and intracellular bacterial numbers were determined by bioluminescence at (C) $2 \mathrm{~h}$ or (D) $10 \mathrm{~h}$ postinfection and compared to scrambled (scr) controls. (E) At $10 \mathrm{~h}$ post-infection, a subset of samples $(n=3)$ were harvested for convention bacterial enumeration. Statistical significance was calculated by one-way ANOVA with Tukey's Multiple Comparison post hoc. ${ }^{* * *}=p \leq 0.001$; ns $=$ not significant. 
A

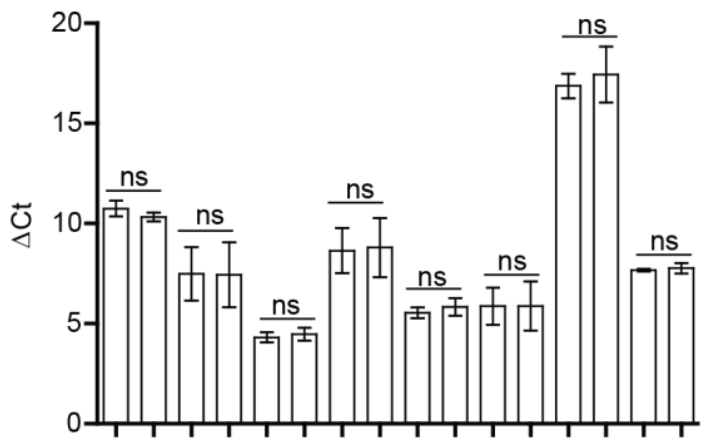

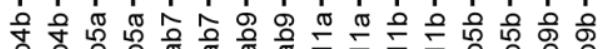

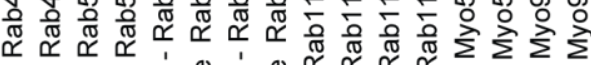

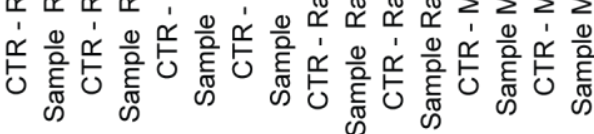

$\mathrm{B}$

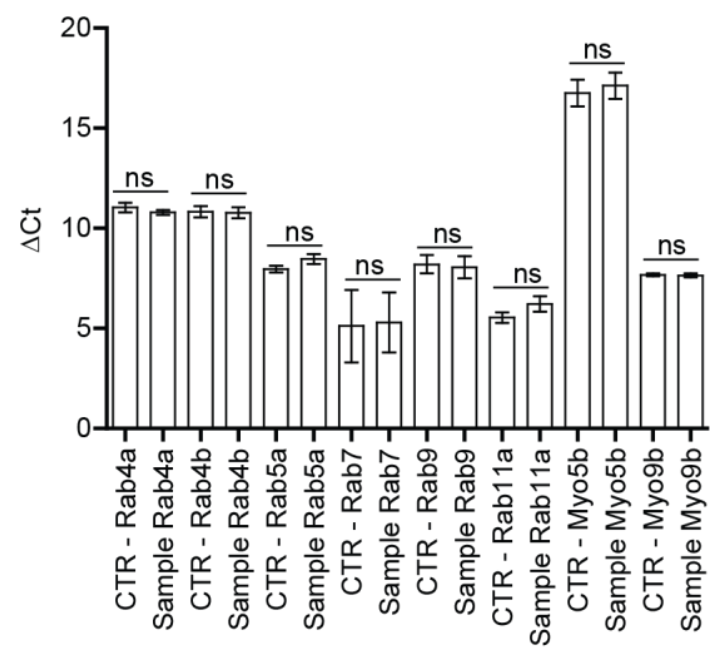

Figure 4-6. RNAi inhibition of Rab4a and Rab11b does not impact the phagolysosome

RAW 264.7 macrophages were reverse transfected for $48 \mathrm{~h}$ with single siRNA targeting indicated host genes. Total RNA was isolated after $48 \mathrm{~h}$ and $\triangle \mathrm{CT}$ (avg. target gene - avg. reference gene) determined for gene targets in either Rab4, Rab11b or scrambled negative control siRNA. (A) $\triangle C T$ of targets in Rab4a treated vs scrambled negative control (CTR; $n=9$ ). (B) $\triangle C T$ of targets in Rab11b treated vs scrambled negative control (CTR; $n=9)$. 
A

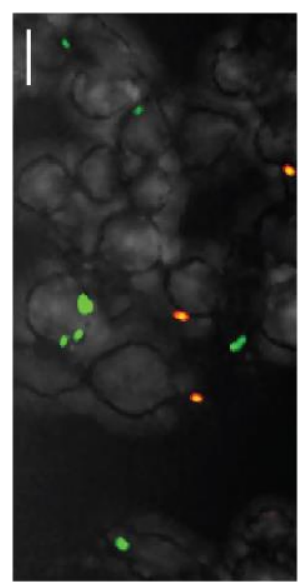

C
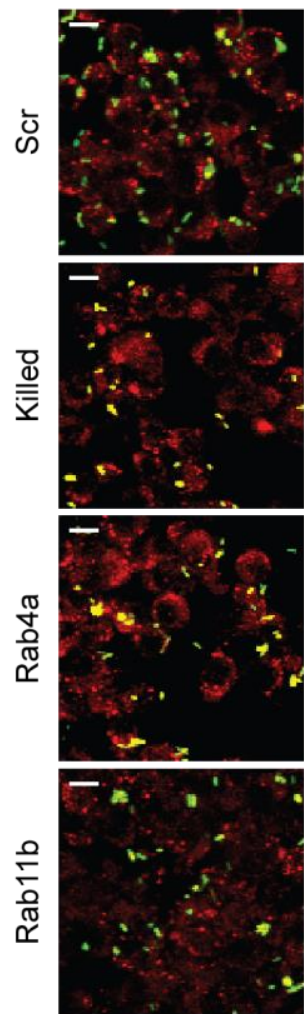

ํㅡㄹ

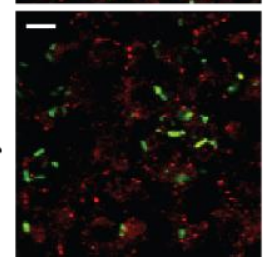

B

20 min post-infection

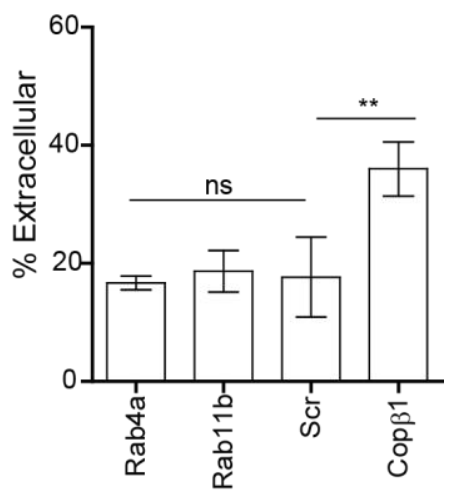

D

20 min post-infection

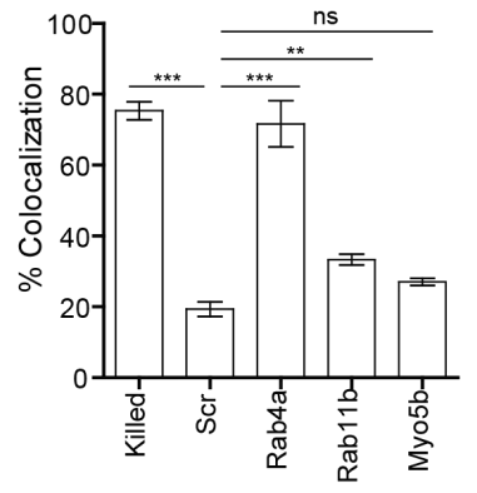

E

80 min post-infection

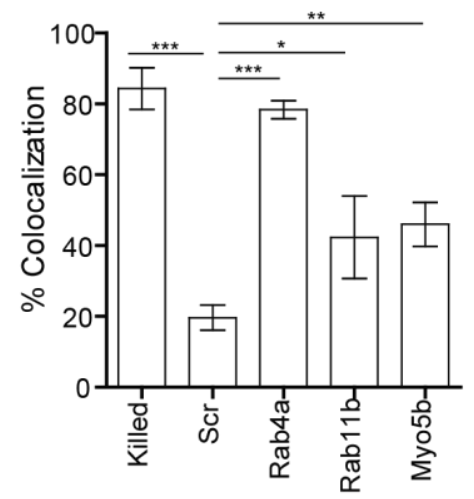


Figure 4-7. Rab4a of the recycling pathway is essential for $Y$. pestis to avoid the phagolysosome

For invasion, cells were infected with $Y$. pestis CO92 pCD1(-) pGEN222 EGFP (MOI 3). After 20min infection cells were fixed, and counter stained by indirect immunofluorescence with anti- $Y$. pestis antibody. (A) Representative image of differential staining showing intracellular bacteria (green) and extracellular bacteria (red/yellow). 10 $\mathrm{mm}$ scale bar. (B) Percent of extracellular bacteria at $20 \mathrm{~min}$ post-infection, represented as mean $\pm \mathrm{std}(\mathrm{n}=3)$. After transfection cells were incubated with Lysotracker Red DND-99 for 1hr. After $1 \mathrm{hr}$ cells were infected with either live Y. pestis CO92 pCD1(-) pGEN222 EGFP or paraformaldehyde killed Y. pestis CO92 pCD1(-) pGEN222 EGFP (MOI 3). Colocalization determined by confocal microscopy. (C) Representative field images at $20 \mathrm{~min}$ post-infection. 10 $\mu$ m scale bar. (D \& E) Percent colocalization with Lysotracker Red DND-99 at 20 and 80 min post-infection, represented as mean \pm std $(n=3)$. Significance calculated by one-way ANOVA. ns $=$ not significant, ${ }^{*}=p V \leq 0.05,{ }^{* *}=p V \leq 0.01,{ }^{* * *}=p V \leq 0.001$ 


\section{A}
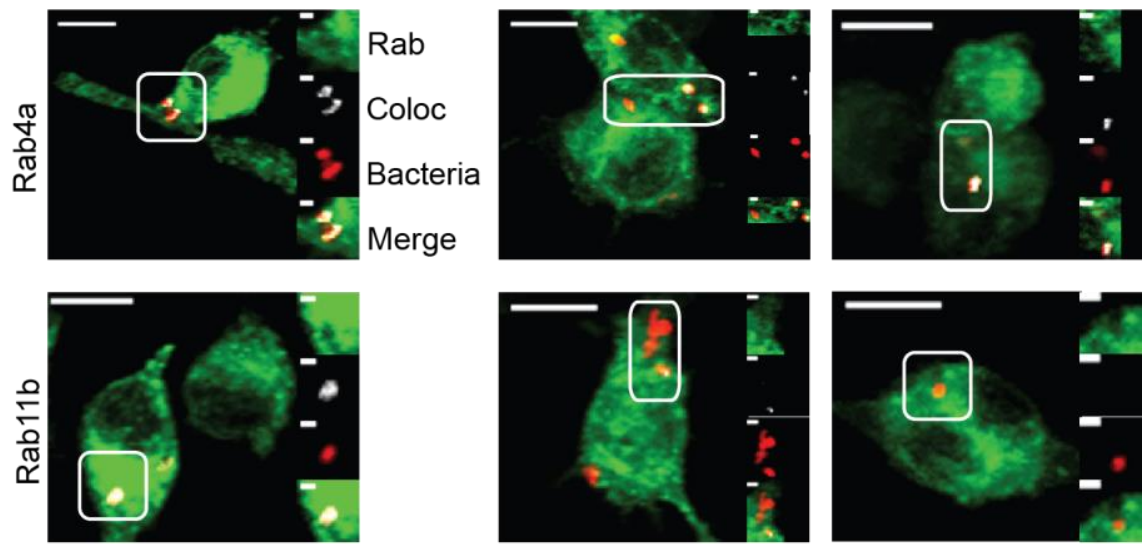

Live

Kill

E. coli

Y. pestis

B

Rab4a

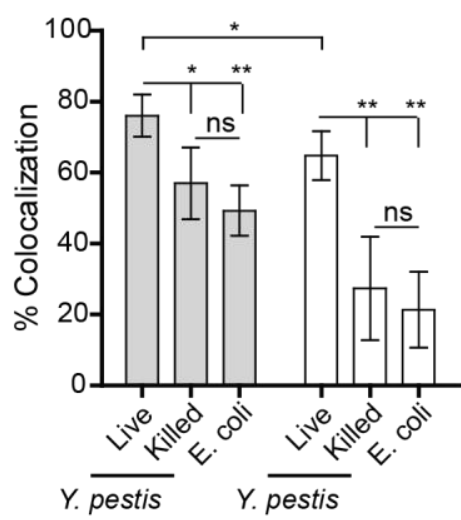

C

Rab11b

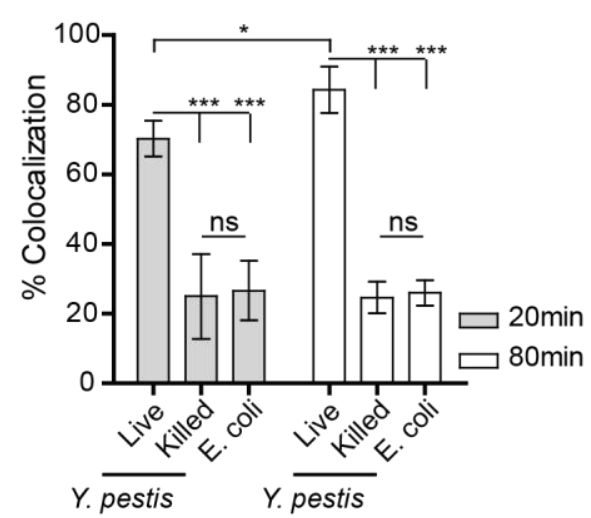

Figure 4-8. Y. pestis acquires recycling endosome markers Rab4a and Rab11b.

To determine if the bacteria recruited Rabs 4a and/or 11b, RAW 264.7 cells were transiently transfected with either pEGFP-Rab4a or pEGFP-Rab11b, and infected with either live or paraformaldehyde killed Y. pestis CO92 pCD1(-) mCherry (MOI 3) and E. coli mCherry (MOI 20) 24 h later.(A) Representative images at 20 min post-infection with cropped bacteria inserts. (B and C) Rab4a and Rab11b percent colocaliztion represented as mean \pm std of all biological repeats $(n=3)$. Significance calculated by one-way ANOVA or Student's T-test. ${ }^{*}=p V \leq 0.05 ;{ }^{* *}=p V \leq 0.01 ;{ }^{* * *}=$ $\mathrm{pV} \leq 0.001 ; \mathrm{ns}=$ not significant. Scale bar $10 \mu \mathrm{m}$ and insert $2 \mu \mathrm{m}$. 
A

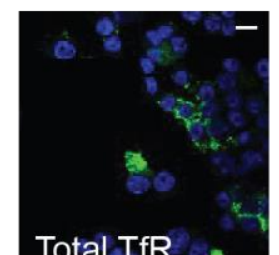

B
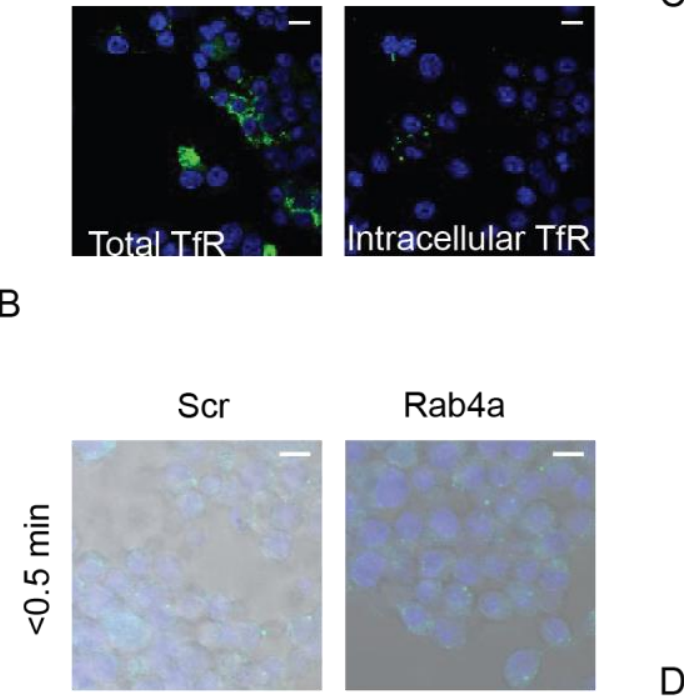
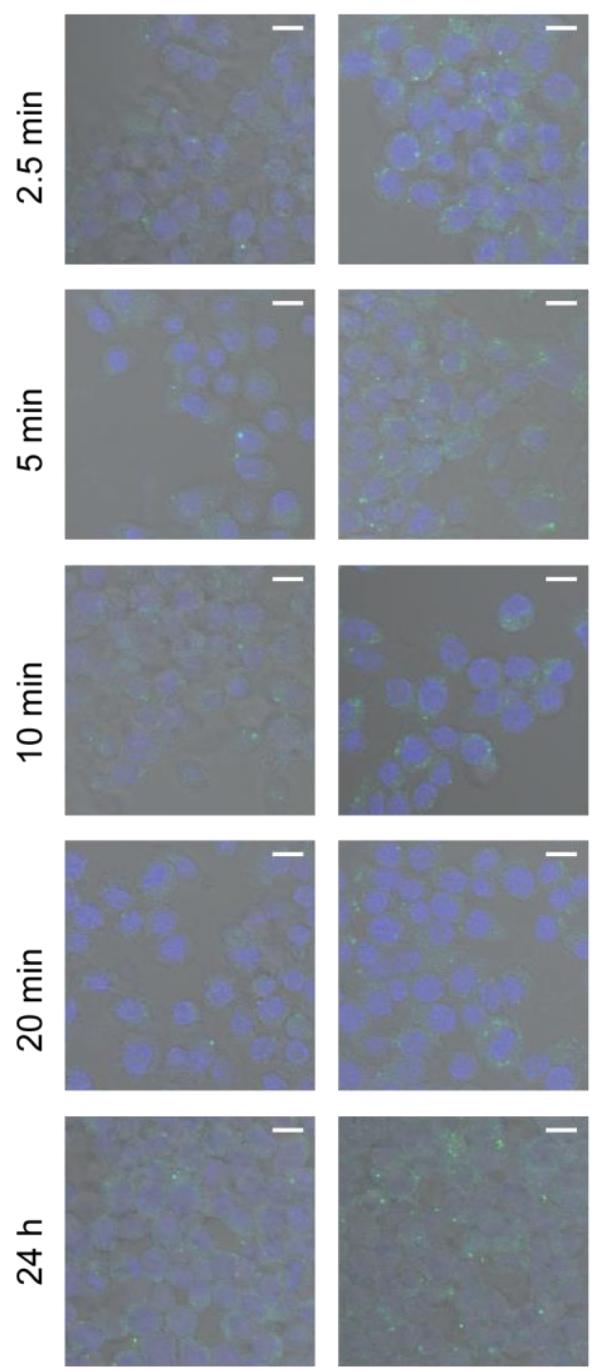

C Intensity per cell

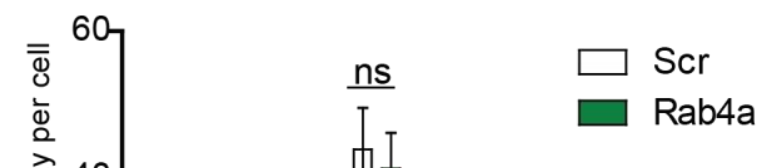

D

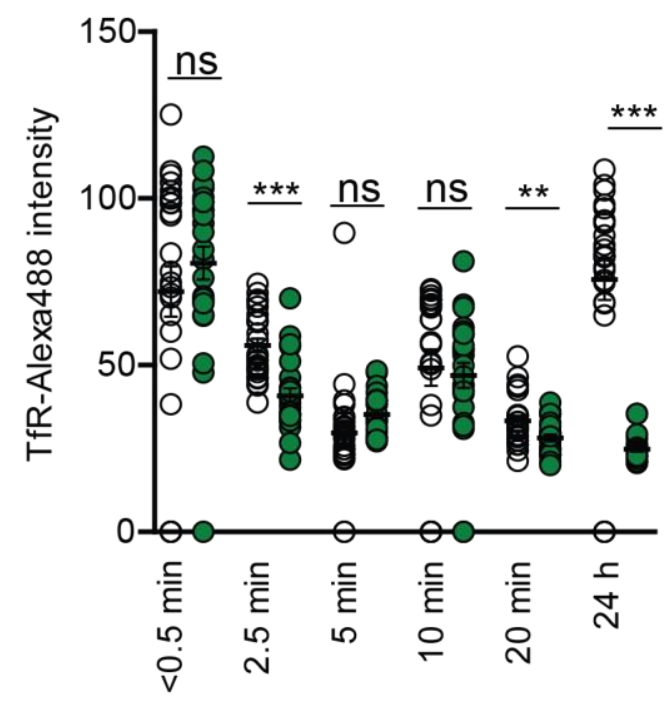

E

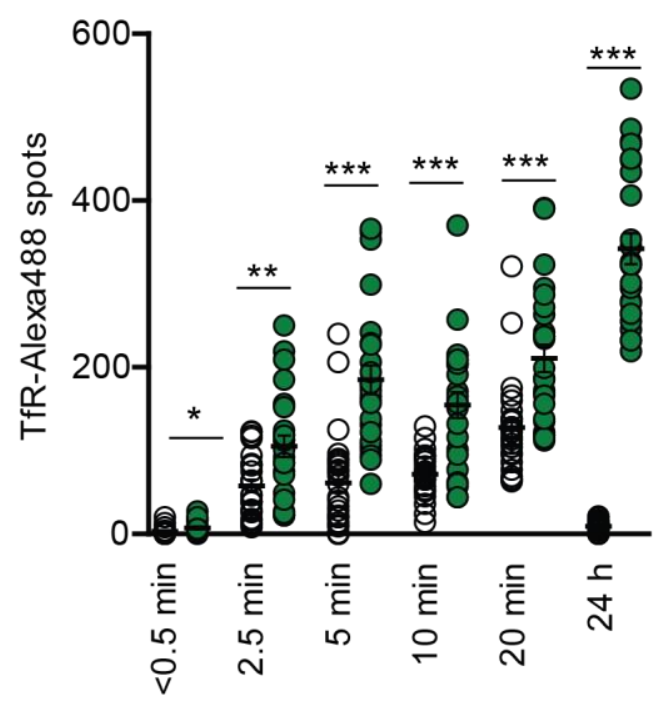


Figure 4-9. Recycling assay measures TfR kinetics.

RAW 264.7 cells were transfected with either scrambled (scr) or Rab4a siRNA for $48 \mathrm{~h}$ prior to synchronization, pulsing with anti-TfR antibody, fixed and counterstained with Alexa488 secondary antibody ( $n=25)$. (A) Representative images of total TfR staining vs only internalized TfR after acidic washing to remove surface and non-specifically bound antibody. (B) Representative images over the kinetic time course for either scr or Rab4a treated cells. (C) Internalized TfR Intensity per cell for either scr or Rab4a treated cells over the kinetic time course ((intensity of TfR $\times$ TfR spots)/ cell number). (D)Receptor amount (intensity of TfR) from raw intensity of internalized TfR in either scr or Rab4a. (E)Endosome amount (\# TfR spots) in either scr or Rab4a. Significance calculated by Student's T-test. Graphed as mean \pm SEM. ${ }^{*}=p V \leq 0.05^{* *}=p V \leq 0.01 ;{ }^{* *}=p V \leq 0.001$; $n s=$ not significant. Scale bar $10 \mu \mathrm{m}$. 
A 24 h post-infection

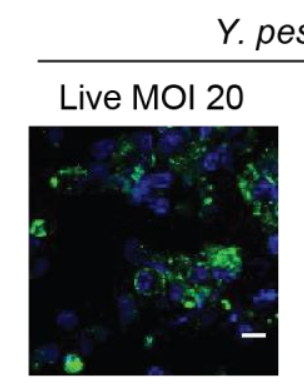

B

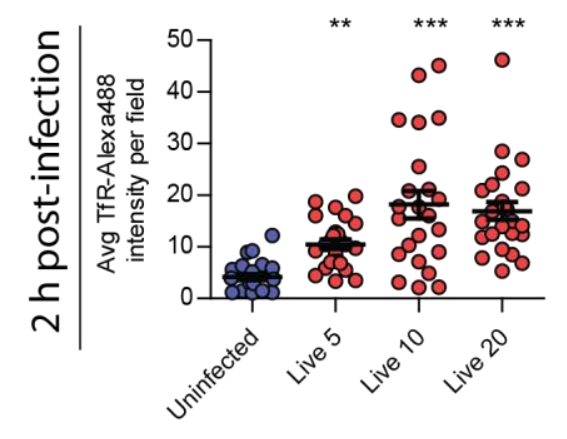

E

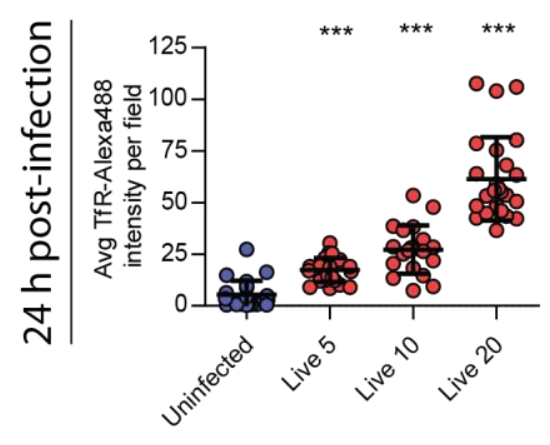

C

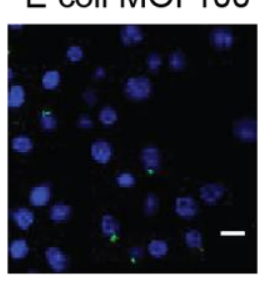

Uninfected

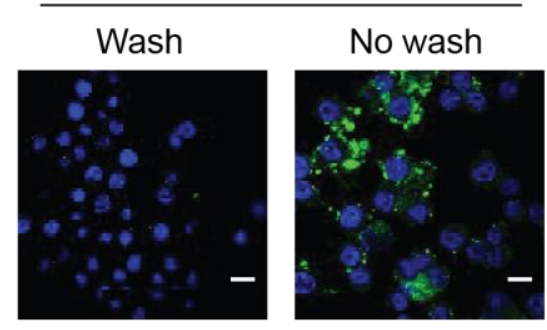

D

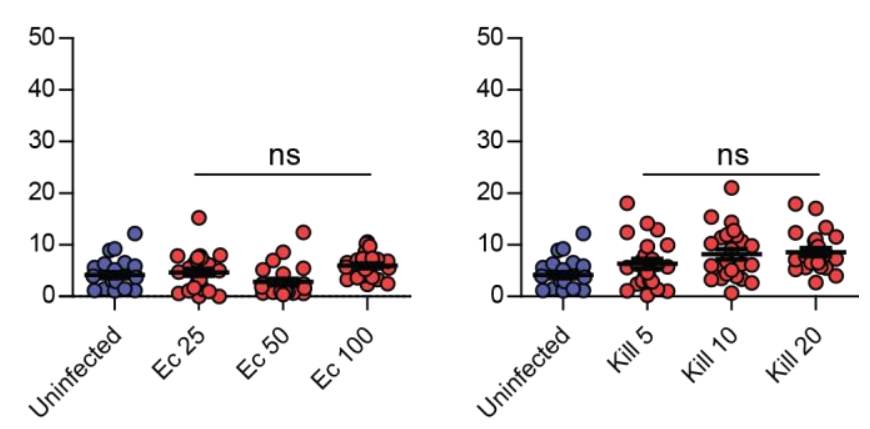

F

G
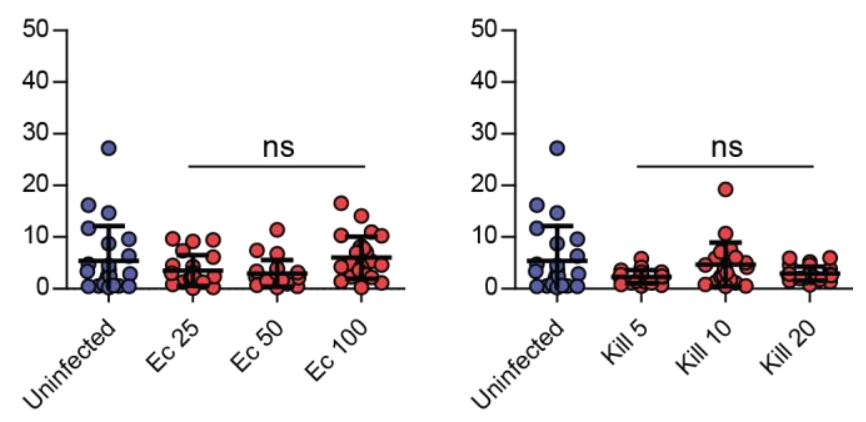

$\mathrm{H}$

HMDMs 2 h post-infection

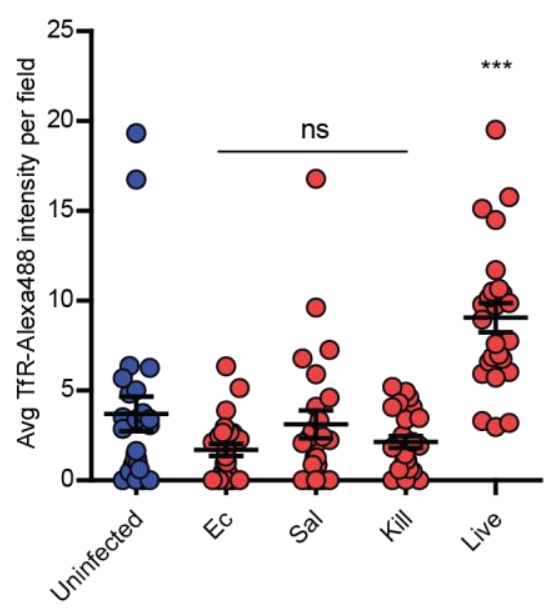

HMDMs 24 h post-infection

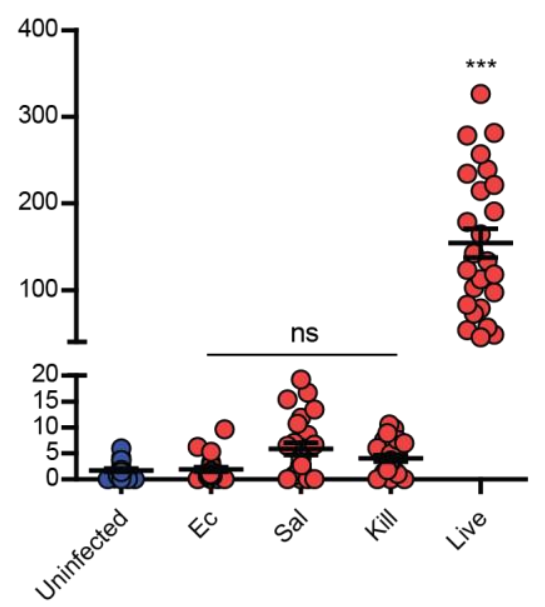


Figure 4-10. Y. pestis infection stalls host cell recycling.

(A)Representative images at $24 \mathrm{~h}$ post-infection in RAW264.7 cells. (B-G)Avg TfR intensity per field for infected RAW2647 cells with either dsRed live or paraformaldehyde killed Y. pestis (MOI 5, 10, 20) or E. coli (MOI 25, 50, 100). (H \& I)Avg TfR intensity per field for HMDMs infected with either dsRed live or paraformaldehyde killed Y. pestis (MOI 10), E. coli (100) or Salmonella (MOI 100). Significance calculated by one-way ANOVA using Dunnett's multiple comparison test to uninfected control. Graphed as a representative experiment with mean \pm SEM. ${ }^{* *}=p V \leq 0.01 ;{ }^{* * *}$ $=p V \leq 0.001 ; n s=$ not significant. Scale bar $10 \mu \mathrm{m}$. 


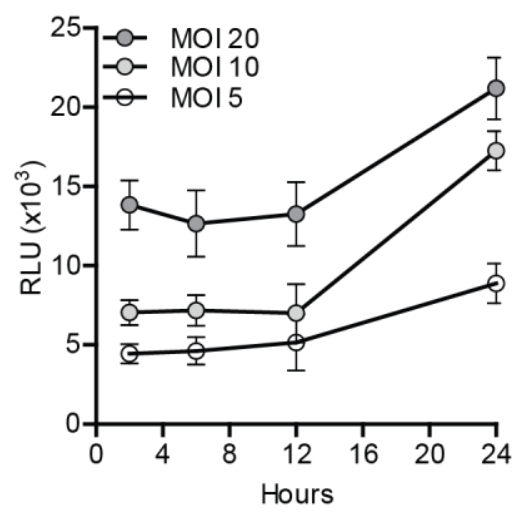

Figure 4-11. Ant-TfR antibody does not impact $Y$. pestis intracellular growth.

RAW264.7 cells were infected with Y. pestis CO92 pCD1(-)LuXPtolc $(n=8 ; M O I ~ 5,10,20)$ for 20 mins. Extracellular bacteria were killed, and intracellular bacteria maintained in gentamicin. Anti-TfR antibody was maintained in the media throughout the experiment. Bioluminescence was monitored at 2, 6, 12 and $24 \mathrm{~h}$ post-infection. 

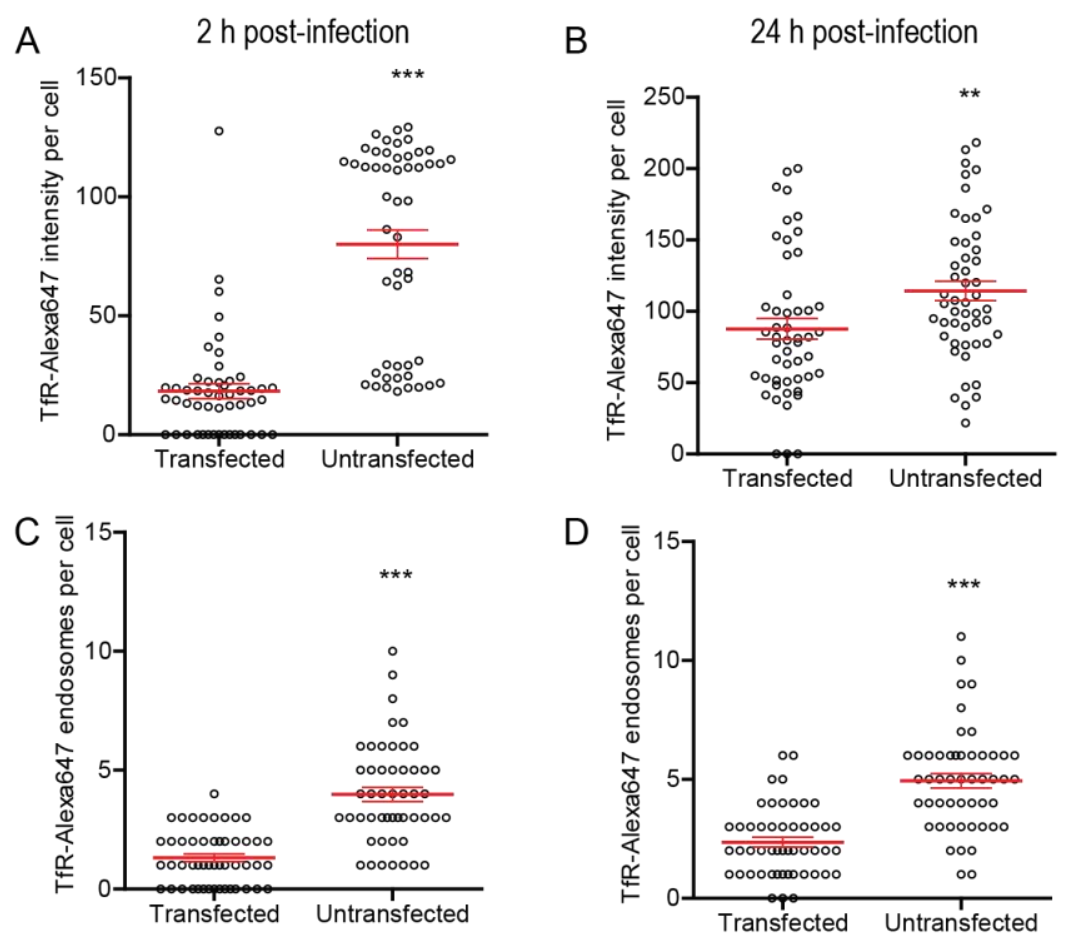

E
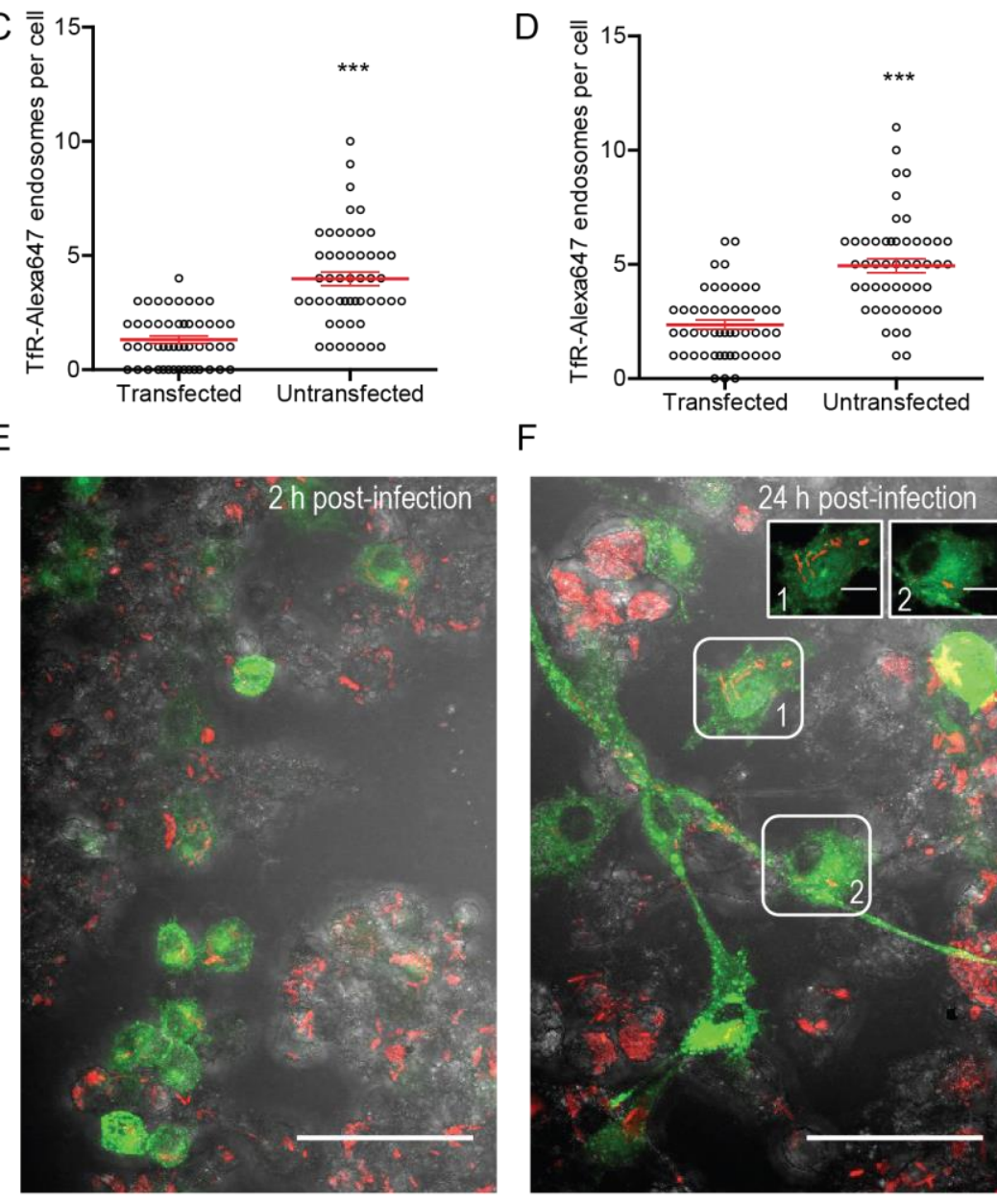

F
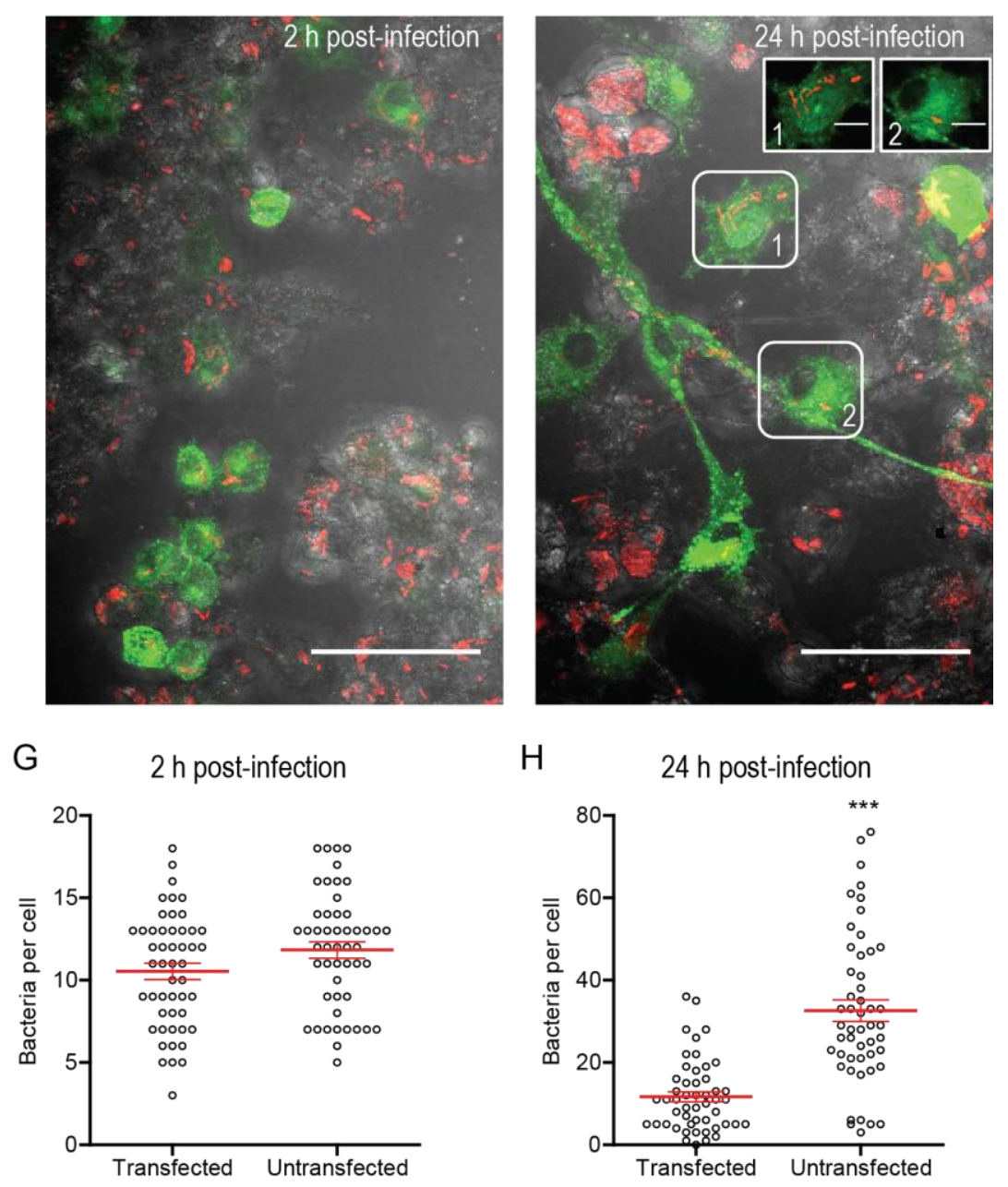
Figure 4-12. Stalling host cell recycling is required for $Y$. pestis replication.

RAW264.7 macrophages were transiently transfected with pEGFP-Rab11b prior to infection with Y. pestis CO92 pCD1(-) pGEN::mCherry (MOI 10) in a recycling assay. (A \& B) 2 h post-infection of TfR intensity and endosomes for transfected vs untransfected cells $(n=50)$. (C \& D) 24 h postinfection of TfR intensity and endosomes for transfected vs untransfected cells $(n=50)$. ( $E$ \& F) representative field images at 2 and $24 \mathrm{~h}$ post-infection. (G) $2 \mathrm{~h}$ post-infection bacteria per transfected vs untransfected cells $(n=50)$. (H) 24 h post-infection bacteria per transfected vs untransfected cells $(n=50)$. Significance calculated by paired Student's T-test. Graphed as a representative experiment with mean \pm SEM. ${ }^{* *}=p V \leq 0.01 ;{ }^{* * *}=p V \leq 0.001$; Scale bar field $50 \mu m$ and insert $10 \mu \mathrm{m}$. 
$\underline{\text { Tables }}$

Table 4-1. 135 validated genes

\begin{tabular}{|c|c|c|c|c|c|c|}
\hline \multicolumn{4}{|c|}{ siRNA Gene Target Identifers } & \multicolumn{3}{|c|}{10 Hour Normalized $Y$. pestis Survival for Genes Meeting Cutoffs } \\
\hline Entrez Gene ID & Gene Symbol & MGI Gene/Marker ID & Ensembl ID & CO92 Primary & CO92 Secondary & KIM-D-19 Secondary \\
\hline 12266 & C3 & MGI:88227 & ENSMUSG000000024164 & 0.31 & 0.45 & 0.73 \\
\hline 14064 & $\mathrm{~F} 2 \mathrm{rl2}$ & MGI:1298208 & ENSMUSG00000021675 & 0.27 & 0.89 & 0.60 \\
\hline 14635 & Galk1 & MGI:95730 & ENSMUSG000000020766 & 0.27 & 0.47 & 0.62 \\
\hline 14676 & Gna15 & MGI:95770 & ENSMUSG00000034792 & 0.28 & 0.51 & 0.76 \\
\hline 14967 & H2-D4 & MGI:95899 & & 0.32 & 0.73 & 0.60 \\
\hline 14997 & H2-M9 & MGI:1276570 & ENSMUSG00000067201 & 0.20 & 0.66 & 0.41 \\
\hline 15002 & $\mathrm{H} 2-\mathrm{Ob}$ & MGI:95925 & ENSMUSG00000041538 & 0.21 & 0.57 & 0.50 \\
\hline 15384 & Hnrnpab & MGI:1330294 & ENSMUSG000000020358 & 0.14 & 0.27 & 0.35 \\
\hline 15387 & Hnrnpk & MGI:99894 & ENSMUSG00000021546 & 0.19 & 0.30 & 0.56 \\
\hline 15482 & Hspa1l & MGI:96231 & ENSMUSG00000007033 & 0.34 & 0.21 & 0.22 \\
\hline 16174 & Il18rap & MGI:1338888 & ENSMUSG000000026068 & 0.29 & 0.36 & 0.40 \\
\hline 16678 & Krt1 & MGI:96698 & ENSMUSG00000046834 & 0.24 & 0.38 & 0.27 \\
\hline 16950 & Loxl3 & MGI:1337004 & ENSMUSG00000000693 & 0.26 & 0.55 & 0.46 \\
\hline 17135 & Mafk & MGI:99951 & ENSMUSG000000018143 & 0.20 & 0.82 & 0.41 \\
\hline 17195 & $\mathrm{Mbl2}$ & MGI:96924 & ENSMUSG000000024863 & 0.01 & 0.50 & 0.45 \\
\hline 17279 & Melk & MGI:106924 & ENSMUSG000000035683 & 0.27 & 0.47 & 0.24 \\
\hline 17289 & Mertk & MGI:96965 & ENSMUSG00000014361 & 0.28 & 0.43 & 0.50 \\
\hline 17385 & Mmp11 & MGI:97008 & ENSMUSG000000000901 & 0.12 & 0.95 & 0.49 \\
\hline 17527 & Mpv17 & MGI:97138 & ENSMUSG000000090262 & 0.35 & 0.60 & 0.53 \\
\hline 17540 & Mrvi1 & MGI:1338023 & ENSMUSG000000005611 & 0.20 & 0.67 & 0.43 \\
\hline 17896 & Myl4 & MGI:97267 & ENSMUSG000000061086 & 0.11 & 0.31 & 0.29 \\
\hline 17904 & Myl6 & MGI:109318 & ENSMUSG000000090841 & 0.16 & 0.59 & 0.40 \\
\hline 17907 & Mylpf & MGI:97273 & ENSMUSG00000030672 & 0.06 & 1.00 & 0.59 \\
\hline 17925 & Myo9b & MGI:106624 & ENSMUSG000000004677 & 0.09 & 0.58 & 0.58 \\
\hline 17932 & Myt1 & MGI:1100535 & ENSMUSG00000010505 & 0.35 & 0.56 & 0.25 \\
\hline 18007 & $\mathrm{Neo1}$ & MGI:1097159 & ENSMUSG000000032340 & 0.17 & 0.54 & 0.63 \\
\hline 18044 & Nfya & MGI:97316 & ENSMUSG000000023994 & 0.04 & 0.69 & 0.46 \\
\hline 18071 & Nhlh1 & MGI:98481 & ENSMUSG000000051251 & 0.09 & 0.54 & 0.45 \\
\hline 18082 & Nipsnap1 & MGI:1278344 & ENSMUSG00000034285 & 0.19 & 0.34 & 0.36 \\
\hline 18143 & Npas2 & MGI:109232 & ENSMUSG000000026077 & 0.20 & 0.37 & 0.46 \\
\hline 18181 & Nrf1 & MGI:1332235 & ENSMUSG000000058440 & 0.09 & 0.20 & 0.27 \\
\hline 18201 & Nsmaf & MGI:1341864 & ENSMUSG000000028245 & 0.16 & 0.57 & 0.35 \\
\hline 18209 & $\mathrm{Ntn} 3$ & MGI:1341188 & ENSMUSG00000079662 & 0.35 & 0.81 & 0.50 \\
\hline 18530 & Pcdh8 & MGI:1306800 & ENSMUSG000000036422 & 0.17 & 1.08 & 0.56 \\
\hline 18538 & Pcna & MGI:97503 & ENSMUSG000000027342 & 0.17 & 0.40 & 0.39 \\
\hline 18641 & Pfkl & MGI:97547 & ENSMUSG000000020277 & 0.15 & 0.57 & 0.52 \\
\hline 18690 & Phxr5 & MGI:104521 & & 0.20 & 0.55 & 0.38 \\
\hline 18720 & Pip5k1a & MGI:107929 & ENSMUSG000000028126 & 0.28 & 0.78 & 0.46 \\
\hline 18744 & Pja1 & MGI:1101765 & ENSMUSG000000034403 & 0.26 & 0.37 & 0.44 \\
\hline 18992 & Pou3f2 & MGI:101895 & ENSMUSG000000095139 & 0.20 & 0.90 & 0.49 \\
\hline 19053 & Ppp2cb & MGI:1321161 & ENSMUSG000000009630 & 0.15 & 0.72 & 0.51 \\
\hline 19064 & Ppy & MGI:97753 & ENSMUSG00000017316 & 0.23 & 1.16 & 0.57 \\
\hline 19110 & Prl4a1 & MGI:1206587 & ENSMUSG000000005891 & 0.33 & 0.27 & 0.19 \\
\hline 19331 & Rab19 & MGI:103292 & ENSMUSG000000029923 & 0.25 & 0.42 & 0.14 \\
\hline 19332 & Rab20 & MGI:102789 & ENSMUSG00000031504 & 0.23 & 0.53 & 0.37 \\
\hline 19335 & Rab23 & MGI:99833 & ENSMUSG000000004768 & 0.18 & 0.41 & 0.35 \\
\hline 19340 & Rab3d & MGI:97844 & ENSMUSG000000019066 & 0.27 & 0.73 & 0.59 \\
\hline 19341 & Rab4a & MGI:105069 & ENSMUSG000000019478 & 0.13 & 0.33 & 0.10 \\
\hline 20195 & S100a11 & MGI:1338798 & ENSMUSG000000027907 & 1.59 & 1.12 & 0.63 \\
\hline 20333 & $\operatorname{Sec} 22 b$ & MGI:1338759 & ENSMUSG000000027879 & 0.16 & 0.45 & 0.51 \\
\hline 20336 & Exoc4 & MGI:1096376 & ENSMUSG000000029763 & 1.50 & 1.16 & 0.87 \\
\hline 20438 & Siah1b & MGI:108063 & ENSMUSG000000040749 & 0.33 & 0.12 & 0.20 \\
\hline 20610 & Sumo3 & MGI:1336201 & ENSMUSG000000020265 & 0.32 & 0.50 & 0.47 \\
\hline 20630 & Snrpc & MGI:109489 & ENSMUSG000000024217 & 0.35 & 0.63 & 0.58 \\
\hline 21454 & Tcp1 & MGI:98535 & ENSMUSG00000068039 & 0.25 & 0.62 & 0.44 \\
\hline 21787 & Tfg & MGI:1338041 & ENSMUSG000000022757 & 0.34 & 0.38 & 0.51 \\
\hline 22110 & Tspyl1 & MGI:1298395 & ENSMUSG000000047514 & 0.32 & 0.55 & 0.50 \\
\hline 26394 & Lypla2 & MGI:1347000 & ENSMUSG000000028670 & 0.27 & 0.46 & 0.28 \\
\hline 27395 & Mrpl15 & MGI:1351639 & ENSMUSG000000033845 & 0.11 & 0.25 & 0.27 \\
\hline 54633 & Pqbp1 & MGI:1859638 & ENSMUSG000000031157 & 0.27 & 0.45 & 0.60 \\
\hline 56041 & Uso1 & MGI:1929095 & ENSMUSG000000029407 & 0.15 & 0.39 & 0.31 \\
\hline 56096 & Plac1 & MGI:1926287 & ENSMUSG000000061082 & 0.34 & 0.58 & 0.48 \\
\hline 56284 & Mrpl19 & MGI:1926274 & ENSMUSG000000030045 & 0.33 & 0.29 & 0.30 \\
\hline 56522 & Papolb & MGI:1932115 & ENSMUSG000000074817 & 0.26 & 0.60 & 0.66 \\
\hline 56523 & Pmfbp1 & MGI:1930136 & ENSMUSG000000031727 & 0.34 & 0.62 & 0.43 \\
\hline 58220 & Pard6b & MGI:2135605 & ENSMUSG000000044641 & 0.36 & 0.33 & 0.29 \\
\hline
\end{tabular}




\begin{tabular}{|c|c|c|c|c|c|c|}
\hline \multicolumn{4}{|c|}{ siRNA Gene Target Identifers } & \multicolumn{3}{|c|}{10 Hour Normalized $Y$. pestis Survival for Genes Meeting Cutoffs } \\
\hline Entrez Gene ID & Gene Symbol & MGI Gene/Marker ID & Ensembl ID & CO92 Primary & CO92 Secondary & KIM-D-19 Secondary \\
\hline 58234 & Shank3 & MGI:1930016 & ENSMUSG00000022623 & 0.34 & 0.31 & 0.46 \\
\hline 64384 & Sirt3 & MGI:1927665 & ENSMUSG00000025486 & 0.25 & 0.65 & 0.40 \\
\hline 66409 & RsI1d1 & MGI:1913659 & ENSMUSG00000005846 & 0.22 & 0.33 & 0.31 \\
\hline 66615 & Atg4b & MGI:1913865 & ENSMUSG00000026280 & 0.24 & 0.38 & 0.20 \\
\hline 66681 & Pgm1 & MGI:97564 & ENSMUSG00000029171 & 0.28 & 0.44 & 0.58 \\
\hline 67474 & Snap29 & MGI:1914724 & ENSMUSG00000022765 & 0.37 & 0.41 & 0.57 \\
\hline 67717 & Lipf & MGI:1914967 & ENSMUSG00000024768 & 0.26 & 0.50 & 0.46 \\
\hline 67832 & Brix1 & MGI:1915082 & ENSMUSG00000022247 & 0.10 & 0.35 & 0.28 \\
\hline 68572 & Ict1 & MGI:1915822 & ENSMUSG00000018858 & 0.20 & 0.83 & 0.52 \\
\hline 68943 & Pink1 & MGI:1916193 & ENSMUSG00000028756 & 0.20 & 0.74 & 0.39 \\
\hline 69902 & Mrto4 & MGI:1917152 & ENSMUSG00000028741 & 0.25 & 0.54 & 0.47 \\
\hline 70152 & Mett17a1 & MGI:1916523 & ENSMUSG00000054619 & 0.28 & 0.87 & 0.49 \\
\hline 71306 & Mfap3I & MGI:1918556 & ENSMUSG00000031647 & 0.24 & 1.16 & 0.46 \\
\hline 71472 & Usp19 & MGI:1918722 & ENSMUSG00000006676 & 1.46 & 1.33 & 0.68 \\
\hline 71740 & Pvrl4 & MGI:1918990 & ENSMUSG00000006411 & 0.21 & 0.40 & 0.26 \\
\hline 71883 & Coq2 & MGI:1919133 & ENSMUSG00000029319 & 0.28 & 0.31 & 0.43 \\
\hline 72183 & Snx6 & MGI:1919433 & ENSMUSG00000005656 & 0.36 & 0.56 & 0.67 \\
\hline 73419 & Armt1 & MGI:1920669 & ENSMUSG00000061759 & 0.14 & 0.55 & 0.41 \\
\hline 74053 & Grip1 & MGI:1921303 & ENSMUSG00000034813 & 0.35 & 0.28 & 0.21 \\
\hline 74116 & Pi16 & MGI:1921366 & ENSMUSG00000024011 & 0.33 & 0.15 & 0.31 \\
\hline 74143 & Opa1 & MGI:1921393 & ENSMUSG00000038084 & 0.36 & 0.32 & 0.20 \\
\hline 74782 & Glt8d2 & MGI:1922032 & ENSMUSG00000020251 & 0.27 & 0.57 & 0.56 \\
\hline 75292 & Prkd3 & MGI:1922542 & ENSMUSG00000024070 & 0.30 & 0.87 & 0.59 \\
\hline 75530 & Lyrm7 & MGI:1922780 & ENSMUSG00000020268 & 0.30 & 0.34 & 0.30 \\
\hline 75985 & Rab30 & MGI:1923235 & ENSMUSG00000030643 & 0.39 & 0.19 & 0.11 \\
\hline 76308 & Rab1b & MGI:1923558 & ENSMUSG00000024870 & 0.13 & 0.76 & 0.35 \\
\hline 76338 & Rab2b & MGI:1923588 & ENSMUSG00000022159 & 0.14 & 0.42 & 0.12 \\
\hline 76366 & Mtif3 & MGI:1923616 & ENSMUSG00000016510 & 0.16 & 0.21 & 0.12 \\
\hline 77604 & $\mathrm{Rbm} 12 \mathrm{~b} 2$ & MGI:1924854 & ENSMUSG00000052137 & 0.13 & 0.25 & 0.26 \\
\hline 81500 & Sil1 & MGI:1932040 & ENSMUSG00000024357 & 0.12 & 0.70 & 0.60 \\
\hline 83672 & Syt13 & MGI:1933367 & ENSMUSG00000041831 & 0.14 & 0.60 & 0.55 \\
\hline 98685 & Trmt1l & MGI:1916185 & ENSMUSG00000053286 & 1.88 & 1.46 & 0.57 \\
\hline 98758 & Hnrnpf & MGI:2138741 & ENSMUSG00000042079 & 0.16 & 0.67 & 0.53 \\
\hline 104776 & Aldh6a1 & MGI:1915077 & ENSMUSG00000021238 & 0.36 & 0.43 & 0.53 \\
\hline 108011 & Ap4e1 & MGI:1336993 & ENSMUSG00000001998 & 0.37 & 0.26 & 0.25 \\
\hline 108069 & Grm3 & MGI:1351340 & ENSMUSG00000003974 & 0.29 & 0.30 & 0.52 \\
\hline 108853 & Mtrf1I & MGI:1918830 & ENSMUSG00000019774 & 0.32 & 0.24 & 0.21 \\
\hline 109323 & C1qtnf7 & MGI:1925911 & ENSMUSG00000061535 & 0.17 & 0.40 & 0.43 \\
\hline 140859 & Nek8 & MGI:1890646 & ENSMUSG00000017405 & 0.28 & 0.34 & 0.72 \\
\hline 170719 & Oxr1 & MGI:2179326 & ENSMUSG00000022307 & 0.32 & 0.25 & 0.38 \\
\hline 192232 & Hps4 & MGI:2177742 & ENSMUSG00000042328 & 1.48 & 1.10 & 0.92 \\
\hline 207227 & Stxbp5I & MGI:2443815 & ENSMUSG00000022829 & 0.24 & 0.30 & 0.31 \\
\hline 207920 & Esrp1 & MGI:1917326 & ENSMUSG00000040728 & 0.17 & 0.44 & 0.28 \\
\hline 208177 & Phldb2 & MGI:2444981 & ENSMUSG00000033149 & 0.30 & 0.41 & 0.47 \\
\hline 209456 & Trp53bp2 & MGI:2138319 & ENSMUSG00000026510 & 0.30 & 0.82 & 0.55 \\
\hline 213556 & Plekhh2 & MGI:2146813 & ENSMUSG00000040852 & 0.32 & 0.30 & 0.46 \\
\hline 215335 & Slc36a1 & MGI:2445299 & ENSMUSG00000020261 & 0.33 & 0.73 & 0.51 \\
\hline 217371 & Rab40b & MGI:2183451 & ENSMUSG00000025170 & 0.31 & 0.55 & 0.32 \\
\hline 218121 & Mboat1 & MGI:2387184 & ENSMUSG00000038732 & 0.26 & 0.58 & 0.45 \\
\hline 218952 & Fermt2 & MGI:2385001 & ENSMUSG00000037712 & 0.28 & 0.51 & 0.27 \\
\hline 224044 & Cyp2ab1 & MGI:3644957 & ENSMUSG00000022818 & 0.36 & 0.62 & 0.35 \\
\hline 224118 & 0 & No associated gene & 0 & 0.37 & 0.57 & 0.13 \\
\hline 227157 & Mpp4 & MGI:2386681 & ENSMUSG00000079550 & 0.29 & 0.80 & 0.32 \\
\hline 228775 & Trib3 & MGI:1345675 & ENSMUSG00000032715 & 0.30 & 0.76 & 0.41 \\
\hline 229534 & Pbxip1 & MGI:2441670 & ENSMUSG00000042613 & 0.32 & 0.36 & 0.42 \\
\hline 231507 & Plac8 & MGI:2445289 & ENSMUSG00000029322 & 0.33 & 0.38 & 0.29 \\
\hline 233489 & Picalm & MGI:2385902 & ENSMUSG00000039361 & 1.59 & 1.25 & 1.12 \\
\hline 234852 & Chmp1a & MGI:1920159 & ENSMUSG00000000743 & 0.33 & 0.58 & 0.49 \\
\hline 235330 & Ttc12 & MGI:2444588 & ENSMUSG00000040219 & 0.09 & 0.53 & 0.37 \\
\hline 238831 & Ppwd1 & MGI:2443069 & ENSMUSG00000021713 & 0.30 & 0.56 & 0.51 \\
\hline 241035 & Pkhd1 & MGI:2155808 & ENSMUSG00000043760 & 0.33 & 0.30 & 0.49 \\
\hline 259100 & Olfr666 & MGI:3030500 & ENSMUSG00000063582 & 0.34 & 0.50 & 0.54 \\
\hline 268294 & Zbtb24 & MGI:3039618 & ENSMUSG00000019826 & 1.46 & 1.11 & 0.56 \\
\hline 269198 & Nbeal1 & MGI:2444343 & ENSMUSG00000073664 & 0.33 & 0.19 & 0.28 \\
\hline 279499 & Kctd19 & MGI:3045294 & ENSMUSG00000051648 & 0.06 & 0.69 & 0.33 \\
\hline 327814 & Ppfia2 & MGI:2443834 & ENSMUSG00000053825 & 0.27 & 0.65 & 0.47 \\
\hline 380795 & Ighg3 & MGI:2144790 & ENSMUSG00000076615 & 0.18 & 0.49 & 0.39 \\
\hline 381827 & 1700073E17Rik & MGI:1920734 & ENSMUSG00000087204 & 0.09 & 0.51 & 0.11 \\
\hline 403395 & Clec3a & MGI:2685642 & ENSMUSG00000008874 & 0.35 & 0.71 & 0.58 \\
\hline
\end{tabular}




\section{Table 4-2. Bacterial Strains}

Table 1. Bacterial Strains

Bacterial Strains

Y. pestis CO92 pCD1(-)

Y. pestis KIM D-19 pgm ${ }^{(-)} \mathrm{pCD}^{(+)}$

Y.pestisCO92 pCD1(-)pGEN- $P_{E M 7:: D s R E D}$

S. enterica Typhimurium

$S$. enterica Typhimurium pGEN- $P_{E M 7:: D s R E D}$

E. coli $\mathrm{DH} 5 \mathrm{a}$

E.coliK12 DH5apGEN-P $P_{E M 7:: D s R E D}$

E. coli DH5a pEGFP-Rab4a

E. coli DH5a pEGFP-Rab11b

References/sources

[9]

BEI Resources

[61]

ATCC 14028 (LT2)

LOU121; This study

New England Biolabs

[61]

[108]

[108] 
Table 4-3. siRNA

\begin{tabular}{ll}
\hline $\begin{array}{l}\text { Table 2. } \\
\text { siNRA }\end{array}$ & \\
\hline Target & Catalog \# \\
\hline Rab1b & 103033 \\
Rab4a & 64223 \\
Rab11b & 63944 \\
Myo5b & 63101 \\
\hline
\end{tabular}


Table 4-4. qRT-PCR Primers.

\begin{tabular}{llll}
$\begin{array}{lll}\text { Table } \mathbf{3} \\
\text { qRT-PC } \\
\text { Primers }\end{array}$ & & \\
\hline Target & $\mathbf{5}^{\prime}$ - Forward - 3' & \\
\hline Rab4a & CATCGTCCTTATCCTCTGCG & & AAAGCCTCTTCGACGTTCTC \\
Rab4b & CAGAAGTGGAAAGGAGCTGAG & & TCACCAGGAATTTGAAGAGGAAG \\
Rab5a & TGGTCAAGAACGGTATCATAGC & & GCCTTTGAAGTTCTTTAACCCAG \\
Rab7 & AATAGGAGCGGACTTTCTGAC & & CATCAAACACCAGAACACAGC \\
Rab9 & CACGGAAGATAGGTCAGAACAC & CCCTTTAATGCCATCAACAGC \\
Rab11a & GTGGGCAATAAGAGTGATTTACG & TCTGTTAGAATTGTCTGAAAAGCAG \\
Rab11b & AAGACCATCAAGGCTCAGATC & CGCTCCACGTTCTCATATGTC \\
Myo5b & ATTTGAGGAACGGGTCACAG & GGTCAGAGCAGATGGGTTATATG \\
GapDH & AATGGTGAAGGTCGGTGTG & ACAAGCTTCCCATTCTCGG \\
\hline
\end{tabular}


CHAPTER 5:

GENOME-WIDE RNAI HIGH-THROUGHPUT SCREEN IDENTIFIES

POTENTIAL GENES IMPACTING Y. PESTIS INVASION 
$\underline{\text { Introduction }}$

In order to establish an infection, bacteria must first adhere to, and then invade the host cell. Bacteria mediate adherence to host cells via adhesins that interact with host surface proteins to attach the bacteria to the plasma membrane $[260,261]$. In order to invade the host cell bacteria use either a trigger or zipper mechanism [260, 261]. The trigger mechanism is characterized by the activation of signaling cascades below the attached bacteria that "trigger" cytoskeletal rearrangements. These events cause plasma membrane ruffling around the organism, and ultimately lead to its internalization [260, 261]. This mechanism is well studied for Salmonella enterica Typhimurium [260, 261]. In this case, the bacteria uses the T3SS to inject effectors that trigger Rho GTPases that promote actin ruffling around the bacteria [260, 261]. The zipper mechanism occurs when bacterial proteins bind host membrane proteins creating an internalization signal ("outside-in") that induces the bacteria's uptake [260, 261]. An example of this is Invasin, a conserved molecule between $Y$. pseudotuberculosis and $Y$. enterocolitica that binds $\beta 1$ intergrins $[26,28,29,262,263]$. Through clustering of $\beta 1$ intergrins on the host cell surface the enteric Yersinia induce "outside-in" signaling and invade the host cell through the zipper mechanism [26].

We have observed that $Y$. pestis is rapidly taken up by the macrophage. Within 20 mins of a synchronized infection $>65 \%$ of total bacterial inoculum is intracellular. The speed and rate of invasion for $Y$. pestis is substantially higher than other well studied intracellular pathogens, such as $S$. enterica Typhimurium. During $S$. enterica Typhimurium infection of macrophages it takes nearly an hour, after synchronization, for roughly $\sim 50 \%$ of the inoculum to be intracellular (unpublished data) - an event that is a trigger mechanism mediated by SP1 island of the T3SS [264]. These observations lead us to hypothesize Y. pestis invasion is receptor mediated, through the zipper mechanism.

Y. pestis has several known adhesins, such as Ali, YadB, YadC, Pla and pH 6 antigen [1, 26, 265]. Studies of these adhesins in Y. pestis demonstrate their importance for adhering to host epithelial cells and professional phagocytes through interactions with host proteins, but clear links indicating their role in Y. pestis invasion has not been established [26, 265-267]. Furthermore, the 
Y. pestis T3SS is not used for invasion, and is actually well established to be anti-phagocytic [1]. Cowan et al. demonstrated that after a five min centrifugation to initially synchronize bacterial contact, $75 \%$ of $Y$. pestis inoculum can invade HeLa cells by 100 min post-infection [27]. These data confirm that $Y$. pestis is capable of invading epithelial cells just as efficiently as the enteric Yersinia species. Further, they showed that loss of pPCP (and specifically the Pla adhesin) had had a significant impact on invasion, but $\mathrm{Pla}(-)$ bacteria were still able to invade. Straley et al. demonstrated that $Y$. pestis lacking defined virulence determinants, such as the T3SS (encoded on pCD1 plasmid) and caf1 (capsule), are still capable of invading at high titers and survive within macrophages [54]. These studies highlight that an unknown chromosomally encoded effector contributes to bacterial invasion. Importantly, Straley and colleagues also show that opsinization of $Y$. pestis does not prevent bacterial invasion, nor does it significantly impact the ability of the bacteria to survive within these cells [54]. Together, these suggest an unknown interaction of a bacterial adhesin is responsible for $Y$. pestis invasion.

Since these observations, multiple attempts have been made to identify both the bacterial ligand and eukaryotic receptor used by $Y$. pestis for invasion [31, 49, 268-271]. Initially, $\mathrm{Ng}$ et al. conducted a microarray analysis of upregulated host genes during $Y$. pestis infection at $26^{\circ} \mathrm{C}$ in murine macrophages and identified Clec9a receptor [269]. However, $\mathrm{Ng}$ and colleagues were not specifically looking for receptors and neither this group nor others have followed up with this receptor. In 2004, two groups followed up on Ccr5 receptor mutation, termed CCR5 32 , which is a European conserved mutation linked to HIV resistance, and proposed to be a result of plague outbreak in the $14^{\text {th }}$ century $[268,271]$. In both studies, Ccr5 ${ }^{-/}$knockout mice had no significant difference in survival when challenged with Y. pestis [268, 271]. However, Elvin et al. demonstrate there was a significant difference in bacterial burden within infected Ccr5 ${ }^{-/}$macrophages [268]. These two studies concluded that $Y$. pestis entry and pathogenesis was more complex than previously thought.

Pla, and multifunctional protein encoded on the pPCP1 plasmid of Y. pestis, has been shown to bind to the macrophage receptor DEC-205 (also known as CD205) to promote 
dissemination [31]. However, these data failed to demonstrate clear evidence that preventing interactions with Pla were sufficient to perturb intracellular bacterial growth. Studies by Sodeinde at colleagues previously demonstrated the binding of Pla to CD205 found that Pla activity did not play a primary role in $Y$. pestis resistance to innate host defenses, but was important for dissemination of the bacteria, suggesting other factors contributed to $Y$. pestis invasion [177].

DC-SIGN (CD209) and Langrin (CD207) were demonstrated to bind LPS oligosaccharide core of $Y$. pestis [270, 272]. Y. pestis does not have an O-antigen, but an oligosaccharide outer core attached to lipid A (for review see [67]). In these studies KIM 10 pCD1 (-) strains were constructed with either a truncated outer core or O-polysaccharide covered core. Using these strains, in a fluorescence flow cytometry platform, they show the LPS core facilitates adherence to both dendritic and Langerhans cells, as mutants where the oligosaccharide core are truncated or covered by the O-polysaccharide are significantly attenuated [270, 273]. With the same strains they demonstrate lower intracellular bacterial burdens after killing extracellular bacteria for $1 \mathrm{~h}$ with gentamicin $(100 \mu \mathrm{g} / \mathrm{mL})[270,273]$. However, the data failed to provide a clear preventative role, as $Y$. pestis LPS is temperature modulated (hexa-acylated $\left(26^{\circ} \mathrm{C}\right)$ to tetra-acylated $\left(37^{\circ} \mathrm{C}\right)$; discussed in chapter 1). Furthermore, these studies failed to adequately quantify the relationship of bacterial inoculum, to either adherence or intracellular burden during in their flow cytometry assays.

While a few receptors have been identified through links with $Y$. pestis adhesins, the link to $Y$. pestis invasion is not well established in these works. The actual receptor(s) driving $Y$. pestis invasion of macrophages remains to be identified. During the optimization and completion of the Genome-wide RNAi HTS we emphasized our ability to kinetically monitor intracellular growth postinfection. We built our screening assay to have kinetic reads at $20 \mathrm{~min}, 2 \mathrm{~h}$ and $10 \mathrm{~h}$ post-infection with the idea that multiple time points might help us identify genes that were either a putative receptor, responsible for spacious YCV formation and bacterial replication, or differentially exploited during early and late YCV biogenesis. We noticed at 20 mins post-infection there was a consistent elevation in bioluminescence (RLU) in our Cop $\beta 1$ positive control over our scrambled 
(scr) negative control. This phenotype peaked our interest due to the consistency of our observations, and thus warranted further characterization. Here we describe the characterization of elevated 20 min RLU as an invasion phenotype from our genome-wide RNAi screen.

$\underline{\text { Results: }}$

Elevated RLU at 20 min post-infection indicates a defect in Y. pestis invasion.

During the genome-wide RNAi screen to identify host factors required for $Y$. pestis intracellular survival (Chapter 4) we noticed that Copß1 siRNA-treated wells had elevated bioluminescence ( 10-15\% higher) compared to scrambled (scr) negative controls across all screen plates ( $n=205)$ (Fig.5-1A). Copß1 is a cytosolic component of the cotamer complex implicated in non-clathrin dependent recycling of LDL receptors [274, 275]. This complex is also associated with ADP-ribosylation factors (ARFs) and Golgi vesicle trafficking [274, 275]. Knockdown of Cop $\beta 1$ has previously been shown to inhibit uptake of other pathogenic bacteria, such as L. monocytogenes [141]. Therefore, the elevated bioluminescence at 20 mins postinfection may indicate an invasion defect in Cop $\beta 1$ treated cells. Alternatively, it is possible that the phenotype was an artifact of the promoter driving our bioreporter ( $p T o / C)$. To test if elevated RLU was promoter dependent, we compared macrophage infection with Y.pestis CO92 pCD1(-)LuXPtolC to Y.pestis CO92 pCD1(-)LuXPCysz. These two promoters drive the same bacterial luciferase system and have been integrated into the chromosome of $Y$. pestis CO92. Both of the promoters have been characterized previously, and have direct correlation between bioluminescence and bacterial number (see chapter 2) [151]. Bioluminescence was read at 20 mins post-infection, Our results show both promoters have $~ 10-15 \%$ elevated bioluminescence at 20 mins post-infection in Cop $\beta 1$ treated cells in comparison to scrambled negative control (scr) (Fig. 5-1B). This data shows elevated RLU at 20 mins post-infection is independent of the promoter driving the bacterial luciferase system in Y. pestis CO92.

Since the phenotype was not promoter dependent, we next tested whether known inhibitors of $Y$. pestis invasion displayed the same phenotype. Previous studies with $Y$. pestis showed treatment with Cytochalasin D significantly impacts invasion of HeLa cells [27]. This chemical 
inhibitor has been demonstrated to prevent a range of cytoskeletal remodeling events to prevent phagocytosis [276]. Cytochalasin D has been extensively used to study bacterial invasion [253, 276-286]. Importantly, while Cytochalasin D treatment inhibits invasion, it has not been shown to not alter subsequent intracellular survival of $Y$. pestis. Therefore, treatment with Cytochalasin $D$ was chosen as a control to determine if inhibition of $Y$. pestis invasion of macrophages recapitulated the 20 min phenotype observed in Cop $\beta 1$ siRNA treated cells. Cytochalasin D treated cells were infected with Y. pestis CO92 pCD1(-)LuXPtolC for 20 min and bioluminescence was determined and compared to intracellular bacterial burden at 80 min post-infection. A dose dependent elevation in bacterial bioluminescence over our untreated control was observed in the Cytochalasin treated cells (Fig. 5-2A). This elevation correlated to a dose dependent decrease in the RLU of intracellular bacterial burden at 80 mins post-infection (Fig. 5-2B). These data confirm that elevated RLU at 20 mins post-infection is an indicator of invasion defect.

\section{Identifying invasion dataset from the Genome-wide RNAi HTS}

Having demonstrating that elevated bioluminescence at 20 mins post-infection is an indicator of an invasion defect, we sought to utilize this phenomena to identify potential invasion defects from the genome-wide RNAi screen. 20 min bioluminescence was normalized for our entire 20 min dataset $(n=16,757)$ by the average percent of the scrambled (scr) negative control on each individual plate. We established a cutoff criteria (materials and methods) and applied this to the entire 20 min dataset. This resulted in the identification of 1,075 genes with an invasion defect based on their elevated RLU at 20 mins post-infection (Table 5-1). To identify potential receptor/receptor activity of these hits we used PANTHER GO ontology database $[258,287]$. Of the 1,075 genes identified with an invasion defect, 96 (9\%) had direct receptor related functionalities (Table 5-2).

\section{Discussion}

Here we demonstrate that elevated bioluminescence (RLU) at 20 mins post-infection is an indication of a defect in Y. pestis invasion defects. Through our characterization of this phenotype, we show elevated RLU at 20 mins is: 1) independent of the promoter driving $Y$. pestis 
chromosomally integrated bioluminescent bioreporter, 2) using Cytochalasin D, a known invasion inhibitor, we are able to recapitulate a dose dependent elevation in 20 min RLU that is inversely proportional to the bacterial burden at 80 mins, based on intracellular RLU, and 3) our observations mimic previous data of Cowan et al., demonstrating that Cytochalasin D was capable of inhibiting Y. pestis invasion in HeLa cells [27]. Furthermore, from our phenotypic characterization we were able to identify a subset of genes from our genome-wide RNAi HTS as impacting Y. pestis invasion. This represents the first ever comprehensive list of potential receptors and invasion related components exploited by $Y$. pestis for entry into the host macrophage. Many of the invasion hits that are not classified as receptors, are actually signaling/ trafficking molecules downstream of these receptors, such as the Arfgaps (2, 15, 17, \& 18). Interestingly, Arfgap2 interacts with Arf1 to facilitate endocytosis and transport of endocytic cargo to the Golgi-ERGIC space [288]. Arf1 in turn is involved with Rab4a mediated endocytic recycling of receptors [238]. Of note, is the inclusion of Edg1 (Sphingosine 1-phosphate receptor 1) in our identified dataset. This receptor was shown by St. John and colleagues to be required by $Y$. pestis for efficient trafficking through the host lymphatics, and possibly entry into the macrophage (discussed below) [49] (Table 5-2). Moreover, none of the receptors identified impacting adherence of $Y$. pestis to the host cell (DEC 205, CD205, CD207 and CD209) were identified in our invasion dataset. This strongly suggests that while these receptors might contribute to adherence, they do not appear to contribute to $Y$. pestis invasion.

St. John et al. recently identified a requirement of S1P for dissemination from the draining lymph node during a bubonic infection model [49]. Their studies employed the use of chemical and antibody receptor agonists to block the trafficking of $Y$. pestis in the host lymphatics [49]. In their hands, Y. pestis was still partially able to colonize the host even without S1P. Furthermore, they demonstrated that from the site of infection there are two waves of infected cells that traffic to the draining lymph node. The first wave is double positive CD11c and CD11b cells, then over the course of 48 hrs the trafficked cells shift to CD11c, CD11b or PMN single positive cell types, which are representative on non-APC and inducible monocyte populations, respectively [49]. This novel observation highlighted that $Y$. pestis pathogenesis is potentially established through multiple innate immune cell types displaying a common receptor that facilitates $Y$. pestis adherence and 
invasion. This suggests that potentially the eukaryotic receptor exploited for $Y$. pestis invasion is also the receptor used to drive lymphatic dissemination.

The elevated RLU observed at 20 min post infection could be a result of : 1) Unimpeded light from extracellular bacteria results in the full amount of photons easily reaching the detector. Whereas, the light from intracellular bacteria has to escape both the YCV and the macrophage before reaching the detector, thusly dampening the amount of photons that are read. 2) Internalized bacteria undergo a rapid metabolic change that dampens light production due to substrate and ATP requirements of the luciferase operon. Whereas, extracellular bacteria do not shift their metabolic state. 3) After uptake of the bacteria within the macrophage bacteria that are being actively degraded do not produce light, resulting in decreased intracellular RLU in comparison to their extracellular counterparts. Regardless of the molecular mechanism driving the elevation in RLU from the luciferase system, we demonstrate that this is a strong indicator of a bacterial invasion defect.

There are three possible phenotypic outcomes post-invasion of the bacteria into the macrophage: 1) Elevated RLU is a reflection of strictly an invasion defect at 20 mins post-infection, and intracellular RLU at $80 \mathrm{~min} / 10 \mathrm{~h}$ is survival 2) Elevated RLU is an invasion defect, and loss of this invasion route forces the bacteria down a phagolysosome maturation pathway. Resulting in kinetically decreasing RLU at both 80 min and $10 \mathrm{~h}$ post-infection in comparison to scr control, and 3) Invasion of $Y$. pestis is not completely blocked by knockdown of the receptor, and a subpopulation of bacteria invade the macrophage via the normal mechanism resulting in decreased RLU at 80 min, but survival or growth (same RLU or increased RLU) at $10 \mathrm{~h}$. Either case, elevated 20 min RLU phenotypically indicates an invasion defect. However, we are currently characterizing the relationship between intracellular $\mathrm{RLU}$ at $80 \mathrm{~min}$ and $10 \mathrm{~h}$ post-infection to determine if these post invasion phenotypes reflect a branch point in $Y$. pestis survival due to disruption of the natural invasion route.

Cytochalasin $\mathrm{D}$ is an example of a true invasion defect in which there is an elevated RLU read at 20 mins followed by a decrease in RLU at $2 \mathrm{~h}$ but either remains stable or increases at the 
$10 \mathrm{~h} \mathrm{RLU}$ read (unpublished data). Rab1b is an example of RLU data showing no invasion defect, but a survival defect as RLU continues to decrease at $80 \mathrm{~min}$ and $10 \mathrm{~h}$ post-infection. We demonstrate that siRNA inhibition of Rab1b has no impact on bacterial invasion as the 20 min RLU is comparable to the scr negative control (unpublished data). Using confocal microscopy we demonstrate that $20-25 \%$ of the bacteria are extracellular, but the $80 \mathrm{~min}$ and $10 \mathrm{~h}$ data show decreasing RLU (chapter 4; [61]). We further show that the RLU decrease is a direct result of increase YCV acidification within 80 mins post-infection [61]. In contrast, RNAi of Cop $\beta 1$ is a primary example of both invasion and survival defect. There is an elevated 20 min RLU that correlates with confocal analysis showing $30-40 \%$ of the bacteria remain extracellular (chapter 4 ). However, at 80 min and $10 \mathrm{~h}$ the intracellular RLU decreases as a direct result of increased acidification of the YCV at 80 mins post-infection (unpublished data). From our data with Cytochalasin D, Rab1b and Copß1 there are three possible explanations for intracellular RLU at 80 mins/ $10 \mathrm{~h}$ with an elevated RLU at 20 mins for the $Y$. pestis invasion dataset. In order to test each outcome we would use comparisons of elevated RLU to YCV acidification.

The first outcome is that elevated RLU at 20 min strictly reflects invasion, and intracellular $\mathrm{RLU}$ at $80 \mathrm{~min}$ (or longer) is a measure of survival post-invasion. To test this hypothesis, we would need to test for acidification of the YCV at 80 mins post-infection for targets that have decreased RLU over time. Then, the results of acidification tests would need to be compared to RLU reads at 20 and 80 mins for a control that forces the bacteria toward the phagolysosome and ultimately degradation. In this instance, one would expect elevated RLU reads at 20 mins to have no correlation with increased YCV acidification or with increased intracellular RLU across a kinetic infection. Furthermore, determining this branching point between invasion and survival at 80 mins would implicate that the mechanism of bacterial entry is directly linked to avoidance of the phagolysosome, and is independent of the dogma that bacteria actively arrest phagolysosome maturation for survival.

The second possibility, is that blocking the natural entry mechanism of $Y$. pestis forces the bacteria toward a phagolysosome maturation pathway and results in bacterial death by 80 mins/ 
$10 \mathrm{~h}$. To determine this, a comparison of acidified YCVs of the target to scrambled negative control siRNA would need to be completed. One would expect to see a significant increase in acidified YCVs by 80 mins post-infection if elevated RLU at 20 mins is related to bacterial survival at 80 mins. Additionally, one would expect a continuous decrease in bacterial survival by RLU over time.

The third outcome that RNAi inhibition incompletely blocks $Y$. pestis invasion, and a subpopulation enters normally. This is based outcome is based on the caveats of RNAi. Firstly, RNAi penetrance is not $100 \%$ and the $80 \mathrm{~min} / 10 \mathrm{~h} \mathrm{RLU}$ is reflective of normal Y. pestis entry into macrophage cells that did not receive adequate knockdown of the specified host target. Penetrance meaning that one cannot determine expression within a single cell or the amount of siRNA that is distributed among cells. In this case further analysis of these genes would indicate that over time the bacteria that invade through the normal entry mechanism are able to survive and replicate. To address this hypothesis, we would need to test for acidification of the YCV at 80 mins post-infection. If there is no significant difference between YCV acidification of the target gene and the scrambled negative control, the elevated $20 \mathrm{~min} R L U$ in respect to decreased, non-acidified 80 min RLU would indicate an invasion defect, but would not indicate if $Y$. pestis invasion mechanism was directly linked to survival.

Overall, demonstrating the elevated RLU at 20 mins post-infection is a measure of $Y$. pestis invasion efficiency provides a powerful tool to dissect bacterial invasion and potentially identify the receptor of $Y$. pestis entry. While we have begun characterizing the various phenotypic results, additional studies are needed to determine the relationship of invasion to survival. A critical experiment for these studies is deciphering the RLU readouts of opsonized $Y$. pestis during macrophage infection, and if $Y$. pestis anti-sera prevents uptake. In regards to opsonization, one would expect to drive $Y$. pestis uptake via the Fc receptor and have no impact on bacterial survival as previously shown [54]. In contrast, using $Y$. pestis anti-sera at $26^{\circ} \mathrm{C}$, or live bacteria in a dose dependent response should provide an invasion phenotype or force the bacteria down a phagolysosome maturation pathway. We believe the latter is true, as previous studies elude to a Y. pestis specific invasion mechanism that is dependent on a chromosomally encoded protein [27]. 
That being said, if one saturates the normal binding receptor and forces the bacteria to enter via another mechanism it would shuttled to the phagolysosome and degraded in an acidified vacuole. Cowan et al., data supports the anti-sera hypothesis as entry of $Y$. pestis was attenuated during $26^{\circ} \mathrm{C}$ and $37^{\circ} \mathrm{C}$ temperature shifts due to an unknown chromosomally encoded bacterial protein, however, their data suggests it is due to phagolysosome trafficking [27].

\section{Materials and Methods}

Bacterial strains, siRNA transfection of macrophages.

Y. pestis CO92 [9] pCD1 ${ }^{(-)}$and derivatives (BEI Resources NR-4681) were cultivated at $26^{\circ} \mathrm{C}$ in Brain Heart Infusion (BHI) broth (Difco). Bioluminescent derivatives were generated using the LuXPtolc or LuXPCysz bioreporter as described previously [151]. RAW264.7 macrophages were obtained from ATCC and cultured in DMEM, $100 \mathrm{mM}$ glucose $+10 \%$ FBS (Hyclone). For siRNA transfection, $20 \mu \mathrm{l}$ of $0.165 \mu \mathrm{M}$ Silencer siRNA (Life Technologies) diluted in Opti-MEM (Life Technologies) was mixed with $10 \mu \mathrm{l}$ of $0.03 \%$ (v/v) Lipofectamine RNAi-Max/Opti-MEM (Life Technologies) as described by the manufacturer. $30 \mu$ of the siRNA-Lipofectamine complex was added to each well of a white flat-bottom 96-well plate (Greiner), incubated at room temperature for $10 \mathrm{~min}$, and then 1x104 RAW264.7 macrophages suspended in $80 \mu \mathrm{l}$ of DMEM+10\% FBS were added. Cells were incubated for $48 \mathrm{~h}$ at $37^{\circ} \mathrm{C}$ with $5 \% \mathrm{CO} 2$.

\section{$\underline{\text { Cytochalasin D treatment }}$}

Cytochalasin D was two-fold serially diluted from 2mM DMSO stock into DMSO and then diluted to final concentrations of $0.5,1$ and $2 \mu \mathrm{m}$ in DMEM 10\% FBS (Hyclone). The DMSO matched control $(0 \mu \mathrm{m})$ was used for analysis. Inhibitor and RAW264.7 murine macrophage cells were incubated for $1 \mathrm{hr}$ at $37^{\circ} \mathrm{C}$ with $5 \% \mathrm{CO}_{2}$ prior to bacterial infection. Final concentration of DMSO in DMEM 10\% FBS was $<3 \%$.

\section{Bacterial infection of macrophages.}

Macrophages were infected with Y. pestisCO92 pCD1(-)LuXPtolc or Y.pestis CO92 pCD1(LuXPCysz (MOI 10) as previously described [59, 61, 151]. In brief, bacteria cultivated overnight at 
$26^{\circ} \mathrm{C}$ in $\mathrm{BHI}$, washed in PBS, and diluted in prewarmed DMEM+10\%FBS. Bacteria were added to macrophages and infection synchronized by a $200 \times \mathrm{xg}$ centrifugation for 5 mins. 20 mins post infection extracellular bacteria were killed with gentamicin $(16 \mu \mathrm{g} / \mathrm{mL})$ for one hour, and then media was replaced with DMEM $+10 \%$ FBS containing $2 \mu \mathrm{g} / \mathrm{mL}$ gentamicin for the duration of the experiment. Bioluminescence was quantified using a Synergy HT or Synergy 4 plate reader (Biotek; $1 \mathrm{sec}$ read with sensitivity set at 150 ) at $20 \mathrm{~min}, 80 \mathrm{~min}$ and $10 \mathrm{~h}$ post-infection [151].

\section{Bioinformatics Analysis}

Using the Genome-wide RNAi HTS dataset, we first normalized the 20 min dataset to the RLU of the avg scr negative control per plate as follows: (x gene RLU / avg scr RLU). We then constructed a 95\% confidence interval $(\mathrm{Cl})$ around the \% elevation seen in the Cop $\beta 1$ controls per plate. We used the lower 95\% Cl limit as our cutoff criteria for \% elevated RLU to identify genes with an invasion defect per plate. For GO ontology analysis, Entrez Gene identifiers for all 1,075 genes meeting the lower $95 \% \mathrm{Cl}$ limit of Cop $\beta 1$ controls per plate were ran through PATNHER (http://www.pantherdb.org/) for their molecular processes based on GO ontology. Those Entrez Gene identifiers that were sub-classified under Receptor Activity (96) were used to generate a separate dataset.

\section{$\underline{\text { Statistics }}$}

All experiments were repeated in triplicate, and are displayed as single representative experiments. All statistics were calculated in Graph Pad using either Student's T-Test, or one way ANOVA with post-hoc Dunnett's multiple comparison test for significance. 
Figures and Figure Legends

A 20 min RLU from Genome-wide RNAi HTS

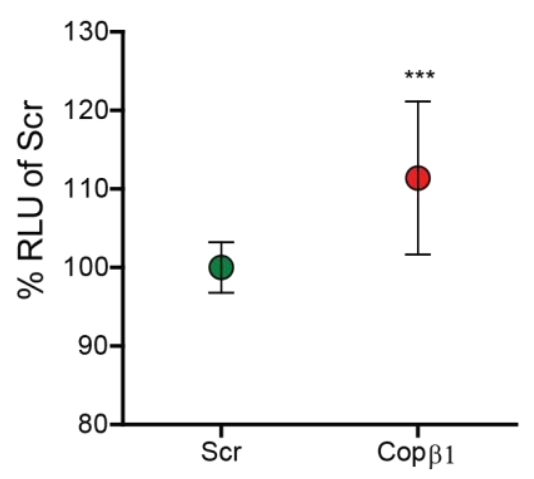

B 20 min post-infection

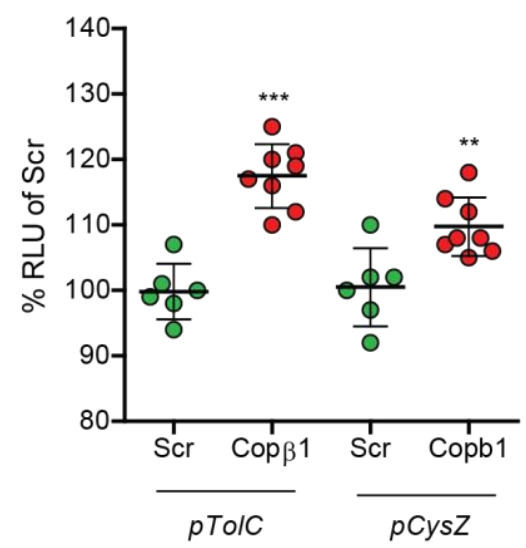

Figure 5-1. Elevated RLU is consistent and promoter independent.

(A) Bioluminescence (RLU) at 20 min post-infection for all genome-wide RNAi HTS plates ( $n=205)$.

(B) RAW264.7 macrophage cells transfected with either scrambled (scr; $n=6$ ) negative control or Copß1 $(n=8)$ siRNA for 48 hrs prior to infection with either Y.pestis CO92 pCD1(-)LuXPtolc or Y.pestis CO92 pCD1(-)LuXPCysz (MOI 10). Percent RLU compared to scr at 20 min. RLU = Relative Light Units. Significance calculated with Student's T-test; ${ }^{* *}=p<0.01,{ }^{* *}=p<0.001$. 
A 20 min post-infection

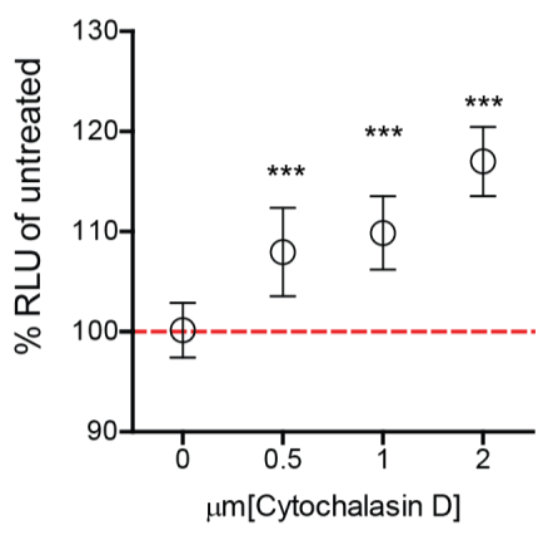

B 80 min post-infection

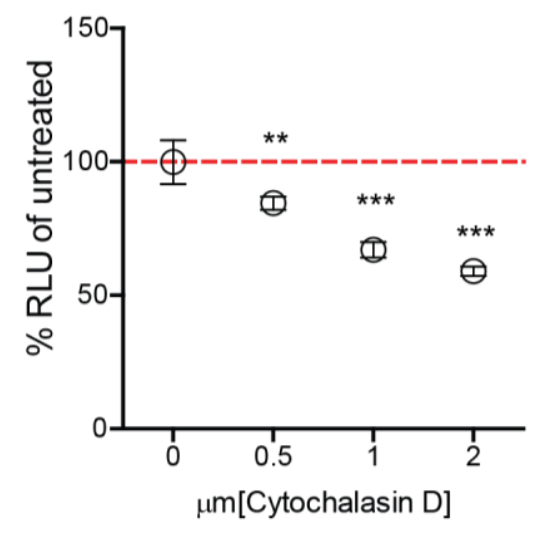

Figure 5-2. Elevated RLU is an invasion defect.

RAW264.7 macrophage cells treated with 0.5 , 1 , or $2 \mu$ m final [Cytochalasin D] $(n=6)$ for 1 hr prior to infection with Y.pestis CO92 pCD1(-)LuXPtolC (MOI 10). Bioluminescence (RLU) reads at (A) 20 and (B) 80 mins post-infection. Graphed as percent RLU compared to $0 \mu \mathrm{m}$ matched DMSO control. Dotted line $=100 \%$. RLU $=$ Relative Light Units. Significance calculated with One way ANOVA post-hoc Dunnett's multiple comparison test to $0 \mu \mathrm{m}$ matched DMSO control; ${ }^{* *}=p<0.01$, ${ }^{* * *}=p<0.001$. 
$\underline{\text { Tables }}$

Table 5-1. All putative invasion targets.

\begin{tabular}{|c|c|c|c|c|}
\hline \multirow{2}{*}{ Entrez Gene ID } & \multirow{2}{*}{ Gene Symbol } & \multicolumn{3}{|c|}{ Screen Survival } \\
\hline & & $20 \mathrm{~min}$ & $2.0 \mathrm{hr}$ & $10.0 \mathrm{hr}$ \\
\hline 13858 & Eps15 & 1.08 & 0.75 & 0.78 \\
\hline 13859 & Eps15-rs & 1.09 & 0.62 & 0.73 \\
\hline 14163 & Fgd1 & 1.09 & 0.72 & 0.78 \\
\hline 14469 & Gbp2 & 1.21 & 0.90 & 1.09 \\
\hline 14704 & Gng3 & 1.13 & 0.86 & 0.94 \\
\hline 14709 & Gng8 & 1.10 & 0.81 & 1.00 \\
\hline 330914 & Grit & 1.16 & 0.92 & 0.94 \\
\hline 69993 & Chn2 & 1.12 & 0.62 & 0.89 \\
\hline 78618 & Acap2 & 1.12 & 0.81 & 0.83 \\
\hline 11539.00 & Adora1 & 1.17 & 0.74 & 0.74 \\
\hline 67333 & Stk35 & 1.15 & 0.76 & 0.92 \\
\hline 68556 & Uckl1 & 1.21 & 0.79 & 0.92 \\
\hline 14744 & Gpr65 & 1.12 & 0.84 & 0.90 \\
\hline 15551 & $\mathrm{Htr} 1 \mathrm{~b}$ & 1.12 & 0.94 & 1.14 \\
\hline 16995 & Ltb4r1 & 1.10 & 0.88 & 1.02 \\
\hline 216749 & Nmur2 & 1.10 & 0.84 & 0.85 \\
\hline 227288 & Il8ra & 1.10 & 0.77 & 0.90 \\
\hline 387285 & Hcrtr2 & 1.15 & 0.92 & 1.00 \\
\hline 436440 & Gpr31c & 1.14 & 0.79 & 0.83 \\
\hline 64095 & Gpr35 & 1.11 & 0.68 & 0.69 \\
\hline 80910 & Gpr84 & 1.17 & 0.97 & 1.06 \\
\hline 84111 & Gpr87 & 1.09 & 0.87 & 0.96 \\
\hline 12766 & Cxcr3 & 1.08 & 1.01 & 1.30 \\
\hline 12801 & Cnr1 & 1.10 & 0.93 & 1.03 \\
\hline 13051 & Cx3cr1 & 1.16 & 0.94 & 1.03 \\
\hline 13488 & Drd1a & 1.13 & 1.15 & 1.23 \\
\hline 13490 & Drd3 & 1.16 & 1.08 & 1.20 \\
\hline 13491 & Drd4 & 1.16 & 0.85 & 1.09 \\
\hline 13609 & Edg1 & 1.17 & 0.99 & 1.30 \\
\hline 13617 & Ednra & 1.13 & 0.65 & 0.70 \\
\hline 14062 & F2r & 1.15 & 0.96 & 1.22 \\
\hline 14065 & F2rl3 & 1.14 & 1.04 & 1.28 \\
\hline 14293 & Fpr1 & 1.30 & 1.23 & 1.39 \\
\hline 14294 & Fprl1 & 1.12 & 1.00 & 1.19 \\
\hline 14527 & Gcgr & 1.18 & 0.78 & 0.82 \\
\hline 14602 & Ghrhr & 1.09 & 0.94 & 0.98 \\
\hline 14652 & Glp1r & 1.11 & 0.82 & 0.85 \\
\hline 14715 & Gnrhr & 1.15 & 0.95 & 1.03 \\
\hline 14747 & Cmklr1 & 1.16 & 0.87 & 1.04 \\
\hline 170757 & Eltd1 & 1.23 & 0.81 & 0.87 \\
\hline 226278 & Prlhr & 1.12 & 1.06 & 1.51 \\
\hline 227289 & Gpbar1 & 1.13 & 0.91 & 1.02 \\
\hline 229323 & Gpr171 & 1.14 & 0.78 & 0.93 \\
\hline 238252 & Gpr135 & 1.17 & 0.75 & 0.79 \\
\hline 239853 & Gpr128 & 1.17 & 0.85 & 0.85 \\
\hline
\end{tabular}




\begin{tabular}{|c|c|c|c|c|}
\hline 243277 & E230012M21 & 1.16 & 0.99 & 1.42 \\
\hline 269053 & Gpr152 & 1.16 & 0.90 & 0.98 \\
\hline 321019 & Ebi2 & 1.14 & 0.78 & 0.85 \\
\hline 52389 & D7Ertd680e & 1.16 & 0.96 & 1.29 \\
\hline 52614 & Emr4 & 1.11 & 1.06 & 1.34 \\
\hline 53978 & Edg4 & 1.08 & 1.08 & 1.36 \\
\hline 56696 & Gpr132 & 1.09 & 0.96 & 1.21 \\
\hline 78560 & Gpr124 & 1.28 & 1.10 & 1.10 \\
\hline 94226 & Edg8 & 1.18 & 1.19 & 1.53 \\
\hline 101533 & 1200016C12Rik & 1.06 & 0.94 & 1.01 \\
\hline 105501 & Abhd4 & 1.03 & 0.95 & 1.18 \\
\hline 11434 & Acr & 1.00 & 0.74 & 0.95 \\
\hline 11487 & Adam10 & 1.09 & 0.75 & 0.77 \\
\hline 11488 & Adam11 & 1.43 & 1.22 & 1.83 \\
\hline 11492 & Adam19 & 1.10 & 0.93 & 1.22 \\
\hline 11495 & Adam2 & 1.01 & 0.73 & 0.77 \\
\hline 13983 & Esr2 & 1.01 & 1.00 & 1.57 \\
\hline 15370 & Nr4a1 & 1.06 & 0.63 & 0.77 \\
\hline 171234 & V1rf3 & 1.00 & 0.92 & 1.57 \\
\hline 18227 & $\mathrm{Nr} 4 \mathrm{a} 2$ & 1.07 & 1.06 & 1.60 \\
\hline 18441 & P2ry1 & 1.10 & 0.45 & 0.52 \\
\hline 19214 & Ptgdr & 1.13 & 0.71 & 0.75 \\
\hline 19220 & Ptgfr & 1.06 & 0.48 & 0.59 \\
\hline 19222 & Ptgir & 1.01 & 0.80 & 1.15 \\
\hline 19228 & Pthr1 & 1.03 & 0.99 & 1.39 \\
\hline 19401 & Rara & 1.09 & 0.86 & 0.91 \\
\hline 20605 & Sstr1 & 1.03 & 0.76 & 0.87 \\
\hline 20607 & Sstr3 & 1.05 & 1.04 & 1.13 \\
\hline 20608 & Sstr4 & 1.06 & 0.68 & 0.96 \\
\hline 20609 & Sstr5 & 1.00 & 0.78 & 1.22 \\
\hline 21337 & Tacr2 & 1.01 & 0.86 & 1.03 \\
\hline 21338 & Tacr3 & 1.05 & 0.63 & 0.68 \\
\hline 213527 & Pthr2 & 1.06 & 1.05 & 1.80 \\
\hline 215854 & Taar5 & 1.04 & 0.82 & 1.11 \\
\hline 21833 & Thra & 1.22 & 0.87 & 1.01 \\
\hline 21834 & Thrb & 0.99 & 0.92 & 1.16 \\
\hline 21907 & $\mathrm{Nr} 2 \mathrm{e} 1$ & 1.06 & 0.44 & 0.50 \\
\hline 22045 & Trhr & 1.00 & 1.10 & 1.56 \\
\hline 22337 & Vdr & 1.11 & 0.59 & 0.60 \\
\hline 22354 & Vipr1 & 1.04 & 0.95 & 1.32 \\
\hline 22355 & Vipr2 & 1.03 & 0.76 & 0.95 \\
\hline 233571 & P2ry6 & 1.02 & 0.82 & 1.08 \\
\hline 23957 & NrOb2 & 1.06 & 1.01 & 1.41 \\
\hline 23958 & Nr2e3 & 1.10 & 0.49 & 0.58 \\
\hline 243083 & Tmprss11f & 1.11 & 0.84 & 1.06 \\
\hline 243084 & $\mathrm{~A} 030012 \mathrm{E} 10$ & 1.03 & 0.89 & 1.27 \\
\hline 319757 & Smo & 1.12 & 0.91 & 1.10 \\
\hline 329093 & Cpa6 & 1.09 & 0.90 & 1.15 \\
\hline 56544 & V2R2 & 1.05 & 0.77 & 1.13 \\
\hline 57385 & P2ry4 & 1.02 & 0.78 & 1.12 \\
\hline 66286 & 1810029G24Rik & 1.03 & 0.90 & 1.04 \\
\hline 67168 & P2y5 & 1.06 & 0.80 & 0.89 \\
\hline 67469 & Abhd5 & 1.06 & 0.88 & 1.12 \\
\hline 70008 & Ace2 & 1.11 & 0.77 & 0.93 \\
\hline
\end{tabular}




\begin{tabular}{|c|c|c|c|c|}
\hline 70839 & P2ry12 & 1.01 & 0.86 & 1.02 \\
\hline 72461 & 2510048K03Rik & 1.09 & 0.76 & 0.95 \\
\hline 73218 & 3110056003Rik & 1.04 & 1.07 & 1.31 \\
\hline 76453 & 2310046G15Rik & 1.12 & 0.71 & 0.82 \\
\hline 77593 & 4930550B20Rik & 1.04 & 0.81 & 0.86 \\
\hline 84112 & Sucnr1 & 1.02 & 0.88 & 1.23 \\
\hline 235606 & Apeh & 1.21 & 0.59 & 0.66 \\
\hline 23792 & Adam23 & 1.35 & 0.89 & 0.98 \\
\hline 70835 & Prss22 & 1.27 & 0.85 & 0.86 \\
\hline 12288 & Cacna1c & 1.16 & 0.78 & 0.82 \\
\hline 12298 & Cacnb4 & 1.11 & 0.86 & 0.90 \\
\hline 319446 & Dpep2 & 1.21 & 0.96 & 1.18 \\
\hline 11486 & Ada & 1.08 & 0.73 & 0.75 \\
\hline 21752 & Tert & 1.13 & 0.60 & 0.61 \\
\hline 12299 & Cacng1 & 1.13 & 0.90 & 1.08 \\
\hline 12337 & Capn5 & 1.09 & 0.87 & 0.90 \\
\hline 12864 & Cox6c & 1.07 & 0.96 & 1.00 \\
\hline 13860 & Eps8 & 1.24 & 0.95 & 0.99 \\
\hline 18606 & Enpp2 & 1.13 & 1.10 & 1.23 \\
\hline 353025 & Caps2 & 1.05 & 0.90 & 1.07 \\
\hline 50766 & Crim1 & 1.21 & 0.74 & 0.82 \\
\hline 74754 & Dhcr24 & 1.15 & 0.77 & 0.92 \\
\hline 13074 & Cyp17a1 & 1.03 & 0.95 & 1.03 \\
\hline 13075 & Cyp19a1 & 0.99 & 0.93 & 1.06 \\
\hline 217707 & Coq6 & 1.03 & 0.87 & 1.10 \\
\hline 22034 & Traf6 & 0.99 & 0.88 & 1.18 \\
\hline 22682 & Za20d2 & 1.04 & 0.62 & 0.75 \\
\hline 52700 & Txnl5 & 1.04 & 0.80 & 0.97 \\
\hline 53600 & Timm23 & 0.98 & 0.91 & 0.94 \\
\hline 69035 & Zdhhc3 & 0.99 & 0.48 & 0.67 \\
\hline 78903 & Wrnip1 & 1.05 & 0.80 & 0.84 \\
\hline 209027 & Pycr1 & 1.12 & 0.75 & 0.79 \\
\hline 102141 & Snx25 & 1.02 & 0.62 & 0.75 \\
\hline 103724 & Tbc1d10a & 1.03 & 0.79 & 0.81 \\
\hline 11848 & Rhoa & 1.04 & 0.84 & 1.01 \\
\hline 11853 & Rhoc & 1.02 & 0.81 & 1.08 \\
\hline 11858 & Rnd2 & 1.01 & 0.93 & 1.22 \\
\hline 14787 & Rhpn1 & 1.08 & 0.51 & 0.55 \\
\hline 19395 & Rasgrp2 & 1.01 & 0.75 & 0.90 \\
\hline 194590 & Reps2 & 1.08 & 0.77 & 0.85 \\
\hline 19731 & Rgl1 & 1.04 & 0.84 & 1.08 \\
\hline 19732 & Rgl2 & 1.16 & 0.80 & 0.86 \\
\hline 19734 & Rgs16 & 1.12 & 0.56 & 0.61 \\
\hline 19735 & Rgs2 & 1.17 & 0.87 & 0.94 \\
\hline 19737 & Rgs5 & 1.12 & 0.78 & 0.84 \\
\hline 19769 & Rit1 & 1.16 & 0.66 & 0.68 \\
\hline 20224 & Sar1a & 1.07 & 0.82 & 0.84 \\
\hline 20334 & Sec23a & 1.08 & 0.86 & 1.03 \\
\hline 20401 & Sh3bp1 & 1.13 & 0.89 & 0.94 \\
\hline 213391 & Rassf4 & 1.10 & 0.61 & 0.62 \\
\hline 215653 & Rassf2 & 1.02 & 0.75 & 0.76 \\
\hline 217463 & Snx13 & 1.07 & 0.62 & 0.69 \\
\hline 217692 & Sipa1l1 & 1.08 & 0.58 & 0.62 \\
\hline 21844 & Tiam1 & 1.09 & 0.91 & 1.15 \\
\hline
\end{tabular}




\begin{tabular}{|c|c|c|c|c|}
\hline 225870 & Rin1 & 1.07 & 0.62 & 0.76 \\
\hline 232441 & Rerg & 1.04 & 0.74 & 0.76 \\
\hline 233071 & Snx26 & 1.08 & 0.93 & 1.06 \\
\hline 233537 & 4921513004 & 1.05 & 0.77 & 0.85 \\
\hline 24012 & Rgs7 & 1.04 & 0.73 & 0.91 \\
\hline 246709 & Rgs13 & 1.03 & 0.75 & 0.89 \\
\hline 50778 & Rgs1 & 1.10 & 0.81 & 1.13 \\
\hline 51791 & Rgs14 & 1.10 & 0.60 & 0.72 \\
\hline 56212 & Rhog & 1.15 & 0.35 & 0.35 \\
\hline 56437 & Rrad & 1.02 & 0.65 & 0.80 \\
\hline 69159 & Rhebl1 & 1.08 & 0.54 & 0.60 \\
\hline 71330 & Rcbtb1 & 1.16 & 0.80 & 0.96 \\
\hline 72536 & Tagap & 1.14 & 0.76 & 0.85 \\
\hline 74156 & Cach & 1.05 & 0.84 & 0.98 \\
\hline 74194 & Rhoe & 1.08 & 0.84 & 1.00 \\
\hline 75985 & Rab30 & 1.14 & 0.38 & 0.39 \\
\hline 109904 & Mcf2 & 0.99 & 0.83 & 0.83 \\
\hline 114713 & Rasa2 & 1.08 & 0.69 & 0.75 \\
\hline 15463 & $\mathrm{Hrb}$ & 1.03 & 0.73 & 0.77 \\
\hline 16653 & Kras2 & 1.03 & 0.62 & 0.65 \\
\hline 18797 & Plcb3 & 1.04 & 0.74 & 0.81 \\
\hline 19330 & Rab18 & 1.02 & 0.90 & 0.97 \\
\hline 19337 & Rab33a & 1.03 & 0.82 & 0.84 \\
\hline 19338 & Rab33b & 1.16 & 0.96 & 1.11 \\
\hline 19414 & Rasa3 & 1.03 & 0.78 & 0.88 \\
\hline 19415 & Rasal1 & 1.11 & 0.75 & 0.84 \\
\hline 19416 & Rasd1 & 1.05 & 0.72 & 0.80 \\
\hline 212285 & LOC212285 & 1.04 & 0.87 & 1.05 \\
\hline 223864 & Rapgef3 & 1.04 & 0.93 & 0.96 \\
\hline 231801 & Agfg2 & 1.06 & 0.88 & 1.08 \\
\hline 270160 & Rab39 & 1.02 & 0.62 & 0.66 \\
\hline 271457 & Rab5a & 1.00 & 0.87 & 0.92 \\
\hline 67844 & Rab32 & 1.02 & 0.52 & 0.57 \\
\hline 74055 & Plce1 & 0.99 & 0.72 & 0.77 \\
\hline 103135 & Usp52 & 1.10 & 0.81 & 0.87 \\
\hline 103694 & Tmed4 & 1.06 & 0.92 & 0.97 \\
\hline 114886 & Cygb & 1.07 & 1.01 & 1.12 \\
\hline 11657 & Alb1 & 1.05 & 0.64 & 0.69 \\
\hline 13063 & Cycs & 1.06 & 0.85 & 0.96 \\
\hline 16803 & Lbp & 1.13 & 0.67 & 0.83 \\
\hline 16819 & Lcn2 & 1.08 & 0.83 & 0.84 \\
\hline 17189 & $\mathrm{Mb}$ & 1.03 & 0.67 & 0.73 \\
\hline 225651 & Mppe1 & 1.05 & 0.82 & 0.96 \\
\hline 330260 & Pon2 & 1.10 & 0.99 & 1.06 \\
\hline 50767 & Nte & 1.45 & 1.36 & 1.61 \\
\hline 71701 & Pnpt1 & 1.14 & 0.89 & 0.91 \\
\hline 74244 & Apg7I & 1.18 & 0.50 & 0.52 \\
\hline 78600 & Pde6h & 1.13 & 0.74 & 0.75 \\
\hline 12978 & Csf1r & 1.26 & 0.84 & 0.89 \\
\hline 16452 & Jak2 & 1.28 & 0.41 & 0.50 \\
\hline 16590 & Kit & 1.29 & 0.59 & 0.69 \\
\hline 57745 & Zfp112 & 1.18 & 0.87 & 1.10 \\
\hline 13713 & Elk3 & 1.01 & 0.75 & 0.75 \\
\hline 20254 & Scg2 & 1.06 & 0.91 & 0.92 \\
\hline
\end{tabular}




\begin{tabular}{|c|c|c|c|c|}
\hline 20351 & Sema4a & 1.05 & 0.73 & 0.93 \\
\hline 20358 & Sema6a & 1.03 & 0.71 & 1.02 \\
\hline 20394 & Scg5 & 1.05 & 0.45 & 0.46 \\
\hline 22402 & Wisp1 & 1.01 & 0.77 & 0.86 \\
\hline 224860 & Plcl2 & 1.03 & 0.86 & 0.87 \\
\hline 24117 & Wif1 & 1.06 & 0.89 & 1.01 \\
\hline 26456 & Sema4g & 1.03 & 0.47 & 0.49 \\
\hline 26556 & Homer1 & 1.03 & 0.80 & 0.81 \\
\hline 67196 & 2700084L22Rik & 1.08 & 0.77 & 0.80 \\
\hline 67405 & $\mathrm{Nts}$ & 1.05 & 0.85 & 0.95 \\
\hline 73318 & 1700013N18Rik & 1.06 & 0.92 & 1.00 \\
\hline 14609 & Gja1 & 1.09 & 0.90 & 0.99 \\
\hline 18417 & Cldn11 & 1.06 & 0.84 & 0.95 \\
\hline 20821 & Trim21 & 1.05 & 0.79 & 0.84 \\
\hline 22194 & Ube2e1 & 1.06 & 1.03 & 1.11 \\
\hline 22209 & Ube2a & 1.10 & 0.84 & 0.86 \\
\hline 26458 & Slc27a2 & 1.08 & 0.72 & 0.76 \\
\hline 54419 & Cldn6 & 1.10 & 0.91 & 1.06 \\
\hline 56228 & Ube2j1 & 1.11 & 0.95 & 1.06 \\
\hline 58187 & Cldn10 & 1.12 & 0.91 & 0.97 \\
\hline 66105 & Ube2d3 & 1.07 & 0.83 & 0.90 \\
\hline 66894 & Wwp2 & 1.05 & 0.66 & 0.77 \\
\hline 74153 & Ube1I & 1.06 & 0.83 & 0.92 \\
\hline 79263 & Trim39 & 1.05 & 0.83 & 0.87 \\
\hline 100705 & Acacb & 1.05 & 0.87 & 0.87 \\
\hline 107885 & Mthfs & 1.04 & 0.48 & 0.55 \\
\hline 17237 & Mgrn1 & 1.06 & 0.65 & 0.74 \\
\hline 18563 & Pcx & 1.11 & 0.85 & 0.87 \\
\hline 212085 & 4921513B05Rik & 1.06 & 0.81 & 0.94 \\
\hline 224826 & Ubr2 & 1.04 & 0.60 & 0.84 \\
\hline 229487 & Pet112I & 1.10 & 0.90 & 0.96 \\
\hline 59004 & Pias4 & 1.12 & 0.99 & 1.14 \\
\hline 97541 & Qars & 1.06 & 0.84 & 1.12 \\
\hline 99152 & Anapc2 & 1.04 & 0.87 & 1.01 \\
\hline $1 \mathrm{E}+08$ & Dnajc3 & 1.05 & 0.52 & 0.63 \\
\hline 13418 & Dnajc1 & 1.08 & 0.79 & 0.93 \\
\hline 14616 & Gja8 & 1.09 & 0.87 & 0.93 \\
\hline 14617 & Gjd2 & 1.06 & 0.85 & 0.91 \\
\hline 15507 & Hspb1 & 1.07 & 0.96 & 1.16 \\
\hline 15528 & Hspe1 & 1.11 & 0.89 & 1.14 \\
\hline 17475 & Mpdz & 1.03 & 1.00 & 1.00 \\
\hline 17714 & Grpel2 & 1.05 & 0.78 & 0.87 \\
\hline 213539 & Bag2 & 1.04 & 0.92 & 0.93 \\
\hline 231997 & Fkbp14 & 1.07 & 0.74 & 0.97 \\
\hline 56354 & Dnajc7 & 1.07 & 0.59 & 0.84 \\
\hline 56445 & Dnaja2 & 1.03 & 0.83 & 1.02 \\
\hline 57431 & Dnajc4 & 1.06 & 0.68 & 0.87 \\
\hline 64010 & Sav1 & 1.03 & 0.93 & 1.14 \\
\hline 66411 & Ckap1 & 1.07 & 0.93 & 1.03 \\
\hline 67035 & Dnajb4 & 1.04 & 0.99 & 1.02 \\
\hline 68598 & Dnajc8 & 1.07 & 0.75 & 1.14 \\
\hline 76889 & Adck4 & 1.06 & 0.95 & 0.99 \\
\hline 80888 & Hspb8 & 1.08 & 0.97 & 1.00 \\
\hline 83945 & Dnaja3 & 1.07 & 0.80 & 0.98 \\
\hline
\end{tabular}




\begin{tabular}{|c|c|c|c|c|}
\hline 93742 & Pard3 & 1.03 & 0.91 & 0.94 \\
\hline 23970 & Pacsin2 & 1.06 & 0.61 & 0.89 \\
\hline 386649 & Nsfl1c & 1.05 & 0.75 & 0.76 \\
\hline 56440 & Snx1 & 1.04 & 0.89 & 0.91 \\
\hline 71889 & Epn3 & 1.05 & 0.82 & 0.88 \\
\hline 11629 & Aif1 & 1.15 & 0.72 & 0.78 \\
\hline 12813 & Col10a1 & 1.07 & 0.82 & 0.88 \\
\hline 18125 & Nos1 & 1.11 & 0.64 & 0.71 \\
\hline 18126 & Nos2 & 1.10 & 0.60 & 0.71 \\
\hline 18570 & Pdcd6 & 1.11 & 0.47 & 0.50 \\
\hline 20193 & S100a1 & 1.08 & 0.72 & 0.79 \\
\hline 20198 & S100a4 & 1.08 & 0.83 & 0.86 \\
\hline 21926 & Tnf & 1.09 & 0.52 & 0.54 \\
\hline 270084 & Lpcat2 & 1.16 & 0.76 & 0.92 \\
\hline 27062 & Cadps & 1.09 & 0.88 & 0.89 \\
\hline 52589 & Ncald & 1.09 & 0.72 & 0.83 \\
\hline 67500 & Ccar1 & 1.12 & 0.71 & 0.72 \\
\hline 70527 & Stambp & 1.08 & 0.86 & 0.90 \\
\hline 73316 & Calr3 & 1.11 & 0.92 & 0.96 \\
\hline 80796 & Calm4 & 1.12 & 0.81 & 1.02 \\
\hline 12263 & C2 & 1.10 & 0.83 & 0.91 \\
\hline 14134 & Fcnb & 1.10 & 0.64 & 0.74 \\
\hline 14256 & Flt3l & 1.07 & 0.51 & 0.68 \\
\hline 14962 & Cfb & 1.13 & 0.65 & 0.87 \\
\hline 16168 & II15 & 1.09 & 0.56 & 0.60 \\
\hline 20292 & Ccl11 & 1.11 & 0.92 & 0.93 \\
\hline 50908 & C1s & 1.08 & 0.86 & 0.91 \\
\hline 50928 & KIrg1 & 1.09 & 0.85 & 0.86 \\
\hline 54525 & Syt7 & 1.10 & 0.79 & 0.83 \\
\hline 14118 & Fbn1 & 1.19 & 0.91 & 1.00 \\
\hline 16782 & Lamc2 & 1.19 & 1.06 & 1.17 \\
\hline 17388 & Mmp15 & 1.23 & 0.99 & 1.12 \\
\hline 17392 & Mmp3 & 1.18 & 0.88 & 1.22 \\
\hline 17395 & Mmp9 & 1.21 & 0.99 & 1.11 \\
\hline 18074 & Nid2 & 1.20 & 1.08 & 1.31 \\
\hline 214766 & Mmp21 & 1.25 & 0.68 & 0.92 \\
\hline 23948 & Mmp17 & 1.27 & 0.78 & 1.10 \\
\hline 240047 & Mmp25 & 1.21 & 0.69 & 0.92 \\
\hline 26561 & Mmp23 & 1.19 & 0.81 & 1.06 \\
\hline 13433 & Dnmt1 & 1.04 & 0.92 & 1.09 \\
\hline 13629 & Eef2 & 1.05 & 0.84 & 0.94 \\
\hline 13685 & Eif4ebp1 & 1.02 & 0.78 & 0.93 \\
\hline 13690 & Eif4g2 & 1.07 & 0.72 & 0.81 \\
\hline 13796 & Emx1 & 1.05 & 0.77 & 1.08 \\
\hline 13797 & Emx2 & 1.02 & 0.74 & 0.75 \\
\hline 14265 & Fmr1 & 1.01 & 0.80 & 1.09 \\
\hline 14836 & Gsc & 1.07 & 0.82 & 1.03 \\
\hline 14842 & Gsh1 & 1.07 & 0.73 & 0.75 \\
\hline 14843 & Gsh2 & 1.05 & 0.93 & 1.04 \\
\hline 14852 & Gspt1 & 1.07 & 0.86 & 0.97 \\
\hline 15115 & Hars & 1.06 & 0.87 & 0.92 \\
\hline 15248 & Hic1 & 1.05 & 0.85 & 0.98 \\
\hline 212528 & Trmt1 & 1.03 & 0.97 & 0.98 \\
\hline 226414 & Dars & 1.03 & 0.73 & 0.85 \\
\hline
\end{tabular}




\begin{tabular}{|c|c|c|c|c|}
\hline 23879 & Fxr2 & 1.02 & 0.67 & 0.68 \\
\hline 54632 & Ftsj1 & 1.01 & 0.78 & 1.00 \\
\hline 70791 & Hars2 & 1.07 & 0.65 & 0.76 \\
\hline 15312 & Hmgn1 & 1.00 & 0.80 & 0.84 \\
\hline 16589 & Uhmk1 & 1.02 & 0.88 & 1.07 \\
\hline 171170 & Mbnl3 & 1.05 & 0.68 & 0.78 \\
\hline 70223 & Nars & 1.02 & 0.62 & 0.70 \\
\hline 72199 & Mms19l & 1.03 & 0.72 & 0.82 \\
\hline 76936 & Hnrpm & 1.03 & 0.82 & 0.91 \\
\hline 94353 & Hmgn3 & 1.01 & 0.81 & 0.89 \\
\hline 108017 & Fxyd4 & 1.22 & 0.83 & 0.96 \\
\hline 171209 & Accn3 & 1.19 & 0.82 & 0.86 \\
\hline 17178 & Fxyd3 & 1.22 & 0.77 & 0.82 \\
\hline 217356 & Tmc8 & 1.19 & 0.95 & 1.06 \\
\hline 229927 & Clca4 & 1.20 & 1.15 & 1.46 \\
\hline 435845 & Tmprss11c & 1.17 & 0.73 & 0.74 \\
\hline 56808 & Cacna2d2 & 1.20 & 0.90 & 1.04 \\
\hline 74270 & Usp20 & 1.18 & 0.71 & 0.95 \\
\hline 103710 & Slc35e4 & 1.03 & 0.95 & 1.16 \\
\hline 106039 & Gga1 & 1.01 & 0.95 & 1.30 \\
\hline 110891 & Slc8a2 & 1.00 & 0.83 & 0.93 \\
\hline 117591 & Slc2a9 & 1.10 & 0.81 & 1.30 \\
\hline 140918 & Slc7a12 & 1.05 & 0.97 & 1.13 \\
\hline 142681 & Slc34a3 & 1.02 & 0.72 & 1.27 \\
\hline 14664 & Slc6a9 & 1.07 & 0.95 & 1.14 \\
\hline 16792 & Laptm5 & 1.03 & 1.02 & 1.34 \\
\hline 18399 & Slc22a6 & 1.00 & 0.96 & 1.28 \\
\hline 20509 & Slc19a1 & 1.03 & 1.05 & 1.25 \\
\hline 20536 & Slc4a3 & 1.07 & 0.95 & 1.05 \\
\hline 207151 & Slc22a9 & 1.00 & 1.05 & 1.32 \\
\hline 210148 & Slc30a6 & 1.01 & 0.75 & 1.17 \\
\hline 216867 & Slc16a11 & 1.02 & 0.90 & 1.16 \\
\hline 224661 & Slc26a8 & 1.01 & 1.00 & 1.13 \\
\hline 22599 & Slc6a20b & 1.03 & 0.94 & 1.08 \\
\hline 227059 & Slc39a10 & 1.03 & 1.02 & 1.33 \\
\hline 229706 & Slc6a17 & 1.12 & 0.94 & 1.04 \\
\hline 242773 & Slc45a1 & 1.01 & 0.91 & 1.15 \\
\hline 260302 & Gga3 & 1.05 & 0.98 & 1.25 \\
\hline 268512 & Slc26a11 & 1.04 & 0.97 & 1.06 \\
\hline 54160 & Copg2 & 1.00 & 0.99 & 1.36 \\
\hline 56358 & Copz2 & 1.02 & 1.00 & 1.27 \\
\hline 59042 & Cope & 1.02 & 0.98 & 1.27 \\
\hline 64454 & Slc5a4b & 1.01 & 0.99 & 1.38 \\
\hline 67843 & Slc35a4 & 1.00 & 0.67 & 1.19 \\
\hline 69089 & Oxa11 & 1.01 & 0.89 & 1.26 \\
\hline 71805 & Nup93 & 1.00 & 1.03 & 1.53 \\
\hline 72027 & Slc39a4 & 1.01 & 0.94 & 1.16 \\
\hline 72961 & Slc17a7 & 1.01 & 1.03 & 1.46 \\
\hline 74102 & Slc35a5 & 1.03 & 0.67 & 1.24 \\
\hline 74338 & Slc6a19 & 1.06 & 1.07 & 1.23 \\
\hline 77996 & D730039F16Rik & 1.07 & 0.88 & 1.20 \\
\hline 98396 & Slc41a1 & 1.00 & 1.03 & 1.20 \\
\hline 108956 & 2210421G13Rik & 0.99 & 1.01 & 1.13 \\
\hline 109280 & 9330176C04Rik & 1.05 & 0.93 & 1.25 \\
\hline
\end{tabular}




\begin{tabular}{|c|c|c|c|c|}
\hline 11777 & Ap3s1 & 1.05 & 0.92 & 1.36 \\
\hline 16531 & Kcnma1 & 1.05 & 0.96 & 1.12 \\
\hline 16532 & Kcnma3 & 1.01 & 0.95 & 1.11 \\
\hline 16536 & Kcnq2 & 1.02 & 0.96 & 1.10 \\
\hline 18669 & Abcb1b & 1.00 & 0.63 & 1.15 \\
\hline 18670 & Abcb4 & 1.01 & 0.68 & 1.24 \\
\hline 192140 & Tmc2 & 1.01 & 0.93 & 1.29 \\
\hline 193034 & Trpv1 & 1.01 & 0.90 & 1.01 \\
\hline 20271 & Scn5a & 1.00 & 0.97 & 1.09 \\
\hline 20928 & Abcc9 & 0.99 & 0.56 & 1.11 \\
\hline 210463 & BC026439 & 1.07 & 1.09 & 1.19 \\
\hline 213603 & BC010552 & 1.07 & 0.93 & 1.18 \\
\hline 213827 & Arcn1 & 1.00 & 0.95 & 1.17 \\
\hline 223604 & Kcnk9 & 1.04 & 0.92 & 1.11 \\
\hline 224742 & Abcf1 & 1.00 & 0.57 & 0.91 \\
\hline 228993 & BC019537 & 1.00 & 0.76 & 0.94 \\
\hline 231430 & $\mathrm{BC} 038311$ & 1.05 & 0.97 & 1.08 \\
\hline 232910 & Ap2s1 & 1.00 & 1.07 & 1.55 \\
\hline 236149 & Slc22a26 & 1.05 & 0.91 & 1.23 \\
\hline 241612 & Slc5a12 & 0.99 & 0.70 & 1.05 \\
\hline 241919 & BC061928 & 1.02 & 0.81 & 0.81 \\
\hline 243813 & Leng9 & 1.05 & 0.95 & 1.01 \\
\hline 252903 & Ap1s3 & 0.99 & 0.93 & 1.23 \\
\hline 27061 & Bcap31 & 1.02 & 1.02 & 1.22 \\
\hline 319800 & C730048C13Rik & 1.06 & 0.92 & 1.18 \\
\hline 320590 & 9430071P14Rik & 1.01 & 0.98 & 1.36 \\
\hline 338363 & 6030446N20Rik & 0.99 & 0.89 & 1.19 \\
\hline 338365 & A230035L05Rik & 1.00 & 0.91 & 1.35 \\
\hline 399548 & Scn $4 b$ & 1.01 & 0.93 & 1.17 \\
\hline 56325 & Abcb9 & 0.99 & 0.61 & 1.25 \\
\hline 56334 & Tmed2 & 0.99 & 0.95 & 1.09 \\
\hline 57776 & Ttyh1 & 1.03 & 0.92 & 1.07 \\
\hline 67135 & 2310021H06Rik & 1.03 & 1.15 & 1.18 \\
\hline 68044 & 2510006C20Rik & 0.99 & 0.97 & 1.57 \\
\hline 68279 & Mcoln2 & 1.00 & 0.93 & 1.00 \\
\hline 68682 & 1110028E10Rik & 1.02 & 0.93 & 1.34 \\
\hline 69065 & 1810008K03Rik & 1.00 & 1.03 & 1.29 \\
\hline 72175 & 2810423E13Rik & 1.00 & 0.95 & 1.30 \\
\hline 74424 & Tmc5 & 1.00 & 0.97 & 1.30 \\
\hline 75761 & Apol7a & 1.00 & 0.93 & 0.99 \\
\hline 77577 & 9830002I17Rik & 0.99 & 0.85 & 1.15 \\
\hline 101744 & C330005M16Rik & 1.05 & 0.89 & 0.93 \\
\hline 102954 & Nudt10 & 1.00 & 1.06 & 1.16 \\
\hline 105352 & Dusp22 & 1.04 & 0.63 & 1.06 \\
\hline 108096 & Slco1a5 & 1.04 & 0.98 & 1.04 \\
\hline 110074 & Dutp & 1.02 & 0.65 & 1.03 \\
\hline 110332 & 4921523A10Rik & 1.02 & 1.02 & 1.20 \\
\hline 110895 & Slc9a4 & 1.07 & 0.93 & 1.14 \\
\hline 13853 & Epm2a & 1.02 & 0.65 & 1.13 \\
\hline 14198 & Fhit & 1.04 & 0.91 & 1.07 \\
\hline 16331 & Inpp5d & 1.03 & 1.01 & 1.11 \\
\hline 170835 & Pib5pa & 1.06 & 0.96 & 1.09 \\
\hline 18640 & Pfkfb2 & 1.02 & 1.09 & 1.24 \\
\hline 214901 & Chtf18 & 1.01 & 0.82 & 0.88 \\
\hline
\end{tabular}




\begin{tabular}{|c|c|c|c|c|}
\hline 216152 & BC005764 & 1.01 & 1.11 & 1.49 \\
\hline 232941 & C79127 & 1.00 & 1.05 & 1.25 \\
\hline 235534 & Acpl2 & 1.04 & 0.95 & 1.15 \\
\hline 242291 & 1110001C20Rik & 1.02 & 1.10 & 1.28 \\
\hline 28248 & Slco1a1 & 1.01 & 0.94 & 1.12 \\
\hline 28250 & Slco1a4 & 1.03 & 0.86 & 1.00 \\
\hline 28254 & Slco1a6 & 1.04 & 1.06 & 1.12 \\
\hline 384619 & Ccdc155 & 1.07 & 1.05 & 1.42 \\
\hline 52036 & Saps3 & 1.01 & 0.73 & 1.32 \\
\hline 54561 & Nap1I3 & 1.03 & 0.96 & 1.06 \\
\hline 57028 & Pdxp & 1.01 & 1.00 & 1.11 \\
\hline 58242 & Nudt11 & 1.03 & 0.98 & 1.16 \\
\hline 58243 & Nap115 & 1.01 & 1.06 & 1.22 \\
\hline 60600 & Tsga8 & 1.02 & 0.87 & 0.93 \\
\hline 66387 & Nudt8 & 1.02 & 1.02 & 1.13 \\
\hline 67395 & 4930403L05Rik & 1.01 & 1.07 & 1.24 \\
\hline 67528 & Nudt7 & 1.00 & 1.04 & 1.45 \\
\hline 67952 & Tomm20 & 1.00 & 1.07 & 1.26 \\
\hline 68695 & 1110033009Rik & 1.02 & 0.98 & 1.29 \\
\hline 69274 & Ctdspl & 1.01 & 0.78 & 1.24 \\
\hline 71474 & Ppp6r2 & 1.00 & 0.91 & 1.21 \\
\hline 74140 & Tm9sf1 & 1.04 & 0.93 & 1.20 \\
\hline 74189 & Phactr3 & 1.05 & 1.10 & 1.37 \\
\hline 74411 & 4932443D16Rik & 1.00 & 1.00 & 1.12 \\
\hline 75769 & 4833424015Rik & 1.10 & 1.05 & 1.42 \\
\hline 77573 & Vps33a & 1.00 & 0.87 & 0.96 \\
\hline 13057 & Cyba & 1.00 & 0.80 & 0.82 \\
\hline 217864 & D12Wsu95e & 1.04 & 0.81 & 0.84 \\
\hline 276829 & Smtnl2 & 1.03 & 0.77 & 0.80 \\
\hline 319593 & D130011D22Rik & 1.01 & 0.91 & 0.92 \\
\hline 320373 & D130016K21Rik & 1.01 & 0.76 & 0.76 \\
\hline 330577 & D030069K18 & 1.03 & 0.95 & 0.97 \\
\hline 442805 & D130007C19Rik & 1.01 & 0.87 & 0.90 \\
\hline 52014 & Nus1 & 1.00 & 0.92 & 0.97 \\
\hline 52668 & Ifi27l1 & 1.00 & 0.79 & 0.91 \\
\hline 52670 & Cpsf4l & 1.02 & 0.95 & 1.11 \\
\hline 52717 & D10Ertd641e & 1.03 & 0.84 & 0.98 \\
\hline 214932 & Cecr5 & 1.04 & 0.89 & 1.04 \\
\hline 225745 & Ccdc5 & 1.08 & 0.88 & 1.10 \\
\hline 321022 & Cdv3 & 1.03 & 0.87 & 0.91 \\
\hline 57912 & Cdc42se1 & 1.04 & 0.91 & 0.97 \\
\hline 66440 & Cdc26 & 1.07 & 0.79 & 0.83 \\
\hline 67849 & Cdca5 & 1.06 & 1.10 & 1.20 \\
\hline 71991 & Ckn1 & 1.07 & 0.93 & 1.00 \\
\hline 72278 & Ccpg1 & 1.09 & 1.07 & 1.08 \\
\hline 94040 & Clmn & 1.14 & 1.00 & 1.06 \\
\hline 103466 & C630002B14Rik & 1.05 & 0.97 & 1.09 \\
\hline 12331 & Cap1 & 1.00 & 0.86 & 0.89 \\
\hline 231855 & Ap5z1 & 1.09 & 0.88 & 0.97 \\
\hline 235386 & C630028N24Rik & 1.03 & 0.80 & 0.89 \\
\hline 442802 & C330011M18Rik & 1.01 & 0.92 & 0.93 \\
\hline 54378 & Cacng6 & 1.02 & 0.79 & 0.81 \\
\hline 68112 & C330016H24Rik & 1.04 & 0.82 & 0.82 \\
\hline 12959 & Cryba4 & 1.07 & 0.97 & 1.12 \\
\hline
\end{tabular}




\begin{tabular}{|c|c|c|c|c|}
\hline 12964 & Cryga & 1.08 & 0.85 & 0.99 \\
\hline 12967 & Crygd & 1.03 & 1.03 & 1.05 \\
\hline 12970 & Crygs & 1.04 & 1.05 & 1.07 \\
\hline 234577 & Cpne2 & 1.06 & 0.97 & 1.17 \\
\hline 245684 & Cnksr2 & 1.05 & 0.83 & 0.92 \\
\hline 266692 & Cpne1 & 1.04 & 1.05 & 1.13 \\
\hline 338337 & Cog3 & 1.08 & 1.00 & 1.16 \\
\hline 57358 & Cmar & 1.03 & 0.94 & 1.03 \\
\hline 66398 & Commd5 & 1.10 & 1.04 & 1.18 \\
\hline 76524 & Cln6 & 1.09 & 0.90 & 0.97 \\
\hline 94109 & Csmd1 & 1.04 & 1.05 & 1.05 \\
\hline 13626 & Eed & 1.07 & 0.95 & 1.03 \\
\hline 210757 & E430004N04Rik & 1.04 & 0.98 & 1.01 \\
\hline 211305 & E330017E16 & 1.04 & 1.03 & 1.12 \\
\hline 228598 & Ebf4 & 1.04 & 0.94 & 1.03 \\
\hline 240754 & Lax1 & 1.07 & 1.05 & 1.16 \\
\hline 319996 & D130060C09Rik & 1.12 & 1.10 & 1.19 \\
\hline 320360 & E130307J04Rik & 1.06 & 1.00 & 1.08 \\
\hline 403205 & E030025L21Rik & 1.05 & 0.93 & 1.18 \\
\hline 54648 & Ccdc120 & 1.04 & 1.07 & 1.20 \\
\hline 231630 & D5Ertd40e & 1.06 & 0.93 & 1.01 \\
\hline 65111 & Dap3 & 1.06 & 0.88 & 0.97 \\
\hline 213491 & Szrd1 & 1.09 & 0.86 & 0.95 \\
\hline 219072 & Haus4 & 1.08 & 0.86 & 0.92 \\
\hline 232813 & D430041B17 & 1.06 & 0.85 & 0.99 \\
\hline 27762 & Vwa7 & 1.07 & 0.97 & 1.09 \\
\hline 319388 & D230002A01Rik & 1.08 & 0.97 & 1.03 \\
\hline 319655 & Podxl2 & 1.07 & 0.99 & 1.14 \\
\hline 52552 & D13Ertd275e & 1.14 & 1.01 & 1.06 \\
\hline 52846 & D1Bwg0212e & 1.05 & 0.85 & 0.89 \\
\hline 67586 & D4Bwg1540e & 1.06 & 0.94 & 1.10 \\
\hline 67948 & Fbxo28 & 1.09 & 1.04 & 1.17 \\
\hline 78581 & D530033C11Rik & 1.12 & 0.99 & 1.19 \\
\hline 107986 & Ddb2 & 1.05 & 1.02 & 1.02 \\
\hline 112403 & Dom3z & 1.07 & 1.05 & 1.15 \\
\hline 116905 & Dph2l1 & 1.15 & 0.95 & 1.03 \\
\hline 13216 & Defa1 & 1.07 & 1.09 & 1.27 \\
\hline 13221 & Defa-rs12 & 1.09 & 1.06 & 1.18 \\
\hline 13238 & Defa4 & 1.07 & 0.96 & 1.13 \\
\hline 13239 & Defcr5 & 1.07 & 1.02 & 1.23 \\
\hline 13380 & Dkk1 & 1.13 & 1.01 & 1.22 \\
\hline 13446 & Doc2a & 1.08 & 1.06 & 1.13 \\
\hline 13447 & Doc2b & 1.06 & 1.04 & 1.14 \\
\hline 13517 & Dspp & 1.10 & 1.01 & 1.20 \\
\hline 13518 & Dst & 1.08 & 1.08 & 1.15 \\
\hline 227525 & Dclre1c & 1.18 & 1.02 & 1.11 \\
\hline 23853 & Def6 & 1.09 & 0.93 & 1.10 \\
\hline 23854 & Def8 & 1.09 & 0.99 & 1.10 \\
\hline 246079 & Defb9 & 1.09 & 1.02 & 1.24 \\
\hline 360212 & Defb38 & 1.08 & 0.98 & 1.00 \\
\hline 50722 & Dkkl1 & 1.11 & 1.20 & 1.38 \\
\hline 54638 & DXIm $\times 40 \mathrm{e}$ & 1.25 & 1.04 & 1.44 \\
\hline 54722 & Dfna5h & 1.19 & 1.18 & 1.36 \\
\hline 56176 & Pigp & 1.07 & 0.95 & 0.99 \\
\hline
\end{tabular}




\begin{tabular}{|c|c|c|c|c|}
\hline 56455 & Dnclc1 & 1.08 & 1.19 & 1.26 \\
\hline 56811 & Dkk2 & 1.11 & 0.95 & 1.19 \\
\hline 58251 & Ddc8 & 1.10 & 1.00 & 1.08 \\
\hline 66148 & Dnajc15 & 1.06 & 0.99 & 1.15 \\
\hline 67379 & Dedd2 & 1.11 & 1.02 & 1.03 \\
\hline 67728 & Dph2l2 & 1.06 & 1.05 & 1.14 \\
\hline 68184 & Denr & 1.06 & 0.99 & 1.24 \\
\hline 68563 & Dpm3 & 1.07 & 1.20 & 1.41 \\
\hline 68897 & Disp1 & 1.10 & 1.06 & 1.07 \\
\hline 71804 & Dufd1 & 1.11 & 1.01 & 1.06 \\
\hline 71972 & Dnmbp & 1.11 & 1.04 & 1.30 \\
\hline 73284 & Ddit4I & 1.13 & 1.00 & 1.02 \\
\hline 77674 & Defb12 & 1.16 & 1.06 & 1.29 \\
\hline 94223 & Dgcr8 & 1.08 & 1.22 & 1.30 \\
\hline 97998 & Deptor & 1.06 & 1.08 & 1.10 \\
\hline 102920 & Fshprh1 & 1.12 & 0.85 & 1.08 \\
\hline 103583 & Fbxw11 & 1.14 & 0.79 & 0.89 \\
\hline 14154 & Fem1a & 1.05 & 0.67 & 0.73 \\
\hline 14210 & Fin15 & 1.10 & 0.90 & 0.99 \\
\hline 14221 & Fjx1 & 1.09 & 0.89 & 1.07 \\
\hline 14352 & Fv4 & 1.07 & 0.68 & 0.74 \\
\hline 17281 & Fyco1 & 1.10 & 0.91 & 0.94 \\
\hline 213980 & Fbxw10 & 1.10 & 0.69 & 0.71 \\
\hline 231470 & Fras1 & 1.05 & 0.97 & 1.14 \\
\hline 239839 & Ccdc14 & 1.21 & 0.68 & 0.79 \\
\hline 240263 & Fem1c & 1.06 & 0.88 & 0.94 \\
\hline 30050 & Fbxw2 & 1.10 & 0.84 & 0.95 \\
\hline 384061 & Fndc5 & 1.06 & 0.87 & 0.98 \\
\hline 50759 & Fbxo16 & 1.07 & 0.79 & 0.81 \\
\hline 50764 & Fbxo15 & 1.16 & 0.99 & 1.05 \\
\hline 64339 & Fndc4 & 1.08 & 0.75 & 0.83 \\
\hline 66153 & Fbxo36 & 1.07 & 0.91 & 1.08 \\
\hline 71313 & Fsip1 & 1.05 & 0.77 & 1.04 \\
\hline 78938 & Fbxo34 & 1.19 & 0.75 & 0.81 \\
\hline 219150 & F830020C16Rik & 1.13 & 0.82 & 0.91 \\
\hline 320463 & F630111L10Rik & 1.10 & 0.93 & 0.97 \\
\hline 50757 & Fbxo12 & 1.17 & 0.84 & 0.90 \\
\hline 54357 & Epb4.114b & 1.11 & 0.67 & 0.67 \\
\hline 14585 & Gfra1 & 1.09 & 1.07 & 1.11 \\
\hline 217648 & Gm527 & 1.05 & 0.87 & 0.90 \\
\hline 229588 & Gm128 & 1.06 & 0.95 & 0.96 \\
\hline 23885 & $\mathrm{Gcl}$ & 1.15 & 0.89 & 1.10 \\
\hline 241950 & Gm1805 & 1.09 & 0.83 & 0.87 \\
\hline 381334 & Gal3st2 & 1.26 & 0.80 & 1.04 \\
\hline 68592 & Gcipip & 1.20 & 0.67 & 0.80 \\
\hline 70772 & Ggnbp1 & 1.09 & 0.87 & 0.97 \\
\hline 93683 & Glce & 1.08 & 0.91 & 0.97 \\
\hline 16970 & Lrmp & 1.13 & 0.80 & 0.86 \\
\hline 211228 & Lrrc25 & 1.56 & 1.08 & 1.10 \\
\hline 269593 & Luzp1 & 1.13 & 0.97 & 0.99 \\
\hline 432582 & E130309D14Rik & 1.11 & 0.92 & 0.99 \\
\hline 432589 & LOC432589 & 1.11 & 0.93 & 0.96 \\
\hline 544988 & 544988 & 1.12 & 0.83 & 0.85 \\
\hline 16639 & KIra8 & 1.08 & 1.08 & 1.09 \\
\hline
\end{tabular}




\begin{tabular}{|c|c|c|c|c|}
\hline 67224 & Med29 & 1.06 & 0.81 & 0.94 \\
\hline 140559 & Igsf8 & 1.14 & 1.24 & 1.26 \\
\hline 15982 & Ifrd1 & 1.02 & 1.09 & 1.18 \\
\hline 15983 & Ifrd2 & 1.04 & 1.14 & 1.26 \\
\hline 192232 & Hps4 & 1.17 & 1.42 & 1.48 \\
\hline 382522 & Hist3h2bb-ps & 1.01 & 1.18 & 1.21 \\
\hline 66141 & Ifitm3 & 1.11 & 1.39 & 1.51 \\
\hline 66320 & Tmem208 & 0.98 & 1.14 & 1.17 \\
\hline 66667 & Hspbap1 & 1.06 & 1.00 & 1.09 \\
\hline 80876 & Ifitm2 & 1.01 & 1.34 & 1.38 \\
\hline 83408 & Gimap3 & 0.99 & 1.07 & 1.09 \\
\hline 99899 & Ifi44 & 1.04 & 0.84 & 0.88 \\
\hline 13863 & Lcn5 & 1.02 & 0.80 & 0.83 \\
\hline 16699 & Krtap13 & 1.04 & 0.74 & 0.90 \\
\hline 212871 & LOC212871 & 1.14 & 0.81 & 0.87 \\
\hline 268905 & Krtap13-1 & 1.11 & 0.82 & 0.86 \\
\hline 381806 & LOC381806 & 1.03 & 0.68 & 0.72 \\
\hline 432555 & LOC432555 & 1.03 & 0.64 & 0.71 \\
\hline 54135 & Lsr & 1.08 & 0.94 & 1.08 \\
\hline 66380 & Krtap3-3 & 1.05 & 0.87 & 0.99 \\
\hline 66708 & Krtap3-2 & 1.02 & 0.75 & 0.88 \\
\hline 76893 & Lass2 & 1.05 & 0.86 & 0.90 \\
\hline 18095 & Nkx3-1 & 1.09 & 0.69 & 0.72 \\
\hline 18307 & Olfr10 & 1.13 & 0.90 & 1.17 \\
\hline 213765 & BC125332 & 1.09 & 0.88 & 1.00 \\
\hline 259015 & Olfr1038 & 1.08 & 0.84 & 0.91 \\
\hline 433904 & Ociad2 & 1.11 & 0.66 & 0.72 \\
\hline 67122 & Nrarp & 1.08 & 0.58 & 0.75 \\
\hline 68039 & $\mathrm{Nmb}$ & 1.07 & 0.71 & 0.72 \\
\hline 72310 & Nkg7 & 1.07 & 0.60 & 0.73 \\
\hline 258925 & Olfr20 & 1.07 & 0.76 & 0.81 \\
\hline 28028 & Mrpl50 & 1.07 & 0.72 & 0.76 \\
\hline 434204 & MGC51670 & 1.05 & 0.90 & 0.92 \\
\hline 68499 & Mrpl53 & 1.04 & 0.75 & 0.89 \\
\hline 17149 & Magoh & 1.09 & 0.69 & 0.71 \\
\hline 17235 & Mcsp & 1.06 & 0.60 & 0.66 \\
\hline 66591 & Mad2l1bp & 1.07 & 0.94 & 0.96 \\
\hline 170829 & Tram2 & 0.98 & 0.84 & 1.01 \\
\hline 219024 & Tmem55b & 0.98 & 0.62 & 0.72 \\
\hline 21950 & Tnfsf9 & 1.04 & 0.65 & 0.73 \\
\hline 22031 & Traf3 & 1.01 & 0.72 & 0.77 \\
\hline 22040 & Trex1 & 0.96 & 0.82 & 0.87 \\
\hline 22057 & Tob1 & 0.98 & 0.79 & 0.81 \\
\hline 22157 & Tulp1 & 0.98 & 0.78 & 0.87 \\
\hline 224840 & Treml4 & 0.97 & 0.71 & 0.76 \\
\hline 227331 & Tnrc15 & 1.02 & 0.82 & 0.85 \\
\hline 227606 & Tbpl2 & 0.96 & 0.73 & 0.76 \\
\hline 231130 & Tnip2 & 1.00 & 0.79 & 0.83 \\
\hline 252838 & Tox & 0.98 & 0.69 & 0.70 \\
\hline 27279 & Tnfrsf12a & 0.95 & 0.76 & 0.79 \\
\hline 277414 & Trp53i11 & 0.99 & 0.73 & 0.83 \\
\hline 30934 & Tor1b & 0.96 & 0.86 & 0.96 \\
\hline 64930 & Tsc1 & 0.95 & 0.83 & 0.83 \\
\hline 66241 & Tmem9 & 0.96 & 0.65 & 0.67 \\
\hline
\end{tabular}




\begin{tabular}{|c|c|c|c|c|}
\hline 67946 & Spata6 & 1.01 & 0.62 & 0.66 \\
\hline 71326 & Treml1 & 1.00 & 0.70 & 0.83 \\
\hline 71609 & Tradd & 1.00 & 0.72 & 0.74 \\
\hline 72236 & Tsnaxip1 & 1.01 & 0.74 & 0.74 \\
\hline 72265 & Tram1 & 0.98 & 0.77 & 0.82 \\
\hline 20939 & Sva & 0.99 & 0.81 & 0.84 \\
\hline 20944 & Svs5 & 1.01 & 0.78 & 0.82 \\
\hline 232811 & Suv420h2 & 0.98 & 0.61 & 0.73 \\
\hline 237336 & Tbpl1 & 0.98 & 0.66 & 0.67 \\
\hline 27381 & Tcl1b2 & 0.99 & 0.65 & 1.02 \\
\hline 387347 & Tas2r118 & 0.97 & 0.83 & 0.98 \\
\hline 387515 & Tas2r144 & 0.96 & 0.67 & 0.70 \\
\hline 53878 & Svs2 & 1.01 & 0.83 & 0.92 \\
\hline 57252 & Tas2r105 & 0.96 & 0.74 & 0.89 \\
\hline 574417 & Tas2r137 & 0.97 & 0.65 & 0.71 \\
\hline 67043 & Syap1 & 0.97 & 0.63 & 0.65 \\
\hline 67923 & Tceb1 & 0.98 & 0.82 & 1.17 \\
\hline 68416 & Sycn & 0.98 & 0.70 & 0.78 \\
\hline 71578 & Sval1 & 0.97 & 0.68 & 0.76 \\
\hline 93670 & Tac4 & 0.96 & 0.77 & 0.83 \\
\hline 104871 & Spata7 & 1.03 & 0.64 & 0.64 \\
\hline 20759 & Sprr2e & 0.99 & 0.77 & 0.89 \\
\hline 20760 & Sprr2f & 0.96 & 0.88 & 1.04 \\
\hline 20761 & Sprr2g & 1.02 & 0.75 & 0.79 \\
\hline 20770 & Spt1 & 0.99 & 0.85 & 0.95 \\
\hline 225888 & Suv420h1 & 1.04 & 0.84 & 1.00 \\
\hline 229285 & Spg20 & 0.96 & 0.65 & 0.74 \\
\hline 278240 & Spin2 & 0.96 & 0.79 & 0.87 \\
\hline 54402 & Stk19 & 0.97 & 0.51 & 0.56 \\
\hline 68720 & Lce1b & 0.98 & 0.87 & 0.98 \\
\hline 69611 & Lce1d & 1.02 & 0.70 & 0.73 \\
\hline 70599 & Ssfa2 & 1.02 & 0.96 & 1.07 \\
\hline 75956 & Srrm2 & 0.98 & 0.67 & 0.76 \\
\hline 216395 & Tmem5 & 1.02 & 0.89 & 0.96 \\
\hline 21667 & Tdgf1 & 1.09 & 0.91 & 1.03 \\
\hline 219249 & Tdrd3 & 1.02 & 0.76 & 0.88 \\
\hline 27380 & Tcl1b4 & 1.00 & 0.88 & 1.04 \\
\hline 27382 & Tcl1b5 & 1.09 & 0.76 & 0.76 \\
\hline 56351 & Tebp & 1.02 & 0.85 & 0.95 \\
\hline 67878 & Tmem33 & 1.00 & 0.84 & 0.99 \\
\hline 73122 & Tgfbrap1 & 1.02 & 0.69 & 0.98 \\
\hline 73679 & Tex19.1 & 1.01 & 0.81 & 0.84 \\
\hline 83559 & Tex18 & 1.04 & 0.64 & 0.66 \\
\hline 108946 & Zzz3 & 1.04 & 0.76 & 0.88 \\
\hline 112405 & Egln1 & 1.04 & 0.95 & 1.04 \\
\hline 112406 & Egln2 & 0.99 & 0.88 & 1.21 \\
\hline 14204 & $\| 4 \mathrm{i} 1$ & 1.00 & 0.93 & 1.17 \\
\hline 15495 & Hsd3b4 & 1.05 & 0.63 & 0.77 \\
\hline 15496 & Hsd3b5 & 0.98 & 0.57 & 0.73 \\
\hline 170737 & Znrf1 & 1.02 & 0.76 & 1.10 \\
\hline 20250 & Scd2 & 1.01 & 0.79 & 0.79 \\
\hline 20834 & Znrf4 & 1.00 & 0.70 & 0.87 \\
\hline 22761 & Zfpm1 & 1.05 & 0.68 & 0.82 \\
\hline 22789 & Zp3r & 1.01 & 0.79 & 0.89 \\
\hline
\end{tabular}




\begin{tabular}{|c|c|c|c|c|}
\hline 319740 & Zfyve27 & 1.04 & 0.95 & 0.97 \\
\hline 320951 & 9030221M09Rik & 1.01 & 0.61 & 0.63 \\
\hline 52696 & Zwint & 1.05 & 0.61 & 0.81 \\
\hline 58865 & Tdh & 1.01 & 0.67 & 0.86 \\
\hline 68221 & 1700049M11Rik & 0.99 & 0.74 & 0.91 \\
\hline 68842 & Tulp4 & 1.03 & 0.87 & 1.29 \\
\hline 69367 & Glrx2 & 1.01 & 0.74 & 0.86 \\
\hline 74551 & 1810010014Rik & 0.99 & 0.90 & 1.01 \\
\hline 77424 & 9530002K18Rik & 0.98 & 0.76 & 0.82 \\
\hline 78287 & Zfyve20 & 1.00 & 0.89 & 0.95 \\
\hline 113865 & V1rc8 & 1.03 & 0.88 & 0.88 \\
\hline 171245 & V1rh1 & 1.06 & 0.82 & 0.94 \\
\hline 171247 & Vmn1r199 & 1.01 & 0.73 & 0.78 \\
\hline 171257 & V1ri6 & 1.04 & 0.75 & 0.87 \\
\hline 171271 & Vmn1r220 & 1.05 & 0.81 & 0.88 \\
\hline 213081 & Wdr19 & 1.01 & 0.73 & 0.90 \\
\hline 22360 & Vmp & 1.04 & 0.84 & 0.92 \\
\hline 230796 & Wdtc1 & 1.02 & 0.71 & 0.74 \\
\hline 252908 & V1ri8 & 1.04 & 0.76 & 0.78 \\
\hline 27973 & Vkorc1 & 1.03 & 0.77 & 0.79 \\
\hline 54636 & Wdr45 & 1.03 & 0.73 & 0.74 \\
\hline 69568 & Vkorc1l1 & 1.01 & 0.64 & 0.65 \\
\hline 113848 & Vmn1r42 & 0.99 & 0.71 & 0.87 \\
\hline 113864 & V1rc7 & 1.03 & 0.82 & 0.99 \\
\hline 171198 & Vmn1r28 & 0.98 & 0.76 & 0.88 \\
\hline 212190 & Ubxd3 & 1.04 & 0.68 & 0.83 \\
\hline 217379 & Ubxd4 & 1.00 & 0.71 & 0.99 \\
\hline 22269 & Upk2 & 1.02 & 0.61 & 0.81 \\
\hline 22270 & Upk3a & 0.99 & 0.80 & 0.86 \\
\hline 69136 & Tusc1 & 1.06 & 0.69 & 0.90 \\
\hline 70450 & Unc13d & 1.01 & 0.89 & 1.24 \\
\hline 72565 & Uaca & 1.03 & 0.62 & 0.63 \\
\hline 80385 & Tusc2 & 1.05 & 0.89 & 1.23 \\
\hline 215627 & Zbtb8 & 1.08 & 0.76 & 0.79 \\
\hline 22631 & Ywhaz & 1.06 & 0.77 & 0.79 \\
\hline 54367 & Zfp326 & 1.10 & 0.90 & 1.27 \\
\hline 231659 & G431004K08Rik & 1.09 & 0.88 & 1.03 \\
\hline 67230 & 2810439M05Rik & 1.08 & 0.73 & 0.84 \\
\hline 12189 & Brca1 & 1.02 & 0.93 & 1.28 \\
\hline 208104 & B930074I24 & 1.00 & 0.94 & 1.28 \\
\hline 216874 & Camta2 & 1.01 & 0.94 & 0.99 \\
\hline 232855 & BC023179 & 1.00 & 1.04 & 1.30 \\
\hline 235048 & BC050092 & 1.01 & 0.90 & 1.05 \\
\hline 235956 & Zfp825 & 1.00 & 0.88 & 1.00 \\
\hline 244219 & BC030314 & 1.01 & 1.09 & 1.13 \\
\hline 319792 & 9130023H24Rik & 1.03 & 0.84 & 1.15 \\
\hline 381066 & Zfp948 & 1.01 & 0.84 & 1.13 \\
\hline 66830 & Nacc1 & 1.01 & 0.97 & 1.21 \\
\hline 78783 & Brpf1 & 1.02 & 0.93 & 1.03 \\
\hline 406222 & Krt74 & 1.03 & 0.93 & 1.01 \\
\hline 71994 & Cnn3 & 1.03 & 0.89 & 1.20 \\
\hline 102162 & Taf5l & 1.06 & 0.86 & 1.05 \\
\hline 74469 & Taf7l & 1.02 & 0.99 & 1.06 \\
\hline 328280 & Rslcan24 & 1.05 & 1.02 & 1.10 \\
\hline
\end{tabular}




\begin{tabular}{|c|c|c|c|c|}
\hline 20288 & Msr1 & 1.14 & 0.79 & 0.81 \\
\hline 239029 & 4933430J11 & 1.21 & 0.65 & 0.65 \\
\hline 18171 & Nr1i2 & 1.14 & 0.68 & 0.72 \\
\hline 257891 & Olfr479 & 1.14 & 0.54 & 0.61 \\
\hline 258016 & Olfr453 & 1.14 & 0.62 & 0.77 \\
\hline 258222 & Olfr310 & 1.19 & 0.71 & 0.75 \\
\hline 258270 & Olfr448 & 1.11 & 0.60 & 0.62 \\
\hline 258293 & Olfr437 & 1.10 & 0.74 & 0.77 \\
\hline 258302 & Olfr420 & 1.14 & 0.79 & 0.80 \\
\hline 258436 & Olfr458 & 1.12 & 0.61 & 1.01 \\
\hline 258490 & Olfr492 & 1.19 & 0.43 & 0.43 \\
\hline 258529 & Olfr313 & 1.15 & 0.56 & 0.57 \\
\hline 258530 & Olfr311 & 1.16 & 0.60 & 0.70 \\
\hline 258648 & Olfr237-ps1 & 1.18 & 0.68 & 0.71 \\
\hline 258713 & Olfr430 & 1.14 & 0.61 & 0.61 \\
\hline 258926 & Olfr476 & 1.13 & 0.69 & 0.72 \\
\hline 257908 & Olfr115 & 1.21 & 0.84 & 1.01 \\
\hline 257899 & Olfr1000 & 1.20 & 0.71 & 0.79 \\
\hline 257916 & Olfr1031 & 1.16 & 0.66 & 0.67 \\
\hline 18442 & P2ry2 & 1.13 & 0.72 & 0.78 \\
\hline 259112 & Olfr979 & 1.12 & 0.50 & 0.53 \\
\hline 102607 & Snx19 & 1.11 & 0.77 & 0.78 \\
\hline 14700 & Gng10 & 1.07 & 0.98 & 1.11 \\
\hline 19385 & Ranbp1 & 1.16 & 0.51 & 0.56 \\
\hline 19387 & Rangap1 & 1.15 & 0.40 & 0.45 \\
\hline 20403 & Itsn2 & 1.11 & 0.82 & 1.09 \\
\hline 227800 & Rabgap1 & 1.20 & 0.48 & 0.51 \\
\hline 236573 & E430029F06 & 1.09 & 0.86 & 1.00 \\
\hline 243362 & Stard13 & 1.07 & 0.79 & 0.94 \\
\hline 244962 & Snx14 & 1.24 & 0.69 & 0.75 \\
\hline 380711 & Garnl4 & 1.08 & 0.83 & 0.83 \\
\hline 50780 & Rgs3 & 1.14 & 0.82 & 0.87 \\
\hline 56395 & C78915 & 1.12 & 0.79 & 0.95 \\
\hline 56784 & Ralgapa1 & 1.09 & 0.82 & 1.00 \\
\hline 70497 & Arhgap17 & 1.11 & 0.79 & 0.91 \\
\hline 73910 & Arhgap18 & 1.15 & 0.80 & 0.93 \\
\hline 76117 & Arhgap15 & 1.13 & 0.85 & 0.88 \\
\hline 79264 & Krit1 & 1.07 & 0.76 & 0.84 \\
\hline 99326 & Garnl3 & 1.13 & 0.71 & 0.72 \\
\hline 107435 & Hat1 & 1.07 & 0.78 & 1.33 \\
\hline 216454 & BC089597 & 1.04 & 0.78 & 0.93 \\
\hline 77805 & A930014I12Rik & 1.06 & 0.88 & 1.04 \\
\hline 114143 & Atp6v0b & 1.05 & 0.87 & 1.00 \\
\hline 232906 & 6430596G11Rik & 1.05 & 0.80 & 0.81 \\
\hline 68449 & 1110003P22Rik & 1.05 & 0.84 & 0.93 \\
\hline 17110 & Lzp-s & 1.06 & 0.29 & 0.53 \\
\hline 17766 & Nudt1 & 1.05 & 0.90 & 0.97 \\
\hline 18010 & Neu1 & 1.07 & 0.98 & 1.11 \\
\hline 207806 & Gm608 & 1.07 & 0.92 & 1.17 \\
\hline 212442 & Lactb2 & 1.06 & 0.35 & 0.65 \\
\hline 215951 & Lace1 & 1.08 & 0.33 & 0.58 \\
\hline 223254 & Farp1 & 1.05 & 0.85 & 1.06 \\
\hline 226090 & MGC28180 & 1.08 & 0.79 & 1.10 \\
\hline 227377 & Farp2 & 1.06 & 0.91 & 0.99 \\
\hline
\end{tabular}




\begin{tabular}{|c|c|c|c|c|}
\hline 227619 & Man1b1 & 1.05 & 0.38 & 0.63 \\
\hline 23956 & Neu2 & 1.07 & 0.94 & 1.09 \\
\hline 268395 & Mpg & 1.05 & 0.91 & 1.16 \\
\hline 30959 & $\mathrm{Ddx} 25$ & 1.07 & 0.89 & 1.09 \\
\hline 320685 & Dctd & 1.09 & 0.77 & 0.80 \\
\hline 52633 & Nit2 & 1.07 & 0.78 & 0.89 \\
\hline 59007 & Ngly1 & 1.09 & 0.94 & 1.06 \\
\hline 67328 & Lyzl1 & 1.07 & 0.38 & 0.66 \\
\hline 71567 & Mcmdc1 & 1.09 & 0.90 & 1.13 \\
\hline 72831 & Dhx30 & 1.05 & 0.81 & 0.93 \\
\hline 80907 & Lactb & 1.05 & 0.36 & 0.64 \\
\hline 83456 & Mov10l1 & 1.08 & 0.90 & 1.02 \\
\hline 101497 & Al194308 & 1.06 & 0.97 & 1.24 \\
\hline 102075 & 4931414L13Rik & 1.04 & 0.92 & 1.00 \\
\hline 107338 & Gbf1 & 1.04 & 1.01 & 1.34 \\
\hline 109151 & Chd9 & 1.03 & 0.92 & 1.02 \\
\hline 11938 & Atp2a2 & 1.04 & 0.89 & 1.04 \\
\hline 11941 & Atp2b2 & 1.05 & 0.92 & 1.13 \\
\hline 11981 & Atp9a & 1.11 & 1.13 & 1.31 \\
\hline 16801 & Arhgef1 & 1.03 & 1.00 & 1.22 \\
\hline 209224 & Cova1 & 1.07 & 0.93 & 1.03 \\
\hline 213484 & BC036718 & 1.04 & 0.82 & 0.89 \\
\hline 213522 & BC026778 & 1.03 & 0.32 & 0.63 \\
\hline 216848 & Chd3 & 1.02 & 0.81 & 1.02 \\
\hline 217364 & D230014K01Rik & 1.04 & 0.82 & 0.88 \\
\hline 224079 & 9330174J19Rik & 1.11 & 1.03 & 1.10 \\
\hline 227723 & 5830434P21Rik & 1.04 & 0.83 & 0.84 \\
\hline 229688 & BC051070 & 1.03 & 0.92 & 0.94 \\
\hline 230379 & Acer2 & 1.07 & 0.86 & 0.94 \\
\hline 243272 & BC021875 & 1.03 & 0.46 & 0.73 \\
\hline 319767 & 9030605H24Rik & 1.06 & 1.04 & 1.10 \\
\hline 320024 & Aadacl1 & 1.02 & 0.52 & 0.57 \\
\hline 320707 & Atp2b3 & 1.02 & 0.93 & 1.11 \\
\hline 381572 & 9430007A20Rik & 1.06 & 0.87 & 1.01 \\
\hline 50769 & Atp8a2 & 1.06 & 1.01 & 1.07 \\
\hline 52666 & D10Ertd610e & 1.08 & 0.93 & 0.96 \\
\hline 53404 & Atoh7 & 1.06 & 1.10 & 1.50 \\
\hline 54608 & Abhd2 & 1.06 & 0.94 & 0.97 \\
\hline 67239 & Bxdc1 & 1.03 & 0.97 & 1.13 \\
\hline 67772 & Chd8 & 1.05 & 0.90 & 1.01 \\
\hline 68126 & Fahd2a & 1.02 & 0.63 & 0.89 \\
\hline 71562 & Afmid & 1.02 & 0.95 & 1.28 \\
\hline 74018 & Als2 & 1.04 & 1.08 & 1.14 \\
\hline 80744 & BC003993 & 1.02 & 0.54 & 0.71 \\
\hline 83379 & AV071179 & 1.04 & 0.97 & 1.27 \\
\hline 21843 & Tial1 & 1.04 & 0.78 & 0.81 \\
\hline 219131 & Phf11 & 1.05 & 0.70 & 0.71 \\
\hline 547168 & Rhox7 & 1.07 & 0.90 & 0.96 \\
\hline 57317 & Sfrs4 & 1.05 & 0.87 & 1.12 \\
\hline 68927 & Ptcd2 & 1.04 & 0.91 & 0.96 \\
\hline 100609 & 9830109N13Rik & 1.05 & 0.90 & 1.02 \\
\hline 103284 & Zc3h10 & 1.02 & 1.13 & 1.23 \\
\hline 12877 & Cpeb1 & 1.04 & 1.20 & 1.39 \\
\hline 13831 & Epc1 & 1.04 & 1.01 & 1.06 \\
\hline
\end{tabular}




\begin{tabular}{|c|c|c|c|c|}
\hline 14359 & Fxr1h & 1.02 & 0.92 & 0.94 \\
\hline 226049 & Dmrt2 & 1.01 & 1.13 & 1.34 \\
\hline 231044 & Gbx1 & 1.03 & 0.98 & 1.13 \\
\hline 231999 & Plekha8 & 1.01 & 0.89 & 1.18 \\
\hline 233904 & BC010250 & 1.02 & 1.15 & 1.17 \\
\hline 242620 & Dmrta2 & 1.01 & 1.10 & 1.22 \\
\hline 268741 & 5730589K01Rik & 1.03 & 0.74 & 0.80 \\
\hline 268859 & A2bp1 & 1.06 & 1.02 & 1.33 \\
\hline 320858 & D930040M24Rik & 1.02 & 0.89 & 1.06 \\
\hline 494448 & Cbx6 & 1.03 & 0.87 & 1.09 \\
\hline 66408 & Aptx & 1.06 & 1.16 & 1.36 \\
\hline 70127 & Dpf3 & 1.02 & 0.97 & 1.14 \\
\hline 217944 & Rapgef5 & 1.06 & 0.84 & 0.90 \\
\hline 26464 & Vnn3 & 1.07 & 1.02 & 1.27 \\
\hline 67229 & Prpf18 & 1.06 & 0.97 & 1.02 \\
\hline 67724 & Pop1 & 1.07 & 0.78 & 0.86 \\
\hline 72886 & 2900016D05Rik & 1.06 & 0.94 & 1.11 \\
\hline 74002 & Psd2 & 1.07 & 0.94 & 0.97 \\
\hline 14957 & Hist1h1d & 1.05 & 1.12 & 1.17 \\
\hline 14958 & $\mathrm{H} 1 \mathrm{fO}$ & 1.04 & 0.93 & 0.98 \\
\hline 15078 & H3f3a & 1.02 & 0.92 & 1.06 \\
\hline 15267 & Hist2h2aa1 & 1.07 & 1.10 & 1.28 \\
\hline 15371 & $\mathrm{Hm} \times 1$ & 1.07 & 1.23 & 1.75 \\
\hline 15372 & $\mathrm{Hm} \times 2$ & 1.03 & 1.08 & 1.40 \\
\hline 16815 & Lbx2h & 1.04 & 0.98 & 1.13 \\
\hline 18080 & Nin & 1.02 & 0.89 & 1.02 \\
\hline 18508 & Pax6 & 1.06 & 1.11 & 1.29 \\
\hline 223499 & Gm83 & 1.04 & 0.88 & 0.91 \\
\hline 228731 & Nkx2-4 & 1.04 & 1.07 & 1.31 \\
\hline 234988 & Mbd3I2 & 1.01 & 1.13 & 1.45 \\
\hline 237339 & L3mbtl3 & 1.02 & 1.20 & 1.29 \\
\hline 243529 & $\mathrm{H} 1 \mathrm{fx}$ & 1.03 & 0.99 & 0.99 \\
\hline 246738 & ORF28 & 1.04 & 0.93 & 1.02 \\
\hline 260423 & Hist1h3f & 1.05 & 1.12 & 1.41 \\
\hline 27354 & $\mathrm{Nbn}$ & 1.02 & 0.44 & 0.78 \\
\hline 319158 & Hist1h4i & 1.05 & 1.10 & 1.31 \\
\hline 382035 & Pabpn1I & 1.07 & 1.03 & 1.09 \\
\hline 433762 & LOC433762 & 1.05 & 1.04 & 1.41 \\
\hline 50708 & Hist1h1c & 1.05 & 1.17 & 1.39 \\
\hline 56335 & Mett|3 & 1.03 & 0.42 & 0.69 \\
\hline 56702 & Hist1h1b & 1.02 & 1.13 & 1.17 \\
\hline 80838 & Hist1h1a & 1.03 & 1.02 & 1.18 \\
\hline 97908 & Hist1h3g & 1.03 & 1.08 & 1.16 \\
\hline 30928 & Zfp238 & 1.06 & 0.75 & 0.89 \\
\hline 67876 & 1500041J02Rik & 1.07 & 0.77 & 0.83 \\
\hline 69029 & 1500032L24Rik & 1.02 & 0.79 & 0.99 \\
\hline 69770 & 1600002K03Rik & 1.07 & 0.66 & 0.78 \\
\hline 75188 & 1700009J07Rik & 1.03 & 0.75 & 0.98 \\
\hline 75462 & 1700001C19Rik & 1.02 & 0.72 & 0.95 \\
\hline 76568 & 1500035H01Rik & 1.05 & 0.82 & 1.04 \\
\hline 78304 & 1500034E06Rik & 1.02 & 0.78 & 0.90 \\
\hline 78330 & 1500032D16Rik & 1.02 & 0.80 & 0.88 \\
\hline 66183 & 1110032A04Rik & 1.07 & 0.79 & 0.90 \\
\hline 66195 & 1110058A15Rik & 1.07 & 0.82 & 0.87 \\
\hline
\end{tabular}




\begin{tabular}{|c|c|c|c|c|}
\hline 68659 & 1110032E23Rik & 1.08 & 0.87 & 0.99 \\
\hline 68731 & Rbfa & 1.09 & 0.74 & 0.88 \\
\hline 68796 & 1110039B18Rik & 1.07 & 0.58 & 0.61 \\
\hline 73730 & 1110008K04Rik & 1.08 & 0.86 & 0.97 \\
\hline 74165 & 1110004B15Rik & 1.12 & 0.84 & 1.00 \\
\hline 381629 & 0610007C21Rik & 1.00 & 0.72 & 1.01 \\
\hline 55978 & Ift20 & 1.02 & 0.72 & 1.05 \\
\hline 56700 & 0610031J06Rik & 1.03 & 0.70 & 0.80 \\
\hline 57247 & Zfp276 & 1.03 & 0.74 & 1.04 \\
\hline 59052 & Mett19 & 1.00 & 0.75 & 1.06 \\
\hline 66048 & 0610009E20Rik & 1.00 & 0.78 & 0.96 \\
\hline 66074 & 0610041E09Rik & 1.02 & 0.85 & 0.93 \\
\hline 66089 & Rmnd5b & 1.03 & 0.68 & 0.96 \\
\hline 66910 & Tmem107 & 1.06 & 0.66 & 0.78 \\
\hline 67441 & 0610042E07Rik & 1.01 & 0.89 & 0.98 \\
\hline 68212 & 0610007H07Rik & 1.02 & 0.67 & 0.84 \\
\hline 68314 & 0610008F07Rik & 0.99 & 0.80 & 0.88 \\
\hline 68323 & 0610006K04Rik & 1.02 & 0.84 & 0.88 \\
\hline 68421 & 0910001K20Rik & 1.02 & 0.86 & 0.98 \\
\hline 68550 & 1110002 N22Rik & 1.00 & 0.86 & 0.99 \\
\hline 71691 & Pnmal1 & 1.01 & 0.81 & 1.03 \\
\hline 77038 & Arfgap2 & 1.05 & 0.80 & 0.91 \\
\hline 109082 & 1110064L07Rik & 1.13 & 0.79 & 1.02 \\
\hline 231238 & 2310045A20Rik & 1.06 & 0.82 & 1.00 \\
\hline 65971 & Tbata & 1.07 & 0.87 & 0.96 \\
\hline 66374 & 2310011J03Rik & 1.06 & 0.88 & 0.94 \\
\hline 71897 & 2310010M24Rik & 1.08 & 0.91 & 0.94 \\
\hline 66934 & 1700022L09Rik & 1.06 & 0.88 & 1.02 \\
\hline 67317 & 1700022I11Rik & 1.08 & 0.90 & 1.02 \\
\hline 69479 & 1700029J07Rik & 1.06 & 0.99 & 1.43 \\
\hline 73274 & 1700034P14Rik & 1.05 & 0.89 & 1.08 \\
\hline 73299 & 1700041G16Rik & 1.05 & 0.93 & 1.26 \\
\hline 75564 & 1700027N10Rik & 1.09 & 0.83 & 1.14 \\
\hline 76416 & Znrd1as & 1.08 & 0.93 & 0.96 \\
\hline 108735 & 2010005013Rik & 1.15 & 0.83 & 0.96 \\
\hline 109129 & 2010311D03Rik & 1.12 & 0.73 & 0.82 \\
\hline 233545 & 2210018M11Rik & 1.08 & 0.84 & 0.87 \\
\hline 53951 & 2310002B06Rik & 1.11 & 0.88 & 0.90 \\
\hline 56786 & Tmem9b & 1.14 & 0.90 & 0.94 \\
\hline 66343 & Tmem177 & 1.12 & 0.89 & 1.01 \\
\hline 66349 & 2310004L02Rik & 1.08 & 0.85 & 1.06 \\
\hline 66353 & 2310007A19Rik & 1.17 & 0.99 & 1.00 \\
\hline 66358 & 2310004I24Rik & 1.16 & 0.65 & 0.71 \\
\hline 66359 & Fam36a & 1.08 & 0.95 & 0.96 \\
\hline 66488 & Fam136a & 1.10 & 0.80 & 0.88 \\
\hline 66526 & 2210012G02Rik & 1.04 & 0.88 & 0.94 \\
\hline 66528 & 2210020M01Rik & 1.16 & 1.05 & 1.10 \\
\hline 66549 & Aggf1 & 1.08 & 0.80 & 0.87 \\
\hline 67017 & 2010011I20Rik & 1.17 & 0.97 & 1.16 \\
\hline 67038 & 2010109I03Rik & 1.11 & 0.86 & 0.96 \\
\hline 67298 & Gprasp1 & 1.11 & 0.81 & 0.92 \\
\hline 67484 & Eepd1 & 1.13 & 0.99 & 1.02 \\
\hline 67495 & Tmem167b & 1.11 & 0.90 & 0.97 \\
\hline 67620 & 2310006J04Rik & 1.10 & 0.90 & 0.95 \\
\hline
\end{tabular}




\begin{tabular}{|c|c|c|c|c|}
\hline 67899 & $\mathrm{Cmc1}$ & 1.10 & 0.90 & 1.02 \\
\hline 69147 & 2200002J24Rik & 1.08 & 0.89 & 0.93 \\
\hline 69457 & 2310005G13Rik & 1.10 & 0.94 & 0.96 \\
\hline 69520 & Lce3f & 1.12 & 0.80 & 0.90 \\
\hline 69542 & 2300002M23Rik & 1.06 & 0.84 & 0.94 \\
\hline 69871 & 2010007H12Rik & 1.08 & 0.88 & 1.09 \\
\hline 69876 & 2010013E08Rik & 1.14 & 0.98 & 1.08 \\
\hline 69882 & 2010321M09Rik & 1.10 & 0.93 & 1.15 \\
\hline 69893 & 2010305A19Rik & 1.12 & 0.99 & 1.00 \\
\hline 69894 & 2010107G23Rik & 1.19 & 0.79 & 0.96 \\
\hline 70082 & 2210402C18Rik & 1.18 & 0.91 & 1.05 \\
\hline 70088 & 2310005N01Rik & 1.14 & 0.87 & 0.93 \\
\hline 70160 & Vps36 & 1.14 & 0.86 & 0.97 \\
\hline 70163 & 2210415F13Rik & 1.09 & 0.73 & 0.73 \\
\hline 70178 & 2210412D01Rik & 1.18 & 0.87 & 0.97 \\
\hline 70257 & 2010107E04Rik & 1.06 & 0.89 & 0.96 \\
\hline 71886 & 2310002L09Rik & 1.14 & 0.99 & 1.01 \\
\hline 71912 & 2300003C06Rik & 1.15 & 0.81 & 0.95 \\
\hline 72084 & 2010319C14Rik & 1.10 & 0.97 & 1.10 \\
\hline 72098 & 2010300G19Rik & 1.05 & 0.93 & 0.97 \\
\hline 72103 & 2010301N04Rik & 1.13 & 0.87 & 0.87 \\
\hline 72123 & 2010109K11Rik & 1.14 & 0.94 & 1.01 \\
\hline 72357 & 2210016L21Rik & 1.05 & 1.04 & 1.07 \\
\hline 74175 & 2300002G24Rik & 1.10 & 0.90 & 0.95 \\
\hline 74243 & 2210009G21Rik & 1.12 & 0.88 & 0.95 \\
\hline 75691 & Anks6 & 1.09 & 0.98 & 1.13 \\
\hline 76425 & Gid8 & 1.04 & 1.07 & 1.09 \\
\hline 76527 & 2010004A03Rik & 1.11 & 1.04 & 1.05 \\
\hline 98682 & 2210010L05Rik & 1.16 & 0.68 & 0.71 \\
\hline 67291 & 3110023B02Rik & 1.24 & 0.88 & 0.92 \\
\hline 108755 & 2610208E05Rik & 1.12 & 1.03 & 1.25 \\
\hline 108909 & 2610208M17Rik & 1.16 & 0.84 & 1.10 \\
\hline 214459 & 2610318I01Rik & 1.19 & 0.93 & 1.24 \\
\hline 54614 & 2610317D23Rik & 1.15 & 1.10 & 1.47 \\
\hline 66459 & 2610022G08Rik & 1.15 & 0.87 & 1.09 \\
\hline 66460 & 2610042014Rik & 1.16 & 0.98 & 1.23 \\
\hline 67171 & Dram2 & 1.18 & 0.92 & 1.07 \\
\hline 68032 & 2610318K02Rik & 1.15 & 0.94 & 1.18 \\
\hline 69900 & Mfsd11 & 1.14 & 0.85 & 1.12 \\
\hline 70297 & Gcc2 & 1.17 & 0.83 & 0.94 \\
\hline 70333 & Cd3eap & 1.13 & 1.00 & 1.16 \\
\hline 70420 & 2610034B18Rik & 1.20 & 1.03 & 1.31 \\
\hline 70466 & Ckap2l & 1.19 & 0.95 & 1.18 \\
\hline 72137 & Wdsub1 & 1.17 & 0.93 & 1.14 \\
\hline 72139 & 2610044015Rik & 1.14 & 1.00 & 1.25 \\
\hline 72141 & Adpgk & 1.12 & 0.86 & 1.13 \\
\hline 72155 & 2610510J17Rik & 1.13 & 1.04 & 1.24 \\
\hline 72201 & 2600013N14Rik & 1.16 & 0.99 & 1.23 \\
\hline 72503 & 2610507B11Rik & 1.13 & 0.78 & 0.98 \\
\hline 72519 & 2610319K07Rik & 1.12 & 1.01 & 1.12 \\
\hline 76375 & 2610034H20Rik & 1.12 & 0.81 & 1.07 \\
\hline 76915 & 2610034E18Rik & 1.19 & 1.02 & 1.17 \\
\hline 66985 & 2400009B11Rik & 1.17 & 0.90 & 0.98 \\
\hline 108900 & 2700049P18Rik & 1.14 & 0.99 & 1.17 \\
\hline
\end{tabular}




\begin{tabular}{|c|c|c|c|c|}
\hline 230582 & Cyb5rl & 1.08 & 0.95 & 1.23 \\
\hline 269633 & 2810046M22Rik & 1.11 & 1.01 & 1.09 \\
\hline 66310 & 2810410M20Rik & 1.09 & 0.90 & 1.32 \\
\hline 67217 & 2810055F11Rik & 1.10 & 0.95 & 1.02 \\
\hline 68020 & 2810002N01Rik & 1.12 & 0.93 & 1.26 \\
\hline 68026 & 2810417H13Rik & 1.12 & 0.70 & 0.83 \\
\hline 70211 & 2810407A14Rik & 1.12 & 0.92 & 1.08 \\
\hline 72174 & 2810423G08Rik & 1.18 & 1.05 & 1.19 \\
\hline 72195 & Supt7I & 1.07 & 0.89 & 1.11 \\
\hline 72522 & 2610528J18Rik & 1.11 & 0.66 & 0.69 \\
\hline 72543 & 2610528K11Rik & 1.10 & 0.67 & 0.77 \\
\hline 72649 & Tmem209 & 1.10 & 0.83 & 1.02 \\
\hline 72669 & 2810032G03Rik & 1.07 & 0.82 & 1.05 \\
\hline 78833 & 2700085M18Rik & 1.08 & 0.95 & 1.15 \\
\hline 67745 & 4930583K01Rik & 1.10 & 0.82 & 1.11 \\
\hline 73167 & 3110043J09Rik & 1.14 & 0.82 & 1.01 \\
\hline 74383 & Ubap2l & 1.11 & 0.64 & 0.68 \\
\hline 99887 & 4930577M16Rik & 1.10 & 0.85 & 1.00 \\
\hline 67574 & 4833435D08Rik & 1.13 & 0.66 & 0.76 \\
\hline 78757 & 4921505C17Rik & 1.15 & 0.86 & 1.05 \\
\hline 225392 & Rell2 & 1.08 & 0.95 & 1.16 \\
\hline 233826 & 4732427B05 & 1.11 & 0.99 & 1.14 \\
\hline 245843 & 4632417D23 & 1.09 & 0.60 & 0.64 \\
\hline 66701 & 4633402N23Rik & 1.10 & 0.82 & 1.13 \\
\hline 69823 & 3830421F13Rik & 1.06 & 0.88 & 1.09 \\
\hline 76863 & 4833420K19Rik & 1.07 & 1.02 & 1.09 \\
\hline 77043 & 4632433K11Rik & 1.06 & 0.80 & 1.06 \\
\hline 77056 & Tmco4 & 1.06 & 0.98 & 1.27 \\
\hline 67592 & 4930524B15Rik & 1.17 & 0.75 & 0.89 \\
\hline 75283 & 4930556L07Rik & 1.16 & 0.66 & 0.77 \\
\hline 109212 & 6720460F02Rik & 1.20 & 1.06 & 1.09 \\
\hline 71406 & 5430416009Rik & 1.15 & 0.92 & 1.00 \\
\hline 108654 & 4933403F05Rik & 1.11 & 0.91 & 1.04 \\
\hline 244178 & 4933400K24Rik & 1.10 & 0.84 & 0.99 \\
\hline 381622 & 5031410I06Rik & 1.17 & 0.99 & 1.14 \\
\hline 66775 & 4933428I03Rik & 1.12 & 1.01 & 1.06 \\
\hline 71323 & 5133400D11Rik & 1.14 & 0.85 & 0.95 \\
\hline 75991 & 5033405K12Rik & 1.11 & 0.77 & 0.91 \\
\hline 244886 & Al118078 & 1.16 & 0.91 & 1.09 \\
\hline 64074 & Smoc2 & 1.07 & 0.75 & 0.82 \\
\hline 216724 & Rufy1 & 1.08 & 0.54 & 0.56 \\
\hline 83564 & Rnh2 & 1.08 & 0.73 & 0.83 \\
\hline 19328 & Rab12 & 1.07 & 0.84 & 0.85 \\
\hline 19362 & Rad51ap1 & 1.09 & 0.61 & 0.62 \\
\hline 245195 & Retnlg & 1.04 & 0.67 & 0.73 \\
\hline 245688 & Rbbp7 & 1.05 & 0.90 & 1.18 \\
\hline 69903 & Rasip1 & 1.08 & 0.63 & 0.70 \\
\hline 70052 & Prpf4 & 1.06 & 0.92 & 0.98 \\
\hline 76938 & Rbm17 & 1.05 & 0.56 & 0.56 \\
\hline 24056 & Sh3bp5 & 1.09 & 0.73 & 0.78 \\
\hline 27387 & Sh2d3c & 1.07 & 0.86 & 0.89 \\
\hline 68723 & S100a18 & 1.08 & 0.75 & 0.77 \\
\hline 20409 & Ostf1 & 1.08 & 0.59 & 0.63 \\
\hline 242737 & Oog4 & 1.05 & 0.74 & 0.86 \\
\hline
\end{tabular}




\begin{tabular}{|l|l|l|l|l|}
258225 & Olfr913 & 1.05 & 0.86 & 1.14 \\
\hline 258500 & Olfr944 & 1.05 & 0.61 & 0.75 \\
\hline 68767 & Wash & 1.05 & 0.59 & 0.63 \\
\hline 257898 & Olfr867 & 1.10 & 0.68 & 0.71 \\
\hline
\end{tabular}


Table 5-2. PANTHER Identified receptor/receptor activity hits.

\begin{tabular}{|c|c|c|c|c|c|}
\hline \multirow{2}{*}{$\begin{array}{l}\text { Entrez } \\
\text { Gene } \\
\text { ID }\end{array}$} & \multirow{2}{*}{$\begin{array}{l}\text { Gene } \\
\text { Symbol }\end{array}$} & \multirow{2}{*}{ Gene Name } & \multicolumn{3}{|c|}{ Screen Survival } \\
\hline & & & $20 \mathrm{~min}$ & 2.0hr & $10.0 \mathrm{hr}$ \\
\hline 11539 & Adora1 & Adenosine receptor $\mathrm{A} 1$ & 1.17 & 0.74 & 0.74 \\
\hline 12766 & Cxcr3 & C-X-C chemokine receptor type 3 & 1.08 & 1.01 & 1.30 \\
\hline 12801 & Cnr1 & Cannabinoid receptor 1 & 1.10 & 0.93 & 1.03 \\
\hline 12813 & Col10a1 & Collagen alpha- $1(X)$ chain & 1.07 & 0.82 & 0.88 \\
\hline 13051 & Cx3cr1 & CX3C chemokine receptor 1 & 1.16 & 0.94 & 1.03 \\
\hline 13488 & Drd1a & $\mathrm{D}(1 \mathrm{~A})$ dopamine receptor & 1.13 & 1.15 & 1.23 \\
\hline 13490 & Drd3 & $\mathrm{D}(3)$ dopamine receptor & 1.16 & 1.08 & 1.20 \\
\hline 13491 & Drd4 & $\mathrm{D}(4)$ dopamine receptor & 1.16 & 0.85 & 1.09 \\
\hline 13609 & Edg1 & Sphingosine 1-phosphate receptor 1 & 1.17 & 0.99 & 1.30 \\
\hline 13617 & Ednra & Endothelin-1 receptor & 1.13 & 0.65 & 0.70 \\
\hline 14062 & $\mathrm{~F} 2 \mathrm{r}$ & Proteinase-activated receptor 1 & 1.15 & 0.96 & 1.22 \\
\hline 14065 & $\mathrm{~F} 2 \mathrm{rl3}$ & Proteinase-activated receptor 4 & 1.14 & 1.04 & 1.28 \\
\hline 14293 & Fpr1 & fMet-Leu-Phe receptor & 1.30 & 1.23 & 1.39 \\
\hline 14294 & Fprl1 & Formyl peptide receptor-related sequence 1 & 1.12 & 1.00 & 1.19 \\
\hline 14527 & Gcgr & Glucagon receptor & 1.18 & 0.78 & 0.82 \\
\hline 14602 & Ghrhr & Growth hormone-releasing hormone receptor & 1.09 & 0.94 & 0.98 \\
\hline 14652 & Glp1r & Glucagon-like peptide 1 receptor & 1.11 & 0.82 & 0.85 \\
\hline 14715 & Gnrhr & Gonadotropin-releasing hormone receptor & 1.15 & 0.95 & 1.03 \\
\hline 14744 & Gpr65 & Psychosine receptor & 1.12 & 0.84 & 0.90 \\
\hline 14747 & Cmklr1 & Chemokine-like receptor 1 & 1.16 & 0.87 & 1.04 \\
\hline 15370 & $\mathrm{Nr} 4 \mathrm{a} 1$ & Nuclear receptor subfamily 4 group A member 1 & 1.06 & 0.63 & 0.77 \\
\hline 15551 & $\mathrm{Htr} 1 \mathrm{~b}$ & 5-hydroxytryptamine receptor $1 \mathrm{~B}$ & 1.12 & 0.94 & 1.14 \\
\hline 16639 & Klra8 & Killer cell lectin-like receptor 8 & 1.08 & 1.08 & 1.09 \\
\hline 16995 & Ltb4r1 & Leukotriene B4 receptor 1 & 1.10 & 0.88 & 1.02 \\
\hline 18171 & Nr1i2 & Nuclear receptor subfamily 1 group I member 2 & 1.14 & 0.68 & 0.72 \\
\hline 18227 & $\mathrm{Nr} 4 \mathrm{a} 2$ & Nuclear receptor subfamily 4 group A member 2 & 1.07 & 1.06 & 1.60 \\
\hline 18441 & P2ry1 & P2Y purinoceptor 1 & 1.10 & 0.45 & 0.52 \\
\hline 18442 & P2ry2 & P2Y purinoceptor 2 & 1.13 & 0.72 & 0.78 \\
\hline 19214 & Ptgdr & Prostaglandin D2 receptor & 1.13 & 0.71 & 0.75 \\
\hline 19220 & Ptgfr & Prostaglandin F2-alpha receptor & 1.06 & 0.48 & 0.59 \\
\hline 19222 & Ptgir & Prostacyclin receptor & 1.01 & 0.80 & 1.15 \\
\hline 19228 & Pthr1 & $\begin{array}{l}\text { Parathyroid hormone/parathyroid hormone-related } \\
\text { peptide receptor }\end{array}$ & 1.03 & 0.99 & 1.39 \\
\hline 19401 & Rara & Retinoic acid receptor alpha & 1.09 & 0.86 & 0.91 \\
\hline 20288 & Msr1 & Macrophage scavenger receptor types I and II & 1.14 & 0.79 & 0.81 \\
\hline 20605 & Sstr1 & Somatostatin receptor type 1 & 1.03 & 0.76 & 0.87 \\
\hline
\end{tabular}




\begin{tabular}{|c|c|c|c|c|c|}
\hline 20607 & Sstr3 & Somatostatin receptor type 3 & 1.05 & 1.04 & 1.13 \\
\hline 20608 & Sstr4 & Somatostatin receptor type 4 & 1.06 & 0.68 & 0.96 \\
\hline 20609 & Sstr5 & Somatostatin receptor type 5 & 1.00 & 0.78 & 1.22 \\
\hline 21337 & Tacr2 & Substance-K receptor & 1.01 & 0.86 & 1.03 \\
\hline 21338 & Tacr3 & Neuromedin-K receptor & 1.05 & 0.63 & 0.68 \\
\hline 21833 & Thra & Thyroid hormone receptor alpha & 1.22 & 0.87 & 1.01 \\
\hline 21834 & Thrb & Thyroid hormone receptor beta & 0.99 & 0.92 & 1.16 \\
\hline 21907 & Nr2e1 & Nuclear receptor subfamily 2 group E member 1 & 1.06 & 0.44 & 0.50 \\
\hline 21926 & Tnf & Tumor necrosis factor & 1.09 & 0.52 & 0.54 \\
\hline 22045 & Trhr & Thyrotropin-releasing hormone receptor & 1.00 & 1.10 & 1.56 \\
\hline 22337 & Vdr & Vitamin D3 receptor & 1.11 & 0.59 & 0.60 \\
\hline 22354 & Vipr1 & Vasoactive intestinal polypeptide receptor 1 & 1.04 & 0.95 & 1.32 \\
\hline 22355 & Vipr2 & Vasoactive intestinal polypeptide receptor 2 & 1.03 & 0.76 & 0.95 \\
\hline 23957 & $\mathrm{NrOb2}$ & Nuclear receptor subfamily 0 group B member 2 & 1.06 & 1.01 & 1.41 \\
\hline 23958 & $\mathrm{Nr2e3}$ & Photoreceptor-specific nuclear receptor & 1.10 & 0.49 & 0.58 \\
\hline 50908 & C1s & Complement C1s-A subcomponent & 1.08 & 0.86 & 0.91 \\
\hline 50928 & Klrg1 & Killer cell lectin-like receptor subfamily G member 1 & 1.09 & 0.85 & 0.86 \\
\hline 52389 & $\begin{array}{l}\text { D7Ertd68 } \\
\text { Oe }\end{array}$ & MCG21623, isoform CRA_b & 1.16 & 0.96 & 1.29 \\
\hline 52614 & Emr4 & $\begin{array}{l}\text { EGF-like module-containing mucin-like hormone } \\
\text { receptor-like } 4\end{array}$ & 1.11 & 1.06 & 1.34 \\
\hline 54160 & Copg2 & Granzyme A & 1.00 & 0.99 & 1.36 \\
\hline 56544 & V2R2 & Vomeronasal type-2 receptor 1 & 1.05 & 0.77 & 1.13 \\
\hline 56696 & Gpr132 & Probable G-protein coupled receptor 132 & 1.09 & 0.96 & 1.21 \\
\hline 57385 & P2ry4 & P2Y purinoceptor 4 & 1.02 & 0.78 & 1.12 \\
\hline 64095 & Gpr35 & G-protein coupled receptor 35 & 1.11 & 0.68 & 0.69 \\
\hline 67168 & $\mathrm{P} 2 \mathrm{y} 5$ & Lysophosphatidic acid receptor 6 & 1.06 & 0.80 & 0.89 \\
\hline 68897 & Disp1 & Protein dispatched homolog 1 & 1.10 & 1.06 & 1.07 \\
\hline 70839 & P2ry12 & P2Y purinoceptor 12 & 1.01 & 0.86 & 1.02 \\
\hline 71326 & Treml1 & Trem-like transcript 1 protein & 1.00 & 0.70 & 0.83 \\
\hline 71991 & Ckn1 & DNA excision repair protein ERCC-8 & 1.07 & 0.93 & 1.00 \\
\hline 78560 & Gpr124 & G-protein coupled receptor 124 & 1.28 & 1.10 & 1.10 \\
\hline 80910 & Gpr84 & G-protein coupled receptor 84 & 1.17 & 0.97 & 1.06 \\
\hline 84111 & Gpr87 & G-protein coupled receptor 87 & 1.09 & 0.87 & 0.96 \\
\hline 84112 & Sucnr1 & Succinate receptor 1 & 1.02 & 0.88 & 1.23 \\
\hline 94109 & Csmd1 & CUB and sushi domain-containing protein 1 & 1.04 & 1.05 & 1.05 \\
\hline 94226 & Edg8 & Sphingosine 1-phosphate receptor 5 & 1.18 & 1.19 & 1.53 \\
\hline 97998 & Deptor & Rod outer segment membrane protein 1 & 1.06 & 1.08 & 1.10 \\
\hline 101533 & $\begin{array}{l}1200016 \\
\text { C12Rik }\end{array}$ & Kallikrein 1-related peptidase b9 & 1.06 & 0.94 & 1.01 \\
\hline 170757 & Eltd1 & $\begin{array}{l}\text { EGF, latrophilin seven transmembrane domain- } \\
\text { containing protein } 1\end{array}$ & 1.23 & 0.81 & 0.87 \\
\hline
\end{tabular}




\begin{tabular}{|c|c|c|c|c|c|}
\hline 213527 & Pthr2 & Parathyroid hormone 2 receptor & 1.06 & 1.05 & 1.80 \\
\hline 216749 & Nmur2 & Neuromedin-U receptor 2 & 1.10 & 0.84 & 0.85 \\
\hline 226278 & Prlhr & Prolactin-releasing peptide receptor & 1.12 & 1.06 & 1.51 \\
\hline 227288 & II8ra & C-X-C chemokine receptor type 1 & 1.10 & 0.77 & 0.90 \\
\hline 229323 & Gpr171 & Probable G-protein coupled receptor 171 & 1.14 & 0.78 & 0.93 \\
\hline 233571 & P2ry6 & P2Y purinoceptor 6 & 1.02 & 0.82 & 1.08 \\
\hline 238252 & Gpr135 & Probable G-protein coupled receptor 135 & 1.17 & 0.75 & 0.79 \\
\hline 239029 & $\begin{array}{l}4933430 \mathrm{~J} \\
11\end{array}$ & Anthrax toxin receptor-like & 1.21 & 0.65 & 0.65 \\
\hline 239853 & Gpr128 & Probable G-protein coupled receptor 128 & 1.17 & 0.85 & 0.85 \\
\hline 243083 & $\begin{array}{l}\text { Tmprss11 } \\
\mathrm{f}\end{array}$ & Transmembrane protease serine $11 \mathrm{~F}$ & 1.11 & 0.84 & 1.06 \\
\hline 243084 & $\begin{array}{l}\text { A030012 } \\
\text { E10 }\end{array}$ & Transmembrane protease serine $11 \mathrm{E}$ & 1.03 & 0.89 & 1.27 \\
\hline 243277 & $\begin{array}{l}\text { E230012 } \\
\text { M21 }\end{array}$ & Probable G-protein coupled receptor 133 & 1.16 & 0.99 & 1.42 \\
\hline 245688 & Rbbp7 & Histone-binding protein RBBP7 & 1.05 & 0.90 & 1.18 \\
\hline 257891 & Olfr479 & Olfactory receptor Olfr479 & 1.14 & 0.54 & 0.61 \\
\hline 257898 & Olfr867 & Olfactory receptor 867 & 1.10 & 0.68 & 0.71 \\
\hline 258302 & Olfr420 & Olfactory receptor & 1.14 & 0.79 & 0.80 \\
\hline 258713 & Olfr430 & Olfactory receptor & 1.14 & 0.61 & 0.61 \\
\hline 258925 & Olfr20 & Olfactory receptor & 1.07 & 0.76 & 0.81 \\
\hline 269053 & Gpr152 & Probable G-protein coupled receptor 152 & 1.16 & 0.90 & 0.98 \\
\hline 321019 & Ebi2 & G-protein coupled receptor 183 & 1.14 & 0.78 & 0.85 \\
\hline 387285 & Hertr2 & Orexin receptor type 2 & 1.15 & 0.92 & 1.00 \\
\hline 435845 & $\begin{array}{l}\text { Tmprss11 } \\
\mathrm{C}\end{array}$ & Neurobin & 1.17 & 0.73 & 0.74 \\
\hline 436440 & Gpr31c & $\begin{array}{l}\text { 12-(S)-hydroxy-5,8,10,14-eicosatetraenoic } \\
\text { receptor }\end{array}$ & 1.14 & 0.79 & 0.83 \\
\hline
\end{tabular}


CHAPTER 6:

DISCUSSION AND FUTURE DIRECTIONS 


\section{$\underline{\text { Research Summary }}$}

I demonstrated that $Y$. pestis survival within the macrophage is a dynamic process and several host proteins are directly involved in YCV biogenesis. My data, summarized in Fig. 6-1, demonstrates that within 20 mins post-infection $Y$. pestis prevents lysosome fusion through recruitment of Rab1b and Rab4a to arrest YCV acidification. By 80 mins post-infection the maturing YCV associates with Rab1b, Rab4a and Rab11b. In addition to recruitment of Rab proteins involved in endocytic recycling to the YCV, Y. pestis infection also stalls the host recycling process, and this is necessary for late stage bacterial replication. My data suggests that stalling of the recycling process can be directly attributed to sequestering Rab11b on the YCV, as overexpression of Rab11b restores recycling and prevents bacterial replication. These data provide a significant improvement in our understanding of the early colonization of the macrophage and trafficking events leading the maturation of the YCV into a replicative niche.

\section{Implications of My Research and Future Directions}

\section{Developing new pathway analysis techniques for RNAi screens through mapping interactions}

Our identification and enrichment for host cell trafficking processes and ultimately the impact of the endocytic recycling pathway on $Y$. pestis survival was due in part to our novel approach to genomic screening data. To the best of my knowledge, to date all RNAi genomic screens have focused their post-screen efforts using pathway and ontology analysis software to understand their validated data [130-142], and not to explore their datasets in a biological context during validation. As such, we initially used MetaCore pathway analysis software to determine enrichment within our validated gene hits. However, due to the limited number of input genes (135), we were unable to identify specific pathways with any degree of confidence. In many instances, the significantly enriched networks only contained a single gene that skewed the $p$-value due to the weight of the gene within the identified network. Whereas another networks would contain multiple genes, but the combined p-value was not sufficient to warrant significance by the pathway analysis software. Part of this is the nature of RNAi genomic screens versus microarray analysis, which 
pathway analysis programs were developed for. RNAi does not have $100 \%$ penetrance, meaning the targeted gene is usually only inhibited to an undefined degree. In contrast, a microarray has $100 \%$ penetrance, meaning the impact upon the phenotype of interest can be completely dictated by the experimental design (e.g., gene deletion or growth condition). As such, enrichment for a pathway based on gene p-value is difficult to interpret in the context of an RNAi screen. Furthermore, statistical weight is assigned to each cellular component based on their interconnectedness with other cellular proteins. As a result, transcriptional and global pathway regulators have larger statistical weight when included in the user's dataset. As such, a dataset could be significantly skewed by the number of transcriptional or global regulators within the dataset, resulting in analysis missing important pathways. Therefore, we shifted our bioinformatic analysis to Gene Ontology (GO) clustering to generate and build maps of interacting gene partners based on our validated and primary screen hits. These initial interaction maps then served to guide our subsequent experiments.

Curated pathways are also limited to the quality and known knowledge of the genes within the pathways. This limitation ultimately skews screen analysis toward what is known or predicted. Thus, we chose to build maps of interacting partners within our validated and primary screen hits to avoid any bias inherent to current analysis software. This allowed our networks to self-enrich through inclusion of additional nodes that strengthened the interactions between input datasets. Moving forward, one could use this process to further understand the relationship between various subsets identified in our validated dataset. Also one could use this process to understand bacterial invasion through mapping invasion hits to our survival hits. Furthermore, we can begin development of an application using these simple rules to allow fellow pathway explorers to build maps of their datasets.

\section{Are Rab1b and Rab4a only required to avoid YCV acidification?}

Y. pestis recruits Rab1b and Rab4a within 20 mins post-infection of the macrophage to prevent YCV acidification (chapters 3 and 4;[61]). These findings show $Y$. pestis survival within the macrophage hinges on early subversion of phagolysosome maturation. However, it is unclear if Rab1b and Rab4a are required only to prevent YCV acidification or if they are also essential for $Y$. 
pestis YCV biogenesis/survival post-phagolysosome subversion. To address this, one could use chemical inhibitors, such as ammonium chloride, to block vacuole acidification in Rab1b and Rab4a siRNA treated macrophages and then monitor survival of $Y$. pestis. If either of these Rabs are required to manipulate the macrophages in subsequent steps after preventing YCV acidification, Y. pestis bacterial survival will still be inhibited in RNAi treated cells. In contrast, if these Rabs are strictly recruited to block acidification, ammonium chloride treatment should restore bacterial survival in RNAi treated cells. In the event treatment does not restore virulence, further characterization of the YCV biogenesis process in these cells will need to be performed.

\section{Does the Timing of Rab Recruitment Impact YCV Maturation?}

Rab GTPase trafficking occurs in coordinated and sequential fashion to efficiently move the vesicle to its intended destination along an endocytic pathway within the cell (see reviews [102, 185, 289, 290]). For example, Rabs 5, 7 and 9 are recruited in a specific sequence to achieve phagosome fusion with the lysosome [94, 95, 98-101]. Disrupting the recruitment of one protein results in failure to recruit subsequent Rabs, terminating phagolysosome maturation [94, 95, 98101]. We have shown $Y$. pestis quickly subverts/arrests phagolysosome maturation and prevents YCV acidification within 20 mins of entry into the macrophage by recruiting Rab1b and Rab4a (chapter 3 and 4; [61]). However, we have not determined if recruitment of either of these proteins is required for subsequent recruitment of Rab11b. Using RNAi to inhibit Rab1b and Rab4a, one could determine if Rab11b is still recruited to the YCV in the absence of these other Rab proteins. These experiments can be further extended to identify if loss of any of the other validated Rab proteins impact recruitment. Moreover, future studies using CRISPR/CAS systems to integrate multiple fluorescently tagged Rabs $1 \mathrm{~b}, 4 \mathrm{a} \& 11 \mathrm{~b}$ and live cell imaging could provide a powerful tool to monitor Rab GTPase timing and bacterial trafficking in real-time. Importantly, live cell imaging could also incorporate tagged markers for cellular processes such as autophagy, receptors, and/or the phagolysosome to allow for real-time analysis of: 1) Y. pestis trafficking Rab interactions that lead to autophagy, recycling endosome maturation and phagolysosome avoidance, 2) Y. pestis time and spatial dependent recruitment of Rabs and host markers to support autophagy, recycling endosome maturation and phagolysosome avoidance 3) Y. pestis time dependent modeling of 
trafficking and replication requirements during YCV maturation, and 4) identification and data to develop computational systems to demonstrate/animate Rab GTPase mediated trafficking events in silico. Understanding the timing of Rab GTPase recruitment events may also reveal if one Rab protein is targeted by $Y$. pestis to influence the recruitment of others, resulting in prioritizing potential Rab proteins for subsequent studies to understand how $Y$. pestis recruits these proteins to the YCV.

\section{How does Y. pestis recruit Rab GTPases to the YCV?}

Efforts to identify $Y$. pestis proteins responsible for intracellular survival by targeting the bacterium itself using mutagenic screens have had limited success, and have only identified the PhoP/Q regulon as required [86, 291-293]. We have identified nine Rab GTPases that appear to be required for $Y$. pestis survival (Rabs 1b, 2b, 3d, 4a, 19, 20, 23, 30, \& 40b). Based on their cellular location and similarity of GTP binding domains, these Rabs can be classified into five subfamilies: Subclass 1: Rab23; Subclass 9: Rab20; Subclass 11: Rab2b, Rab4a; Subclass 12: Rab19, Rab30; or Subclass 13: Rab1b, Rab3d, Rab40b [294-297]. Our enrichment for Rab GTPases involved with host endocytic recycling suggests $Y$. pestis makes a bacterial effector that recognizes a specific motif within these Rab GTPases [294-297]. Using Rab1b, Rab4a and Rab11b as "bait", immunoprecipitation could be performed to pull down $Y$. pestis proteins that potential interact with these Rabs. The bacterial proteins would then be identified by MS/MS. Mutations in these genes could then be made and tested for Rab recruitment, intracellular survival, and virulence in the mouse model. It should be noted that $Y$. pestis may indirectly recruit Rab proteins through interactions with host Rab effectors. In this scenario, $Y$. pestis proteins may not precipitate via Rab pull down. In the event that no proteins are identified via pull downs, transposon mutagenesis could be used to identify $Y$. pestis mutants that do not recruit Rabs to the early YCV (using high content screening). Host targets of these $Y$. pestis proteins could then be identified by immunoprecipitation of eukaryotic lysates. 
How do Y. pestis interactions with the host cell recycling pathway contribute to intracellular survival?

Cross talk between endocytic recycling and autophagy is a potential cellular mechanism for sensing disruptions within trafficking pathways or inadequate homeostatic signals from receptors involved in maintaining cellular metabolism [298]. Furthermore, recent studies have indicated that Rab11b may be an important mediator of this cross talk [254]. Y. pestis has been shown to associate with autophagosomes; however, the contribution of autophagy to $Y$. pestis replication is yet to be determined $[59,88,89]$. During the first $8 \mathrm{~h}$ of infection, $Y$. pestis induces autophagy within infected cells, as indicated by the conversion of LC3-I to LC3-II [59, 299]. Pujol et al. showed that $Y$. pestis avoids clearance by xenophagy, resides in autophagosomes, but does not require autophagy to survive in macrophages up to $8 \mathrm{~h}$ post infection [59]. Studies with $Y$. pseudotuberculosis, the closest relative to $Y$. pestis, have shown autophagy is required for bacterial replication [88, 89]. Additionally, Moreau et al. have shown that induction of autophagy with rapamycin increased metabolic activity of $Y$. pseudotuberculosis within the YCV, and abolishing autophagy induction using Atg4b C74A (enzymatically inactive Atg4b) prevented the recruitment of LC3 to the YCV [88]. Therefore, it is reasonable to hypothesize that by stalling the host recycling pathway, Y. pestis may disrupt metabolic signals resulting in the host cell, becoming quiescent and inducing autophagic flux in the cell. This hypothesis could explain how Y. pestis induces autophagy. To test if autophagy is specifically required for bacterial replication independent of host cell recycling, one could treat $Y$. pestis infected Rab11b overexpressing macrophages with rapamycin to chemically induce autophagy independent of recycling inhibition. If autophagy is required for $Y$. pestis replication, induction of autophagy using rapamycin in $Y$. pestis infected Rab11b overexpressing cells would restore bacterial replication to wildtype levels. However, it is possible that autophagy is not required for $Y$. pestis replication/survival. In this case induction of autophagy will not restore bacterial replication in Rab11b overexpressing cells. Furthermore, these results would suggest bacterial replication is dependent on an unknown recycling endosome maturation event, and induction of autophagy within infected cells is a byproduct of the infection. 
Extending our Rab11b studies suggests a universal mechanism for intracellular pathogens

In addition to $Y$. pestis, a variety of other pathogens appear to target Rab11 (a/b) [107, 130, $131,140-142,191,194,215-217,240,246,248,300-312]$. Anthrax toxins were shown to disrupt recycling in Drosophila wing discs, and overexpression of Rab11 restored wildtype morphology $[248,313]$. This theme has been observed for Tetanus and Shiga toxin B through their disruption and association with markers for recycling endosomes [240, 309, 310]. While these toxins are secreted from their respected pathogens, neither Bacillus anthracis, Shigella flexneri or Clostridium tetani appear to reside in a pathogen containing vacuole (PCV) [103, 314]. However, the targeting of Rab11 by intracellular pathogens indicates a potentially universal mechanism supporting survival (summary in Table 6-1). Future studies determining if these pathogens target Rab11 to disrupt host cellular recycling processes to promote survival need to be conducted. Perhaps diverting/stalling Rab11 naturally driven processes through the dilution/sequestration/inactivation of Rab GTPase protein available to the cell would allow these organisms to establish their replicative niches, by modulating downstream innate cellular defenses, such as autophagy $[254,315]$.

\section{Does stalling of host recycling potentially impact pro-inflammatory responses?}

Y. pestis infection produces very little pro-inflammatory response early during infection, but at latter stages there is a dramatic spike in inflammation [1, 316-322]. This phenomena is also observed in transcritpome analysis of monocytes/ macrophages infected with $Y$. pestis lacking the pCD1 virulence plasmid and T3SS secreted Yops [321], which are potent modulators of the inflammasome and pro-inflammatory apoptosis [323-325]. Rab11 trafficking has also been shown to impact many host proteins involved in innate immunity. For example, efficient signaling and recycling of TLR4 requires Rab11a and CD14 [71, 326, 327]. Moreover, it is known that Rab11 is required for both loading/storing $\mathrm{MHC}$ I within recycling endosomes, but also for recruitment of MHC I to endosomes engaged in active TLR4 signaling [328, 329]. Finally, Weigert et al. found dominant negative forms of Rab11 and Rab22a prevented MHC I recycling [330]. One can view these findings as two separate but integral events for TLR4 and MHC I signaling as mediated by Rab11 trafficking processes: 1) pre-priming recycling endocytic compartments with MHC I and recycling/trafficking MHC I to the plasma membrane, and 2) TLR4 engaged signaling promoting 
recruitment of MHC I via Rab11 to initiate an innate immune response (perhaps autophagy/xenophagy). Due to the requirement for Rab11 in TLR4 and MHC I cellular processes, one can speculate that $Y$. pestis sequestration of Rab11b may also disrupting TLR4 and MHC I signaling cascades. This disruption could potentially contribute to anti-inflammatory nature of $Y$. pestis infection.

To determine if TLR4 signaling is disrupted, one could adapt our TfR recycling assay to monitor TLR4 recycling and TLR4 retention in macrophages infected with $Y$. pestis and compare to infection with E. coli K12 or S. Typhimurium. Given our previous findings that TfR is retained due to disruption of host recycling, we expect to see retention of TLR4 in Y. pestis infected cells and inefficient recycling of TLR4. These results would suggest that $Y$. pestis infected macrophages may be further inhibited in their ability to respond to LPS through TLR4 signaling. To determine if MHC I presentation is disrupted, one could pulse $Y$. pestis infected macrophages with Ova SIINFEKL peptide. Using flow cytometry, one can compare MHC I expression of $Y$. pestis infected cells to uninfected cells. If $Y$. pestis disrupts MHC I presentation, one would expect to see diminished surface expression of MHC I specifically in $Y$. pestis infected cells. Furthermore, classical T-cell activation assays could be used to determine if MHC antigen presentation is disrupted by $Y$. pestis infection.

An extension of these studies would be to adoptively transfer $Y$. pestis infected wildtype and Rab11b overexpressing macrophages into mice. Herein, one could determine if stalling cellular host recycling by $Y$. pestis contributes to: 1) dampening the early pro-inflammatory response of infected hosts via cytokine response, 2) dissemination of $Y$. pestis, or 3) overall animal survival. The results of these studies will provide biological significance for stalling host cell recycling in $Y$. pestis infected macrophages within the context of in vivo pathogenesis of plague. Moreover, these adoptive macrophage studies could have novel insight into the overall contribution of $Y$. pestis intracellular survival within the macrophage in terms of disease progression. Overall, these studies would be the first to determine if $Y$. pestis disruption of host endocytic recycling to the dampening of pro-inflammatory response of an infected host. 


\section{Does $Y$. pestis use eukaryotic receptors to target the recycling pathway?}

The first step of any pathogen to colonize a host cell is adherence and invasion. We identified 96 potential receptors that exhibit a $Y$. pestis invasion phenotype from our RNAi genome-wide screen. Of the 96 identified receptors exhibiting a $Y$. pestis invasion phenotype, we hit Edg1 (Sphingosine 1-phosphate receptor 1). This receptor has been shown to inhibit bacterial dissemination from the draining lymph node in vivo [49]. Furthermore, St. John et al. demonstrated that chemical ablation of Edg1 reduced $Y$. pestis burden in CD11b (macrophage) and CD11C (dendritic) cell types in the popliteal and iliac lymph nodes [49]. Due to these previous findings, Edg1 should be prioritized for further characterization using experiments detailed in chapter 5.

Interestingly, recycling endosomes are hallmarked by very little acidification, and remain at $\mathrm{pH} 6.4$ to prevent degradation of vacuole contents in order to recycle these reusable cellular components [244, 331]. Therefore, the ability of $Y$. pestis to inhibit YCV acidification could be directly linked to the receptor it uses for invasion, if this receptor targets the endosome to the recycling pathway. Several chemokine and scavenger receptors were identified as potential $Y$. pestis receptors. These receptors have been shown to associate with recycling endosomes, and potentially have common motifs or interacting partners [332, 333]. Using our validated screen hits as a focal point, one could map network interactions to the 96 receptors to determine if a common pathway emerges. In support of this, we identified Arfgap2, an interacting partner of Arf1, within our invasion dataset [288]. Arf1 interacts with Rab4a to facilitate recycling endosome trafficking from the early endosome [238, 244, 334]. These findings indicate that $Y$. pestis entry and/or early survival may directly link to the pathogen's ability to traffic into the host recycling pathway.

It is possible that $Y$. pestis uses multiple receptors for invasion, or binds to a cluster of receptors associated within a lipid raft. In this case, there may be several receptors that inhibit $Y$. pestis invasion, but the efficiency of each individual receptor to inhibit invasion would matter. As such, one could use chemical inhibitors to further determine the general mechanism for internalization of $Y$. pestis. Overall, identifying a receptor that prevents uptake of $Y$. pestis is advantageous. Once identified, one could define the bacterial proteins that facilitate this through 
screening a transposon library or using bacterial outer membrane preparations in pull-down assays with tagged versions of the receptors. Through the identification of these bacterial proteins one could generate deletion mutations of $Y$. pestis and assess the contribution invasion has on pathogenesis. Importantly, these bacterial proteins could serve as potential targets to develop new therapeutics or vaccines to inhibit macrophage invasion.

\section{Is spatial location within the cell important for intracellular survival?}

Using live cell microscopy, we have observed dynamic YCV movement occurring during the first 8 hours of infection (unpublished data). We have also observed that $Y$. pestis tends to replicate only in YCVs that traffic to the perinuclear region (unpublished data). In contrast, bacteria that remain spatially halfway between the plasma membrane and perinuclear region appear to be degraded, presumably within phagolysosomes. Recently, Johnson et al. have shown that heterogeneity exists in lysosomes within the cell [335]. Their results demonstrate the further from the plasma membrane the more efficient the acidification process is through accumulation (increased density) of vATPase and Rab7 [335]. Intriguingly, this data suggests that spatial and temporal areas of the cell are more capable of mounting innate immune responses than others. Looking at cellular location and similarity of GTP binding domains of the nine validated Rabs (Rabs 1b, 2b, 3d, 4a, 19, 20, 23, 30, \& 40b) we noticed enrichment for the Golgi/ ERGIC perinuclear space, indicating that recruitment of these Rabs to the YCV may result in trafficking towards the Golgi/ ERGIC space. These observations suggest that $Y$. pestis survival and avoidance of the phagolysosome may be directly linked to trafficking of the bacteria to the perinuclear region. Using markers for lysosomes (vATPase, Rab7, Rab9 \& Cathepsin D) and image analysis software, one could determine the spatial requirements for $Y$. pestis survival/replication. Moreover, using RNAi techniques and markers for the Golgi/ERGIC space, one could determine trafficking events and spatial location requirements for $Y$. pestis replication. If temporal and spatial location are essential for Y. pestis replication/survival, we would expect to see differences in recruitment of Rab GTPases and phagolysosome markers to perinuclear YCVs in contrast to their counterparts closer to the plasma membrane. 


\section{Potential Biological Impact of Y. pestis Low Calcium Response in Macrophages}

Y. pestis has a growth phenomenon termed the "Low Calcium Response" (LCR) discovered in the late $1940-50$ s $[1,75,82,336,337]$. The LCR is characterized as a biological requirement for $\mathrm{Ca}^{2+}$ for growth at $37^{\circ} \mathrm{C}$ in broth media, and no supplementation for growth at $26^{\circ} \mathrm{C}$ (see review [1]). Furthermore, consecutive passages at $37^{\circ} \mathrm{C}$ in the absence of $\mathrm{Ca}^{2+}$ results in loss of the pCD1 plasmid, which encodes the T3SS, and attenuation in mice [336, 337]. To date, there is little known about the biological relevance for the LCR [1, 338-341]. Szatmari and colleagues recently demonstrated that Rab11b interacts with Hook, a negative regulator of endosome maturation, to facilitate crosstalk between recycling endosomes and induction of autophagy [254]. Furthermore, autophagy triggers an influx of calcium into the nascent autophagic vesicle, and Rab11b is required for the effective fusion and docking during this transition [342, 343]. These observations suggests that $Y$. pestis induction of autophagy may result in the induction of $\mathrm{Ca}^{2+}$ influx into the YCV and subsequently trigger bacterial replication. To determine if calcium influx occurs in the YCV, cells could be infected with fluorescent $Y$. pestis strain and pulsed with a calcium fluro dye to monitor the changes in $\mathrm{YCV} \mathrm{Ca}{ }^{2+}$ concentration over time. Simultaneously one could determine when the LCR is induced during a macrophage infection by monitoring the expression levels of ICrF (a gene whose expression changes during the LCR) during macrophage infection using a bioluminescent transcriptional reporter. If /crF expression is dependent on $\mathrm{Ca}^{2+}$ influx, then we would expect induction of IcrF expression to occur after influx of calcium into the YCV. In the event that $\mathrm{Ca}^{2+}$ influx and $/ c r F$ expression changes are observed, one could then determine if Rab11b recruitment prevents the induction of LCR. In either case, these experiments would clearly demonstrate a biological role for LCR of $Y$. pestis in bacterial replication during bacterial survival within the macrophage. In addition to growth at $37^{\circ} \mathrm{C}$, Y. pestis secretion of the T3SS Yops effector proteins is also regulated by calcium and the IcrF (see review [344]). If indeed LCR responses are triggered with $\mathrm{Ca}^{2+}$ influx into the YCV, one could also determine if $Y$. pestis T3SS expression is also triggered by $\mathrm{Ca}^{2+}$ influx. If expression is induced during intracellular infection, studies to determine if secretion of the Yop effectors occurs in response to $\mathrm{Ca}^{2+}$ influx could be performed. 
These studies would provide a novel insight into how $Y$. pestis regulates mammalian specific virulence factors, and senses the transition into the mammalian host. This would also suggest that $Y$. pestis modulation of mammalian expressed virulence factors is not solely dependent on temperature shift. Findings may explain why in vitro growth of $Y$. pestis require calcium. This also suggests that spontaneous loss of pCD1 in naturally occurring environmental isolates is a response of $Y$. pestis adapting to an environment with little potential for transmission to a mammalian host. Furthermore, to our knowledge there is not a calcium driven immune response to $Y$. pestis within the flea midgut. Potentially, the influence of a sustained calcium event and $a 37^{\circ} \mathrm{C}$ temperature shift is the necessary trigger for $Y$. pestis to sense intracellular survival in a mammal vs extracellular survival within the flea. 
Figures and Figure Legends

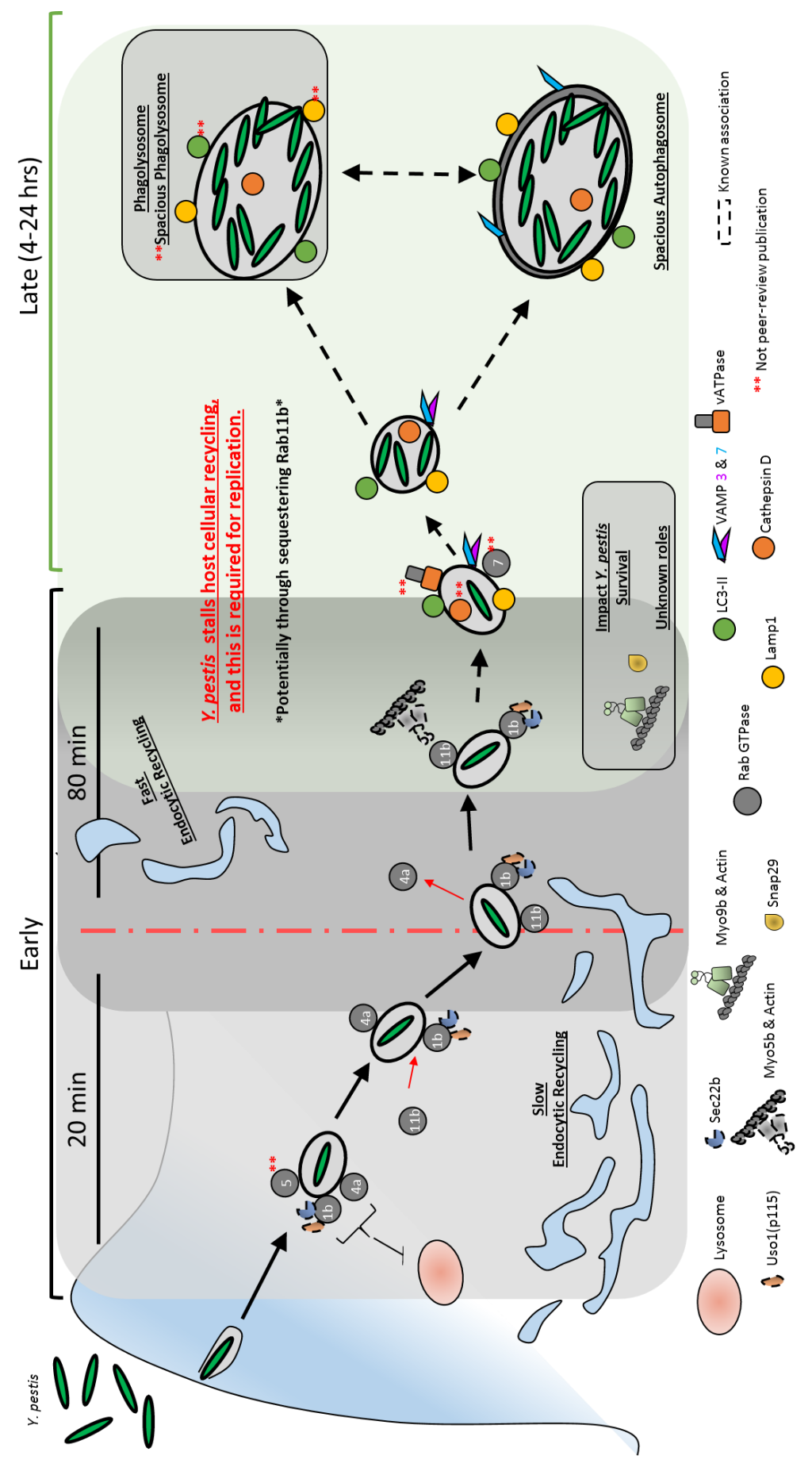

Figure 6-1. Summary model of our findings 
$\underline{\text { Tables }}$

Table 6-1. Rab11 (a/b) pathogen \& toxin association.

\begin{tabular}{|c|c|c|}
\hline \multicolumn{3}{|c|}{ Rab11 (a/b) Pathogen \& Toxin Association } \\
\hline Pathogen & $\begin{array}{l}\text { Rab11 }(\mathrm{a} / \mathrm{b}) \text { loss impact on } \\
\text { replication/survival? }\end{array}$ & Ref. \\
\hline C. burnetii & Small vacuoles & {$[131,215,217,300-302]$} \\
\hline L.. pneumophila & ?; is on the LCV & [304] \\
\hline L. monocytogenes & Inhibits & {$[130,141]$} \\
\hline S. aureus & ?; has dynamic co-localization & [108] \\
\hline S. flexneri & Inhibits vacuole rupture & {$[305,306]$} \\
\hline S. typhimurium & $\begin{array}{l}\text { Partially inhibits; required for efficient } \\
\text { maturation }\end{array}$ & [307] \\
\hline Mycobacterium sp & Inhibits?; cell type specificity? & {$[142,311]$} \\
\hline Anaplasma sp & Inhibits & {$[191,216]$} \\
\hline Chlamydia sp & Inhibits & {$[140,194,246]$} \\
\hline Andes virus & Inhibits & [312] \\
\hline Hantavirus (new world) & Inhibits & [312] \\
\hline Respiratory syncytial virus & $?$ & [303] \\
\hline Toxin & $\begin{array}{c}\text { Rab11 (a/b) overexpression rescue } \\
\text { phenotype? }\end{array}$ & Ref. \\
\hline Anthrax (EF \& LF) & Yes, for EF toxin only & [248] \\
\hline Cholera & Yes & [308] \\
\hline Tetanus & Yes & {$[240,309]$} \\
\hline Shiga B & Yes & [310] \\
\hline
\end{tabular}




\section{REFERENCES}

1. Perry, R.D. and J.D. Fetherston, Yersinia pestis--etiologic agent of plague. Clin Microbiol Rev, 1997. 10(1): p. 35-66.

2. $\quad$ Langer, W.L., THE BLACK DEATH. Sci Am, 1964. 210: p. 114-21.

3. Konkola, K., More than a coincidence? The arrival of arsenic and the disappearance of plaque in early modern Europe. J Hist Med Allied Sci, 1992. 47(2): p. 186-209.

4. CDC - Maps \& Statistics - Plague. [Website] 23 Apr. 2013 [cited 201304 May]; Available from: <http://www.cdc.gov/plague/maps/>.

5. Butler, T., Plague gives surprises in the first decade of the 21st century in the United States and worldwide. Am J Trop Med Hyg, 2013. 89(4): p. 788-93.

6. Natalie Kwit, D.C.N., MD; Kiersten Kugeler, PhD; Jeannine Petersen, PhD; Lydia Plante, MSPH; Hayley Yaglom, MPH; Vicki Kramer, PhD; Benjamin Schwartz, MD; Jennifer House, DVM; Leah Colton, PhD; Amanda Feldpausch, MPH; Cherie Drenzek, DVM; Joan Baumbach, MD; Mark DiMenna, PhD; Emily Fisher, MD; Emilio Debess, DVM; Danielle Buttke, DVM; Matthew Weinburke, MPH; Christopher Percy, MD; Martin Schriefer, PhD; Ken Gage, PhD; Paul Mead, MD, Human Plague - United States, 2015, in MMWR Morbidity and Mortality Weekly Report. 2015: Centers for Disease Control and Prevention.

7. CDC, Possession, Use, and Transfer of Select Agents and Toxins; Biennial Review; Final Rule. 2012: Federal Register. p. 61083-61115.

8. Hull, H.F., J.M. Montes, and J.M. Mann, Septicemic plague in New Mexico. J Infect Dis, 1987. 155(1): p. 113-8.

9. Doll, J.M., et al., Cat-transmitted fatal pneumonic plague in a person who traveled from Colorado to Arizona. Am J Trop Med Hyg, 1994. 51(1): p. 109-14.

10. von Reyn, C.F., et al., Epidemiologic and clinical features of an outbreak of bubonic plague in New Mexico. J Infect Dis, 1977. 136(4): p. 489-94.

11. Crook, L.D. and B. Tempest, Plague. A clinical review of 27 cases. Arch Intern Med, 1992. 152(6): p. 1253-6.

12. Conrad, F.G., F.R. LeCocq, and R. Krain, A recent epidemic of plague in Vietnam. Arch Intern Med, 1968. 122(3): p. 193-8.

13. Butler, T., The black death past and present. 1. Plague in the 1980s. Trans R Soc Trop Med Hyg, 1989. 83(4): p. 458-60.

14. Inglesby, T.V., et al., Plague as a biological weapon: medical and public health management. Working Group on Civilian Biodefense. Jama, 2000. 283(17): p. 2281-90.

15. Abbott, R.C., and Rocke, T.E. , Plague: U.S. Geological Survey Circular 1372. 2012: p. 79 p.

16. Achtman, M., et al., Microevolution and history of the plague bacillus, Yersinia pestis. Proc Natl Acad Sci U S A, 2004. 101(51): p. 17837-42.

17. Chain, P.S., et al., Insights into the evolution of Yersinia pestis through whole-genome comparison with Yersinia pseudotuberculosis. Proc Natl Acad Sci U S A, 2004. 101(38): p. 13826-31.

18. Parkhill, J., et al., Genome sequence of Yersinia pestis, the causative agent of plague. Nature, 2001. 413(6855): p. 523-7.

19. Williamson, E.D. and P.C. Oyston, The natural history and incidence of Yersinia pestis and prospects for vaccination. J Med Microbiol, 2012. 61(Pt 7): p. 911-8. 
20. Butler, T., Plague history: Yersin's discovery of the causative bacterium in 1894 enabled, in the subsequent century, scientific progress in understanding the disease and the development of treatments and vaccines. Clin Microbiol Infect, 2014. 20(3): p. 202-9.

21. Bos, K.I., et al., A draft genome of Yersinia pestis from victims of the Black Death. Nature, 2011. 478(7370): p. 506-10.

22. Seifert, L., et al., Genotyping Yersinia pestis in Historical Plague: Evidence for Long-Term Persistence of $Y$. pestis in Europe from the 14th to the 17th Century. PLoS One, 2016. 11(1): p. e0145194.

23. Harbeck, M., et al., Yersinia pestis DNA from Skeletal Remains from the 6(th) Century AD Reveals Insights into Justinianic Plague. PLoS Pathog, 2013. 9(5): p. e1003349.

24. Muhlenkamp, M., et al., Yersinia adhesin A (YadA)--beauty \& beast. Int J Med Microbiol, 2015. 305(2): p. 252-8.

25. Bialas, N., et al., Bacterial cell surface structures in Yersinia enterocolitica. Arch Immunol Ther Exp (Warsz), 2012. 60(3): p. 199-209.

26. Mikula, K.M., R. Kolodziejczyk, and A. Goldman, Yersinia infection tools-characterization of structure and function of adhesins. Front Cell Infect Microbiol, 2012. 2: p. 169.

27. Cowan, C., et al., Invasion of epithelial cells by Yersinia pestis: evidence for a Y. pestisspecific invasin. Infect Immun, 2000. 68(8): p. 4523-30.

28. Gillenius, E. and C.F. Urban, The adhesive protein invasin of Yersinia pseudotuberculosis induces neutrophil extracellular traps via beta1 integrins. Microbes Infect, 2015.

29. Grassl, G.A., et al., Interaction of Yersinia enterocolitica with epithelial cells: invasin beyond invasion. Int J Med Microbiol, 2003. 293(1): p. 41-54.

30. Suomalainen, M., et al., Using every trick in the book: the Pla surface protease of Yersinia pestis. Adv Exp Med Biol, 2007. 603: p. 268-78.

31. Zhang, S.S., et al., Plasminogen activator Pla of Yersinia pestis utilizes murine DEC-205 (CD205) as a receptor to promote dissemination. J Biol Chem, 2008. 283(46): p. 3151121.

32. Trosky, J.E., A.D. Liverman, and K. Orth, Yersinia outer proteins: Yops. Cell Microbiol, 2008. 10(3): p. 557-65.

33. Viboud, G.I. and J.B. Bliska, Yersinia outer proteins: role in modulation of host cell signaling responses and pathogenesis. Annu Rev Microbiol, 2005. 59: p. 69-89.

34. Caulfield, A.J. and W.W. Lathem, Substrates of the plasminogen activator protease of Yersinia pestis. Adv Exp Med Biol, 2012. 954: p. 253-60.

35. Hinnebusch, B.J., E.R. Fischer, and T.G. Schwan, Evaluation of the role of the Yersinia pestis plasminogen activator and other plasmid-encoded factors in temperaturedependent blockage of the flea. J Infect Dis, 1998. 178(5): p. 1406-15.

36. Easterbrook, T.J., et al., Studies on the immunogenicity of the Pla protein from Yersinia pestis. Contrib Microbiol Immunol, 1995. 13: p. 214-5.

37. Hu, P., et al., Structural organization of virulence-associated plasmids of Yersinia pestis. J Bacteriol, 1998. 180(19): p. 5192-202.

38. Lindler, L.E., et al., Complete DNA sequence and detailed analysis of the Yersinia pestis KIM5 plasmid encoding murine toxin and capsular antigen. Infect Immun, 1998. 66(12): p. 5731-42.

39. Drozdov, I.G., et al., Virulent non-capsulate Yersinia pestis variants constructed by insertion mutagenesis. J Med Microbiol, 1995. 42(4): p. 264-8.

40. Chouikha, I. and B.J. Hinnebusch, Yersinia-flea interactions and the evolution of the arthropod-borne transmission route of plague. Curr Opin Microbiol, 2012. 
41. Chouikha, I. and B.J. Hinnebusch, Yersinia--flea interactions and the evolution of the arthropod-borne transmission route of plague. Curr Opin Microbiol, 2012. 15(3): p. 23946.

42. Hinnebusch, B.J., The evolution of flea-borne transmission in Yersinia pestis. Curr Issues Mol Biol, 2005. 7(2): p. 197-212.

43. Williamson, E.D. and P.C. Oyston, Protecting against plague: towards a next-generation vaccine. Clin Exp Immunol, 2013. 172(1): p. 1-8.

44. Sun, W. and R. Curtiss, Rational considerations about development of live attenuated Yersinia pestis vaccines. Curr Pharm Biotechnol, 2013. 14(10): p. 878-86.

45. Kartman, L., M.I. Goldenberg, and W.T. Hubbert, Recent observations on the epidemiology of plague in the United States. Am J Public Health Nations Health, 1966. 56(9): p. 1554-69.

46. Hinnebusch, B.J., Bubonic plague: a molecular genetic case history of the emergence of an infectious disease. J Mol Med (Berl), 1997. 75(9): p. 645-52.

47. Hinnebusch, B.J. and D.L. Erickson, Yersinia pestis biofilm in the flea vector and its role in the transmission of plague. Curr Top Microbiol Immunol, 2008. 322: p. 229-48.

48. Hinnebusch, B.J., R.D. Perry, and T.G. Schwan, Role of the Yersinia pestis hemin storage (hms) locus in the transmission of plague by fleas. Science, 1996. 273(5273): p. 367-70.

49. St John, A.L., et al., S1P-Dependent Trafficking of Intracellular Yersinia pestis through Lymph Nodes Establishes Buboes and Systemic Infection. Immunity, 2014. 41(3): p. 44050.

50. Hinnebusch, B.J., et al., Role of the Yersinia pestis Ail protein in preventing a protective polymorphonuclear leukocyte response during bubonic plague. Infect Immun, 2011. 79(12): p. 4984-9.

51. Spinner, J.L., et al., Yersinia pestis survival and replication within human neutrophil phagosomes and uptake of infected neutrophils by macrophages. J Leukoc Biol, 2013.

52. Ye, Z., et al., Gr1+ cells control growth of YopM-negative yersinia pestis during systemic plague. Infect Immun, 2009. 77(9): p. 3791-806.

53. Janssen, W.A. and M.J. Surgalla, Plague bacillus: survival within host phagocytes. Science, 1969. 163(3870): p. 950-2.

54. Straley, S.C. and P.A. Harmon, Growth in mouse peritoneal macrophages of Yersinia pestis lacking established virulence determinants. Infect Immun, 1984. 45(3): p. 649-54.

55. Straley, S.C. and P.A. Harmon, Yersinia pestis grows within phagolysosomes in mouse peritoneal macrophages. Infect Immun, 1984. 45(3): p. 655-9.

56. Oyston, P.C., et al., The response regulator PhoP is important for survival under conditions of macrophage-induced stress and virulence in Yersinia pestis. Infect Immun, 2000. 68(6): p. 3419-25.

57. Pujol, C. and J.B. Bliska, The ability to replicate in macrophages is conserved between Yersinia pestis and Yersinia pseudotuberculosis. Infect Immun, 2003. 71(10): p. 5892-9.

58. Lukaszewski, R.A., et al., Pathogenesis of Yersinia pestis infection in BALB/c mice: effects on host macrophages and neutrophils. Infect Immun, 2005. 73(11): p. 7142-50.

59. Pujol, C., et al., Yersinia pestis can reside in autophagosomes and avoid xenophagy in murine macrophages by preventing vacuole acidification. Infect Immun, 2009. 77(6): p. 2251-61.

60. Bliska, J., Survival and Trafficking of Yersinia pestis in Non-acidified Phagosomes in Murine Macrophages, in The Challenge of Highly Pathogenic Microorganisms, A. Shafferman, A. Ordentlich, and B. Velan, Editors. 2010, Springer Netherlands. p. 31-44. 
61. Connor, M.G., et al., Yersinia pestis Requires Host Rab1b for Survival in Macrophages. PLoS Pathog, 2015. 11(10): p. e1005241.

62. Ponnusamy, D. and K.D. Clinkenbeard, Yersinia pestis intracellular parasitism of macrophages from hosts exhibiting high and low severity of plague. PLoS One, 2012. 7(7): p. 27.

63. Pujol, C. and J.B. Bliska, Turning Yersinia pathogenesis outside in: subversion of macrophage function by intracellular yersiniae. Clin Immunol, 2005. 114(3): p. 216-26.

64. Marketon, M.M., et al., Plague bacteria target immune cells during infection. Science, 2005. 309(5741): p. 1739-41.

65. Balada-Llasat, J.M. and J. Mecsas, Yersinia has a tropism for B and T cell zones of lymph nodes that is independent of the type III secretion system. PLoS Pathog, 2006. 2(9): p. e86.

66. Merritt, P.M., et al., Yersinia pestis targets neutrophils via complement receptor 3. Cell Microbiol, 2014.

67. Knirel, Y.A. and A.P. Anisimov, Lipopolysaccharide of Yersinia pestis, the Cause of Plague: Structure, Genetics, Biological Properties. Acta Naturae, 2012. 4(3): p. 46-58.

68. Telepnev, M.V., et al., Tetraacylated lipopolysaccharide of Yersinia pestis can inhibit multiple Toll-like receptor-mediated signaling pathways in human dendritic cells. J Infect Dis, 2009. 200(11): p. 1694-702.

69. Maeshima, N. and R.C. Fernandez, Recognition of lipid A variants by the TLR4-MD-2 receptor complex. Front Cell Infect Microbiol, 2013. 3: p. 3.

70. Poltorak, A., et al., Defective LPS signaling in $\mathrm{C} 3 \mathrm{H} / \mathrm{HeJ}$ and $\mathrm{C} 57 \mathrm{BL} / 10 \mathrm{SCCr}$ mice: mutations in T/r4 gene. Science, 1998. 282(5396): p. 2085-8.

71. Zanoni, I., et al., CD14 controls the LPS-induced endocytosis of Toll-like receptor 4. Cell, 2011. 147(4): p. 868-80.

72. Andrews, G.P., et al., Fraction 1 capsular antigen (F1) purification from Yersinia pestis CO92 and from an Escherichia coli recombinant strain and efficacy against lethal plague challenge. Infect Immun, 1996. 64(6): p. 2180-7.

73. Soliakov, A., et al., The structure of Yersinia pestis Caf1 polymer in free and adjuvant bound states. Vaccine, 2010. 28(35): p. 5746-54.

74. Tito, M.A., et al., Macromolecular organization of the Yersinia pestis capsular F1 antigen: insights from time-of-flight mass spectrometry. Protein Sci, 2001. 10(11): p. 2408-13.

75. Cavanaugh, D.C. and R. Randall, The role of multiplication of Pasteurella pestis in mononuclear phagocytes in the pathogenesis of flea-borne plague. J Immunol, 1959. 83: p. 348-63.

76. Bacot, A.W. and C.J. Martin, LXVII. Observations on the mechanism of the transmission of plague by fleas. J Hyg (Lond), 1914. 13(Suppl): p. 423-39.

77. Du, Y., E. Galyov, and A. Forsberg, Genetic analysis of virulence determinants unique to Yersinia pestis. Contrib Microbiol Immunol, 1995. 13: p. 321-4.

78. Chromy, B.A., et al., Proteomic characterization of Yersinia pestis virulence. J Bacteriol, 2005. 187(23): p. 8172-80.

79. Karlyshev, A.V., et al., Caf1R gene and its role in the regulation of capsule formation of $Y$. pestis. FEBS Lett, 1992. 305(1): p. 37-40.

80. Vagima, Y., et al., Circumventing Y. pestis Virulence by Early Recruitment of Neutrophils to the Lungs during Pneumonic Plague. PLoS Pathog, 2015. 11(5): p. e1004893. 
81. Spinner, J.L. and B.J. Hinnebusch, The life stage of Yersinia pestis in the flea vector confers increased resistance to phagocytosis and killing by murine polymorphonuclear leukocytes. Adv Exp Med Biol, 2012. 954: p. 159-63.

82. Burrows, T.W. and G.A. Bacon, The basis of virulence in Pasteurella pestis: the development of resistance to phagocytosis in vitro. Br J Exp Pathol, 1956. 37(3): p. 28699.

83. Grabenstein, J.P., et al., The response regulator PhoP of Yersinia pseudotuberculosis is important for replication in macrophages and for virulence. Infect Immun, 2004. 72(9): p. 4973-84.

84. Finegold, M.J., Pneumonic plague in monkeys. An electron microscopic study. Am J Pathol, 1969. 54(2): p. 167-85.

85. Zhang, Y. and J.B. Bliska, Role of macrophage apoptosis in the pathogenesis of Yersinia. Curr Top Microbiol Immunol, 2005. 289: p. 151-73.

86. Grabenstein, J.P., et al., Characterization of phagosome trafficking and identification of PhoP-regulated genes important for survival of Yersinia pestis in macrophages. Infect Immun, 2006. 74(7): p. 3727-41.

87. Klein, K.A., Elucidation of mechanisms of Yersinia pestis survival in macrophages by examination of phagosomal acidification and negative selection screening, in Genetics. 2009, Stony Brook University: The official electronic file of this thesis or dissertation is maintained by the University Libraries on behalf of The Graduate School at Stony Brook University. p. 116.

88. Moreau, K., et al., Autophagosomes can support Yersinia pseudotuberculosis replication in macrophages. Cell Microbiol, 2010. 12(8): p. 1108-23.

89. Ligeon, L.A., et al., Role of VAMP3 and VAMP7 in the commitment of Yersinia pseudotuberculosis to LC3-associated pathways involving single-or double-membrane vacuoles. Autophagy, 2014. 10(9).

90. Bozue, J., et al., The role of the phoPQ operon in the pathogenesis of the fully virulent CO92 strain of Yersinia pestis and the IP32953 strain of Yersinia pseudotuberculosis. Microb Pathog, 2011. 50(6): p. 314-21.

91. Rust, J.H., Jr., et al., The role of domestic animals in the epidemiology of plague. I. Experimental infection of dogs and cats. J Infect Dis, 1971. 124(5): p. 522-6.

92. Desjardins, M., et al., Biogenesis of phagolysosomes proceeds through a sequential series of interactions with the endocytic apparatus. J Cell Biol, 1994. 124(5): p. 677-88.

93. Roberts, E.A., et al., Higher order Rab programming in phagolysosome biogenesis. J Cell Biol, 2006. 174(7): p. 923-9.

94. Kinchen, J.M. and K.S. Ravichandran, Phagosome maturation: going through the acid test. Nat Rev Mol Cell Biol, 2008. 9(10): p. 781-95.

95. Vieira, O.V., R.J. Botelho, and S. Grinstein, Phagosome maturation: aging gracefully. Biochem J, 2002. 366(Pt 3): p. 689-704.

96. Sarantis, H., et al., Yersinia entry into host cells requires Rab5-dependent dephosphorylation of PI(4,5)P(2) and membrane scission. Cell Host Microbe, 2012. 11(2): p. 117-28.

97. Luzio, J.P., N.A. Bright, and P.R. Pryor, The role of calcium and other ions in sorting and delivery in the late endocytic pathway. Biochem Soc Trans, 2007. 35(Pt 5): p. 1088-91.

98. Luzio, J.P., S.R. Gray, and N.A. Bright, Endosome-lysosome fusion. Biochem Soc Trans, 2010. 38(6): p. 1413-6.

99. Luzio, J.P., et al., ESCRT proteins and the regulation of endocytic delivery to lysosomes. Biochem Soc Trans, 2009. 37(Pt 1): p. 178-80. 
100. Luzio, J.P., P.R. Pryor, and N.A. Bright, Lysosomes: fusion and function. Nat Rev Mol Cell Biol, 2007. 8(8): p. 622-32.

101. Luzio, J.P., et al., Membrane traffic to and from lysosomes. Biochem Soc Symp, 2005(72): p. 77-86.

102. Stein, M.P., M.P. Muller, and A. Wandinger-Ness, Bacterial pathogens commandeer Rab GTPases to establish intracellular niches. Traffic, 2012. 13(12): p. 1565-88.

103. Brumell, J.H. and M.A. Scidmore, Manipulation of rab GTPase function by intracellular bacterial pathogens. Microbiol Mol Biol Rev, 2007. 71(4): p. 636-52.

104. Hilbi, H., et al., Beyond Rab GTPases: activates the small GTPase Ran to promote microtubule polymerization, pathogen vacuole motility, and infection. Small GTPases, 2014. 5(1).

105. Bloom, B.R. and C.J. Murray, Tuberculosis: commentary on a reemergent killer. Science, 1992. 257(5073): p. 1055-64.

106. Deretic, V., Autophagy, an immunologic magic bullet: Mycobacterium tuberculosis phagosome maturation block and how to bypass it. Future Microbiol, 2008. 3(5): p. 51724.

107. Seto, S., et al., Dissection of Rab7 localization on Mycobacterium tuberculosis phagosome. Biochem Biophys Res Commun, 2009. 387(2): p. 272-7.

108. Seto, S., K. Tsujimura, and Y. Koide, Rab GTPases regulating phagosome maturation are differentially recruited to mycobacterial phagosomes. Traffic, 2011. 12(4): p. 407-20.

109. Fratti, R.A., et al., Role of phosphatidylinositol 3-kinase and Rab5 effectors in phagosomal biogenesis and mycobacterial phagosome maturation arrest. J Cell Biol, 2001. 154(3): p. 631-44.

110. Fratti, R.A., et al., Mycobacterium tuberculosis glycosylated phosphatidylinositol causes phagosome maturation arrest. Proc Natl Acad Sci U S A, 2003. 100(9): p. 5437-42.

111. Santos, J.C. and J. Enninga, At the crossroads: communication of bacteria-containing vacuoles with host organelles. Cell Microbiol, 2016.

112. Price, C.T., A.M. Richards, and Y. Abu Kwaik, Nutrient generation and retrieval from the host cell cytosol by intra-vacuolar Legionella pneumophila. Front Cell Infect Microbiol, 2014. 4: p. 111.

113. Chen, Y., et al., Structural basis for Rab1 de-AMPylation by the Legionella pneumophila effector SidD. PLoS Pathog, 2013. 9(5): p. e1003382.

114. Del Campo, C.M., et al., Structural basis for PI(4)P-specific membrane recruitment of the Legionella pneumophila effector DrrA/SidM. Structure, 2014. 22(3): p. 397-408.

115. Hardiman, C.A. and C.R. Roy, AMPylation is critical for Rab1 localization to vacuoles containing Legionella pneumophila. MBio, 2014. 5(1): p. e01035-13.

116. Horenkamp, F.A., et al., Legionella pneumophila subversion of host vesicular transport by SidC effector proteins. Traffic, 2014. 15(5): p. 488-99.

117. Ingmundson, A., et al., Legionella pneumophila proteins that regulate Rab1 membrane cycling. Nature, 2007. 450(7168): p. 365-9.

118. Kagan, J.C., et al., Legionella subvert the functions of Rab1 and Sec22b to create $a$ replicative organelle. J Exp Med, 2004. 199(9): p. 1201-11.

119. Machner, M.P. and R.R. Isberg, Targeting of host Rab GTPase function by the intravacuolar pathogen Legionella pneumophila. Dev Cell, 2006. 11(1): p. 47-56.

120. Mishra, A.K., et al., The Legionella pneumophila GTPase activating protein LepB accelerates Rab1 deactivation by a non-canonical hydrolytic mechanism. J Biol Chem, 2013. 288(33): p. 24000-11. 
121. Muller, M.P., et al., The Legionella effector protein DrrA AMPylates the membrane traffic regulator Rab1b. Science, 2010. 329(5994): p. 946-9.

122. Murata, T., et al., The Legionella pneumophila effector protein DrrA is a Rab1 guanine nucleotide-exchange factor. Nat Cell Biol, 2006. 8(9): p. 971-7.

123. Neunuebel, M.R., et al., Legionella pneumophila LidA affects nucleotide binding and activity of the host GTPase Rab1. J Bacteriol, 2012. 194(6): p. 1389-400.

124. Schoebel, S., et al., Protein LidA from Legionella is a Rab GTPase supereffector. Proc Natl Acad Sci U S A, 2011. 108(44): p. 17945-50.

125. Tan, Y. and Z.Q. Luo, Legionella pneumophila SidD is a deAMPylase that modifies Rab1. Nature, 2011. 475(7357): p. 506-9.

126. Zhu, Y., et al., Structural mechanism of host Rab1 activation by the bifunctional Legionella type IV effector SidM/DrrA. Proc Natl Acad Sci U S A, 2010. 107(10): p. 4699704.

127. Campanacci, V., et al., Structure of the Legionella effector AnkX reveals the mechanism of phosphocholine transfer by the FIC domain. Embo j, 2013. 32(10): p. 1469-77.

128. Mihai Gazdag, E., et al., Mechanism of Rab1b deactivation by the Legionella pneumophila GAP LepB. EMBO Rep, 2013. 14(2): p. 199-205.

129. Tan, Y., R.J. Arnold, and Z.Q. Luo, Legionella pneumophila regulates the small GTPase Rab1 activity by reversible phosphorylcholination. Proc Natl Acad Sci U S A, 2011. 108(52): p. 21212-7.

130. Kuhbacher, A., et al., Genome-Wide siRNA Screen Identifies Complementary Signaling Pathways Involved in Listeria Infection and Reveals Different Actin Nucleation Mechanisms during Listeria Cell Invasion and Actin Comet Tail Formation. MBio, 2015. 6(3): p. e00598-15.

131. McDonough, J.A., et al., Host Pathways Important for Coxiella burnetii Infection Revealed by Genome-Wide RNA Interference Screening. MBio, 2013. 4(1): p. 00606-12.

132. Ooi, Y.S., et al., Genome-Wide RNAi Screen Identifies Novel Host Proteins Required for Alphavirus Entry. PLoS Pathog, 2013. 9(12): p. e1003835.

133. Thornbrough, J.M., et al., Human genome-wide RNAi screen for host factors that modulate intracellular Salmonella growth. PLoS One, 2012. 7(6): p. 11.

134. Zhou, H., et al., Genome-wide RNAi screen in IFN-gamma-treated human macrophages identifies genes mediating resistance to the intracellular pathogen Francisella tularensis. PLoS One, 2012. 7(2): p. e31752.

135. Akimana, C., S. Al-Khodor, and Y. Abu Kwaik, Host factors required for modulation of phagosome biogenesis and proliferation of Francisella tularensis within the cytosol. PLoS One, 2010. 5(6): p. e11025.

136. Kumar, D., et al., Genome-wide analysis of the host intracellular network that regulates survival of Mycobacterium tuberculosis. Cell, 2010. 140(5): p. 731-43.

137. Jayaswal, S., et al., Identification of host-dependent survival factors for intracellular Mycobacterium tuberculosis through an siRNA screen. PLoS Pathog, 2010. 6(4): p. e1000839.

138. Qin, Q.M., et al., RNAi screen of endoplasmic reticulum-associated host factors reveals a role for IRE1alpha in supporting Brucella replication. PLoS Pathog, 2008. 4(7): p. e1000110.

139. Krishnan, M.N., et al., RNA interference screen for human genes associated with West Nile virus infection. Nature, 2008. 455(7210): p. 242-5.

140. Derre, I., et al., RNAi screen in Drosophila cells reveals the involvement of the Tom complex in Chlamydia infection. PLoS Pathog, 2007. 3(10): p. 1446-58. 
141. Agaisse, H., et al., Genome-wide RNAi screen for host factors required for intracellular bacterial infection. Science, 2005. 309(5738): p. 1248-51.

142. Philips, J.A., E.J. Rubin, and N. Perrimon, Drosophila RNAi screen reveals CD 36 family member required for mycobacterial infection. Science, 2005. 309(5738): p. 1251-3.

143. Sharma, S. and A. Rao, RNAi screening: tips and techniques. Nat Immunol, 2009. 10(8): p. 799-804.

144. Echeverri, C.J. and N. Perrimon, High-throughput RNAi screening in cultured cells: a user's guide. Nat Rev Genet, 2006. 7(5): p. 373-84.

145. Sledz, C.A. and B.R. Williams, RNA interference and double-stranded-RNA-activated pathways. Biochem Soc Trans, 2004. 32(Pt 6): p. 952-6.

146. Elbashir, S.M., W. Lendeckel, and T. Tuschl, RNA interference is mediated by 21- and 22nucleotide RNAs. Genes Dev, 2001. 15(2): p. 188-200.

147. Brummelkamp, T.R., R. Bernards, and R. Agami, A system for stable expression of short interfering RNAs in mammalian cells. Science, 2002. 296(5567): p. 550-3.

148. Boutros, M., F. Heigwer, and C. Laufer, Microscopy-Based High-Content Screening. Cell, 2015. 163(6): p. 1314-25.

149. Birmingham, A., et al., Statistical methods for analysis of high-throughput RNA interference screens. Nat Methods, 2009. 6(8): p. 569-75.

150. Malo, N., et al., Statistical practice in high-throughput screening data analysis. Nat Biotechnol, 2006. 24(2): p. 167-75.

151. Sun, Y., et al., Development of bioluminescent bioreporters for in vitro and in vivo tracking of Yersinia pestis. PLoS One, 2012. 7(10): p. e47123.

152. Pujol, C., et al., Replication of Yersinia pestis in interferon gamma-activated macrophages requires ripA, a gene encoded in the pigmentation locus. Proc Natl Acad Sci U S A, 2005. 102(36): p. 12909-14.

153. Andrews, G.P. and A.T. Maurelli, mxiA of Shigella flexneri $2 a$, which facilitates export of invasion plasmid antigens, encodes a homolog of the low-calcium-response protein, LcrD, of Yersinia pestis. Infect Immun, 1992. 60(8): p. 3287-95.

154. Close, D., et al., The evolution of the bacterial luciferase gene cassette (lux) as a realtime bioreporter. Sensors (Basel), 2012. 12(1): p. 732-52.

155. Meighen, E.A., Molecular biology of bacterial bioluminescence. Microbiol Rev, 1991. 55(1): p. 123-42.

156. Butler, T., Plague and Other Yersinia Infections. Current Topics in Infectious Disease, ed. W.B.G.I.a.T.C. Merigan. 1983, New York: Plenum Medical Book Company. 1-220.

157. Kauppi, A.M., et al., Targeting bacterial virulence: inhibitors of type III secretion in Yersinia. Chem Biol, 2003. 10(3): p. 241-9.

158. Pan, N.J., et al., Targeting type III secretion in Yersinia pestis. Antimicrob Agents Chemother, 2009. 53(2): p. 385-92.

159. Trcek, J., T.M. Fuchs, and K. Trulzsch, Analysis of Yersinia enterocolitica invasin expression in vitro and in vivo using a novel luxCDABE reporter system. Microbiology, 2010. 156(Pt 9): p. 2734-45.

160. Uliczka, F., et al., Monitoring of gene expression in bacteria during infections using an adaptable set of bioluminescent, fluorescent and colorigenic fusion vectors. PLoS One, 2011. 6(6): p. e20425.

161. Strong, P.C., et al., Identification and characterisation of a novel adhesin Ifp in Yersinia pseudotuberculosis. BMC Microbiol, 2011. 11: p. 85.

162. Trcek, J., K. Berschl, and K. Trulzsch, In vivo analysis of Yersinia enterocolitica infection using luxCDABE. FEMS Microbiol Lett, 2010. 307(2): p. 201-6. 
163. Thorslund, S.E., et al., The RACK1 signaling scaffold protein selectively interacts with Yersinia pseudotuberculosis virulence function. PLoS One, 2011. 6(2): p. e16784.

164. Nham, T., et al., Imaging of bubonic plague dynamics by in vivo tracking of bioluminescent Yersinia pestis. PLoS One, 2012. 7(4): p. e34714.

165. Warawa, J.M., et al., Bioluminescent diagnostic imaging to characterize altered respiratory tract colonization by the burkholderia pseudomallei capsule mutant. Front Microbiol, 2011. 2: p. 133.

166. Massey, S., et al., In vivo Bioluminescence Imaging of Burkholderia mallei Respiratory Infection and Treatment in the Mouse Model. Front Microbiol, 2011. 2: p. 174.

167. Bina, X.R., M.A. Miller, and J.E. Bina, Construction of a bioluminescence reporter plasmid for Francisella tularensis. Plasmid, 2010. 64(3): p. 156-61.

168. Miller, M.A., et al., Visualization of murine intranasal dosing efficiency using luminescent Francisella tularensis: effect of instillation volume and form of anesthesia. PLoS One, 2012. 7(2): p. e31359.

169. Lawrenz, M.B., Model systems to study plague pathogenesis and develop new therapeutics. Front Microbiol, 2010. 1: p. 119.

170. Lane, M.C., et al., Expression of flagella is coincident with uropathogenic Escherichia coli ascension to the upper urinary tract. Proc Natl Acad Sci U S A, 2007. 104(42): p. 1666974.

171. Choi, K.H., et al., A Tn7-based broad-range bacterial cloning and expression system. Nat Methods, 2005. 2(6): p. 443-8.

172. Bland, D.M., et al., Novel genetic tools for diaminopimelic acid selection in virulence studies of Yersinia pestis. PLoS One, 2011. 6(3): p. e17352.

173. Harrell, M.I., B.M. Iritani, and A. Ruddell, Lymph node mapping in the mouse. J Immunol Methods, 2008. 332(1-2): p. 170-4.

174. Van den Broeck, W., A. Derore, and P. Simoens, Anatomy and nomenclature of murine lymph nodes: Descriptive study and nomenclatory standardization in BALB/CAnNCrl mice. J Immunol Methods, 2006. 312(1-2): p. 12-9.

175. Lathem, W.W., et al., Progression of primary pneumonic plague: a mouse model of infection, pathology, and bacterial transcriptional activity. Proc. Natl. Acad. Sci. USA, 2005. 102(49): p. 17786-91.

176. Lathem, W.W., et al., A plasminogen-activating protease specifically controls the development of primary pneumonic plague. Science, 2007. 315(5811): p. 509-13.

177. Sodeinde, O.A., et al., A surface protease and the invasive character of plague. Science, 1992. 258(5084): p. 1004-7.

178. Welkos, S.L., A.M. Friedlander, and K.J. Davis, Studies on the role of plasminogen activator in systemic infection by virulent Yersinia pestis strain C092. Microb Pathog, 1997. 23(4): p. 211-23.

179. Sebbane, F., et al., Role of the Yersinia pestis plasminogen activator in the incidence of distinct septicemic and bubonic forms of flea-borne plague. Proc Natl Acad Sci U S A, 2006. 103(14): p. 5526-30.

180. Galen, J.E., et al., Optimization of plasmid maintenance in the attenuated live vector vaccine strain Salmonella typhi CVD 908-htrA. Infect. Immun., 1999. 67(12): p. 6424-33.

181. Cathelyn, J.S., et al., RovA, a global regulator of Yersinia pestis, specifically required for bubonic plague. Proc. Natl. Acad. Sci. USA, 2006. 103(36): p. 13514-9.

182. Pujol, C. and J.B. Bliska, The ability to replicate in macrophages is conserved between Yersinia pestis and Yersinia pseudotuberculosis. Infect. Immun., 2003. 71(10): p. 5892-9. 
183. Lawrenz, M.B., J.D. Lenz, and V.L. Miller, A novel autotransporter adhesin is required for efficient colonization during bubonic plague. Infect. Immun., 2009. 77(1): p. 317-26.

184. Gonzalez, R.J., et al., Dissemination of a highly virulent pathogen: tracking the early events that define infection. PLoS Pathog, 2015. 11(1): p. e1004587.

185. Bhuin, T. and J.K. Roy, Rab proteins: the key regulators of intracellular vesicle transport. Exp Cell Res, 2014. 328(1): p. 1-19.

186. Sturgill-Koszycki, S., U.E. Schaible, and D.G. Russell, Mycobacterium-containing phagosomes are accessible to early endosomes and reflect a transitional state in normal phagosome biogenesis. EMBO J, 1996. 15(24): p. 6960-8.

187. Via, L.E., et al., Arrest of mycobacterial phagosome maturation is caused by a block in vesicle fusion between stages controlled by rab5 and rab7. J Biol Chem, 1997. 272(20): p. 13326-31.

188. Campoy, E.M., F.C. Zoppino, and M.I. Colombo, The early secretory pathway contributes to the growth of the Coxiella-replicative niche. Infect Immun, 2011. 79(1): p. 402-13.

189. Derre, I. and R.R. Isberg, Legionella pneumophila replication vacuole formation involves rapid recruitment of proteins of the early secretory system. Infect Immun, 2004. 72(5): p. 3048-53.

190. Dong, N., et al., Structurally distinct bacterial TBC-like GAPs link Arf GTPase to Rab1 inactivation to counteract host defenses. Cell, 2012. 150(5): p. 1029-41.

191. Huang, B., et al., The Anaplasma phagocytophilum-occupied vacuole selectively recruits Rab-GTPases that are predominantly associated with recycling endosomes. Cell Microbiol, 2010. 12(9): p. 1292-307.

192. Huang, J., et al., Antibacterial autophagy occurs at PI(3)P-enriched domains of the endoplasmic reticulum and requires Rab1 GTPase. Autophagy, 2011. 7(1): p. 17-26.

193. Neunuebel, M.R., et al., De-AMPylation of the small GTPase Rab1 by the pathogen Legionella pneumophila. Science, 2011. 333(6041): p. 453-6.

194. Rzomp, K.A., et al., Rab GTPases are recruited to chlamydial inclusions in both a speciesdependent and species-independent manner. Infect Immun, 2003. 71(10): p. 5855-70.

195. Hardiman, C.A., et al., The role of Rab GTPases in the transport of vacuoles containing Legionella pneumophila and Coxiella burnetii. Biochem Soc Trans, 2012. 40(6): p. 13539.

196. Tisdale, E.J., et al., GTP-binding mutants of rab1 and rab2 are potent inhibitors of vesicular transport from the endoplasmic reticulum to the Golgi complex. J Cell Biol, 1992. 119(4): p. 749-61.

197. Touchot, N., et al., Biochemical properties of the YPT-related rab1B protein. Comparison with rab1A. FEBS Lett, 1989. 256(1-2): p. 79-84.

198. Plutner, H., et al., Rab1b regulates vesicular transport between the endoplasmic reticulum and successive Golgi compartments. J Cell Biol, 1991. 115(1): p. 31-43.

199. Ao, X., L. Zou, and Y. Wu, Regulation of autophagy by the Rab GTPase network. Cell Death Differ, 2014. 21(3): p. 348-58.

200. Huang, J. and J.H. Brumell, Bacteria-autophagy interplay: a battle for survival. Nat Rev Microbiol, 2014.

201. Mukhopadhyay, A., J.A. Quiroz, and A.W. Wolkoff, Rab1a regulates sorting of early endocytic vesicles. Am J Physiol Gastrointest Liver Physiol, 2014. 306(5): p. G412-24.

202. Mukhopadhyay, A., et al., Proteomic analysis of endocytic vesicles: Rab1a regulates motility of early endocytic vesicles. J Cell Sci, 2011. 124(Pt 5): p. 765-75. 
203. Arasaki, K., D.K. Toomre, and C.R. Roy, The Legionella pneumophila effector DrrA is sufficient to stimulate SNARE-dependent membrane fusion. Cell Host Microbe, 2012. 11(1): p. 46-57.

204. Arasaki, K. and C.R. Roy, Legionella pneumophila promotes functional interactions between plasma membrane syntaxins and Sec22b. Traffic, 2010. 11(5): p. 587-600.

205. Misselwitz, B., et al., RNAi screen of Salmonella invasion shows role of COPI in membrane targeting of cholesterol and Cdc42. Mol Syst Biol, 2011. 7: p. 474.

206. Galen, J.E., et al., Optimization of plasmid maintenance in the attenuated live vector vaccine strain Salmonella typhi CVD 908-htrA. Infect Immun, 1999. 67(12): p. 6424-33.

207. Kagan, J.C. and C.R. Roy, Legionella phagosomes intercept vesicular traffic from endoplasmic reticulum exit sites. Nat Cell Biol, 2002. 4(12): p. 945-54.

208. Itoh, T., et al., Screening for target Rabs of TBC (Tre-2/Bub2/Cdc16) domain-containing proteins based on their Rab-binding activity. Genes Cells, 2006. 11(9): p. 1023-37.

209. Satoh, A., et al., Golgin-84 is a rab1 binding partner involved in Golgi structure. Traffic, 2003. 4(3): p. 153-61.

210. Martinez, O. and B. Goud, Rab proteins. Biochim Biophys Acta, 1998. 1404(1-2): p. 10112.

211. Horwitz, M.A. and F.R. Maxfield, Legionella pneumophila inhibits acidification of its phagosome in human monocytes. J Cell Biol, 1984. 99(6): p. 1936-43.

212. Sturgill-Koszycki, S. and M.S. Swanson, Legionella pneumophila replication vacuoles mature into acidic, endocytic organelles. J Exp Med, 2000. 192(9): p. 1261-72.

213. Ortiz Sandoval, C. and T. Simmen, Rab proteins of the endoplasmic reticulum: functions and interactors. Biochem Soc Trans, 2012. 40(6): p. 1426-32.

214. Liu, S. and B. Storrie, Are Rab proteins the link between Golgi organization and membrane trafficking? Cell Mol Life Sci, 2012. 69(24): p. 4093-106.

215. Gutierrez, M.G., et al., Autophagy induction favours the generation and maturation of the Coxiella-replicative vacuoles. Cell Microbiol, 2005. 7(7): p. 981-93.

216. Niu, H., M. Yamaguchi, and Y. Rikihisa, Subversion of cellular autophagy by Anaplasma phagocytophilum. Cell Microbiol, 2008. 10(3): p. 593-605.

217. Newton, H.J., J.A. McDonough, and C.R. Roy, Effector protein translocation by the Coxiella burnetii Dot/Icm type IV secretion system requires endocytic maturation of the pathogen-occupied vacuole. PLoS One, 2013. 8(1): p. e54566.

218. Wieland, H., F. Goetz, and B. Neumeister, Phagosomal acidification is not a prerequisite for intracellular multiplication of Legionella pneumophila in human monocytes. J Infect Dis, 2004. 189(9): p. 1610-4.

219. Benes, P., V. Vetvicka, and M. Fusek, Cathepsin D--many functions of one aspartic protease. Crit Rev Oncol Hematol, 2008. 68(1): p. 12-28.

220. Saftig, P. and J. Klumperman, Lysosome biogenesis and lysosomal membrane proteins: trafficking meets function. Nat Rev Mol Cell Biol, 2009. 10(9): p. 623-35.

221. Xu, H. and D. Ren, Lysosomal Physiology. Annu Rev Physiol, 2015. 77: p. 57-80.

222. Machner, M.P. and R.R. Isberg, A bifunctional bacterial protein links GDI displacement to Rab1 activation. Science, 2007. 318(5852): p. 974-7.

223. Mukherjee, S., et al., Modulation of Rab GTPase function by a protein phosphocholine transferase. Nature, 2011. 477(7362): p. 103-6.

224. Abu Kwaik, Y., B.I. Eisenstein, and N.C. Engleberg, Phenotypic modulation by Legionella pneumophila upon infection of macrophages. Infect Immun, 1993. 61(4): p. 1320-9. 
225. Al-Khodor, S., et al., A Dot/lcm-translocated ankyrin protein of Legionella pneumophila is required for intracellular proliferation within human macrophages and protozoa. Mol Microbiol, 2008. 70(4): p. 908-23.

226. Pedersen, L.L., et al., HtrA homologue of Legionella pneumophila: an indispensable element for intracellular infection of mammalian but not protozoan cells. Infect Immun, 2001. 69(4): p. 2569-79.

227. Gibson, D.G., et al., Enzymatic assembly of DNA molecules up to several hundred kilobases. Nat Methods, 2009. 6(5): p. 343-5.

228. Livak, K.J. and T.D. Schmittgen, Analysis of relative gene expression data using real-time quantitative PCR and the 2(-Delta Delta C(T)) Method. Methods, 2001. 25(4): p. 402-8.

229. Kinder, S.A., et al., Cloning of the Yenl restriction endonuclease and methyltransferase from Yersinia enterocolitica serotype 08 and construction of a transformable $R-M+$ mutant. Gene, 1993. 136(1-2): p. 271-5.

230. Kugeler, K.J., et al., Epidemiology of human plague in the United States, 1900-2012. Emerg Infect Dis, 2015. 21(1): p. 16-22.

231. Paskewitz, S.M., Transmission factors for insect-vectored microorganisms. Trends Microbiol, 1997. 5(5): p. 171-3.

232. Hinnebusch, B.J., Biofilm-dependent and biofilm-independent mechanisms of transmission of Yersinia pestis by fleas. Adv Exp Med Biol, 2012. 954: p. 237-43.

233. Shannon, J.G., C.F. Bosio, and B.J. Hinnebusch, Dermal Neutrophil, Macrophage and Dendritic Cell Responses to Yersinia pestis Transmitted by Fleas. PLoS Pathog, 2015. 11(3): p. e1004734.

234. Finegold, M.J., et al., Studies on the pathogenesis of plague. Blood coagulation and tissue responses of Macaca mulatta following exposure to aerosols of Pasteurella pestis. Am J Pathol, 1968. 53(1): p. 99-114.

235. Quan, S.F., T.H. Chen, and K.F. Meyer, Protective action of antibiotics against the toxin of Pasteurella pestis in mice. Proc Soc Exp Biol Med, 1950. 75(2): p. 548-9.

236. Bindea, G., J. Galon, and B. Mlecnik, CluePedia Cytoscape plugin: pathway insights using integrated experimental and in silico data. Bioinformatics, 2013. 29(5): p. 661-3.

237. Bindea, G., et al., ClueGO: a Cytoscape plug-in to decipher functionally grouped gene ontology and pathway annotation networks. Bioinformatics, 2009. 25(8): p. 1091-3.

238. Grant, B.D. and J.G. Donaldson, Pathways and mechanisms of endocytic recycling. Nat Rev Mol Cell Biol, 2009. 10(9): p. 597-608.

239. Li, F. and R.D. Vierstra, Autophagy: a multifaceted intracellular system for bulk and selective recycling. Trends Plant Sci, 2012. 17(9): p. 526-37.

240. Daro, E., et al., Rab4 and cellubrevin define different early endosome populations on the pathway of transferrin receptor recycling. Proc Natl Acad Sci U S A, 1996. 93(18): p. 9559-64.

241. Van Der Sluijs, P., et al., The small GTP-binding protein rab4 is associated with early endosomes. Proc Natl Acad Sci U S A, 1991. 88(14): p. 6313-7.

242. van der Sluijs, P., et al., Expression and properties of Rab4 and its effector rabaptin-4 in endocytic recycling. Methods Enzymol, 2001. 329: p. 111-9.

243. Lapierre, L.A., et al., Myosin vb is associated with plasma membrane recycling systems. Mol Biol Cell, 2001. 12(6): p. 1843-57.

244. Maxfield, F.R. and T.E. McGraw, Endocytic recycling. Nat Rev Mol Cell Biol, 2004. 5(2): p. 121-32.

245. Moulder, J.W., Interaction of chlamydiae and host cells in vitro. Microbiol Rev, 1991. 55(1): p. 143-90. 
246. Rejman Lipinski, A., et al., Rab6 and Rab11 regulate Chlamydia trachomatis

development and golgin-84-dependent Golgi fragmentation. PLoS Pathog, 2009. 5(10):

p. e1000615.

247. Heuer, D., et al., Chlamydia causes fragmentation of the Golgi compartment to ensure reproduction. Nature, 2009. 457(7230): p. 731-5.

248. Guichard, A., et al., Anthrax toxins cooperatively inhibit endocytic recycling by the Rab11/Sec15 exocyst. Nature, 2010. 467(7317): p. 854-8.

249. Beemiller, P., A.D. Hoppe, and J.A. Swanson, A phosphatidylinositol-3-kinase-dependent signal transition regulates ARF1 and ARF6 during Fcgamma receptor-mediated phagocytosis. PLoS Biol, 2006. 4(6): p. e162.

250. Chesneau, L., et al., An ARF6/Rab35 GTPase cascade for endocytic recycling and successful cytokinesis. Curr Biol, 2012. 22(2): p. 147-53.

251. Hurtado-Lorenzo, A., et al., V-ATPase interacts with ARNO and Arf6 in early endosomes and regulates the protein degradative pathway. Nat Cell Biol, 2006. 8(2): p. 124-36.

252. Macia, E., et al., Arf6 negatively controls the rapid recycling of the beta2 adrenergic receptor. J Cell Sci, 2012. 125(Pt 17): p. 4026-35.

253. Repella, T.L., et al., Arf6-Dependent Intracellular Trafficking of Pasteurella multocida Toxin and pH-Dependent Translocation from Late Endosomes. Toxins, 2011. 3(3): p. 21841.

254. Szatmari, Z., et al., Rab11 facilitates cross-talk between autophagy and endosomal pathway through regulation of Hook localization. Mol Biol Cell, 2014. 25(4): p. 522-31.

255. Stark, C., et al., BioGRID: a general repository for interaction datasets. Nucleic Acids Res, 2006. 34(Database issue): p. D535-9.

256. Szklarczyk, D., et al., STRING v10: protein-protein interaction networks, integrated over the tree of life. Nucleic Acids Res, 2015. 43(Database issue): p. D447-52.

257. Shannon, P., et al., Cytoscape: a software environment for integrated models of biomolecular interaction networks. Genome Res, 2003. 13(11): p. 2498-504.

258. Mi, H., et al., PANTHER version 10: expanded protein families and functions, and analysis tools. Nucleic Acids Res, 2016. 44(D1): p. D336-42.

259. Schindelin, J., et al., Fiji: an open-source platform for biological-image analysis. Nat Methods, 2012. 9(7): p. 676-82.

260. Cossart, P. and C.R. Roy, Manipulation of host membrane machinery by bacterial pathogens. Curr Opin Cell Biol, 2010. 22(4): p. 547-54.

261. Ribet, D. and P. Cossart, How bacterial pathogens colonize their hosts and invade deeper tissues. Microbes Infect, 2015. 17(3): p. 173-83.

262. Deuretzbacher, A., et al., Beta1 integrin-dependent engulfment of Yersinia enterocolitica by macrophages is coupled to the activation of autophagy and suppressed by type III protein secretion. J Immunol, 2009. 183(9): p. 5847-60.

263. Isberg, R.R., D.L. Voorhis, and S. Falkow, Identification of invasin: a protein that allows enteric bacteria to penetrate cultured mammalian cells. Cell, 1987. 50(5): p. 769-78.

264. Kreibich, S., et al., Autophagy Proteins Promote Repair of Endosomal Membranes Damaged by the Salmonella Type Three Secretion System 1. Cell Host Microbe, 2015. 18(5): p. 527-37.

265. Kolodziejek, A.M., C.J. Hovde, and S.A. Minnich, Yersinia pestis Ail: multiple roles of a single protein. Front Cell Infect Microbiol, 2012. 2: p. 103.

266. Falkow, S., Bacterial entry into eukaryotic cells. Cell, 1991. 65(7): p. 1099-102.

267. Leo, J.C. and M. Skurnik, Adhesins of human pathogens from the genus Yersinia. Adv Exp Med Biol, 2011. 715: p. 1-15. 
268. Elvin, S.J., et al., Evolutionary genetics: Ambiguous role of CCR5 in Y. pestis infection. Nature, 2004. 430(6998): p. 417.

269. Ng, L.C., et al., The response of murine macrophages to infection with Yersinia pestis as revealed by DNA microarray analysis. Adv Exp Med Biol, 2003. 529: p. 155-60.

270. Zhang, P., et al., Human dendritic cell-specific intercellular adhesion molecule-grabbing nonintegrin (CD209) is a receptor for Yersinia pestis that promotes phagocytosis by dendritic cells. Infect Immun, 2008. 76(5): p. 2070-9.

271. Mecsas, J., et al., Evolutionary genetics: CCR5 mutation and plague protection. Nature, 2004. 427(6975): p. 606.

272. Yang, K., et al., Host Langerin (CD207) is a receptor for Yersinia pestis phagocytosis and promotes dissemination. Immunol Cell Biol, 2015. 93(9): p. 815-24.

273. Yang, K., et al., Host Langerin (CD207) is a receptor for Yersinia pestis phagocytosis and promotes dissemination. Immunol Cell Biol, 2015.

274. Razi, M., E.Y. Chan, and S.A. Tooze, Early endosomes and endosomal coatomer are required for autophagy. J Cell Biol, 2009. 185(2): p. 305-21.

275. Styers, M.L., et al., Depletion of beta-COP reveals a role for COP-I in compartmentalization of secretory compartments and in biosynthetic transport of caveolin-1. Am J Physiol Cell Physiol, 2008. 294(6): p. C1485-98.

276. Schliwa, M., Action of cytochalasin D on cytoskeletal networks. J Cell Biol, 1982. 92(1): p. 79-91.

277. Azouz, N.P., et al., Decoding the regulation of mast cell exocytosis by networks of Rab GTPases. J Immunol, 2012. 189(5): p. 2169-80.

278. Baltierra-Uribe, S.L., et al., Mycobacteria entry and trafficking into endothelial cells. Can J Microbiol, 2014. 60(9): p. 569-77.

279. Croxatto, A. and G. Greub, Early intracellular trafficking of Waddlia chondrophila in human macrophages. Microbiology, 2010. 156(Pt 2): p. 340-55.

280. Finlay, B.B. and S. Falkow, Comparison of the invasion strategies used by Salmonella cholerae-suis, Shigella flexneri and Yersinia enterocolitica to enter cultured animal cells: endosome acidification is not required for bacterial invasion or intracellular replication. Biochimie, 1988. 70(8): p. 1089-99.

281. Garcia-del Portillo, F., et al., Salmonella typhimurium induces selective aggregation and internalization of host cell surface proteins during invasion of epithelial cells. J Cell Sci, 1994. 107 ( Pt 7): p. 2005-20.

282. Guerra, C.R., et al., Cryptococcus neoformans is internalized by receptor-mediated or 'triggered' phagocytosis, dependent on actin recruitment. PLoS One, 2014. 9(2): p. e89250.

283. Hsu, C.R., et al., Klebsiella pneumoniae translocates across the intestinal epithelium via Rho GTPase- and phosphatidylinositol 3-kinase/Akt-dependent cell invasion. Infect Immun, 2015. 83(2): p. 769-79.

284. Kuhn, M., The microtubule depolymerizing drugs nocodazole and colchicine inhibit the uptake of Listeria monocytogenes by P388D1 macrophages. FEMS Microbiol Lett, 1998. 160(1): p. 87-90.

285. Levenhagen, M.A., et al., The role of cytoskeleton, components of inositol phospholipid signaling pathway and iron in Ehrlichia canis in vitro proliferation. Vet Res Commun, 2012. 36(3): p. 195-9.

286. Macdonald, L.J., et al., Coxiella burnetii exploits host cAMP-dependent protein kinase signalling to promote macrophage survival. Cell Microbiol, 2013. 13(10): p. 12213. 
287. Mi, H., et al., Large-scale gene function analysis with the PANTHER classification system. Nat Protoc, 2013. 8(8): p. 1551-66.

288. Watson, P.J., et al., Gamma-COP appendage domain - structure and function. Traffic, 2004. 5(2): p. 79-88.

289. Stenmark, H., Rab GTPases as coordinators of vesicle traffic. Nat Rev Mol Cell Biol, 2009. 10(8): p. 513-25.

290. Stenmark, H. and V.M. Olkkonen, The Rab GTPase family. Genome Biol, 2001. 2(5): p. Reviews3007.

291. Chauvaux, S., et al., Transcriptome analysis of Yersinia pestis in human plasma: an approach for discovering bacterial genes involved in septicaemic plague. Microbiology, 2007. 153(Pt 9): p. 3112-24.

292. Klein, K.A., et al., A transposon site hybridization screen identifies galU and wecBC as important for survival of Yersinia pestis in murine macrophages. J Bacteriol, 2012. 194(3): p. 653-62.

293. Ponnusamy, D., et al., High-throughput signature-tagged mutagenic approach to identify novel virulence factors of Yersinia pestis CO92 in a mouse model of infection. Infect Immun, 2015.

294. Pereira-Leal, J.B. and M.C. Seabra, Evolution of the Rab family of small GTP-binding proteins. J Mol Biol, 2001. 313(4): p. 889-901.

295. Pereira-Leal, J.B. and M.C. Seabra, The mammalian Rab family of small GTPases: definition of family and subfamily sequence motifs suggests a mechanism for functional specificity in the Ras superfamily. J Mol Biol, 2000. 301(4): p. 1077-87.

296. Schwartz, S.L., et al., Rab GTPases at a glance. J Cell Sci, 2007. 120(Pt 22): p. 3905-10.

297. Diekmann, Y., et al., Thousands of rab GTPases for the cell biologist. PLoS Comput Biol, 2011. 7(10): p. e1002217.

298. Longatti, A. and S.A. Tooze, Recycling endosomes contribute to autophagosome formation. Autophagy, 2012. 8(11): p. 1682-3.

299. Alem, F., et al., Host response during Yersinia pestis infection of human bronchial epithelial cells involves negative regulation of autophagy and suggests a modulation of survival-related and cellular growth pathways. Front Microbiol, 2015. 6: p. 50.

300. Larson, C.L., et al., Coxiella burnetii effector protein subverts clathrin-mediated vesicular trafficking for pathogen vacuole biogenesis. Proc Natl Acad Sci U S A, 2013. 110(49): p. E4770-9.

301. Larson, C.L., et al., Coxiella burnetii Effector Proteins That Localize to the Parasitophorous Vacuole Membrane Promote Intracellular Replication. Infect Immun, 2015. 83(2): p. 661-70.

302. Newton, H.J., et al., A screen of Coxiella burnetii mutants reveals important roles for Dot/Icm effectors and host autophagy in vacuole biogenesis. PLoS Pathog, 2014. 10(7): p. e1004286.

303. Brock, S.C., J.R. Goldenring, and J.E. Crowe, Jr., Apical recycling systems regulate directional budding of respiratory syncytial virus from polarized epithelial cells. Proc Natl Acad Sci U S A, 2003. 100(25): p. 15143-8.

304. Hoffmann, C., et al., Functional analysis of novel Rab GTPases identified in the proteome of purified Legionella-containing vacuoles from macrophages. Cell Microbiol, 2013.

305. Mounier, J., et al., Shigella effector IpaB-induced cholesterol relocation disrupts the Golgi complex and recycling network to inhibit host cell secretion. Cell Host Microbe, 2012. 12(3): p. 381-9. 
306. Mellouk, N., et al., Shigella subverts the host recycling compartment to rupture its vacuole. Cell Host Microbe, 2014. 16(4): p. 517-30.

307. Smith, A.C., et al., Interaction of the Salmonella-containing vacuole with the endocytic recycling system. J Biol Chem, 2005. 280(26): p. 24634-41.

308. Guichard, A., et al., Cholera toxin disrupts barrier function by inhibiting exocyst-mediated trafficking of host proteins to intestinal cell junctions. Cell Host Microbe, 2013. 14(3): p. 294-305.

309. Galli, T., et al., Tetanus toxin-mediated cleavage of cellubrevin impairs exocytosis of transferrin receptor-containing vesicles in CHO cells. J Cell Biol, 1994. 125(5): p. 1015-24.

310. Wilcke, M., et al., Rab11 regulates the compartmentalization of early endosomes required for efficient transport from early endosomes to the trans-golgi network. J Cell Biol, 2000. 151(6): p. 1207-20.

311. Halaas, O., et al., Intracellular Mycobacterium avium intersect transferrin in the Rab11(+) recycling endocytic pathway and avoid lipocalin 2 trafficking to the lysosomal pathway. J Infect Dis, 2010. 201(5): p. 783-92.

312. Rowe, R.K., J.W. Suszko, and A. Pekosz, Roles for the recycling endosome, Rab8, and Rab11 in hantavirus release from epithelial cells. Virology, 2008. 382(2): p. 239-49.

313. Guichard, A., V. Nizet, and E. Bier, RAB11-mediated trafficking in host-pathogen interactions. Nat Rev Microbiol, 2014. 12(9): p. 624-34.

314. Ray, K., et al., Life on the inside: the intracellular lifestyle of cytosolic bacteria. Nat Rev Microbiol, 2009. 7(5): p. 333-40.

315. $\mathrm{Xu}, \mathrm{Y}$., et al., Toll-like receptor 4 is a sensor for autophagy associated with innate immunity. Immunity, 2007. 27(1): p. 135-44.

316. Pechous, R.D., et al., In vivo transcriptional profiling of Yersinia pestis reveals a novel bacterial mediator of pulmonary inflammation. MBio, 2015. 6(1): p. e02302-14.

317. Sivaraman, $\mathrm{V}$., et al., Yersinia pestis activates both IL-1beta and IL-1 receptor antagonist to modulate lung inflammation during pneumonic plague. PLoS Pathog, 2015. 11(3): p. e1004688.

318. Agar, S.L., et al., Characterization of the rat pneumonic plague model: infection kinetics following aerosolization of Yersinia pestis CO92. Microbes Infect, 2009. 11(2): p. 205-14.

319. Agar, S.L., et al., Characterization of a mouse model of plague after aerosolization of Yersinia pestis CO92. Microbiology, 2008. 154(Pt 7): p. 1939-48.

320. Comer, J.E., et al., Transcriptomic and innate immune responses to Yersinia pestis in the lymph node during bubonic plague. Infect Immun, 2010. 78(12): p. 5086-98.

321. Das, R., et al., Study of proinflammatory responses induced by Yersinia pestis in human monocytes using cDNA arrays. Genes Immun, 2007. 8(4): p. 308-19.

322. Du, Z., et al., Transcriptomic Response to Yersinia pestis: RIG-I Like Receptor Signaling Response Is Detrimental to the Host against Plague. J Genet Genomics, 2014. 41(7): p. 379-96.

323. Schoberle, T.J., et al., Uncovering an Important Role for YopJ in the Inhibition of Caspase1 in Activated Macrophages and Promoting Yersinia pseudotuberculosis Virulence. Infect Immun, 2016.

324. Zheng, Y., et al., YopJ-Induced Caspase-1 Activation in Yersinia-Infected Macrophages: Independent of Apoptosis, Linked to Necrosis, Dispensable for Innate Host Defense. PLoS One, 2012. 7(4): p. e36019.

325. Brodsky, I.E., et al., A Yersinia effector protein promotes virulence by preventing inflammasome recognition of the type III secretion system. Cell Host Microbe, 2010. 7(5): p. 376-87. 
326. Husebye, H., et al., The Rab11a GTPase controls Toll-like receptor 4-induced activation of interferon regulatory factor-3 on phagosomes. Immunity, 2010. 33(4): p. 583-96.

327. Czerkies, M., et al., An interplay between scavenger receptor $A$ and CD14 during activation of $J 774$ cells by high concentrations of LPS. Immunobiology, 2013. 218(10): p. 1217-26.

328. Nair-Gupta, P., et al., TLR signals induce phagosomal MHC-I delivery from the endosomal recycling compartment to allow cross-presentation. Cell, 2014. 158(3): p. 506-21.

329. Nair-Gupta, P. and J.M. Blander, An updated view of the intracellular mechanisms regulating cross-presentation. Front Immunol, 2013. 4: p. 401.

330. Weigert, R., et al., Rab22a regulates the recycling of membrane proteins internalized independently of clathrin. Mol Biol Cell, 2004. 15(8): p. 3758-70.

331. van ljzendoorn, S.C., Recycling endosomes. J Cell Sci, 2006. 119(Pt 9): p. 1679-81.

332. Neel, N.F., et al., Chemokine receptor internalization and intracellular trafficking. Cytokine Growth Factor Rev, 2005. 16(6): p. 637-58.

333. Haasken, S., et al., Macrophage scavenger receptor 1 (Msr1, SR-A) influences B cell autoimmunity by regulating soluble autoantigen concentration. J Immunol, 2013. 191(3): p. 1055-62.

334. D'Souza, R.S., et al., Rab4 orchestrates a small GTPase cascade for recruitment of adaptor proteins to early endosomes. Curr Biol, 2014. 24(11): p. 1187-98.

335. Johnson, D.E., et al., The position of lysosomes within the cell determines their luminal pH. J Cell Biol, 2016. 212(6): p. 677-92.

336. Fukui, G.M., et al., Studies on the relation of cultural conditions and virulence of Pasteurella pestis. J Bacteriol, 1957. 74(6): p. 714-7.

337. Higuchi, K., L.L. Kupferberg, and J.L. Smith, Studies on the nutrition and physiology of Pasteurella pestis. III. Effects of calcium ions on the growth of virulent and avirulent strains of Pasteurella pestis. J Bacteriol, 1959. 77(3): p. 317-21.

338. Barve, S.S. and S.C. Straley, IcrR, a low-Ca2(+)-response locus with dual Ca2(+)dependent functions in Yersinia pestis. J Bacteriol, 1990. 172(8): p. 4661-71.

339. Fowler, J.M., et al., Growth of calcium-blind mutants of Yersinia pestis at 37 degrees $C$ in permissive Ca2+-deficient environments. Microbiology, 2009. 155(Pt 8): p. 2509-21.

340. Perry, R.D., et al., DNA sequencing and analysis of the low-Ca2+-response plasmid pCD1 of Yersinia pestis KIM5. Infect Immun, 1998. 66(10): p. 4611-23.

341. Skrzypek, E. and S.C. Straley, Differential effects of deletions in IcrV on secretion of $V$ antigen, regulation of the low-Ca2+ response, and virulence of Yersinia pestis. J Bacteriol, 1995. 177(9): p. 2530-42.

342. Fader, C.M., et al., Induction of autophagy promotes fusion of multivesicular bodies with autophagic vacuoles in $k 562$ cells. Traffic, 2008. 9(2): p. 230-50.

343. Savina, A., et al., Rab11 promotes docking and fusion of multivesicular bodies in a calcium-dependent manner. Traffic, 2005. 6(2): p. 131-43.

344. Schwiesow, L., et al., The Yersinia type III secretion system master regulator LcrF. J Bacteriol, 2015. 


\section{APPENDIX}

Below are the acknowledgments and distribution permissions for each of the previouslypublished chapters (chapters I and III). Both published chapters are in PLOS (public library of science) open-access journals, which apply the Creative Commons Attribution (CC BY; creativecommons.org) license to all published materials. Under this license, anyone may reproduce, distribute, or reuses these articles as long as the author and original source are cited properly.

\section{Chapter II}

\section{Acknowledgements}

We thank M. Chelsea Lane and Harry Mobley for pGEN-luxCDABE plasmid, Herb Schweizer for the Tn7 system, Eric Weening and Virginia Miller for pUC18r6k-mini-Tn7(kanEW) plasmid, and Bill Goldman for the $\Delta$ pla strain. We would also like to thank the vivarium staff and the BIO-Imaging Core at the Center for Predictive Medicine for support during these experiments and Jonathan Warawa for sharing the PtolC template DNA, advice on in vivo imaging, and critical review of this manuscript. We also acknowledge helpful discussions throughout these studies with the faculty members of the Center for Predictive Medicine

\section{Chapter III}

\section{$\underline{\text { Acknowledgements }}$}

We thank James Galen (University of Maryland) and Harry Mobley (University of Michigan) for pGEN222 and pGEN-PEM7::DsREDand Dr. Shintaro Seto (Hamamatsu University School of Medicine) for pEGFP-Rab1B. The following reagent was obtained through BEI Resources, NIAID, $\mathrm{NIH}$ : Yersinia pestis, Strain KIM Derivative 19 (D19), NR-4681. We also thank Donghoon Chung for advice during these studies and Maria Gutierrez for helpful discussions. 


\section{DEPARTMENT OF MICROBIOLOGY AND IMMUNOLOGY CHAIRMAN'S CITATION}

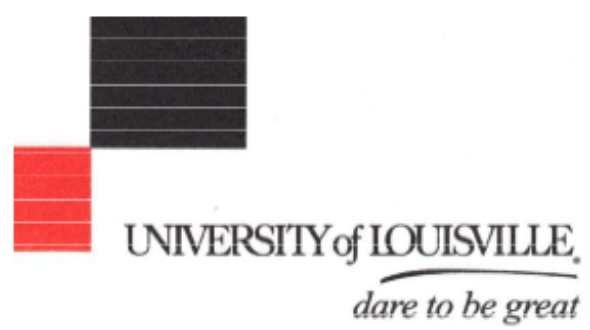

- DEPARTMEnT OF MICROBIOLOGY AND IMMUNOLOGY

School of Medicine University of Louisville Louisville, Kentucky 40292 Office: $502-852-5351$ Fax: $\quad 502-852-7531$

April 13, 2016

Mr. Michael Connor

Ph.D. Candidate

Department of Microbiology and Immunology

Dear Michael,

The Department of Microbiology and Immunology recognizes your dedication and hard work, which as communicated to me by your thesis advisor Dr. Matthew Lawrenz, has resulted in the publication of 2 first author papers in PLOS Pathogens and Frontiers in Microbiology. I am also aware of your pending first author paper and a second author publication in Plos One.

This level of productivity is indeed exceptional and sets a commendable example for all our students. I wish you continued success in your future endeavors.

Congratulations!

Sincerely,

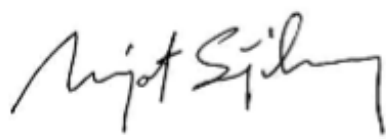

Nejat Egilmez, Ph.D.

Professor and Chairman

Department of Microbiology and Immunology 


\section{CURRICULUM VITAE}

Michael Graylin Connor

Work: Center for Predictive Medicine

Dept. of Microbiology and Immunology

505 S Hancock Street Rm 633

Louisville, KY 40202

Home: 734 Bermuda Lane Louisville, KY 40213

Born: February 10, 1988 Atlanta Georgia USA

Email: connormg@gmail.com

mgconn01@louisville.edu

\section{Education}

Ph.D Microbiology and Immunology, May 2016

University of Louisville, Louisville KY

Mentor: Matthew B. Lawrenz

Dissertation: "Identification of host factors required for Yersinia pestis macrophage Intracellular survival and their impact on vacuole maturation, acidification and trafficking"

M.S. Microbiology and Immunology, May 2012

University of Louisville, Louisville KY

Mentor: Matthew B. Lawrenz

B.S. Biology Pre-Health, May 2010

Lees-McRae College, Banner Elk NC

\section{Peer-reviewed Manuscripts ( ${ }^{*}$ denotes co-first author)}

1. Jason Nathaniel Payne*, Hitesh Kumar Waghwani*, Michael G Connor*, Sarah Tockstein, Harsh Moolani, William Robert Hamilton, Vivek Badwaik, Fenil Chavda, Matthew B Lawrenz, Rajalingam Dakshinamurthy. (2016) Novel Synthesis of Kanamycin Conjugated Gold Nanoparticles with Potent Antibacterial Activity. In press. Front. Microbiol. Antimicrobials, Resistance and Chemotherapy. Manuscript ID: 177773.

1. Connor MG, Pulsifer AR, Price CT, Abu Kwaik Y, Lawrenz MB (2015) Yersinia pestis Requires Host Rab1b for Survival in Macrophages. PLoS Pathog 11(10): e1005241. doi:10.1371/journal.ppat.1005241

2. Sun Y, Connor MG, Pennington JM, Lawrenz MB (2012) Development of Bioluminescent Bioreporters for In Vitro and In Vivo Tracking of Yersinia pestis. PLoS ONE 7(10): e47123. doi:10.1371/journal.pone.0047123 


\section{Oral presentations and invited lectures}

1. 2015-07-24 $2^{\text {nd }}$ Midwest Membrane Trafficking and Signaling Symposium, Louisville, KY. "Y. pestis Exploits Host Rab GTPases for Intracellular Survival"

2. 2015-07-28 Guest Research Presentation, Dr. Virginia Miller, University of North Carolina, Chapel Hill, NC.

"Genome-wide RNAi High-throughput Screen Identifies Host Trafficking as Critical for $Y$. pestis Intracellular Survival"

3. 2015-04-09 Microbiology \& Immunology Seminar Series. University of Louisville, Louisville, KY.

"RNAi High-throughput Screen Identifies Host Endocytic Trafficking as Critical for $Y$. pestis Intracellular Survival"

4. 2015-03-25 Bioinformatics Seminar Series. University of Louisville, Louisville, KY.

"Identifying How Y. pestis exploits the Host Macrophage"

5. 2014-10-31 Graduate School Research Symposium Series. University of Louisville, Louisville, KY.

"Understanding How Plague Manipulates the Host Macrophage"

6. 2013-10-31 Microbiology \& Immunology Seminar Series. University of Louisville, Louisville, KY.

"Exploiting the Host. Using a RNAi High-throughput Screen to Identify Host Factors Exploited by Y. pestis for Macrophage Survival"

7. 2012 Center for Predictive Medicine Retreat. University of Louisville, Louisville, KY.

"Unlocking Yersinia pestis Intracellular Survival"

\section{Posters and other scientific contributions (** selected for talk)}

1. 2015 Midwest Microbial Pathogenesis Conference, Indianapolis, IN.

"Yersinia pestis exploits the host endosome recycling pathway for intracellular survival"; Michael G. Connor, Amanda R. Pulsifer, Eric Rouchka, Donghoon Chung, Matthew B. Lawrenz.

2. ${ }^{* * 2015}$ 2nd Midwest Membrane Trafficking and Signaling Symposium, Louisville, KY.

"Y. pestis Exploits Host Rab GTPases for Intracellular Survival"; Connor M. G, Pulsifer A. $R$, and M.B. Lawrenz.

3. 2014 Gordon Research Conference, Toxins and Microbial Pathogenesis, Waterville Valley, $\mathrm{NH}$.

"RNAi High-Throughput Screen Identifies Novel Host Factors Exploited By Y. pestis For Intracellular Survival"; Michael G. Connor, Donghoon Chung, Matthew B. Lawrenz.

4. 2014 Gordon Research Conference, Toxins and Microbial Pathogenesis, Waterville Valley, $\mathrm{NH}$.

"Host Rab1B Is Required for Yersinia pestis Intracellular Survival"; Michael G. Connor, Amanda R. Pulsifer, Jarrod M. Pennington and Matthew B. Lawrenz.

5. 2014 Midwest Membrane Trafficking and Signaling Symposium, Louisville, KY.

"Host Rab1B Is Required for Yersinia pestis Intracellular Survival"; Michael G. Connor, Amanda R. Pulsifer, Jarrod M. Pennington and Matthew B. Lawrenz.

6. $201311^{\text {th }}$ International Conference on Yersinia, Suzhou, China. 
"Development of a High Throughput Assay to Identify Host Factors Required for Yersinia pestis Survival in Macrophages"; Michael G. Connor, Yanwen Sun, Donghoon Chung, and Matthew B. Lawrenz.

7. 2013 Midwest Microbial Pathogenesis Conference, Columbus, $\mathrm{OH}$.

"Unlocking Yersinia pestis intracellular survival from the host's perspective using an RNAi high throughput screen"; Michael G. Connor, Yanwen Sun, Donghoon Chung, and Matthew B. Lawrenz.

8. 2013 Research Louisville, Louisville, KY.

"Unlocking Yersinia pestis intracellular survival from the host's perspective using an RNAi high throughput screen"; Michael G. Connor, Yanwen Sun, Donghoon Chung, and Matthew B. Lawrenz (Finalist for Best Graduate Student Poster).

9. 2012 Research Louisville, Louisville, KY.

"Developing a bioluminescent high throughput siRNA screen for Yersinia pestis"; Michael G. Connor, Yanwen Sun, Jarrod M. Pennington, Donghoon Chung, and Matthew B. Lawrenz.

10. 2009 SEPEEG, Dahlonega, GA.

"Measure Correlation Between Levels of Genetic Variation and Infestation by the Hemlock Woolly Adelgid in Stands of Carolina Hemlock"; Michael G. Connor, Jennifer C. Hurd and Stephanie Stewart.

11. 2009 REU symposium, Athens, GA.

"Natronolimnobius 'aegyptiacus': A Novel Poly-extremophilic Archaeon Isolated from the Alkaline, Hypersaline Lakes of Wadi An Natrun, Egypt”; Michael G. Connor, Karen J.

Bowers, Noha M. Mesbah, Felipe Sarmiento and Juergen Wiegel.

\section{Fellowships}

2011-2013 University of Louisville IPIBS Graduate Fellowship

\section{Awards}

2016 Department of Microbiology and Immunology Chairman's Citation

2015 Graduate School Travel Award: Midwest Microbial Pathogenesis Conference (MMPC)

2014 University of Louisville Grant Writing Academy

2014-2016 University of Louisville SIGS Tuition Match Award

2013 Graduate School Travel Award: Midwest Microbial Pathogenesis Conference (MMPC)

2013 Research Louisville Top Poster Finalist: "Unlocking Yersinia pestis Intracellular Survival from the Host's Perspective Using a RNAi High Throughput Screen"

\section{Service and memberships}

2015 Kentucky Science Fair Judge

2015 Louisville Regional Science Fair Judge

2015 duPont Manual Science Fair Judge

2014 - Present Animal House Shelter Volunteer

2011-Present American Society for Microbiology (ASM)

2014-Present The American Society for Cell Biology (ASCB) 


\section{Employment}

\section{Ph.D Candidate}

Microbiology \& Immunology Ph.D Thesis Research

Dr. Lawrenz Lab, Center for Predictive Medicine; Louisville, KY

Title: Identification of host factors exploited by Yersinia pestis for survival in the macrophage.

Goal: Develop and complete a genome-wide RNAi high-throughput screen to identify host factors exploited by Yersinia pestis for survival within the macrophage.

2011-Present

\section{Ph.D Rotation Student}

Microbiology \& Immunology Ph.D Rotation

Dr. Mitchell Lab, Institute for Cellular Therapeutics; Louisville, KY

Task: Learn flow cytometry, primary cell culture, and Dendritic cell differentiation procedures.

2012

\section{Ph.D Rotation Student}

Microbiology \& Immunology Ph.D Rotation

Dr. Lawrenz Lab, Center for Predictive Medicine; Louisville, KY

Task: Develop a high-throughput RAW264.7 macrophage $Y$. pestis infection assay. 2011

\section{Laboratory Technologist I}

Products and Molecular Genetics Laboratory Technologist I

Bryce Burkhart, LabCorp; RTP, NC

Task: Produce oligomers and diagnostic testing reagents for commercial molecular genetics testing in a GLP compliant laboratory.

2010-2011

\section{Researcher}

Lees-McRae College: NC Biotech Grant Researcher

Dr. Chrissy Spencer, Lees-McRae College; Banner Elk, NC

Title: Measure Correlation Between Levels of Genetic Variation and Infestation by the Hemlock Woolly Adelgid in Stands of Carolina Hemlock.

Goal: Identify heterozygosity of Carolina Hemlock tree stands in relationship to Hemlock Woolly Adelgid infestation.

2009-2010

\section{Summer Researcher}

REU Researcher

Dr. Juergen Wiegel, University of Georgia; Athens, GA

Title: Characterization of a novel polyextremophile, Natronolimnobius 'aegyptiacus'.

Goal: Characterize the growth range of the novel polyextremophile Natronolimnobius 'aegyptiacus'.

2009 